\title{
POSTERIOR PROBABILITY JUDGEMENTS: DISTINGUISHING NUMERICAL OUTPUTS FROM THEIR UNDERLYING REASONING PROCESSES
}

\section{GAËLLE VILLEJOUBERT}

A thesis submitted in partial fulfilment of the requirements of the University of Hertfordshire for the degree of Doctor of Philosophy

The programme of research was carried out in the Department of Psychology, Faculty of Health and Human Sciences, University of Hertfordshire.

September 2003 
À ma mère, Avec tendresse et gratitude 


\section{Table of Contents}

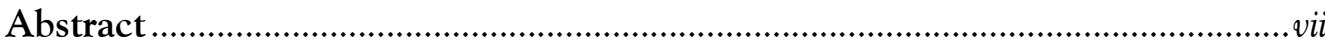

List of Figures ..................................................................................................... viii

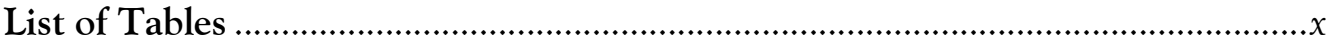

Symbols and abbreviations ............................................................................... xiii

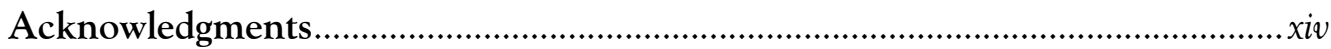

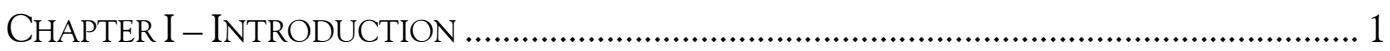

Epistemological perspective ..................................................................................... 3

Theoretical considerations for the process-focused study of posterior probability

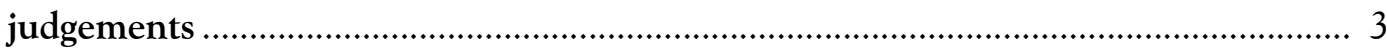

Normative and computational accounts of posterior probability judgements .... 5

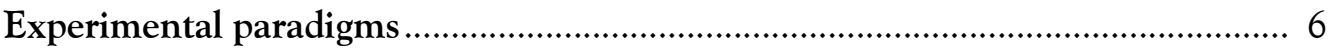

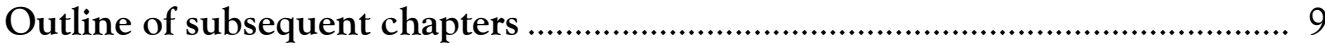

CHAPTER II - PhilosophicAl, MATHEMATICAL AND PSyCHOlOGICAL CONCEPTS OF

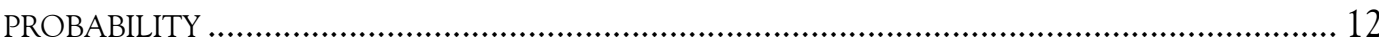

Probability as a mathematical concept................................................................. 13

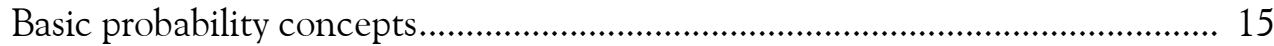

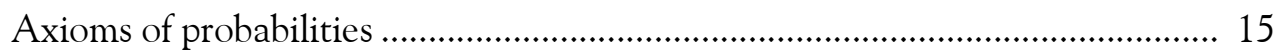

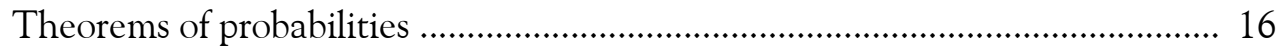

Probability as a philosophical concept ................................................................. 17

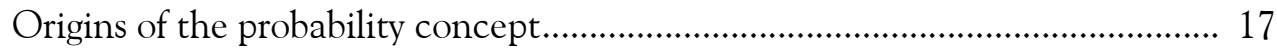

Probability in Antiquity and the Middle Ages ................................................. 17

Emergence of the mathematical probability concept .............................................. 18

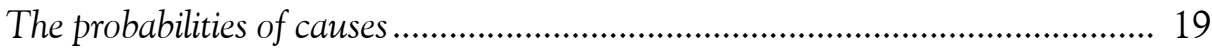

The Classical interpretation of probability …................................................... 20

Origins of the Classical interpretation ............................................................. 20

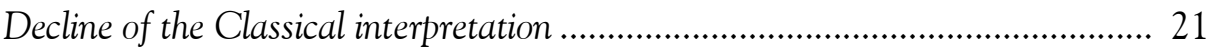

Modern conceptions of probability ………...................................................... 22

Frequentist conception of probability .......................................................... 22

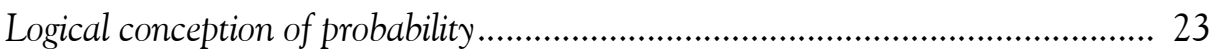

Subjective theory of probability ...................................................................... 24

Propensity theory of probability ...................................................................... 25

Concept(s) of probability within Psychology …................................................. 26 
Theoretical taxonomies of psychological probabilities ....................................... 26

Probability in posterior probability judgements................................................. 31

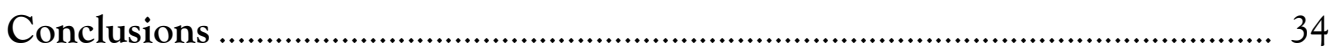

CHAPTER III - REVIEW OF COMPUTATIONAL AND ALGORITHMIC ACCOUNTS OF

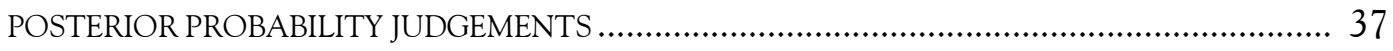

Concise historical context to the study of posterior probability judgements .... 37

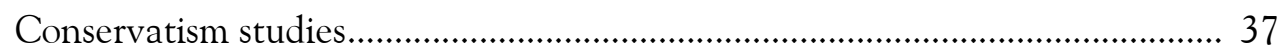

Heuristics and biases in judgements under uncertainty ................................. 39

The Social-judgement paradigm ........................................................................ 40

Presentation of the paradigm.................................................................................. 40

Computational accounts of judgment outcomes............................................... 42

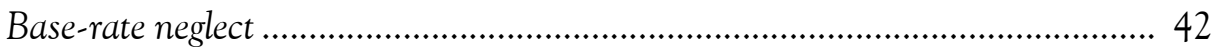

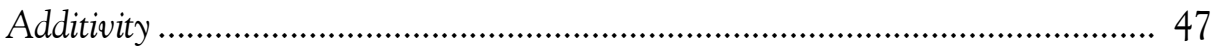

Algorithmic accounts of underlying reasoning processes ................................... 49

The representativeness heuristic....................................................................... 49

Problem representation and cognitive strategies .............................................. 50

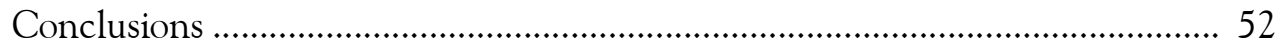

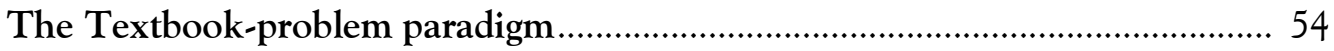

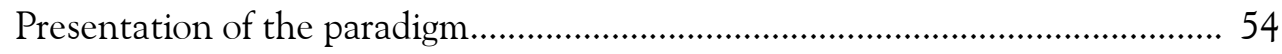

Computational accounts of judgment outcomes.............................................. 54

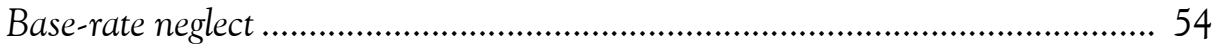

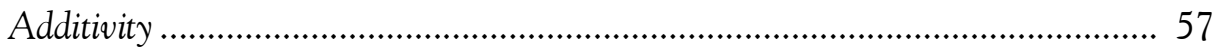

Algorithmic accounts of underlying reasoning processes ................................. 58

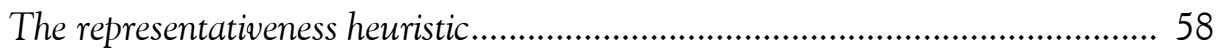

The causality account .................................................................................... 58

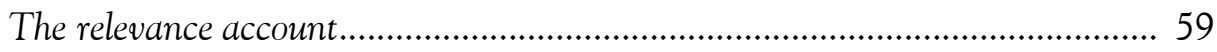

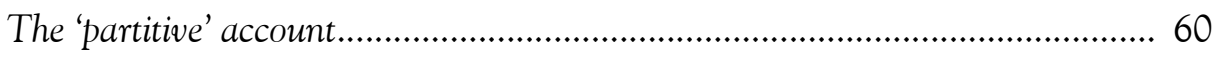

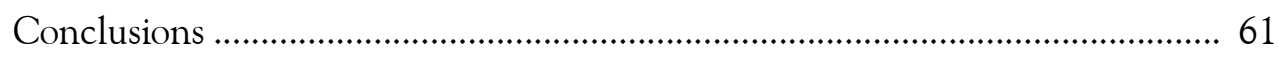

The Set-based paradigm ...................................................................................... 62

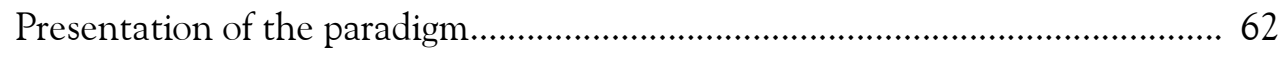

Computational accounts of judgment outcomes................................................. 63

Natural frequency formats lead to Bayesian judgements................................... 63

The role of natural frequencies challenged ...........................................................64

Can we tell that individuals are Bayesian reasoners? ....................................... 67 
Algorithmic account of underlying reasoning processes................................... 73

The evolutionary hypothesis ............................................................................. 73

The Mental Model Theory of probabilistic reasoning........................................ 74

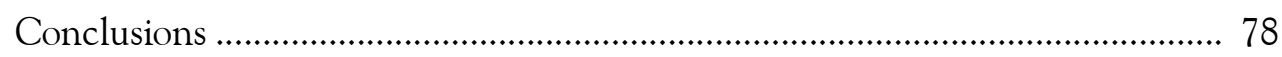

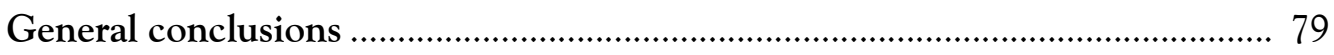

Synthesis of available computational accounts of the processes underlying

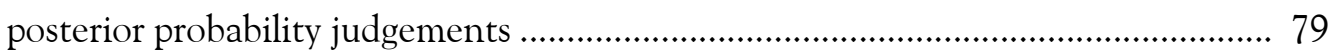

Synthesis of available algorithmic accounts of the determinants of posterior

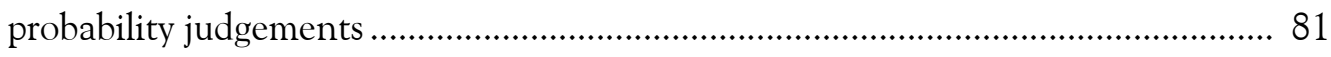

CHAPTER IV - ON THE INVERSE FALLACY AND THE SOCIAL-JUDGEMENT PARADIGM ...... 83

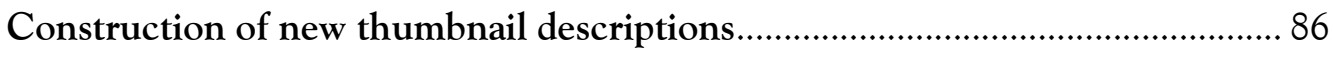

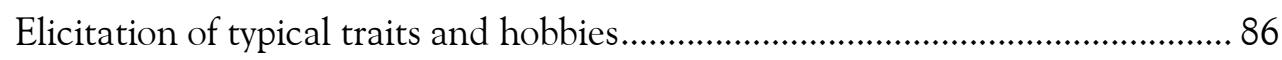

Construction and assessment of the descriptions' typicality ............................... 86

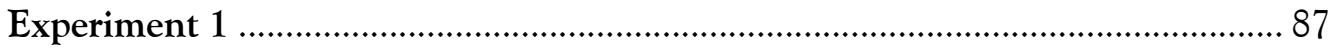

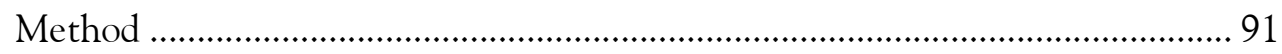

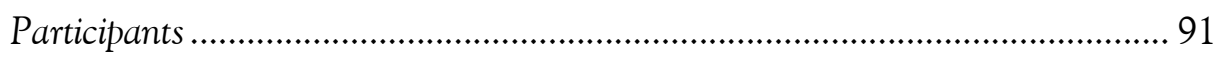

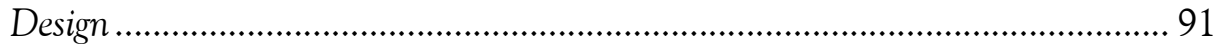

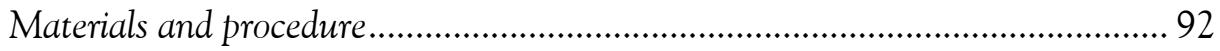

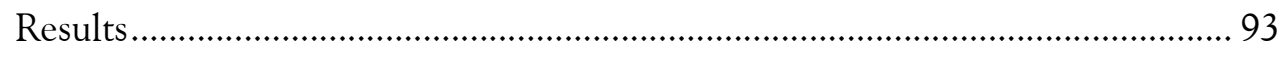

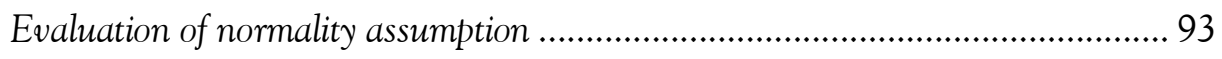

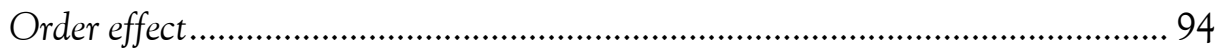

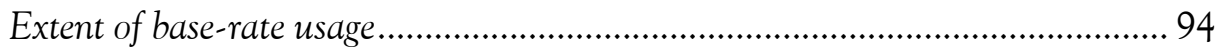

Analysis of judgements against Bayesian benchmarks........................................ 96

Inverse fallacy and posterior probability judgements ......................................... 99

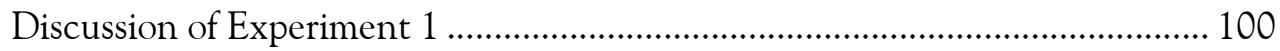

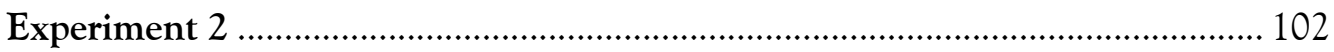

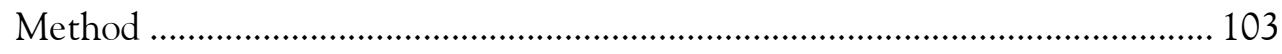

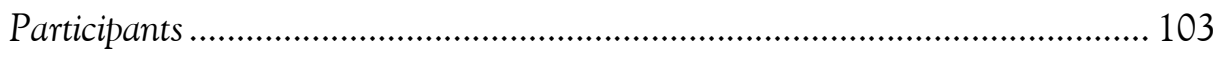

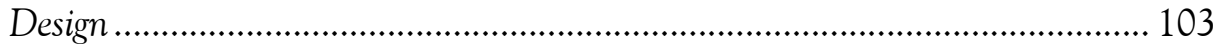

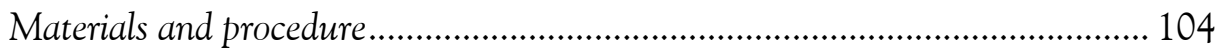

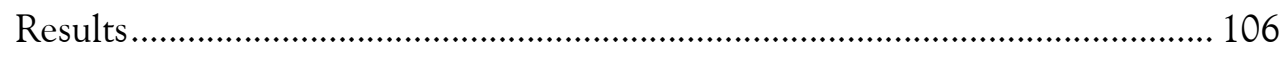

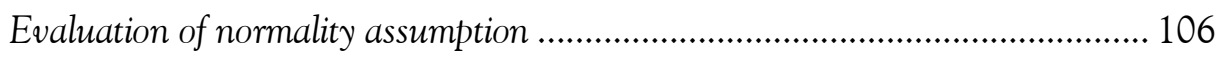

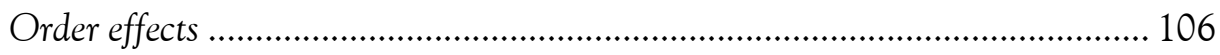

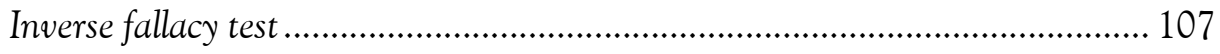




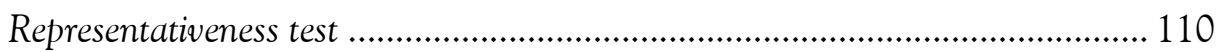

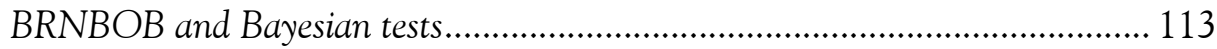

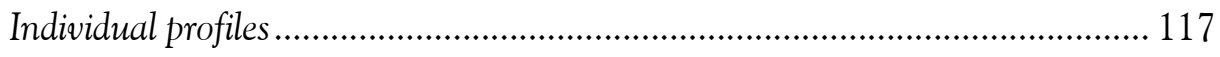

Deviations from the additivity principle ........................................................... 121

Discussion of Experiment 2 ............................................................................... 125

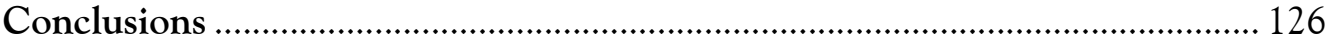

CHAPTER V - ON THE INVERSE FALLACY AND THE TEXTBOOK-PROBLEM PARADIGM ... 134

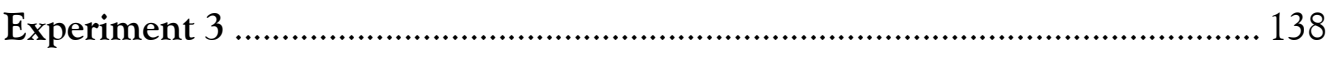

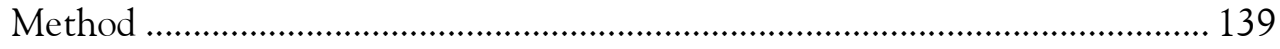

Participants ..................................................................................................... 139

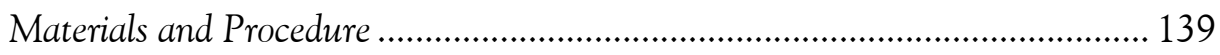

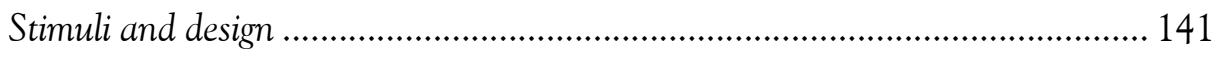

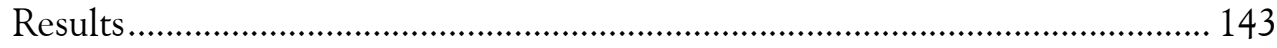

Classification of estimates..................................................................................... 143

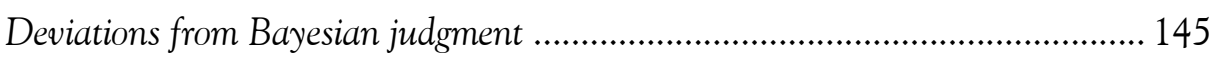

Deviations from the additivity principle ............................................................. 152

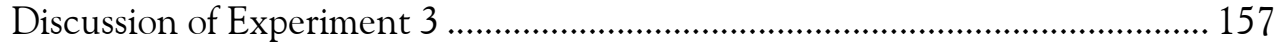

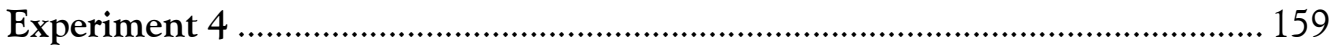

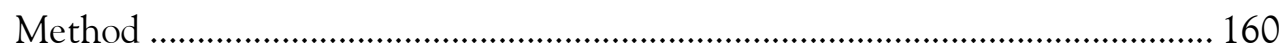

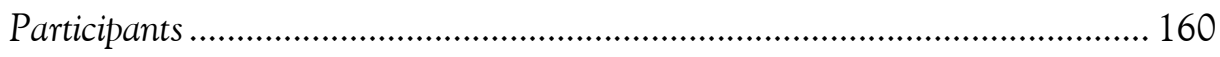

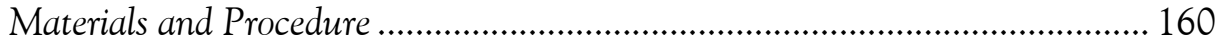

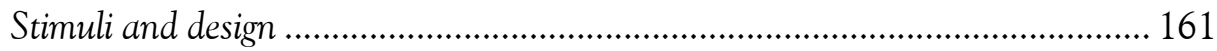

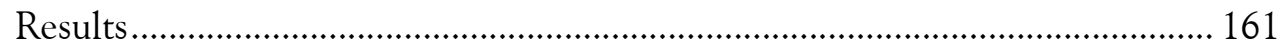

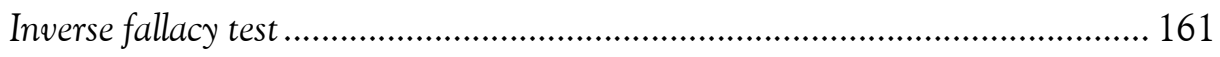

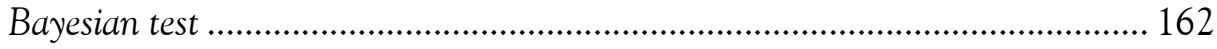

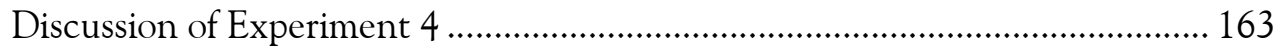

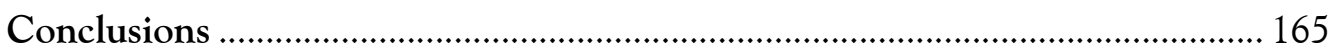

CHAPTER VI - ON BAYESIAN REASONING AND THE SET-BASED JUDGEMENT PARADIGM

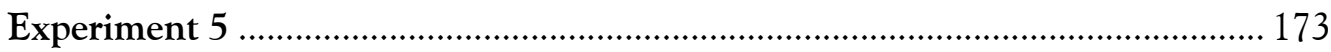

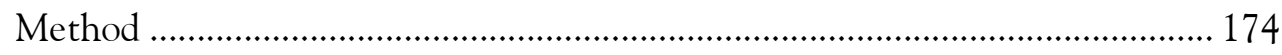

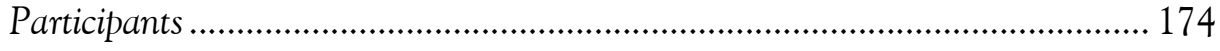

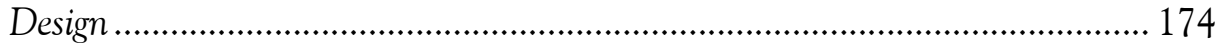

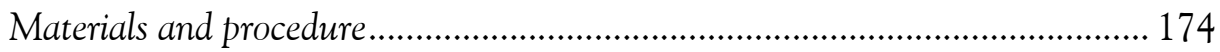




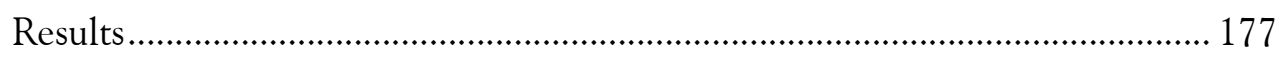

Judgement task ........................................................................................ 177

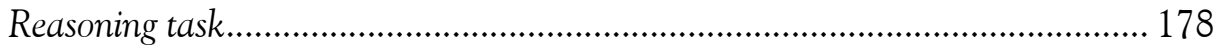

Probability interpretation task..................................................................... 180

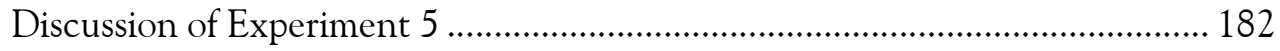

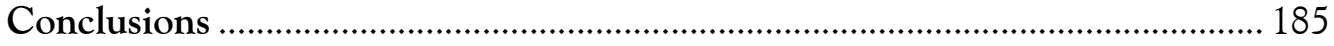

Chapter VII - SAmple Space RePReSeNTATION THEORY............................................. 187

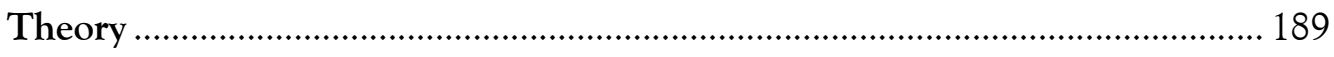

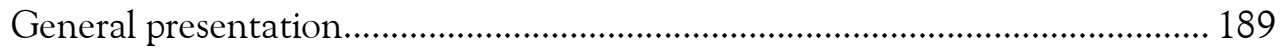

Initial sample space representation ..................................................................... 191

Type and intensions of task statements .......................................................... 191

Representation of elementary statements........................................................ 193

Representation of set statements .................................................................... 193

Representation of probability statements .......................................................... 194

Overview of initial representation stage .......................................................... 196

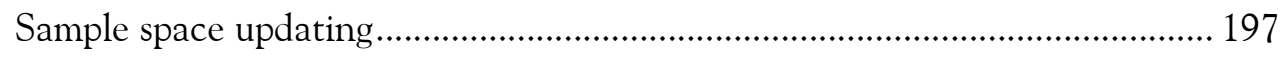

Updating elementary representations .............................................................. 197

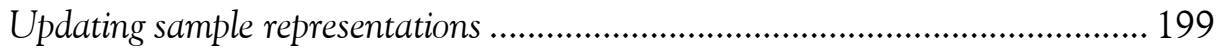

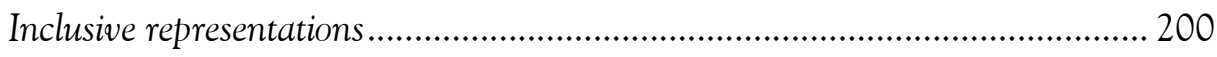

Multiple sample representations................................................................... 201

Overview of updating representation stage........................................................ 203

Final representations and judgement.............................................................. 205

Judgement methods and elementary goal statements ........................................ 206

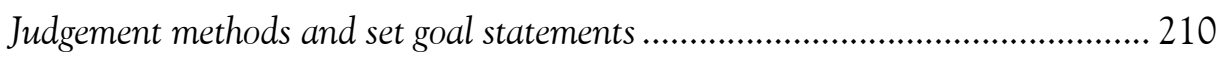

Overview of judgement stage .......................................................................... 214

Implications of SSR theory for the existing literature on posterior probability

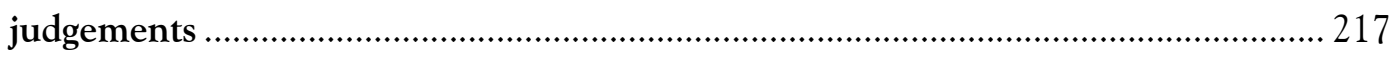

On the causality account (Tversky \& Kahneman, 1980) ................................. 217

The inverse fallacy and the taxicab problem ..................................................... 217

Accounting for the 'causal effect'.................................................................... 221

On the relevance account (Bar-Hillel, 1980) ………………………………..... 223

On the partitive account (Macchi, 1995, 2000) ................................................... 225

On the Mental Model theory of naïve probability judgements (Johnson-Laird et

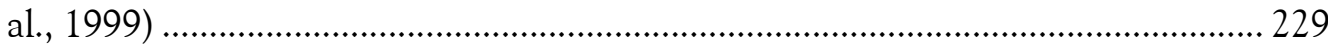




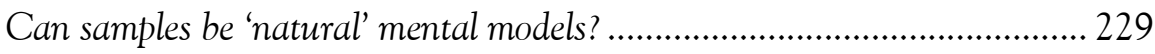

SSR theory vs. MMT applied to probabilistic reasoning................................ 231

Accounting for empirical findings of the thesis with SSR theory ..................... 238

Inverse fallacy and complementary goal statements .......................................... 238

Bayesian outcomes, natural frequencies and chance formats ......................... 241

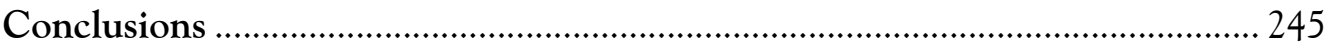

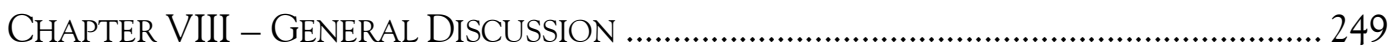

Computational analysis: Can one tell what judgement methods underlie

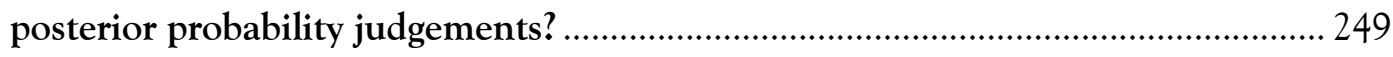

On the Social-judgement paradigm ………......................................................... 250

On the Textbook-problem paradigm ………..................................................... 253

On the Sample-based judgement paradigm......................................................... 256

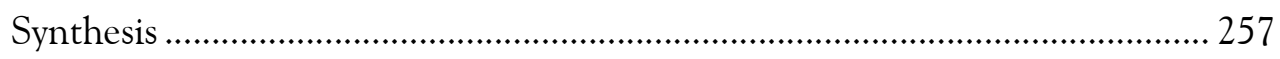

Algorithmic analysis: Can we tell how different posterior probability judgement

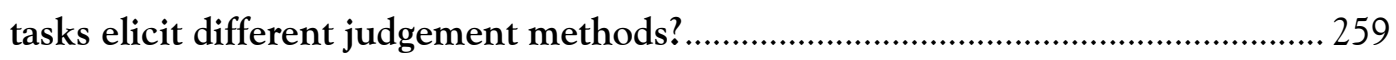

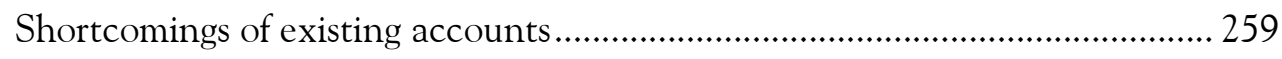

Sample Space Representation theory................................................................ 261

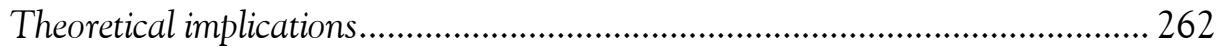

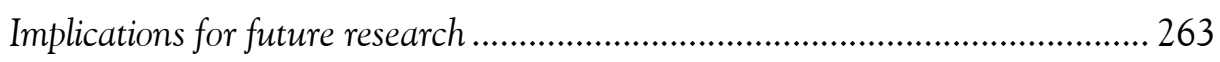

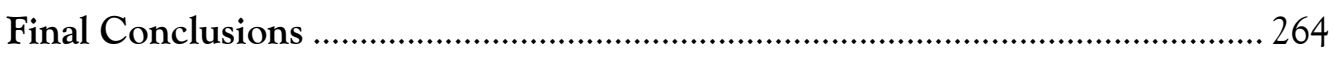

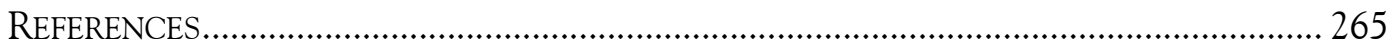

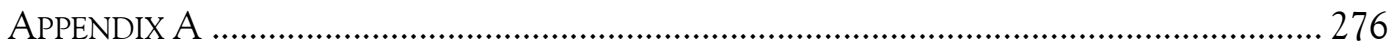

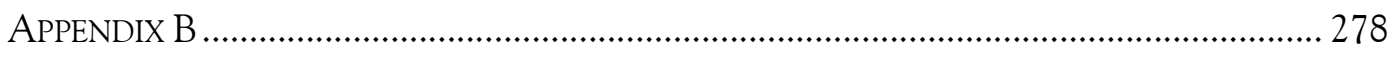




\begin{abstract}
$\underline{\text { Abstract }}$
This thesis aims to further our understanding of reasoning processes that underlie posterior probability judgements, i.e., judgements of the probability that a hypothesis is true, given new relevant information. Previous research has been output-focused and compared actual judgement outputs with normative Bayesian values. The thesis research has developed a new framework of analysis, the process-focused approach. This framework, paralleling work by Marr (1982), defines a new research agenda that entails i) the identification of what judgement methods underlie observed outputs (computational analysis), and ii) the explanation of how individuals' processing elicits the application of a particular judgement method (algorithmic analysis). The empirical work encompasses the three main posterior probability judgment paradigms and is aimed at distinguishing between competing current computational accounts. Within the Social-judgement paradigm, although aggregated posterior judgements are found to be identical to inverse likelihood judgements, further results show that no existing computational account reliably predicts individual behaviour. Within the Textbookproblem paradigm, outputs are commonly underpinned by the application of the inverse fallacy. This method is also found to be less prevalent when only one of two complementary posterior probability judgements is elicited. In the Set-based paradigm, most judgement outputs are Bayesian, but Bayesian outputs do not necessarily entail successful Bayesian reasoning. This computational analysis of judgements together with the appraisal of existing algorithmic accounts led to the development of the Sample Space Representation theory. SSR theory provides a thorough yet general account (i) explaining how Textbook problems and Set-based tasks are represented, and (ii) characterising the cognitive processes underpinning the application of the judgement methods previously identified.
\end{abstract}




\section{List of Figures}

Figure II-1 - Basic concepts of Set theory

Figure II-2 - Venn diagram representation of the conditional probability of $\mathrm{H}$

given $D$

Figure III-1 - Normative relation between low-engineer and high-engineer posterior probabilities for $k^{2}=5.44$ (adapted from Kahneman \& Tversky, 1973).

Figure III-2 - Gigerenzer et al.'s (1988) schema of human probability revision ...... 51

Figure III-3 - Venn diagrams of fully inclusive vs. multiple sample information structure in the mammography problem

Figure IV-1 - Display of the pilot questionnaire presented to check the diagnosticity of the five thumbnail descriptions

Figure IV-2 - Mean typicality ratings as a function of the description presented, with standard errors 90

Figure IV-3 - Plot of the median posterior probability judgements observed in the high base-rate condition against those observed in the low base-rate condition

Figure IV-4 - Plot of the mean posterior probability judgements observed against the mean Bayesian values computed for the high and low base-rate condition 97

Figure IV-5 - Plots of the mean posterior probability judgements observed against the inverse likelihood judgements for the high and low base-rate condition

Figure IV-6 - Layout of the questionnaire used in Experiment 2 105

Figure IV-7 - Individual scatterplots of posterior probability judgements as a function of inverse likelihood judgements for each description and each target hypothesis..... 108

Figure IV-8 - Individual scatterplots of posterior probability judgements as a function of representativeness judgements for each description and each target hypothesis.... 111

Figure IV-9 - Individual scatterplots of posterior probability judgements as a function of BRNBOB values for each of description and each target hypothesis used.............. 114

Figure IV-10 - Individual scatterplots of posterior probability judgements as a function of Bayesian values for each description and each target hypothesis 116

Figure IV-11 - Hasse diagram of the 16 mutually comparable strategy profiles .... 120

Figure IV-12 - Individual scatterplot of the posterior probability judgements for the 'designer' category as a function of the posterior probability judgements for the 'accountant' category 
Figure IV-13 - Overall scatterplot of the posterior probability judgements for the 'designer' category as a function of the posterior probability judgements for the 'accountant' category 124

Figure V-1 - Template of the questionnaire layout used in Experiment 3 ........... 140

Figure V-2 - Frequency of different number of judgements matching the value tested (Bayesian, Inverse) as a function of type of measure $[p(H \mid D), p(\sim H \mid D)]$ 145

Figure V-3 - Observed deviations of $p(H \mid D)$ and $p(\sim H \mid D)$ judgements from Bayesian values as a function of expected magnitudes and expected direction of deviation 150

Figure V-4 - Observed summed complementary posterior probability judgements as a function of expected magnitudes of deviations and expected direction of deviation from additivity

Figure V-5 - Hypothesized use of probability information, as a function of the questions asked (single vs. complementary)

Figure V-6 - Distribution of the number of judgements matching their corresponding inverse values in Experiments 3 and 4, respectively....

Figure V-7 - Distribution of the number of judgements matching their corresponding Bayesian values in Experiments 3 and 4, respectively 163

Figure VI-1 - Questionnaire layout used in Experiment 5. 176

Figure VII-1 - Flow diagrams comparing the processes involved in probability judgement and problem solving 190

Figure VII-2 - Flow diagram summarising the processes involved in the elaboration of the initial representation of a posterior probability judgement task. 196

Figure VII-3 - Flow diagram summarising the processes involved in the updating of the initial representation of a posterior probability judgement task 204

Figure VII-4 - Flow diagram summarising the processes underlying the final judgement output in posterior probability judgement tasks.....

Figure VII-5 - Fully explicit representations based on Euler circles of the statement "some artists are beekeepers" 


\section{List of Tables}

Table I-1 - Characteristic features of the three experimental paradigms for the study of one-shot posterior probability judgements

Table II-1 - Teigen's (1994) taxonomy of quasi-analytical and analytical rules that may be used as a basis for probability judgements

Table IV-1 - Stereotypical traits and hobbies/interests attributed to accountants and designers by 15 judges

Table IV-2 - Thumbnail descriptions constructed from a gathered sample of typical traits

Table IV-3 - Comparison of mean judgements observed in high and low base-rate conditions 96

Table IV-4 - Comparison of mean judgements observed in high and low base-rate conditions with their Bayesian counterparts

Table IV-5 - Simple contrasts of mean judgements observed in high and low base-rate conditions compared to inverse likelihood judgements, for each description 100

Table IV-6 - Parameter estimates for the inverse fallacy test 109

Table IV-7 - Parameter estimates for the representativeness heuristic test .... 112

Table IV-8 - Parameter estimates for the BRNBOB test................................. 115

Table IV-9 - Parameter estimates for the Bayesian test.................................... 117

Table IV-10 - Strategy profiles of the 16 participants...................................... 119

Table IV-11 - Median $\mathrm{p}(\mathrm{H} \mid \mathrm{D})$ and $\mathrm{p}(\sim \mathrm{H} \mid \mathrm{D})$ posterior probabilities judgements, for each description

Table IV-12 - Parameter estimates for the additivity test .....

Table V-1 - Proportion of Gloms and Fizos presenting each of twelve features, encountered creature's response, and corresponding inverse and Bayesian values

Table V-2 - Expected deviations of posterior probability judgements from Bayesian benchmarks as a function of the magnitude and direction of the deviation of inverse values from Bayesian values for both $p(H \mid D)$ and $p(\sim H \mid D)$ measures

Table V-3 - Expected deviations of the sum of complementary posterior probability judgements [i.e., $p(\mathrm{H} \mid \mathrm{D})+p(\sim \mathrm{H} \mid \mathrm{D})$ ] from additivity as a function of 
the magnitude and direction of the deviation of the sum of corresponding inverse values from 1

Table V-4 - Wilcoxon's matched-pairs signed-ranks test statistics for the comparison of the distribution Bayesian and Inverse number of matches, for each type of judgement elicited.

Table V-5 - Mean deviation scores from Bayesian prescriptions as a function of expected direction of deviation, expected magnitude of deviation and conditioning datum, for each of the two posterior probability measures collected ..................... 146

Table V-6 - Summary of the 2 (conditioning datum) $\times 2$ (expected direction of deviation) $\times 3$ (expected magnitude of deviation) doubly multivariate repeatedmeasure ANOVA with $p(H \mid D)$ and $p(\sim H \mid D)$ judgements as dependent measures ..

Table V-7 - Univariate repeated-measures ANOVAs for the $p(H \mid D)$ and the $p(\sim H \mid D)$ measures

Table V-8 - Planned marginal comparisons for the Direction of deviation $\times$ Magnitude of deviation interaction effect for the $p(H \mid D)$ and $p(\sim H \mid D)$ measures

Table V-9 - Mean additivity scores as a function of expected direction of deviation, expected magnitude of deviation and conditioning datum ......

Table V-10 - Univariate Analyses of Variance for the additivity scores ......

Table V-11 - Planned marginal comparisons for the Direction of deviation $\times$ Magnitude of deviation interaction effect for the additivity scores

Table VI-1 - Classification grid used to classify outputs ................................. 177

Table VI-2 - Judgement outputs observed..................................................... 177

Table VI-3 - Reasoning method choices observed............................................ 178

Table VI-4 - Cross-tabulation of judgement output and reasoning choice ..... 179

Table VI-5 - Matrix for evaluating the consistency of judgement outputs and reasoning choices

Table VI-6 - Frequencies of observation of each combination of probability type and information format

Table VI-7 - Frequencies of Bayesian reasoning choices observed as a function of the type and format of the single-event probability statement interpretation ........ 182

Table VII-1 - Overview of the propositions of Sample Space Representation theory 
Table VII-2 - Overview of the corollaries derived from the propositions of Sample Space Representation theory …………..................................................... 247 


\section{Symbols and abbreviations}

$\Leftrightarrow \quad$ mathematical equivalence

universal set; contains all possible elements within the particular application under consideration

$S=\{x \mid i(x)\} \quad$ denotes the intensional description of the set $S$, defined as consisting of all elements $x$ described by the intension $i$

$S=\{b l u e\} \quad$ alternative notation to denote the intensional description of the set $S$ consisting of all elements $x$ described by the intension $i(x)=$ " $x$ is blue."

$S=\left\{x_{1}, \ldots, x_{n}\right\}$ denotes the extensional description of the set $S$, defined as consisting of elements $x_{k}$ for $k=1$ to $n$

$x \in S \quad$ indicates that $x$ is an element of the set $S$

$x \notin S \quad$ indicates that $x$ is not an element of the set $S$

$S_{1} \cup S_{2} \quad$ denotes the union of sets $S_{1}$ and $S_{2}$

$S_{1} \cap S_{2} \quad$ denotes the intersection of sets $S_{1}$ and $S_{2}$

$S_{2} \subset S_{1} \quad$ denotes the subset relationship; reads " $S_{2}$ is a subset of $S_{1}$ " denotes the empty or null set denotes intension $i$ of element $x$; that is, the specific way in which a defining property of $x$ is characterised reads "there is an element $x$ such that $x$ is described by intension $i$ " reads "all $x$ are described by intension $i$ "

$\wedge$ logical connector 'and'; used to denote that elements are described by several intensions, such as in $i_{1}\left(x_{0}\right) \wedge i_{2}\left(x_{0}\right)$

$\vee$ logical connector 'or' used to denote that elements are described by an intension or another, but not both, such as in $i_{1}\left(x_{0}\right) \vee i_{2}\left(x_{0}\right)$ $\neg \quad$ explicit negation

$\sim \quad$ implicit negation, complementarity

$\therefore \quad$ therefore

$p(H \mid D) \quad$ denotes the posterior probability of event $H$ given the reduced sample space $D$. 


\section{$\underline{\text { Acknowledgments }}$}

My initial thanks are addressed to Prof Denis Hilton for having guided my first steps into the world of research, and for adding the anglo-world to my universe. I would also like to thank Dr. Fred ValleyTourangeau, for having given me the opportunity to do this thesis and for his manifest confidence in my competences. Even after his departure, the memory of his enthusiasm and his conviction often helped me to continue to go forward, in spite of my uncertainties. I also thank Dr. David Mandel. His insightfulness, his patience and his meticulous re-reading certainly played a great part in the publication of our article on the inverse fallacy in a journal such as Memory \& Cognition. I hope this thesis pays tribute to the standards of excellence he taught me. I would also like to express my deep gratitude towards Dr. Diana Kornbrot and Dr. Stefanie Schmeer for giving me their support, both academic and personal, in the final stretch of my doctoral studies. A great amount of credit goes to them for their meticulous second reading, their thoughtful and always respectful comments, and their support. In particular, it is thanks to these encouragements that I found the confidence I needed to develop my ideas on sample space representations. My sincere thanks also go to my two examiners, Prof Vittorio Girotto and Prof Ken Manktelow, for their meticulous reading of my work as well as for the quality of their comments and their compliments.

Apart from the formal circle of supervision, my cordial thanks also go to my colleagues and/or friends: Prof Peter Ayton, Dr. David Hardman, Dr. Clare Harries, Prof Nigel Harvey, Dr. Steffi Kurzenhaüser, Dr. John Maule, Dr. Alastair McClelland, Olga Mourouti, Anneli Nilsson, Dr. Mike Page, Prof Alan Pearman, Dr. Mel Powell, Dr. Daniel Read, Dr. Lily Read, for their support and their encouragements.

Furthermore, a warm thanks, from deep in my heart, to Ciarán O'Keeffe, for his support, his tenderness, his humour, his love, steady, and comforting, at my side: I am looking forward to spending the rest of my life with you.

Lastly, my warmest and most tender thanks goes to my mother, for her courage, her love, her confidence without limits, and for having made me understand the value of things, and especially that of Education: You are in my heart, today like yesterday, and for ever.

\section{2ok}

Mes premiers remerciements s'adressent à Prof. Denis Hilton, pour avoir guidé mes premiers pas dans le monde de la recherche, et pour avoir étendu mon univers au monde anglophone. Je tiens également à remercier Dr. Fred Vallée-Tourangeau, pour m'avoir donné la possibilité de faire cette thèse et pour sa confiance manifeste en mes compétences. Même après son départ, le souvenir de son enthousiasme et de sa conviction m'a souvent aidé à continuer à aller de l'avant, en dépit de mes incertitudes. Je remercie aussi Dr. David Mandel. Sa perspicacité, sa patience et sa relecture méticuleuse ont certainement joué un grand rôle dans la parution de notre article sur l'erreur d'inversement dans un journal tel que Memory $\mathcal{G}^{2}$ Cognition. J'espère que cette thèse rend hommage aux standards d'excellence qu'il m'a enseignés. Je tiens à exprimer ma profonde gratitude envers Dr. Diana Kornbrot et Dr. Stefanie Schmeer, pour avoir accepté de m'apporter leur soutien autant académique que moral dans la dernière ligne droite de mon parcours doctoral. Une grande part de crédit leur revient pour leur relecture minutieuse, leurs commentaires avisés mais toujours respectueux, et leurs encouragements. En particulier, ce sont ces encouragements qui m'ont donné la confiance qui me manquait pour développer mes idées sur la représentation des ensembles. Mes sincères remerciements s'adressent également à mes deux examinateurs, Prof. Vittorio Girotto et Prof. Ken Manktelow pour leur lecture minutieuse de mes travaux ainsi que pour la qualité de leurs commentaires et leurs compliments.

En dehors du cercle formel de supervision, mes chaleureux remerciements s'adressent également à mes collègues et/ou amis : Prof. Peter Ayton, Dr. David Hardman, Dr. Clare Harries, Prof. Nigel Harvey, Dr. Steffi Kurzenhaüser, Dr. John Maule, Dr. Alastair McClelland, Olga Mourouti, Anneli Nillsson, Dr. Mike Page, Prof. Alan Pearman, Dr. Mel Powell, Dr. Daniel Read, Dr. Lily Read, pour leur soutien et leur encouragements.

Aussi, un grand merci, du fond du cour, à Ciarán O'Keeffe, pour son soutien, sa tendresse, son amour, infaillible et réconfortant, à mes cotés : Je suis impatiente de passer le reste de ma vie avec toi.

Enfin, mes plus chaleureux et plus tendres remerciements s'adressent à ma mère, pour son courage, son amour, sa confiance sans limites, et pour m'avoir fait comprendre la valeur des choses, et surtout celle de l'Éducation: Tu es dans mon coeur, aujourd'hui comme hier, et pour toujours. 
... some errors and biases in judgment under uncertainty call for a dual analysis: a positive account that explains the choice of a particular erroneous response in terms of heuristics and a negative account that explains why the correct rule has not been learned.

(Kahneman \& Tversky, 1982/1982b, p. 507)

he aim of this thesis is to develop a 'process-focused' approach to the study of human behaviour in the realm of posterior probability judgements. Posterior probability judgements are involved when one needs to update the prior probability that a hypothesis is true in the light of relevant new information. They are involved in everyday judgements as well as numerous high-stakes situations such as the medical situation where a physician must update the prior probability that a patient has a particular disease in the light of the observation of a diagnostic symptom; or the court situation where jurors have to update the prior probability that a person is guilty in the light of new forensic evidence. From this practical perspective, a crucial issue is whether we can form accurate posterior probability judgements.

From a theoretical standpoint, posterior probability judgements are also critical within the fields of Cognitive and Social Psychology, respectively. In relation to cognition, posterior probability judgements are important for the study of probabilistic reasoning processes, or reasoning under uncertainty. For instance, posterior probability judgements can be involved in inductive reasoning where judges infer the probability of a new albeit uncertain conclusion (e.g., that a hypothesis is true), from the information made available to them. Likewise, in relation to social behaviour, posterior probability judgements are important with regards to the study of behavioural attribution and stereotypical judgements. For instance, a stereotypical judgement can be conceptualised as the assessment of the probability that an individual has a certain personality trait, given his social category membership. Hence, understanding how we form posterior probability judgements can in turn inform our understanding of human stereotyping.

When studying posterior probability judgements, psychologists are faced with two important questions. On the one hand, they may take the perspective that the general issue to be addressed is whether our posterior probability judgements are accurate or normative. From the realisation that human judgements generally depart from normative benchmarks, psychologists may thereafter engage in the development of an outcome-focused account of posterior probability judgements. Such an account entails the 
examination of the reasons behind the lack of accuracy of posterior probability judgements and aims to identify the external conditions or appropriate aiding tools that would improve the accuracy of those judgements. On the other hand, an alternative approach consists in developing a process-focused account of posterior probability judgements. Within this approach, the main research agenda no longer consists in achieving the elicitation of normative judgements. A process-focused research agenda will instead consist of (i) attaining a systematic description of judgement methods or judgement strategies employed (i.e., the method by which the outcome of judgement, whether or not normative, is produced), and (ii) developing a theoretical account of the processes underlying the application of the method or methods thus identified.

The term 'process-focused' is intended here to refer to a general epistemological approach, as defined in the aforementioned agenda. It is in contrast with the term 'process-based' approach, which would refer to the type of empirical data upon which a study is based (i.e., the tracing of the processes surrounding reasoning or judgement; see e.g., Abelson \& Levi, 1985). Similarly, the term 'outcome-focused' is intended to refer to the general epistemological approach aiming at further developing our knowledge of the conditions under which judgements' outcomes are normative. It is to be differentiated from the term 'outcome-based' which also refers to the type of empirical data upon which a study is based (i.e., the observable outcomes of the judgement). Both process-focused and outcome-focused approaches may or may not entail process-based and outcome-based examinations of behaviours.

The main thrust of this thesis will be to initiate and develop a process-focused study of posterior probability judgements. This thesis does not aim to provide a totally exhaustive identification of the judgement methods underlying the outcomes of posterior probability judgements or a definitive theoretical explanation for the processes underlying the application of those methods. Nevertheless, the reviews of concepts presented in the next two chapters, the experiments reported in the subsequent three chapters, and the new theoretical account proposed in the penultimate chapter, are aimed to add substantially to our knowledge of the psychological processes underlying posterior probability judgements. 


\section{EPISTEMOLOGICAL PERSPECTIVE}

The choice of a process-focused approach to study posterior probability judgements derives from my personal epistemological standpoint, namely the view that the primary basis for the study of human behaviour needs to be descriptive rather than normative. Hence, to choose to follow a process-focused approach is to attempt to identify and understand the processes underlying judgement strategies as opposed to taking part in the debate about the rationality of observed behaviours. By taking this position, I do not mean to argue that normative approaches should be dismissed altogether. As the previous examples of posterior probability judgements in high stake situations illustrates, the extent to which our judgements are normative is a crucial issue. Yet our understanding of human behaviour needs to extend beyond the knowledge of the conditions under which such judgements are normative or rational. One way to achieve this objective is by increasing our knowledge of the mechanisms that lead these behaviours to either deviate from or be in line with normative benchmarks.

The call for the development of our understanding of the cognitive processes underlying observed judgement outcomes has already been voiced (e.g., Slovic \& Lichtenstein, 1971). Yet most current and past research on posterior probability judgements has been underpinned by an outcome-focused approach and therefore aimed at identifying the conditions under which posterior probability judgements are normative, seemingly equating numerical outputs with the behaviours underlying these outputs (see chap. III).

\section{THEORETICAL CONSIDERATIONS FOR THE PROCESS-FOCUSED STUDY OF POSTERIOR PROBABILITY JUDGEMENTS}

The research agenda set by the process-focused study of behaviours draws on Marr's (1982) distinction between computational and algorithmic theoretical considerations. Marr (1982) proposed that 'information processing systems' can be conceptualised from three different levels of analysis: the computational, algorithmic and implementational levels. Marr (1982) applied his analysis to the conceptualisation of human vision. This approach, however, can be extended to any information-processing system which produces a response, following the processing of a task's premises and goal. In the present thesis, for instance, such a 'system' can be defined as the judge who provides a judgement following the processing of a posterior probability judgement task. 
At the computational level, the conceptualisation of the information-processing system defines what is computed, encompassing both a statement of the system competences and the description of the system's output. With regards to the study of posterior probability judgements, the objective of such a level of analysis is taken to be twofold. Firstly, since the goal of the judge is to produce a probability judgement, a computational analysis would require that the object of the information-processing task (i.e., the concept of probability) be unambiguously defined, alongside the characterisation of the system's capability. Thus, a necessary albeit preliminary task for the computational analysis of a system producing posterior probability judgement should consists of a substantive review of the different philosophical and mathematical interpretations of the concept of probability as well as the characterisation of what may constitute an individual's psychological understanding of this concept. The second objective of a computational analysis is to describe what the system does; in other words, at the computational level, the study of posterior probability judgements involves the development of a valid description of individuals' judgements. This is indeed the primary objective of the process-focused approach developed within this thesis. Different levels of descriptions can be used. For instance, one could simply say that individuals making a posterior probability judgement 'produce a number.' The aim of the computational analysis of posterior probability judgements developed in this thesis, however, will be to provide a valid description of the judgement methods or strategies applied by individuals.

At the algorithmic level, the conceptualisation of a system consists in defining how the procedures defined at the computational level are achieved. According to Marr (1982), this level of analysis entails the characterisation of the steps being carried out to produce the output, including the specification of the representation upon which these steps depend. Thus, with regards to posterior probability judgements, this level of analysis calls for the characterization of the processes and representations underlying the application of the judgement methods that were identified at the computational level of analysis.

Finally, at the implementation level, the conceptualisation consists in specifying how the processes identified at the algorithmic level are physically implemented. Such a level of analysis of posterior probability judgements would entail identifying the brain mechanisms allowing the algorithmic conceptualisation to be realised. The process- 
focused approach outlined in this thesis, however, is limited to the development of the computational and algorithmic analyses of posterior probability judgements.

\section{NORMATIVE AND COMPUTATIONAL ACCOUNTS OF POSTERIOR PROBABILITY JUDGEMENTS}

Normative judgements of posterior probabilities should follow Bayes's theorem. Suppose that $H$ is a hypothesis such as " $x$ is an engineer" and $D$ is a relevant or diagnostic piece of information such as " $x$ enjoys mathematical puzzles." The probability that $H$ is true, prior to the gain of information $D$, is referred to as the prior probability of $H$, and is denoted by $p(H)$. If such a probability is based on frequencies, then $p(H)$ can also be referred to as the base rate of $H$. For instance, if the probability that $x$ is an engineer is based on the relative frequency of engineers in the population under consideration, then $p$ (engineer) corresponds to the base rate of engineers in this population.

To update the prior probability that a hypothesis $H$ is true, given the gain of the information $D$, is to evaluate $p(H \mid D)$, read as 'the posterior probability of $H$ given $D$ '. To do so, Bayes's theorem prescribes that $p(H)$, and $p(\sim H)$, read as 'the probability of non- $H^{\prime}$, should be combined with $p(D \mid H)$ and $p(D \mid \sim H)$; where $p(\sim H)$ represents the prior probability that $H$ is false, $p(D \mid H)$ represents the likelihood of observing the data $D$ when $H$ is known to be true, and $p(D \mid \sim H)$ represents the likelihood of observing $D$ when $\mathrm{H}$ is false. The plural term likelihoods will be used throughout the thesis to denote both conditional probabilities $p(D \mid H)$ and $p(D \mid \sim H)$ at once. Precisely, Bayes's theorem specifies that the posterior probability that $H$ is true in the light of the data $D$ should be evaluated on the basis of the following combination of prior probabilities and likelihoods:

$$
p(H \mid D)=\frac{p(D \mid H) \cdot p(H)}{p(D \mid H) \cdot p(H)+p(D \mid \sim H) \cdot p(\sim H)}
$$

The psychological study of posterior probability judgements was initiated by Edwards and his colleagues in the 60s (e.g., Phillips \& Edwards, 1966). This line of research revealed that the direction of people's sequential revision of prior probabilities in the light of new data was in line with Bayesian prescriptions. Yet it was found that the magnitude of their revision was consistently less than that prescribed by Bayes's theorem. This effect was labelled conservatism. In contrast, in the 70s, Kahneman and Tversky (e.g., Kahneman \& Tversky, 1973) initiated the study of single-shot posterior probability 
judgements where individuals were simply asked to provide a unique posterior probability judgement on the basis of relevant information. The investigation of singleshot posterior probability judgements soon revealed that these judgements often drastically departed from Bayesian prescriptions.

Most subsequent studies in this domain of research thereafter focused on the identification of both the external causes for those departures from normative standards and the potential remedies to improve judgmental accuracy. These studies heavily relied on analyses of outcomes of judgements (see Koehler, 1996, for a review). Outcomefocused accounts culminated with the realisation that the format used to present information has a major effect on people's judgements. In particular, it was found that certain formats of information presentation such as frequencies arising from natural sampling (Gigerenzer \& Hoffrage, 1995) could lead to judgements whose numerical value perfectly matched that prescribed by Bayes's theorem. As a result, the absence of Bayesian performance observed in earlier studies is now customarily attributed to the format of the probabilistic information presented in these former problems.

In this thesis, a process-focused approach is applied to the examination of single-shot posterior probability judgements. As previously mentioned, the objectives of the processfocused approach are somewhat different from those of the outcome-focused approach just outlined. A process-focused approach calls for (i) a computational analysis of the judgements; that is, the development of a precise description of the judgement methods underlying the outcomes observed within the different paradigms used for studying posterior probability judgements, and (ii) an algorithmic analysis; that is, the development of a theoretical account of the processes and representations underlying the application of the judgement methods identified by the computational analysis.

\section{EXPERIMENTAL PARADIGMS}

There are three experimental paradigms within which "single-shot" posterior probability judgements are now studied: Bar-Hillel (1983) distinguished the Socialjudgement paradigm from the Textbook-problem paradigm. Recently, a new paradigm was introduced, which I will refer to as the "Set-based paradigm". Although most research within this paradigm focuses on the role of frequency-based information on posterior probability judgements, I chose the label "Set-based" as opposed to "Frequency-based" because-as shown later in the thesis-crucial aspects of this 
paradigm relate to the use of set-based statements, whether or not the elements within these sets can be assimilated to frequencies of occurrence. This thesis systematically tackles the process-focused research agenda identified in the previous section, first by reviewing both existing computational and algorithmic accounts and then by bringing together experiments within each of these three paradigms. The distinction between these paradigms is not always made explicit within the literature (but see Bar-Hillel, 1983; Macchi, 1995, note 1, p. 190). This is perhaps because, from an outcome-focused perspective, the implications of findings observed in each paradigm greatly overlap, as judgements are conceptualised in terms of their departure from Bayesian standards in general, and in terms of their lack of integration of the base-rate information, the socalled "base-rate fallacy" (e.g., Bar-Hillel, 1980; Koehler, 1996; Tversky \& Kahneman, 1980).

The systematic, process-focused, review of these paradigms, however, will reveal that Social-judgement, Textbook-problem and Set-based paradigms not only differ with regards to the type of tasks used to elicit posterior probability judgements, but also with regards to the computational and algorithmic accounts invoked to explain the results observed. Table I-1 summarises the main differences between the three paradigms by illustrating the information given in typical tasks and examples of the main computational and algorithmic accounts proposed. These features are presented and discussed in detail from a process-focused perspective in chapter III. The following section presents an outline of the different chapters of the thesis. 


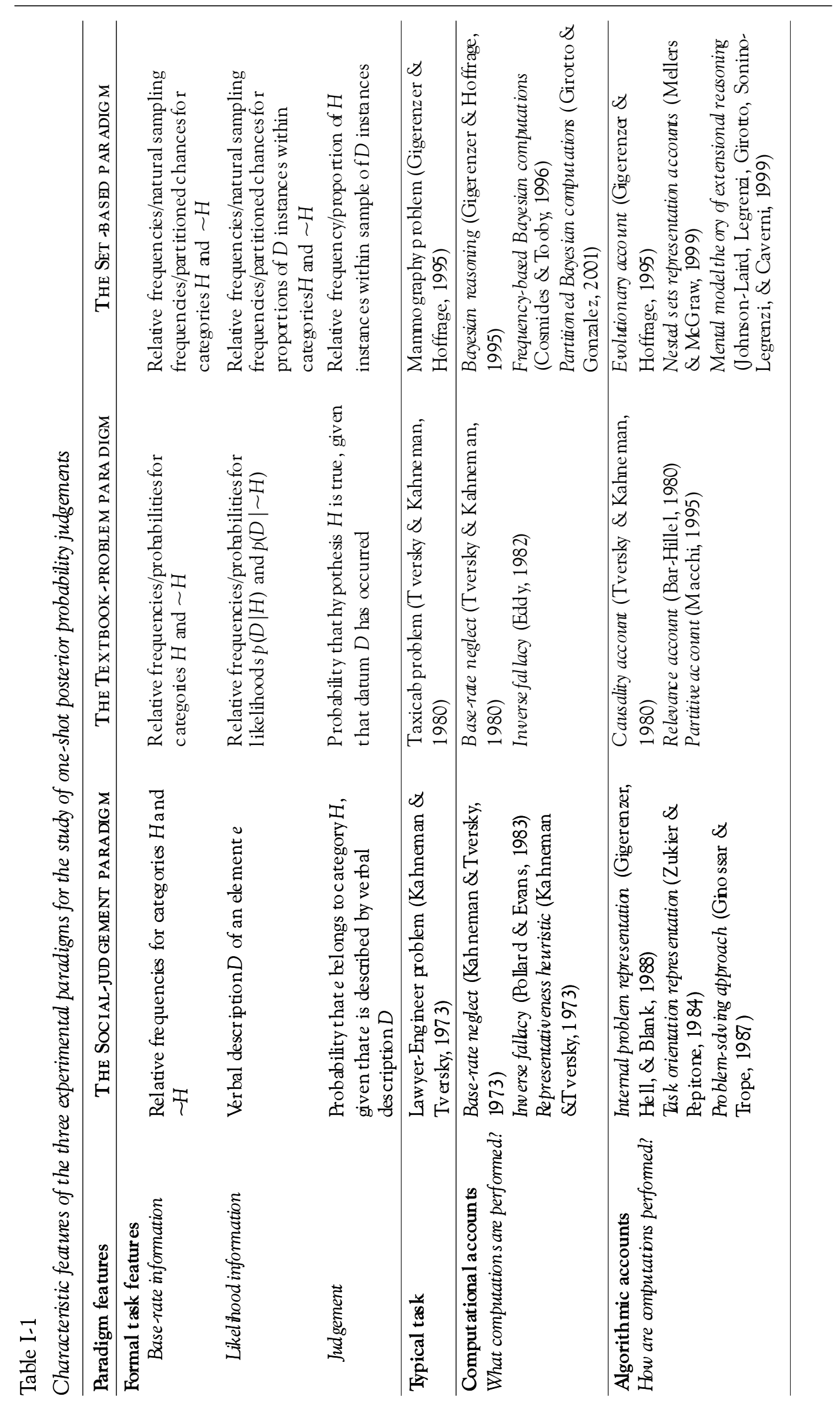


OUTLINE OF SUBSEQUENT CHAPTERS

\section{Chapter II}

Philosophical, mathematical and psychological concepts of probability

As previously suggested, the computational analysis of posterior probability judgements should be grounded in a thorough understanding of the concept of probability itself, in order to achieve a psychological grasp of posterior probability judgements. Therefore, the next chapter (chapter II) introduces the different mathematical and philosophical concepts of probability. This introduction, in turn, informs the review of the concept of probability as it occurs within psychological studies of posterior probability judgements. Such a review reveals that although some authors have worked at clarifying the concept of probability within the psychological framework, it appears that there is little empirical knowledge related to individuals' concept(s) or even representations of probabilities.

\section{Chapter III}

Review of computational and algorithmic accounts of posterior probability judgements

The process-focused approach to the study of posterior probability judgements firstly entails the development of a rigorous computational account of people's judgements (i.e., identify what individuals do when making posterior probability judgement). Secondly, this approach calls for the development of an algorithmic account which characterised how the procedures defined at the computational level are achieved. In chapter III, with such guiding principles in mind, existing accounts of single-shot posterior probability judgements are reviewed within each of the three experimental paradigms mentioned above and their potential contribution to the development of a process-focused study of these judgements is assessed. The issues identified in turn set about the empirical work developed in the subsequent chapters of the thesis.

\section{Chapter IV}

On the inverse fallacy and the Social-Judgement paradigm

Chapter IV presents a study comprising two experiments belonging to the Socialjudgement paradigm. The main proposition tested is that judgements of posterior probabilities observed within this paradigm are in fact best described by the inverse fallacy, rather than by the representativeness heuristic or the neglect of base-rate 
information hypothesis. A secondary objective is to investigate whether complementary posterior probability judgements are additive.

\section{Chapter V}

On the inverse fallacy and the Textbook-problem paradigm

Chapter $\mathrm{V}$ presents an empirical study comprising two experiments investigating posterior probability judgements within the Textbook-problem paradigm. The primary objective of this study is to distinguish between base-rate fallacy and inverse fallacy computational accounts while investigating the origin of the deviations from Bayesian prescriptions and the additivity principle. In the second part of this chapter, the determinants of the inverse fallacy are investigated further.

\section{Chapter VI}

\section{On Bayesian reasoning and the Set-based paradigm}

In chapter VI, the relationship between underlying reasoning processes and numerical outputs within the Set-based paradigm is investigated. In studies originating from this paradigm, most participants' judgements are "normative;" that is, judgment outputs match Bayesian outputs. As a result, the focus of posterior probability judgement studies has recently shifted from the discussion of the factors underlying nonnormative judgements to that of the factors that enable normative judgements to be elicited. Subsequent to the distinction between Bayesian algorithms and Bayesian reasoning, the relationship between these two computational accounts is investigated in a last experimental study.

\section{Chapter VII}

Sample Space Representation theory

In chapter VII, I introduce a new algorithmic theory, called Sample Space Representation (SSR) theory, which I developed over the course of my Ph.D. research. The theory's purpose is to explain how single-shot judgements of posterior probabilities may be mediated by the particular sample representation elicited by the task in both Textbook and Set-based problems. SSR theory is based on the assumption that individuals' probability judgements are guided by their mental representation of the statements in probability judgement tasks. This representation is assumed to be dynamic and updated as the judge processes further information. Specifically, the content of the sample space representation is assumed to be determined by the type (i.e., elementary vs. 
set) and the intension (i.e., the specific description) of the quantitative statements of the task. The propositions of the theory unequivocally define the exact modalities by which different sample space representations are elicited by different tasks. These propositions also precisely characterise the processes by which judgements are elicited. In the final section of this chapter, the implications of Sample Space Representation theory for existing findings are discussed. It is shown that the theory provides a compelling explanation for a wide range of new and old empirical results observed within both the Textbook-problem paradigm and the Set-based paradigm.

\section{Chapter VIII}

\section{General discussion}

In the final chapter, the contribution of the thesis to knowledge of computational and algorithmic processes underlying posterior probability judgements is appraised. The chief empirical findings are summarised and methodological and theoretical implications are discussed. The discussion revolves around two main points of consideration; that is, the extent to which the work presented in the thesis can inform (i) what judgement methods underlie posterior probability judgements, and (ii) how different tasks elicit different judgement methods. The chapter concludes with the suggestion of directions for future work.

\section{sk}


We must find some connection between this abstract entity which satisfies certain mathematical stipulations and the pragmatic content, the real meaning, of the important statements of scientific and social intercourse which contain the word "probability."

(Kyburg \& Smokler, 1964, pp. 3-4)

W hat is a probability? At first sight, defining the concept of probability might seem to be a simple task given its interpretation in its common everyday usage. For example, we would assume that everyone can intuitively understand that 'the probability of rain' is 'the likelihood that rain will occur'. Yet it is not as straightforward as it seems. In French, for example, the word 'likelihood' is absent from the common language, and hence, the semantic differentiation between probability and likelihood simply does not exist. And even beyond mere linguistic differences, the vast amount of literature that only focuses on the meaning of probability suggests that this concept can hardly be defined in such a simple, intuitive manner. An important milestone for the achievement of a scholar understanding of how posterior probability judgements are made-the aim of the present thesis-therefore becomes the examination of the concept of probability itself.

In the present chapter, probability as a modern mathematical concept is distinguished from its different current and past philosophical interpretations. Thus, following the presentation of mathematical probability as well as its axioms and its theorems, the different interpretations of the probability concept, developed since Antiquity up until the present day, are reviewed. This historical and philosophical review brings to light the long-lasting subjective-objective duality of the concept. The relatively recent theoretical distinction between subjective and objective interpretations of probabilities_-based on degrees of belief and the natural sampling of frequencies, respectively-is thus put into perspective and the diverse existing criteria of "correctness" for the measurement of probabilities are identified. Finally, this enables an enlightened appraisal of the concept of probability and informs the recent psychological debates vis-à-vis the human interpretation of probabilities, within the Judgement and Decision-Making research domain. 


\section{PROBABILITY AS A MATHEMATICAL CONCEPT}

Although the probability calculus is nearly unchallenged today, it was only formalised at the beginning of the twentieth century by Kolmogorov (e.g., see Howson \& Urbach, 1993). The probability axioms, which form the calculus of probability, are independent of the meaning of probability and thus apply to any interpretational framework: "subjective" and "objective" probabilities of events, for instance, are all governed by the same four mathematical axioms. Kolmogorov originally presented his axioms using a Measure theoretic approach, which is an extension of the Set theory, a branch of Mathematics whose purpose is the formalisation of the properties of collections of objects called 'sets' (Colman, 2001).

In particular, Set theory terminology can offer a useful tool for a "neutral" definition of probability that is free of reference to its different philosophical interpretations. This approach will serve as a basis for theoretical developments in Chapter VIII. Brief definitions of the basic concepts of Set theory (sets, set operations, set relationships) are presented in the top section of Figure II.1. These definitions are adapted from Budnick (1988), Mosteller, Rourke, and Thomas (1970), Book (1977), and Vaught (1995). 


\section{Basic Set theoretic concepts}

Set. A set refers to a group of objects called elements, which possess a certain attribute or attributes required for membership in the set. A set can be denoted by enumeration (i.e., by listing all its elements) or by description (i.e., by describing the attribute(s) of its elements).

The notations " $\in S$ " and " $\notin S$ " are used to indicate that $x$ is, or is not, an element of the set $S$, respectively. The number of elements contained in the set $S$ is denoted by $n(S)$.

Example. A standard 52-card deck can be denoted by the enumeration $A=\{|\boldsymbol{*}, \ldots|,, \ldots$,

$\mid \boldsymbol{\vee}, \ldots, K \boldsymbol{K}\}$ or the description $A=\{x \mid$ " $x$ is a playing card" $\}$, which reads " $A$ is the set consisting

of all elements $x$ 'such that' (')' symbol) $x$ is a playing card.

To refer to the extension of a set is to refer to the members composing a given set,

independently of their description. To refer to the intension of a set is to refer to the specific description used to define set membership.

Example. Suppose an urn contains black and white balls. The sets $A=\{x \mid$ " $x$ is a white ball" $\}$ and

$B=\{x \mid$ " $x$ is not a black ball" $\}$ have the same extension but different intensions.

Set relationships. $\mathscr{S}$ denotes the universal set, which contains all possible elements within a particular application under consideration. The complement of a set $S$ is the set $\sim S$ (also noted S), which consists of all elements in the universal set $\mathscr{S}$ that are not members of $S$.

Example. if $\mathscr{S}=\{x \mid x$ is a woman $\}$, and $S=\{x \mid x$ is a 25 -year old woman $\}$, then the complement of $S$,

viz., $\sim S$ is defined by the set of women who are not 25 years old.

The symbol $\varnothing$ denotes the set consisting of no elements, called the empty or null set. $S_{2}$ is defined as a subset of $S_{1}$ if and only if every element of $S_{2}$ is also an element of $S_{1}$. The subset relationship is denoted as $S_{2} \subset S_{1}$ and reads "S2 is a subset of $S_{1}$ " or "S2 is included in $S_{1} . "$

Set operations. Two sets, $S_{1}$ and $S_{2}$ are equal if and only if every element of $S_{1}$ is an element of $S_{2}$ and every element of $S_{2}$ is an element of $S_{1}$, i.e., $S_{1}=S_{2}$ iff $S_{1} \subset S_{2}$ and $S_{2} \subset S_{1}$. The union of two sets $S_{1}$ and $S_{2}$, denoted by $S_{1} \cup S_{2}$, is a set which consists of all elements contained in either $S_{1}$ or $S_{2}$. The intersection of two sets, $S_{1}$ and $S_{2}$, denoted by $S_{1} \cap S_{2}$, is a set which consists of elements both contained in $\mathrm{S}$ I and in S2. Set relationships and set operations are illustrated by Venn diagrams (i) and (ii), respectively.

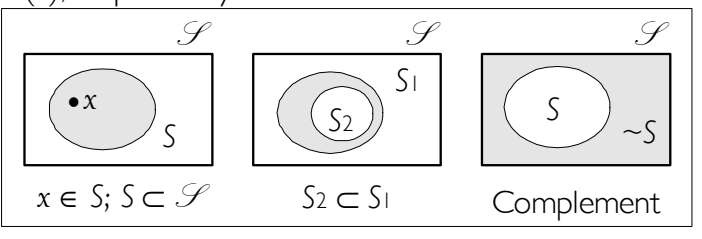

(i) Venn diagram representation of set relationship

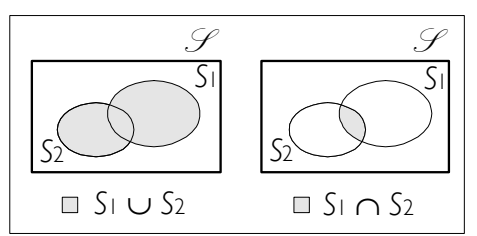

(ii) Venn diagrams for set operations

\section{Basic Set theoretic probability concepts}

Probability concepts. An experiment is a random process resulting in one of a number of possible outcomes. The sample space of an experiment is the universal set $\mathscr{S}$, which includes all possible outcomes of the experiment. Each repetition of an experiment is a trial. Each trial has one and only one outcome, called a simple outcome or element of $\mathscr{S}$.

Example. The random selection of a ball from an urn containing a proportion of black and white balls is an experiment in which each draw represents a trial and the result of the draw, a single outcome.

The sample space of the experiment is defined by $\mathscr{S}=\{$ white ball, black ball $\}$.

Sets of experimental outcomes are called events. A simple event is a collection of identical simple outcomes. A compound event is a collection of several types of simple outcomes grouped on the basis of a common attribute. To say that "the event $E$ has occurred" is to say that a trial has resulted in the outcome $e$ where $E=\{x \mid x$ has the attribute $e\}$.

Example. $B=\{b \mid b$ is a black ball $\}$ is a simple event of the afore mentioned experiment. $E=\{e \mid e$ is an odd number of dots facing up $\}$ is a compound event of a dice throw experiment.

Two events are said to be mutually exclusive if the occurrence of the former proscribes the occurrence of the later; that is, if $E_{1} \cap E_{2}=\varnothing$. Two events are said to be collectively exhaustive if their union accounts for all possible outcomes of an experiment; that is if $E_{1} \cup E_{2}=\mathscr{S}$.

Figure II-1 - Basic concepts of Set theory. 


\section{Basic probability concepts}

Set theory can prove very useful in defining mathematical probabilities, their axioms and theorems. The bottom section of Figure II-1 presents basic probability concepts in a Set theoretic formalisation. The next section on "Probability as a philosophical concept" will reveal that up until this day, scholars are still discussing how the elements of $\mathscr{S}$ ought to be defined. Yet it is not necessary to settle on one interpretation or another to define the axioms and theorems of probability. Although the set theoretic terminology used has strong Frequentist connotations, the concepts of experiment, outcome and event, for instance, need not be interpreted in such a restricted context. The concept of experiment may also encompass the test of a hypothesis $H$ or a proposition $P$, and elements of the sample space $\mathscr{S}$ can be conceptualised as outcomes, degrees of belief in the occurrence of outcomes, or chances for the occurrence of outcomes, etc. It is because elements of sets are not restricted to the results of random processes that the Set theoretic formalisation remains independent of philosophical interpretations of probability. This also explains why axioms and theorems of probabilities-presented in the next sub-section-are accepted by authors from different philosophical perspectives.

\section{Axioms ${ }^{1}$ of probabilities}

\section{Axiom 1}

To each set $H$ of the sample space $\mathscr{S}$, a nonnegative number $p(H)$ is attached. This number $p(H)$ is called "the probability of the event $H$ ".

\section{Axiom 2}

The probability of the entire sample space $\mathscr{S}$ is 1 .

\section{Axiom 3 - Addition law of probability}

The probability of the union of $H_{1}$ and $H_{2}$ is defined by

$$
p\left(H_{1} \cup H_{2}\right)=p\left(H_{1}\right)+p\left(H_{2}\right)-p\left(H_{1} \cap H_{2}\right)
$$

\section{Axiom 4-Multiplication law of probability}

The probability of the intersection of $\mathrm{H}_{1}$ and $\mathrm{H}_{2}$ is defined by

$$
p\left(H_{1} \cap H_{2}\right)=p\left(H_{2}\right) \cdot p\left(H_{1} \mid H_{2}\right)
$$

\footnotetext{
${ }^{1}$ Adapted from Kolmogorov (1933, as cited in Von Plato, 1994, p. 217)
} 
where $p\left(H_{1} \mid H_{2}\right)$ reads "the probability of event $H_{1}$ given the event $H_{2}$ " and is called a conditional probability. More generally, the probability of $H$ given $D$ is the probability of the event $H$, given the reduced sample space $D$. Consider the Venn diagram on the left side of Figure II-2. The probability $p(H)$ can be viewed as the proportion of the sample space $\mathscr{S}$ that is occupied by $H$. The occurrence of the outcome $d$ has the effect of reducing the sample space $\mathscr{S}$ to the event $D$ because the elements outside $D$ are no longer possible outcomes (Book, 1977). The probability of $H_{1}$ is then the proportion of the event $D$ that is occupied by $H$, i.e., the conditional probability $p(H \mid D)$.

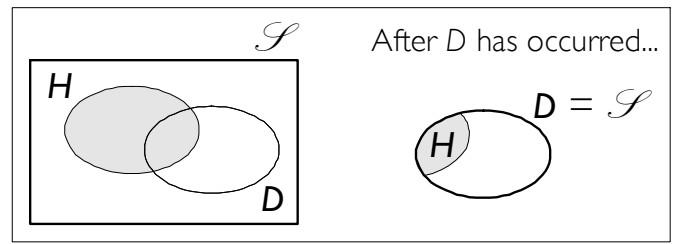

Figure II-2 - Venn diagram representation of the conditional probability of $H$ given $D$.

\section{Theorems of probabilities}

\section{Theorem 1}

If $H_{1}$ and $H_{2}$ are mutually exclusive events, then

$$
p\left(H_{1} \cup H_{2}\right)=p\left(H_{1}\right)+p\left(H_{2}\right)
$$

\section{Theorem 2 - Additivity principle}

If $H$ and $\sim H$ are complementary events, then,

$$
p(H)+p(\sim H)=1
$$

\section{Theorem 3}

Let $H$ be an event in a finite sample space $\mathscr{S}$. If $H=\varnothing$, then $p(H)=0$.

\section{Theorem 4 - Bayes's theorem}

Let $H$ and $\sim H$ be mutually exclusive, collectively exhaustive events of an experiment. If $D$ is an arbitrary event of $\mathscr{S}$ such that $p(D)>0$, then (as derived by Bayes, 1763/1958),

$$
p(H \mid D)=\frac{p(D \mid H) \cdot p(H)}{p(D)}
$$

The probability $p(H \mid D)$ is called the posterior probability of $H$ and corresponds to the probability of $H$ given the reduced sample space $D$. The probability $p(D \mid H)$ is called the likelihood of $D$ and corresponds to the probability of the event $D$ given the reduced 
sample space $H$. The meaning of these quantities will be further reviewed in the following section.

A second form of Bayes's theorem was derived by Laplace (see Howson \& Urbach, 1993) and reads as follows:

Let $H_{1}, \ldots, H_{n}$ denote $n$ mutually exclusive and collectively exhaustive events of an experiment. If $p\left(H_{i}\right), p(D)>0$ then

$$
p\left(H_{k} \mid D\right)=\frac{p\left(D \mid H_{k}\right) \cdot p\left(H_{k}\right)}{\sum_{i} p\left(D \mid H_{i}\right) \cdot p\left(H_{i}\right)}
$$

So, when $H$ and $\sim H$ denote two mutually exclusive and collectively exhaustive events or hypotheses, Bayes's theorem reads

$$
p(H \mid D)=\frac{p(D \mid H) \cdot p(H)}{p(D \mid H) \cdot p(H)+p(D \mid \sim H) \cdot p(\sim H)} .
$$

Finally, a third form of Bayes's theorem, found, e.g., in Savage (1954/1972), is called the "odds ratio" form of Bayes's theorem. It reads as follows:

Let $H$ and $\sim H$ be mutually exclusive, collectively exhaustive events of an experiment. If $D$ is an arbitrary event of $\mathscr{S}$ such that $p(D)>0$ and if $p(H), p(\sim H)>0$ then

$$
\frac{p(H \mid D)}{p(\sim H \mid D)}=\frac{p(D \mid H)}{p(D \mid \sim H)} \cdot \frac{p(H)}{p(\sim H)} \Leftrightarrow \Omega_{1}=L \cdot \Omega_{0} .
$$

where $\Omega_{1}$ represents the posterior odds in favour of $H$, while the prior odds in favour of $H$ are given by $\Omega_{0}$ and $L$ represents the likelihood ratio of $D$.

As already mentioned, the probability calculus is independent from the different existing meanings of the probability concept. The next section outlines the different meanings of probability, ranging from its earliest interpretation within Ancient Greek work to modern theoretical and philosophical developments.

\section{PROBABILITY AS A PHILOSOPHICAL CONCEPT}

\section{Origins of the probability concept}

\section{Probability in Antiquity and the Middle Ages}

Probability arose within the rhetorical framework of Antiquity (Schmitz, 2000). The first concept of probability was equivocal, encompassing both subjective beliefs and frequency considerations. For instance, Aristotle described probability as what "is, or is supposed to be, usually true" and as "that which happens usually but not always" 
(Rhetoric, 1402b, trans. 1984). Rhetorical probability encompassed what was culturally expected from human behaviour but also, what was normally expected from the natural world (Schmitz, 2000; see also O'Sullivan, 1995; Sheynin, 1974). In Medieval times, Aquinas directly referred to Aristotle's thoughts to define the meaning of a probable argument (Byrne, 1968; Kendall, 1956) as (i) widely accepted, (ii) based on the authority of the wise or (iii) a sign indicating where the truth lies rather than representing the truth itself. As such, probability was used to denote proposals that were not properly scientific (Byrne, 1968; Hacking, 1975).

This dogmatic conception of true knowledge was challenged in the $16^{\text {th }}$ century, when sceptics introduced the concept of subjectivity and rejected any type of knowledge, from demonstrative proofs, to sensory evidence (Gigerenzer et al., 1989). By the $17^{\text {th }}$ century, the 'reasonable' men adopted an intermediate pragmatic rationality: they cast out Aristotelian ideals of certainty and raised the merely probable to the standard of respectable knowledge (Popkin, 1964 as cited in Gigerenzer et al., 1989; Shapiro, 1983, ibid.; Daston, 1988). From this point in time, proofs of hypotheses were considered rational as long as they were sound enough to convince a sensible man to act in everyday life (Daston, 1988). Such a philosophical approach to rationality, completed with a new application of mathematics to new domains of experience, such as gambling and insurance, defines the historical context in which the mathematical concept of probability was to arise (Gigerenzer et al., 1989; Hacking, 1975).

\section{Emergence of the mathematical probability concept}

Pascal's gambling problem of points (Pascal, 1654/1963), and his correspondence with Fermat are often associated with the birth of the mathematical concept of probability (Hacking, 1975). Nevertheless, it was the concepts of stakes and expected gains rather than that of probability that were at the centre of Pascal's argumentation (Godfroy-Génin, 2000; Ranking, 1966). Hacking (1975) noted that Arnauld and Nicole $(1662 / 1992)$ were the first authors to finally connect probability with the notion of the measurement of randomness. These authors contrasted the probability of an event with its consequences, and warned players that a high prospective gain should be distinguished from the probability that this gain will occur. Once more, the concept of probability was linked with both subjective beliefs and frequency considerations. Yet the novelty was the suggestion that only the latter could form a 'reasonable' basis for evaluating probabilities. 
The relationship between natural frequencies and probability suggested by Arnauld and Nicole (1662/1992) was later formally demonstrated by Bernoulli (1713/1987). Bernoulli had to justify the fact that probability could be numerically conceptualised, at a time where the concept was still understood as an opinion from authority (Shafer, 1996). Bernoulli first laid the grounds for an explicit distinction between subjective and objective aspects of probabilities (Hacking, 1975) even though the author did not himself explicitly differentiate those two types of probabilities (Bernoulli, 1713/1987; see also Daston, 1988; Hacking, 1975). Bernoulli proposed that events whose certainty was objectively observable manifested the truth of the present or future existence of these events. In contrast, events whose certainty was subjectively observable relied on our knowledge of this truth. He proceeded to define probability as a degree of certainty and proposed to measure the probability of a given event by evaluating the number and the weight of its subjective and objective proofs (Bernoulli, 1713/1987).

\section{The probabilities of causes}

So far, the present review has focused on the development of the different bases for the interpretation of the concept of probability. As the mathematical concept of probability took shape, however, it also entered the realm of another philosophical debate, namely the discussion of scientific methods for explaining phenomena. This discussion was centred on the contrast between deductive and inductive methods of reasoning. In particular, the uncertainty associated with inductive reasoning-defined as the inference of causes from observed effects-led to a shift in the focus of the treatment of probabilities in the $18^{\text {th }}$ century (Daston, 1988). Scholarly discussions started to concentrate on the formalisation of the relation between the probabilities of causes; that is a priori probabilities, and a posteriori probabilities, which are conditioned on empirical observations (Daston, 1988; Hacking, 1975).

Bernoulli's work $(1713 / 1987)$ represents the first significant development with regards to the inference of the probability of a posteriori observations on the basis of known a priori probabilities of causes. The author indeed demonstrated the first limit theorem of the probability calculus (e.g., see Daston, 1988). Bernoulli (1713/1987) thus proposed the first concrete model of deductive causation, which illustrated how $p$ (effect $\mid$ cause) could be inferred from the knowledge of $p$ (cause). Yet he hoped that his result would provide the grounds for modelling inductive reasoning; that is, for demonstrating how $p$ (cause|effect) could be inferred from the knowledge of $p$ (effect). 
He, however, stopped short of demonstrating the inverse corollary of his theorem (Todhunter, 1865/1949).

It is not until the publication of Bayes's (1763/1958) essay that the final step towards the formalisation of inductive reasoning was taken (Fienberg, 1992). These grounds for probabilistic inductive reasoning were later also independently achieved by Laplace (1774/1986; see, e.g., Howson \& Urbach, 1993). Bayes's (1763/1958) theorem was presented by Price (in Bayes, 1763/1958) as the result of a quest for a method allowing the evaluation of the probability that an event will happen, on the sole basis of the knowledge of the frequency with which it happened and failed to happen in the past. Price (in Bayes, 1763/1958) pleaded that only Bayes's theorem provided the means of accurately measuring the strength of an inductive argument in contrast to common sense, which is constrained with vagueness and is often erroneous.

The first section of Bayes's essay contained the proof of the so-called Bayes's theorem, which is still one of the theorems of modern probability calculus (see pp. 1617). In the second section of his essay, Bayes generalised his theorem to situations where the prior probability of the cause is unknown; thereby demonstrating the inverse of Bernoulli's theorem. To do so, Bayes assumed unknown prior probabilities to be equally distributed. Bayes was concerned that this assumption "might not perhaps be looked upon by all as reasonable" (Price, in Bayes, 1763/1958). This assumption, later labelled the Principle of Indifference (Keynes, 1921; as cited, e.g., in Gillies, 2000), has indeed been widely disputed and was in fact to become the main source of the refutation of Classical theories of probabilities, but not before almost a century had elapsed.

\section{The Classical interpretation of probability}

\section{Origins of the Classical interpretation}

It is the work of Laplace (e.g., 1814/1951) that widely introduced the Classical interpretation of probability to philosophers and mathematicians in the $19^{\text {th }}$ century (e.g., Howson \& Urbach, 1993). The Classical approach and more specifically its later rejection played a crucial role in the development of modern theories of probabilities, and the first explicit distinction between objective and subjective probabilities. Laplace argued that probability was not inherent in Nature (itself subject to a universal determinism). Instead he conceived probability as a measure of human ignorance and argued that this ignorance should lead the reasonable man to consider all events to be equally possible, thereby positing what has later been referred to as the Principle of 
Indifference (Gillies, 2000). Consequently, Laplace proposed that the probability of an event should be evaluated by "the ratio of the number of favourable cases to that of all the cases possible" (Laplace, 1814/1951, p. 11; see also Gillies, 2000).

This interpretation remained unchallenged for nearly a century even though clear finite sets of equally probable cases are quite rare in the real world (Gillies, 2000; Hacking, 1975). In fact, Classical probabilists saw no contradiction between the argument that probability corresponded to the degree of certainty held by reasonable men on the one hand, and the fact that probability judgements should also reflect longrun distributions of events in nature and society (Porter, 1986). Daston (1988) argued that this apparent paradox was made possible by the work of philosophers such as Hume (e.g., 1739/2000) who proposed that our subjective beliefs are proportional to the "vivacity" of our mental representations of external events, themselves mirroring objective experience.

\section{Decline of the Classical interpretation}

Statistical observations ultimately led to the development of the Frequency theory of probability, based on purely objective considerations (see, e.g., Daston, 1988; Fienberg, 1992; Shafer, 1990; Von Plato, 1994). For instance, Daston (1988) observed that Cournot retained the Classical framework for the treatment of probability but implemented it with statistical considerations. Other authors (e.g., Ellis, 1849; Mill, 1843/1973) attacked the Principle of Indifference and argued that equipossibility should be established empirically rather than assumed to be true. The development of the Frequency theory of probability followed the exposition of those criticisms and was first fully presented by John Venn (1866/1962).

The grounds of Classical probability thus started to crumble from the middle of the $19^{\text {th }}$ century onwards. On the one hand, Frequentists undermined the assumption of universal determinism when they argued that only empirical observation could inform us of the laws of Nature (Gillies, 2000). On the other hand, the claim that (Classical) probability theory was an accurate description of the enlightened man's good sense was more and more weakened by the multiplication of mathematical paradoxes where settled mathematical solutions were at odds with good sense (Daston, 1988; Howson \& Urbach, 1993). Howson and Urbach (1993) provided a compelling example of such paradoxes, 
Consider an urn again. Suppose that you draw a ball from an urn which you are told contains white and coloured balls in some unknown proportion, and that the coloured balls are either red or blue. What sort of ball will you draw? Your data seem to be neutral, in the first instance, between its being white or coloured. Hence according to the Principle of Indifference, the probability that it will be white is one half. But if it is coloured, the ball is red or blue, and the data are surely neutral between the ball's being either white or blue or red. Hence, according to the Principle of Indifference, the probability of the ball's being white is one third. (Howson \& Urbach, 1993, p. 59)

\section{Modern conceptions of probability}

With the rejection of the Principle of Indifference, came the dissociation of subjective and objective aspects of probability (Hacking, 1975). This dissociation in turn led to the development of various different modern conceptions of probability. This section briefly reviews the main existing modern interpretations of probability, ranging from the Frequentist (e.g., Von Mises, 1928/1957) to the Subjective approach (e.g., De Finetti, 1937/1964), via "intermediary" positions such as the Logical approach to probability, developed by Keynes, or else the Propensity approach, developed by Popper (see, e.g., Gillies, 2000).

\section{Frequentist conception of probability}

Von Mises (1928/1957) further developed the Frequentist interpretation of probability introduced by Venn in the late $19^{\text {th }}$ century. Von Mises (1928/1957) agreed with Venn and his predecessors that Classical probabilists failed to demonstrate how one should proceed from a priori assumptions concerning equally possible cases to the description of natural events. He argued that probability must be quantitatively assessed with infinite sequences of observations. Thus, from the Frequentist perspective, the probability that $x$ has the attribute $A$ is the limit of the relative frequency of As among $x$; that is,

$$
p(A)=\lim _{x \rightarrow \infty} \frac{n(A)}{n(x)}
$$

Since, according to this view, degrees of probability are empirically determined, a probability statement such as 'there is a $p$ degree of probability that hypothesis $h$ is true' is characterised as an empirical statement about hypothesis $h$, based on observational evidence (Kyburg \& Smokler, 1964). The Frequentist approach precludes the application of probability to single-case events or propositions because they do not frequently repeat themselves. Von Mises (1928/1957) distinguished between ordinary and scientific or rational interpretations of probability. In ordinary language, one may 
speak, for instance, of 'the probability that an accused person is guilty.' Von Mises argued that these expressions can only be interpreted on a descriptive level whereas the Frequentist definition of probability is no longer descriptive but true.

Although Frequentist probabilities are often accepted as the 'true' or 'scientific' interpretation of probability, not all scholars agree that probability statements should or can have such an empirical status. Upholders of the Logical approach to probabilities, for instance, take "probability as representing a logical relation between a proposition and a body of knowledge" (Kyburg \& Smokler, 1964, p. 5).

\section{Logical conception of probability}

The Logical conception of probability was first fully developed by Keynes (1921, as cited by, e.g., Gillies, 2000). According to the rules of formal logic, a deductive inference leads to a certain conclusion that is fully entailed by its premises. For example, if we know that (i) the occurrence of $A$ entails the occurrence of $B$, and that (ii) $A$ has occurred, we can deduce with certainty that $B$ has occurred, according to the modus ponens rule. Keynes proposed to generalise the principles of deductive logic to inductive inferences where the occurrence of A only partially entails that of B (Gillies, 2000; Howson \& Urbach, 1993).

It follows that Logical probability is the measure of the extent to which a set of premises confirms the truth of the conclusion, viz., the measure of the degree of partial entailment (Gillies, 2000; see also Savage, 1954/1972, p. 3). Gillies (2000; see also Ramsey, 1931/1964) noted that Keynes further assumed that if A partially entails B with a probability $\alpha$, then it is rational to believe that when $A$ has occurred, the probability that $B$ will also occur equals $\alpha$. In other words, Keynes's concept of probability encompassed both the notion of degree of entailment and that of rational and objective belief, thereby distinguishing Logical probabilities from Subjective probabilities (Gillies, 2000). As Gillies (2000) further noted, Keynes's reference to an objective basis of probability should also be distinguished from the notion of material objectivity used by the advocates of the Frequency approach: in Keynes's work, objectivity is not empirical, but rather related with Plato's hypothetical world of objective and abstract ideas.

Another characteristic of Keynes's Logical probability theory is the argument that Logical probabilities cannot be measured unless they originate from a well-defined set of equiprobable alternatives, thereby endorsing Laplace's Principle of Indifference. Keynes modified the Principle in the attempt to resolve the paradoxes it entailed: the author 
proposed to limit the application of the Principle of Indifference to cases where the alternatives were in finite number and could not be partitioned into sub-alternatives. Yet this modification was not sufficient as more paradoxes were identified, and are still unsolved to this date (e.g., see Gillies, 2000, p. 49).

\section{Subjective theory of probability}

In the late 1920s, Bruno de Finetti in Italy and Frank Ramsey in England independently developed a new Subjective approach to probability. This Subjective approach was concerned with the development of a psychological measurement of degrees of belief where the probability that hypothesis $H$ is true relative to the body of evidence $D$ is not uniquely determined (e.g., Howson \& Urbach, 1993). Thus, in contrast with the Frequentist and Logical views, the Subjective approach stipulates that each individual may have different degrees of belief in the truth of a statement or in the occurrence of an event, relative to the same independent body of evidence-whether or not this body of evidence is based on a given sequence of observation (e.g., see Kyburg \& Smokler, 1964).

To measure degrees of beliefs, subjectivists such as De Finetti (1937/1964) proposed to use betting ratios. De Finetti (1937/1964) defined individuals' Subjective probabilities as the ratio of the sum those individuals would agree to bet on an uncertain event $H$ (denoted by ' $p \times S$ ') to the minimum return of sum $(S)$ they would require for agreeing to bet in the first place. For instance, if you would only agree to bet $£ 1(p \times S)$ on a Greyhound in a dog race under the condition that you could get $£ 3$ (S) back, then your Subjective probability $p$ that the dog will win the race is $\frac{1}{3}\left(\frac{p S}{S}\right)$. The more money you would want in return before you agree to bet $£ 1$, the lower your Subjective belief that the dog will win.

From the Subjective perspective, there are no restrictions on the subjective degree of belief held for a particular alternative. Restrictions, however, do apply to the way beliefs are 'distributed' among related alternatives (Kyburg \& Smokler, 1964): actions or bets can only be rational if they are coherent, viz., if they do not entail a loss of return on all possible outcomes. De Finetti (1937/1964) and Ramsey (1931/1964) demonstrated what is now called the Ramsey-De Finetti theorem. This theorem states that a sufficient and necessary condition for coherence is that degrees of belief conform to the rules of probability calculus (see Gillies, 2000, pp. 60-64 for a proof of this theorem). 
It is worth noting that subjectivists never intended their theory to be taken as a descriptive theory of human probabilistic judgements. The Subjective approach presents the normative requirements to which rational judgements under uncertainty should conform. Subjectivists thus denied that the fact that actual judgements are not coherent would bear any consequences on the validity of the Subjective theory of probability itself (De Finetti, 1937/1964; see also Kyburg \& Smokler, 1964). Also, whereas Frequentists argued that probabilities based on single events were only descriptive and could not be assimilated to the true probability of an event, De Finetti (1937/1964) argued against the existence of objective probabilities based on relative frequencies of occurrence of outcomes. The author indeed suggested that even experimental outcomes were in fact single events, and concluded that probabilities could only be understood with reference to single events. In the attempt to argue the case for the concept of objective probabilities based on single outcomes, another framework of interpretation, called the Propensity theory of probability, was later developed by Popper (1957b, as cited by Gillies, 2000).

\section{Propensity theory of probability}

To conceptualise the notion of objective single-event probabilities, Popper (1959, as cited by Howson \& Urbach, 1993) argued that sequences of repetitive observations possess dispositions or propensities to produce a certain relative frequency, just as, for example, gold has a physical propensity to shine. It follows that probability is a dispositional property of a particular experiment and the objective probability of a single outcome is equal to the relative frequency of occurrence of similar outcomes observed on completion of the experiment. (Gillies, 2000; Howson \& Urbach, 1993). Howson and Urbach (1993; see also Gillies, 2000) however argued that Popper's interpretation of probability corresponds to a Subjective evaluation of a single-case probability based on objective considerations rather than an objective evaluation of a single-case probability in itself. Gillies (2000) illustrated this criticism with reference to the reference class problem whereby "the probability [of a single event] depends on how the event is categorised rather than on the event itself," (Gillies, 2000, p. 120). Hence, such a probability cannot be objective, by definition.

In sum, the review of the different interpretations of probability, which have arisen from Antiquity until our modern era allows a better understanding of the tensions existing between the subjective and objective facets of this concept, as well as their 
origins. Despite the diversity of philosophical interpretations of probability, there is a wide consensus on the probability calculus, i.e., the mathematical rules-as first exposed by Kolmogorov in 1933-that should govern probabilities, regardless of any pragmatic meaning one attributes to them. A direct consequence of the probability axioms is Bayes's theorem, which introduced the concept of posterior probability in relation to the problem of induction in causal reasoning. As anticipated by Classical authors such as Bernoulli (1713/1987), posterior probability judgements have played an important role in everyday life, such as in medical or legal settings, for instance. The question of how we make posterior probability judgements has become one of the focuses of Psychological research since the 1960s.

The following section reviews the interpretations of probability within the Psychological Sciences and examines the relation between past and current theoretical debates surrounding posterior probability judgements and "psychological" concepts of probability. This examination will in turn reveal both a lack of empirical investigation of individuals' psychological probability concepts and questionable assumptions regarding what ought to constitute the normative basis for such concepts.

\section{CONCEPT(S) OF PROBABILITY WITHIN PSYCHOLOGY}

\section{Theoretical taxonomies of psychological probabilities}

The preceding historical review illustrates the several meanings that scholars have associated with the concept of probability. Yet to the psychologist whose aim is to study probability judgement, the critical issue becomes the appraisal of lay individuals' interpretation and understanding of this multi-faceted concept. This section reviews the different psychological accounts of naïve probability concepts ${ }^{2}$. This in turn reveals a striking resemblance between the tensions surrounding the philosophical interpretations of the concept and those surrounding the theoretical considerations of the bases of human naïve interpretation(s) of probability.

\footnotetext{
${ }^{2}$ This review focuses on concepts underpinning the interpretation of numerical probabilities, which are the focus of this thesis. The literature also considers the use and interpretation of verbal probabilities (e.g., see Budescu \& Wallsten, 1985; Teigen, 1988; Teigen \& Brun, 1995, 2003; Wallsten, Budescu, Rapoport, Zwick, \& Forsyth, 1986). The present review, however, will be limited to those aspects of the literature that are directly relevant to the issue at hand, namely, the psychological characterisation of the concept of (numerical) probability, with a view to later inform the computational analysis of numerical posterior probability judgements.
} 
In 1954, Vail proposed a taxonomy of different types of probability by distinguishing mathematical and objective probabilities from psychological (Subjective) probabilities. He defined Subjective probabilities as referring "to propositions concerning contemplated [past, present or future] events" (p. 90) and encompassing various concepts such as degree of belief, psychological probability and perception of frequency. Vail's concept of mathematical probabilities $(\mu)$ is close to Kolmogorov's 'skeleton' concept, which is independent (and applies to all) of the philosophical interpretations of probability (see p. 13). Objective probability $(f)$ can adequately be identified with Von Mises's concept of probability and was defined by Vail as "the objective relative frequency of an empirical event... [presupposing] knowledge whether the event did in fact happen, a suitable operative definition of time intervals and an objective method for counting occurrences and non-occurrences" (p. 92).

Vail (1954) defined three different types of psychological probabilities: $\psi \mu$ or perception of $\mu, \psi f$ or perception of $f$ and finally, $\psi \beta$ or degree of belief. ${ }^{3} \psi \mu$, Vail proposed, is "not only a function of $\mu$, but a function of the particular event $E$, whose $\mu$ is under consideration; it will be a function of the wording of the problem, of time allocated to its solutions, and so forth" (pp. 92-93). He further defined $\psi f$ as "the impression we obtain by being exposed to the occurrence of a finite repetitive event" (p. 93), and $\psi \beta$ as the degree of "confidence in the occurrence of an event ... [which] may or may not have arisen from a conscious attempt to calculate the [mathematical probability] $\mu \ldots$ of the event, or to observe the [objective relative frequency] $f$ (if any) of the event" (p. 93). This latter account of $\psi \beta$ suggests that people may sometimes evaluate probability on the basis of different types of information ( $\mu, f$, or some "other" bases). Yet the question of how these elements would be combined to form the judgement outcome or what underlies $\psi \beta$ judgements when they are not based on $\mu$ or $f$ considerations, remains open. Finally, Vail suggested that the judgements we observe may be a distorted image of people's actual probability as a result of the influence of psychological unknowns such as the format of the information presented, the time pressure of the situation, or the utility of the proposition considered. He concluded that only actual estimates, devoid of the

\footnotetext{
${ }^{3}$ Vail (1954) did not distinguish between $B$ and $\psi \beta$; this distinction was added here to prevent the confusion between the theoretical notion of degree of belief introduced by the subjectivists and actual judgements, in line with De Finetti's remark (1937/1964, p.111, note (e)).
} 
influence of those psychological unknowns, should be tested against normative standards.

John Cohen $(1960,1972)$ distinguished between a psychological interpretation of probability on the one hand, and the Classical, Logical and Frequentist interpretations of probability on the other hand. In contrast to the different types of psychological probabilities proposed by Vail (1954), Cohen proposed only one concept of psychological probability depending on different types of information (statistical, factual, and personal). Although his conception of psychological probability was close to the Subjective approach, Cohen insisted that psychological probability should be conceived as a phenomenon in its own right, independent of any axiomatic norms, and possibly underlying, in a systematic way, behaviours under uncertainty such as risk-taking, guessing, predicting, and decision-making.

The effect of the format of the information suggested by Vail (1954) was further conceptualised by Howell and Burnett (1978). These authors proposed that the different bases of people's probability judgements might indeed be determined by the tasks used to measure uncertainty. In particular, Howell and Burnett (1978, p. 55) suggested that probability judgements are derived by a combination of four cognitive elements: prior knowledge or belief in the generating process giving rise to the uncertain event, stored frequency data, heuristics, and systematic biases such as the "selective bias" whereby information is selectively attended to and the "confidence bias" whereby probability judgements of events may be influenced by individuals' internal attribution of the event's cause. Howell and Burnett (1978) proposed that the type of judgement (e.g., frequency vs. probability estimation), alongside with information about the event to be judged (e.g., frequentistic vs. nonfrequentistic ${ }^{4}$; (un)known external vs. (un)practiced internal generator) would affect the type of cognitive elements involved. Yet the authors themselves recognised the speculative nature of the conclusions they derived from their taxonomy and called for more empirical research.

In line with Howell and Burnett's distinction between external and internal generators of uncertain events, Kahneman and Tversky (1982/1982d) proposed a

\footnotetext{
${ }^{4}$ The authors' taxonomy was nevertheless biased towards a frequentistic conception of probability as they argued that, "The task of estimating the probability of nonfrequentistic events makes little apparent sense from a logical standpoint except insofar as people are able to estimate by analogy with frequentistic events." (Howell \& Burnett, 1978, p. 62)
} 
taxonomy of uncertainty that distinguishes between external and internal uncertainty. They defined external uncertainty as the uncertainty attributed to the external world whereas internal uncertainty was defined as the uncertainty attributed to the state of our knowledge. According to the authors, external and internal uncertainties have different bases of assessments. External uncertainty can be based either on singular evidence or on distributional evidence. Kahneman and Tversky (1979/1982a) defined singular evidence as "evidence about the particular case under consideration" and the distributional evidence as "knowledge about the distribution of outcomes in similar situations" (p. 414). They linked assessment of external uncertainty based on distributional data to the Frequentist interpretation of probability. In contrast, these authors proposed that internal uncertainty was either directly evaluated on the basis of an "introspective judgement of the strength of an association [based on one's experience]" (Kahneman \& Tversky, 1982/1982d, p. 519) or evaluated through a "[reasoned] process of sifting and weighing of evidence and arguments" (ibid.). They linked any assessment of internal uncertainty to the Subjective interpretation of probability.

The authors insisted that their taxonomy was not mutually exhaustive. They conceived that uncertainty could be both internal and external, i.e. attributed both to external dispositions and to the extent of one's knowledge, as well as assessed by a combination of different types of evidence (singular, distributional, introspective and/or reasoned). Finally, Kahneman and Tversky (1982/1982d, p. 519) argued that the laws of the Probability Theory may not equally apply to all variants of uncertainty. Thus, they proposed that intuitive judgements of external uncertainty based on distributional evidence were likely to be in line with the rules of probability calculus. In contrast, they suggested that judgements based on singular evidence (external uncertainty), introspective evidence or reasoned arguments (internal uncertainty) may not be constrained by the additivity principle. Despite this argument, the authors attributed posterior probability judgements' deviations from normative prescriptions to biases in human judgements rather than to differences in the type of uncertainty evaluated (see next subsection, p. 31)

Another taxonomy was recently developed by Teigen (1994). He proposed that there are a number of different processes underlying subjective judgements of probability, and distinguished intuitive assessments from indirect or rule-dictated assessments. Intuitive assessments are defined as based on argument weighting, degrees 
of convictions or knowledge or judgemental heuristics. By contrast, rule-dictated assessments refer to assessments relying on (quasi-)analytical rules whose appropriateness is determined by the extent of individuals' grasp of the probability calculus. Teigen (1994) thus identified five rules as possible candidates that would generally be accepted as valid by lay people. Table II-1 summarises these rules, in increasing order of difficulty.

Table II-1

Teigen's (1994) taxonomy of quasi-analytical and analytical rules that may be used as a basis for probability judgements

\begin{tabular}{ll}
\hline Rule & Description \\
\hline $50 / 50$ & $\begin{array}{l}\text { When two outcomes or alternatives appear equally possible, their } \\
\text { probability is } 50 .\end{array}$ \\
& $\begin{array}{l}\text { The probability of an outcome or alternative is a/n where } a \text { is the } \\
\text { number of similar outcomes/alternatives and } n \text { is the total number of } \\
\text { outcomes/alternatives considered. }\end{array}$ \\
& $\begin{array}{l}\text { When } n \text { outcomes or alternatives appear equally possible, their } \\
\text { probability of one occurring is } 1 / n .\end{array}$ \\
Relative frequency & $\begin{array}{l}\text { The probability of an outcome occurring in the future is equal to its past } \\
\text { relative frequency of occurrence. }\end{array}$ \\
Complementarity & Corresponds to the additivity principle.
\end{tabular}

With regards to the intuitive evaluations of uncertainty, Teigen (1994) followed Kahneman and Tversky's (1982/1982d) distinction between external and internal uncertainty. But, rather than further distinguishing these categories with the evidence on which they can be based, Teigen (1994) proposed to distinguish them on the basis of their emphasis. The author indeed argued that an emphasis on the fact that the different alternatives are indeterminate will trigger judgements based on chance probabilities when uncertainty is external. But, when uncertainty is internal, the same emphasis will entail judgements that reflect our uncertainty by ignorance. In contrast, an emphasis on the tendency of an outcome to occur will elicit the use of dispositional probabilities if uncertainty is external and the use of estimates of confidence if uncertainty is internal.

Despite the clear potentials of existing classifications for advancing our understanding of probability judgements, a common characteristic of these accounts is their lack of empirical validation. This, in turn, leaves open the issue of what actually constitutes the psychological interpretation of probability underlying any given judgement. Instead of investigating people's interpretations of probabilities, research on 
probability judgements has traditionally focused on the study of deviations from the normative rules of the probability calculus, thereby positing what interpretation participants ought to hold when making judgements under uncertainty, and in particular posterior probability judgements.

\section{Probability in posterior probability judgements}

In view of the preceding review of existing taxonomies of psychological concepts of probability, one may anticipate that these taxonomies would form the basis for studying posterior probability judgements. Yet normative considerations have prevailed over such a descriptive approach. When, in the early 60s, psychologists' attention was originally drawn to posterior probabilities and their judgement, the distinction was made between $\beta$, often assimilated with Savage's (1954/1972) ideal personal probabilities and $\psi \beta$, namely participants' probability judgements (e.g., see Edwards, 1961).

Edwards, Phillips, Hays, and Goodman (1968, see also Edwards, 1968) defined $\beta$ as a measure of uncertainty varying between zero and one representing "the extent to which a somewhat idealized person believes a statement to be true.” (p. 250). By somewhat idealized, the authors meant that they assumed that this person's probabilities followed the law of addition of probability and its consequences, and noted that few actual persons could conform to all the consequences of the law of addition (Bayes's theorem being one of them). This definition, the authors argued, allowed the concept of probability to be applied to single-case events and also to events whose relative frequency could be ascertained (Edwards et al., 1968). This view represents quite an accurate definition of $\beta$ probabilities. In practice, however, those earlier studies on human probabilistic judgements relied on a comparison of $\psi \beta$ estimates with values of $\beta$ solely based on the relative frequency of the events contemplated (e.g., see Phillips \& Edwards, 1966; Phillips, Hays, \& Edwards, 1966).

In a much similar vein to Von Mises (1928/1957) who compared the scientific measure of probability to that of a physical entity, Tversky and Kahneman (1974/1982b) compared psychological judgements of probabilities with that of physical quantities. These authors, who initiated the heuristics and biases framework of research, argued that probability judgements were subject to biases in the same way judgements of distance could be biased as a function of the level of clarity at the time of judgement. The heuristics and biases framework entailed a shift of paradigm from that used by Edwards and his colleagues in the 60s. Yet the 'objective' conception of probability 
based on Frequentist considerations continued to play a crucial role in this new framework of research: probability judgement biases were indeed defined in terms of judgements' deviations from 'objective' norms based on relative frequencies. For instance, Tversky and Kahneman (1974/1982b) described prior probabilities as the base rate frequency of the outcome which they illustrated as follows:

... consider an individual who has been described by a former neighbor as follows: "Steve is very shy and withdrawn .... he has need for order and structure, and a passion for detail." ... the fact that there are many more farmers than librarians in the population should enter into any reasonable estimate of the probability that Steve is a librarian rather than a farmer. (Tversky \& Kahneman, 1974/1982b, p. 4)

In other words, the authors argued that reasonable individuals should primarily base their Subjective probability that Steve is a librarian on the estimate of the objective probability defined by the relative frequency of librarians.

Yet one could contend that it is not clear whether the uncertainty related to the statement 'Steve is a librarian' is external or internal (in reference to the authors' own taxonomy of uncertainty as described pp. 28-29 above). In other words, individuals may assess uncertainty internally; that is, they may evaluate the extent to which their knowledge can determine whether Steve truly is a librarian. Alternatively, individuals may attempt to assess uncertainty externally; that is, they may evaluate the extent to which the event in question would occur, given their knowledge of the distribution of farmers and librarians whose description matches that of Steve. According to Kahneman and Tversky (1982/1982d) themselves, the rules of probability only straightforwardly apply in the latter case, i.e., under the prerequisite that judgements are based on an assessment of external uncertainty.

Paradoxically, this prerequisite has rarely if ever been tested in studies of posterior probability judgements. Instead, comparison with normative standards is customary, warranted by the assumption that 'reasonable' individuals ought to attribute uncertainty to external factors (see above). But this requirement is only valid under a strict Frequentist interpretation of the meaning of probability. And even then, such interpretation would require knowledge of the actual relative frequencies of events to warrant the evaluation of judgement accuracy. Alternatively, from a subjectivist perspective, to evaluate the extent to which Steve 'truly' is a librarian in view of his description is no less reasonable than to base one's probability judgement on external distributional evidence. 
Kahneman \& Tversky (1972/1982c) contrasted subjective and objective probabilities: they argued that subjective probabilities corresponded to individuals' judgements whereas the objective probabilities were calculated according to the laws of the Probability calculus. The authors insisted that their approach was deliberately noncommittal with respect to philosophical meanings of probability. Thus, what they referred to as "subjective probability" should be clearly distinguished from the philosophical concept sharing its name, and preferably identified with a psychological concept of probability $(\psi)$, bearing no assumptions as to whether it actually follows the axioms of the probability calculus.

Yet the concept of "objective probability" generally used as a standard of comparison in studies of posterior probability judgements, and thus necessary to the definition of biased judgements, can only be calculated using frequentistic information. Whereas this assumption has rarely been challenged within research on posterior probability judgements, the equation of a normative conception of probability with its Frequentist conception has yet to receive a unanimous support from philosophers. As indicated by the earlier review of the different existing conceptions of probability, many have questioned the assumption that a probability based on objective data can be defined as objective (see also Cohen, 1979; Hogarth, 1975; Howson \& Urbach, 1993, p. 422). Indeed, we saw that, according to the Subjective conception of probabilities, degrees of belief ought to be coherent within one individual's set of beliefs; that is, ought to respect axioms of probability theory, such as the additivity principle, for example. This approach does not make any assumptions with regards to the correspondence of any particular belief; its relation to external statistical data or its empirical accuracy (Savage, 1954/1972, p. 68; see also Hammond, 1996/2000, for a discussion of the distinction between correspondence and coherence approaches).

This argument has been echoed by Phillips (1970) who argued that experimenters should acknowledge that, by using the relative frequency of events as a reference for evaluating $\psi \beta$, they are only assessing how participants perceive the reality experimenters have themselves identified. Phillips (1970), in line with Cohen (1960, 1972), argued in favour of an investigation of the psychological processes underlying probability judgements, that would be free of reference to "any particular characteristic as the 'objective' basis for judgement" (p. 257). He proposed to set a research agenda for investigating how judgements under uncertainty are affected by stimulus characteristics, 
past experience, prior knowledge, social and cultural norms, and, finally how the cognitive processes underlying judgements are affected by the context in which judgements may take place. Yet the advent of the heuristics and biases framework somewhat excluded such a "reference-free" study of probability judgements.

\section{CONCLUSIONS}

The review of the different interpretations of probability, which have arisen from Antiquity until our modern era allows a better understanding of the Janus-faced (Hacking, 1975) characteristic of this concept. The concept of probability has indeed consistently combined objective and subjective considerations since Antiquity up until the early developments of mathematical probability. It is only when new statistical sciences emerged in the $19^{\text {th }}$ century that the epistemological facet of probabilities, based upon subjective knowledge, was distinguished from their frequentistic attributes arising from statistical data. Given the objectivity of statistical law, Frequentist probability is often taken to express the true likelihood of occurrence of an event, on the basis of its observed relative frequency of occurrence.

Alternatively, the Subjective approach rejects the idea of a true probability. Subjectivists argue that probability is intrinsically subjective and relative to individual opinions. From this latter perspective, the accuracy of the probability measure is no longer a meaningful criterion for evaluating probabilities, and one needs not to make infinite repetitions of observations to make a rational probability judgement. Rather, probabilities need to be coherent, i.e., in line with the axioms constituting the probability calculus, and can be applied to single-case events. Propensity theory recently attempted to raise the case for an objective conception of single-case probabilities although this can be reduced to a Subjective interpretation based on objective considerations.

Despite the diversity of philosophical interpretations of probability, there is a wide consensus on the probability calculus that should govern probabilities, independently of any pragmatic meaning one may attribute to them. A direct consequence of the probability axioms is Bayes's theorem, which introduced the concept of posterior probability in relation to the problem of induction in causal reasoning. Posterior probability judgements play an important role in everyday life and the question of how we make posterior probability judgements has become one of the focuses of 
Psychological research. But the review of the interpretations of probability within the Psychological Sciences revealed a lack of empirical investigation of individuals' psychological probability concepts.

Moreover, with regards to the study of posterior probabilities, there remain contradictory accounts of the concept of psychological probability. Thus, Kahneman and Tversky (1982/1982d) proposed that individuals' judgements under uncertainty may reflect the assessment of internal or external uncertainty and that the laws of probability theory may only convincingly apply to judgements of external uncertainty. Yet when evaluating posterior probability judgements, the authors did not check whether a comparison with normative standards was warranted; that is, whether judgements were based on an assessment of external uncertainty. Instead they proposed that reasonable individuals would rely on assessments of external uncertainty (Kahneman \& Tversky, 1972/1982c). Even from a mere descriptive standpoint, there is disagreement with regards to what constitutes the psychological basis for probabilities. Recent developments in the field (see, e.g., Gigerenzer \& Hoffrage, 1995; Cosmides \& Tooby, 1996) proposed that the only concept of probability that humans can handle is based on absolute frequencies arising from natural sampling. In contrast, Koehler (1996) argued that one should distinguish subjective prior probabilities from objective (frequentistic) base rates: "prior probabilities may be informed by base rates, but they need not be the same" (p. 12). Keren and Teigen (2001) proposed that psychological probabilities were based on a Propensity interpretation whereas Oaksford and Chater (1998, 2001) related probability judgements with the Logical framework. But these frameworks are developed independently, and there is still little empirical evidence to show what interpretation(s) does underlie any given individual probability judgement.

Although these issues are rarely discussed in studies of posterior probability judgements, they are crucial to the furthering of our understanding of those judgements and the processes that underlie them. The next chapter will review the empirical studies of posterior probability judgements, from the earlier studies on conservatism up until the recent work on information formats (e.g., using natural frequencies). Posterior probability judgements involve updating one's prior judgement of the probability of an event in the light of new evidence where Bayes's theorem provides a normative model for calculating posterior probabilities. Thus, the study of posterior probability judgements not only concerns individuals' psychological concept(s) of probability, but also their probabilistic inferences. The discussion of the body of literature on posterior 
probability judgements will highlight researchers' prevalent concerns with participants' observed performances and examine the shortcomings of such perspectives, with regards to our understanding of what constitutes human probabilistic inference, using a processfocused perspective. Such discussion will, in turn, lay the ground for the presentation of the empirical research programme of the present thesis, followed by the presentation of a new theoretical account of posterior probability judgements.

\section{sk}


He who wants greater certainty, who does not want to make any predictions before he can believe with certainty in their coming true, cannot be helped.

(Reichenbach, 1932/1949, p. 323)

7 me main purpose of this thesis is to further develop our understanding of posterior probability judgements by delineating and developing the processfocused study of these judgements. The process-focused approach to the study of posterior probability judgements first entails the development of a rigorous computational account of people's judgement methods (i.e., identify what individuals actually do when making such a judgement). Such an account is then taken to form the basis for the development of an algorithmic account explaining the origin of the judgement methods thus elicited (i.e., identify how individuals do what they do when making a posterior probability judgement).

Hence, in line with a process-focused research agenda, the present chapter is aimed at reviewing the state of our knowledge concerning existing computational accounts of posterior probability judgement processes as well as existing algorithmic models of these judgements. Following a brief introduction to the historical context of the empirical study of posterior probability judgements, the review will be organised around the three main current experimental paradigms-namely, the Textbook-problem paradigm, the Social-judgement paradigm and the Set-based paradigm. The discussion of the body of literature on posterior probability judgements from a process-focused perspective will highlight issues that may have been overlooked, as they were not central to the outcome-focused study of probability judgements favoured for the past three decades. Yet these same issues are crucial to the process-focused study of posterior probability judgements and, as such, will form the impetus for the empirical research programme of the present thesis.

\section{CONCISE HISTORICAL CONTEXT TO THE STUDY \\ OF POSTERIOR PROBABILITY JUDGEMENTS}

\section{Conservatism studies}

Edwards (1962; see also Edwards, Lindman \& Savage, 1963) first introduced Bayesian concepts to psychologists within the context of Dynamic Decision Ttheory, 
which was concerned with sequences of decisions where decision-makers can apply in subsequent decisions what they might have learnt in previous ones. Bayes's theorem and Bayesian statistics provided, in conjunction with the maximisation of expected value, a normative model prescribing how one ought to combine old and new probabilistic information (Edwards, 1962). Research on posterior probability judgements that followed Edwards' exposé focused on the revision of opinions and assessed individuals' performance by comparing the value of their judgements with those provided by Bayes's theorem.

Those earlier studies mostly relied on a book-bag-and-poker-chips paradigm, which consisted in presenting participants with problems involving book bags filled with specified proportions of blue and red poker chips (e.g., see Peterson, Schneider, \& Miller, 1965; Phillips \& Edwards, 1966). Typically, the setting involved two bags with symmetrical compositions of blue and red chips in unequal proportions. One bag was selected at random and participants were presented with several samples of balls drawn from the selected bag. After each sample, participants were asked to infer the posterior probability that the bag contained a majority 'red' or 'blue' chips in the light of the sample observed. Their judgement was recorded, the balls replaced in the bag and another sample was drawn.

The first studies on conservatism (e.g., Phillips \& Edwards, 1966; Phillips, Hays, \& Edwards, 1966) revealed that individuals' revisions of probabilities favoured the same hypothesis as that implied by posterior probabilities evaluated using Bayes's theorem. Nevertheless, participants tended to be conservative in their probability estimates, viz., their posterior probability judgements were not as extreme as their normative counterparts. Several procedural variables were manipulated to investigate the extent of the conservatism effect (for reviews see Rapoport \& Wallsten, 1972; Slovic \& Lichtenstein, 1971).

Phillips and Edwards (1966; see also Edwards, 1968) developed a simple analytic model to describe participants' conservative judgements, which formed the basis for the theoretical explanation of the conservatism effect (for reviews of these accounts, see Edwards, 1968; Rapoport \& Wallsten, 1972; Slovic \& Lichtenstein, 1971). The model accurately described mean outputs of posterior probability judgements, but several authors questioned the extent to which this should also be accepted as evidence for the psychological processes underlying individual judgements. In seminal reviews, Slovic and Lichtenstein (1971) and Rapoport and Wallsten (1972) argued that our understanding 
of individuals' information processing strategies indeed could not be adequately achieved on the basis of a description of aggregated individuals' judgement outputs or on the departure of those aggregated outputs from normative prescriptions. These authors consequently called for the development of descriptive models of the "heuristic strategies that subjects employ when they integrate information" (Slovic \& Lichtenstein, 1971, p. 729).

\section{Heuristics and biases in judgements under uncertainty}

Slovic, Fischhoff, and Lichtenstein (1977) contended that the awaited development of our understanding of such heuristic strategies was successfully initiated by the decisive work of Daniel Kahneman and Amos Tversky. These latter authors' original focus of research was no longer the study of the deviations of judgements under uncertainty from a normative model but rather the study of the psychological mechanisms underlying such judgements (Kahneman, Slovic \& Tversky, 1982). Kahneman and Tversky's early work (1972, as cited in Tversky \& Kahneman, 1982a, p. 156; see also Kahneman \& Tversky, 1972/1982c; Kahneman \& Tversky, 1973; Tversky \& Kahneman, 1974/1982b) indeed initiated a whole curriculum of research, which became known as the heuristics and biases research programme. They demonstrated that judgements of probability were largely determined by three simple heuristics, namely, the availability, anchoring and representativeness heuristics. The representativeness heuristic, in particular, was proposed as a descriptor of the cognitive mechanisms underlying posterior probability judgements. Although Tversky and Kahneman, (1974/1982b) acknowledged that such heuristics represented an economical short cut to normative strategies, they also warned that the same heuristics could lead to severe and systematic biases when the factors governing uncertainty differed from those determining heuristic judgements.

Kahneman and Tversky's work led to a shift of paradigm (Goldstein \& Hogarth, 1997). Experiments using the book-bag-and-poker-chips paradigm gradually became scarcer. Subsequent studies of posterior probability judgements were carried out under each of two paradigms, namely the Textbook-problem paradigm and the Socialjudgement paradigm (Bar-Hillel, 1983), both instigated by Kahneman and Tversky's earlier studies (e.g., Kahneman \& Tversky, 1973; Kahneman \& Tversky, 1972, as cited in Tversky \& Kahneman, 1982a). The computational accounts of posterior probability judgements in these paradigms will be critically reviewed in the following sections of this chapter, alongside algorithmic accounts characterising the cognitive processes 
underlying those judgements. In particular, arising from the process-focused approach favoured in the present thesis, the main objectives of the review will be (i) to assess the adequacy of the computational accounts of the judgements observed within the heuristics and biases framework (i.e., authors' description of what individuals did to arrive at their judgement), and (ii) the appropriateness of the algorithmic accounts of those judgements (i.e., authors' explanations of how individuals arrived at their judgements).

\section{THE SOCIAL-JUDGEMENT PARADIGM}

\section{Presentation of the paradigm}

The "Social-judgement paradigm" (Bar-Hillel, 1983) was instigated by Kahneman and Tversky's (1973) seminal work. In earlier book-bags-and-poker-chips experiments, the likelihoods of the data under the hypothesis tested and under its alternative were clearly defined by the relative frequencies of coloured chips in the bags. In contrast to those early experiments, Kahneman and Tversky's (1973) original study included social scenarios and likelihoods expressed with thumbnail verbal descriptions rather than numerical information. Typically, Social-judgement tasks involve the assessment of the posterior probability that an instance belongs to a target category, based on both a short verbal description of the instance's social attributes and the base rate of the target category. Base rates may either be provided or elicited from the participants, but the numerical likelihoods of the descriptions are neither presented nor elicited from the participants.

The first study conducted within this paradigm is Kahneman and Tversky's (1973) “Tom W." study. In this study, the authors asked a group of participants to estimate the base rates of American students enrolled in each of nine fields of specialisation, ranging from Business Administration to Social Sciences and Social Work. A second group was given a personality sketch describing a graduate student named "Tom W." The description read as follows:

Tom W. is of high intelligence, although lacking in true creativity. He has a need for order and clarity, and for neat and tidy systems in which every detail finds its appropriate place. His writing is rather dull and mechanical, occasionally enlivened by somewhat corny puns and by flashes of imagination of the sci-fi type. He has a strong drive for competence. He seems to have little feel and little sympathy for other people and does not enjoy interacting with others. Self-centered, he nonetheless has a deep moral sense. (Kahneman \& Tversky, 1973, p. 238) 
Participants in the second group were then asked to rank the same nine fields as those presented to participants in the first group. This time, however, those ranking were made with regards to the extent to which the student described was similar to the typical student in each of those fields. Finally, in a third group, the authors collected rankings for the same nine fields, but instructed participants to rank the descriptions according to the posterior probability that Tom W. had graduated in each of those fields, given his personality description.

In the Tom W. study, all judgements were based on a subjective evaluation of prior probabilities, likelihoods and posterior probabilities. In another, most influential task, within the Social-judgement paradigm, 'objective' base-rates were provided to the participants. An exemplar of this task is the 'lawyer-engineer problem' (Kahneman \& Tversky, 1973). In this problem, the authors manipulated the distribution of instances between two categories- "lawyers" and "engineers"-and tested the effect of such a manipulation on participants' subjective evaluations of posterior probabilities. A first group of participants ('low-engineer' or ' $L$ group') read the following experimental scenario:

A panel of psychologists have interviewed and administrated personality tests to 30 engineers and 70 lawyers, all successful in their respective fields. On the basis of this information thumbnail descriptions of the 30 engineers and 70 lawyers have been written. You will find on your forms five descriptions, chosen at random from the 100 available descriptions. For each description, please indicate your probability that the person described is an engineer, on a scale from 0 to 100.

The same task has been performed by a panel of experts, who were highly accurate in assigning probabilities to the various descriptions. You will be paid a bonus to the extent that your estimates come close to those of the expert panel. (p. 241)

A second group ('the high-engineer' or 'H group') read the same scenario except that the population was described as including 70 engineers and 30 lawyers. Participants were then presented with the same five specific descriptions of individuals. For instance, one of the descriptions read as follows:

Jack is a 45-year-old man. He is married and has four children. He is generally conservative, careful and ambitious. He shows no interest in political and social issues and spends most of his free time on his many hobbies which include home carpentry, sailing and mathematical puzzles. (ibid.)

In both the $L$ and $H$ groups, following each description presentation, the participants were either asked to estimate the posterior probability (in \%) that the person described was one of the 30 (70) engineers or the posterior probability that he was one of the 70 (30) lawyers in the sample of 100 . In other words, crossed with the manipulation of 
base-rates, was the manipulation of the target category resulting in half the participants estimating $p$ (engineer $\mid$ description) and the other half, $p$ (lawyer $\mid$ description).

\section{Computational accounts of judgment outcomes}

\section{Base-rate neglect}

In the original Tom W. study, Kahneman and Tversky (1973) found that posterior probability and similarity rankings were strongly correlated, whereas posterior probability rankings were unrelated to the estimated base rates. The authors interpreted these results as reflecting individuals' tendency to neglect base-rate information. This effect became known as the base-rate neglect effect, or 'base-rate fallacy' (e.g., Bar-Hillel, 1980; Koehler, 1996; Tversky \& Kahneman, 1980).

In the lawyer-engineer study, for any value of the posterior probability that an individual is an engineer $(H)$ given his thumbnail description $(D)$ in the high base-rate condition [i.e., $p(H \mid D)_{\text {High }}$ ], the value of the corresponding posterior probability in the low base-rate condition [i.e., $p(H \mid D)_{\text {Low }}$ ] is normatively defined by Equation III. 1 (The formal demonstration of these relationships is presented in Appendix A, p. 259.)

$$
p(H \mid D)_{\text {Low }}=\frac{p(H \mid D)_{\mathrm{High}}}{k^{2}+p(H \mid D)_{\mathrm{High}}\left(1-k^{2}\right)}
$$

where,

$$
k^{2}=\left(\frac{p(H)_{\text {High }}}{p(H)_{\text {Low }}}\right)^{2}=\left(\frac{.70}{.30}\right)^{2}=5.44 .
$$

Similarly, for any value of $p(H \mid D)_{\text {Low }}$ in the low base-rates condition, the value of $p(H \mid D)_{\text {High }}$ in the high base-rates condition is normatively defined by Equation III. 3,

$$
p(H \mid D)_{\mathrm{High}}=\frac{k^{2} \cdot p(H \mid D)_{\mathrm{Low}}}{1+p(H \mid D)_{\mathrm{Low}}\left(k^{2}-1\right)} .
$$

Figure III-1 presents the graph for Equation III-1. The dots represent the median estimates observed by Kahneman and Tversky (1973), for each of the five descriptions utilised. The authors' main finding was the relatively small effect of the base-rates manipulation on judgements relative to normative Bayesian prescriptions. They indeed observed that, for each of the five specific descriptions presented, the median estimate of $p(H \mid D)_{\text {High }}$ was almost identical to that of $p(H \mid D)_{\text {Low }}$ as indicated by their falling either close to or exactly on the identity line in Figure III-1. Yet Bayes's theorem prescribes that, all other things being equal, judgements in the low base-rate condition should have 
a lower magnitude than those in the high base-rate condition, as indicated by the Bayesian curve in Figure III-1.

Although Kahneman and Tversky (1973) found an overall significant, albeit small, effect of the base-rate manipulation - the average posterior probability judgement was $50 \%$ for the $L$ group, vs. $55 \%$ for the $H$ group, $p<.01$-these results were posited as confirming the proposition that people neglect base-rate information when making a posterior probability judgement.

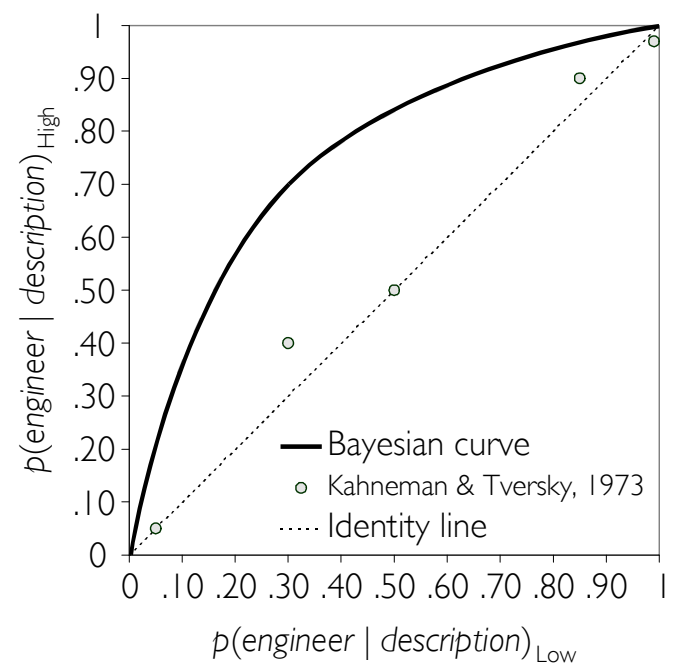

Figure III-1 - Normative relation between low-engineer and high-engineer posterior probabilities for $k^{2}=5.44$ (adapted from Kahneman \& Tversky, 1973).

Note. The dots represent the median judgements observed by Kahneman and Tversky (1973) in the low-engineer condition, plotted against those observed in the high-engineer condition, for each of the five descriptions used.

Following Kahneman and Tversky's (1973) pioneering study, many authors attempted to replicate the original findings observed. Wells and Harvey (1978), for instance, criticised Kahneman and Tversky's (1973) use of medians to test the effect of base-rates for being "underrepresentative of their subjects' behavior" (Wells \& Harvey, 1978, p. 483) because it precluded the analysis of the extent of base-rate utilisation. Wells and Harvey (1978) proposed that a full analysis of the data should involve the comparison, for each description, of all estimates observed in a high base rate condition with all the estimates observed in the low base rate condition. They performed such a comparison and concluded from their results that participants' neglect of base rate information was not as marked as originally claimed by Kahneman and Tversky (1973). Yet a similar analysis comparing judgements with their respective Bayesian standards 
revealed that estimates still significantly differed from those standards (Wells \& Harvey, 1978).

Subsequent Social-judgement studies of posterior probability judgements modelled on the lawyer-engineer problem examined the factors that may induce increased reliance on base-rate information, with little attention paid to the identification of the judgement methods used by individuals. Among those factors feature the causality of base-rate information (Ajzen, 1977), reduced sample sizes, the complete sampling of the descriptions, the possibility of "translating" base rates into whole partitions of the sample (Carroll \& Siegler, 1977), visual random sampling and content familiarity (Gigerenzer et al., 1988), perceived relevance of likelihood and base-rate information (Ginossar \& Trope, 1980), information source characteristics (Ginossar \& Trope, 1987; Schwarz, Strack, Hilton \& Naderer, 1991; Zukier \& Pepitone, 1984), order of information presentation (Krosnick, Li, \& Lehman, 1990), experimental design (Schwarz et al., 1991), diagnosticity (Fischhoff \& Bar-Hillel, 1984), and additivity constraint (Baratgin $\&$ Noveck, 2000).

All above-mentioned studies were based on the lawyer-engineer problem or some isomorphic versions of this problem and entailed the inference of the posterior probability that an individual belonged to a category, given base-rate information about category membership and verbal diagnostic information. The lawyer-engineer task, however, was also adapted to investigate judgements of likely future behaviours and causal attributions. Ajzen and Fishbein (1975; see also Fischhoff \& Lichtenstein, 1978; Ajzen \& Fishbein, 1978) proposed that Bayes's theorem was a potential (normative) model of the attribution process where the posterior probability of a cause, given the observation of a particular behaviour, is a measure of attribution. Nisbett and Borgida (1975) found that the neglect of base rates of category membership information could be generalised to causal attributions. The authors proposed that base-rate neglect explained individuals' tendency to ignore behavioural base rates (e.g., base-rates of 'willingness to take electric shocks', or of 'willingness to help someone apparently suffering a seizure') when attributing causes of behaviours on the basis of target case information. Again, subsequent studies on causal attribution investigated the factors that may affect reliance on behavioural base rates to make causal attributions (for a review, see Borgida \& Brekke, 1981).

Finally, the study of social probabilistic inferences permeated the field of stereotyping studies. McCauley and Stitt (1978) evaluated correlations between posterior probability 
judgements that individuals possessed a personality trait, given their nationality on the one hand, and prior probability judgements that individuals possessed a personality trait, on the other hand. Although the authors found no evidence for base-rate neglect, they found some evidence indicating that the additivity principle was not always respected. Locksley, Borgida, Brekke, and Hepburn (1980) investigated the effect of stereotypes on posterior probability judgements of $p$ (trait|behaviour) and concluded that, in the presence of diagnostic information, stereotypical base rates of personality traits-for example, the perceived probability that a male is assertive-were ignored. Locksley, Hepburn and Ortiz (1982) replicated this result. They also compared derived normative estimates based on Bayesian prescriptions with actual judgements, and found that the latter significantly differed from their normative counterparts. Subsequent studies investigated the factors affecting reliance on stereotypic beliefs. Among those factors featured the salience of base-rate information (Beckett \& Park, 1995), the diagnosticity of stereotypical category and the level of consistency and desirability of the observed behaviour in predicting the target personality (Krueger \& Rothbart, 1988), and the diagnosticity of the behaviour for predicting the personality trait (Krueger \& Rothbart, 1988; Rasinski, Crocker, \& Hastie, 1985).

In sum, a common characteristic of the computational accounts arising from Socialjudgement studies is the focus on the neglect of base-rate information and the examination of the factors that may enhance its utilisation. Such a line of investigation illustrates the dominance of the aforementioned outcome-focused approach to the study of posterior probability judgements. Under this approach, researchers aim at both examining the reasons behind the lack of judgements' accuracy and identifying the external conditions or appropriate aiding tools that would improve the accuracy of those judgements. By contrast, from the process-focused approach favoured in this thesis, a primary objective is to provide an appropriate computational account of individuals' posterior probability judgements; that is, to achieve a description of what individuals did to arrive at their judgements.

The different contexts within which judgements were gathered and the different types of measures collected suggest that the evidence available within the Socialjudgement paradigm may only superficially inform a computational account of social judgements of posterior probability. Results within this paradigm were indeed based on a variety of measurement methods of posterior probabilities, ranging from rank-ordering methods (e.g., Kahneman \& Tversky, 1973) to the collection of probability estimates 
(e.g., Ajzen, 1977; Kahneman \& Tversky, 1973; Schwarz et al., 1991; Wells \& Harvey, 1978) or likelihood estimates (e.g., Krosnick, Li, \& Lehman, 1990), absolute numbers (e.g., Carroll \& Siegler, 1977; Nisbett \& Borgida, 1975), chance estimates (e.g., Baratgin \& Noveck, 2000; Ginossar \& Trope, 1987), percentage of individuals (Locksley et al., 1980; Locksley et al., 1982; McCauley \& Stitt, 1978; Rasinski, Crocker, \& Hastie, 1985), and percentage of time estimates (e.g., Krueger \& Rothbart, 1988). Despite this variety, analogous analyses were carried on the data collected across experiments. Namely posterior probability judgements' mean estimates for high base-rates were compared with corresponding mean estimates for low base-rates. In other words, the extent to which individuals neglect base rates has been consistently measured by the extent to which $p(H \mid D)_{\text {High }}$ differs from $p(H \mid D)_{\text {Low }}$.

Yet one may ask to what extent the absence of a difference between the mean outputs observed under high and low base-rate conditions, respectively, indicates the extent to which individuals actually neglect base-rates. Authors (e.g., Rapoport \& Wallsten, 1972; Slovic, \& Lichtenstein, 1971) had already warned against the assimilation of models describing mean outputs of posterior probabilities with the psychological processes underlying those judgements, in conservatism studies (e.g., Phillips \& Edwards, 1966). The evidence available within the Social-judgement paradigm is at best consistent with the proposition that the majority of individuals neglect base-rates when making posterior probability judgements but it cannot conclusively demonstrate that most individuals actually do neglect base rates. Besides, from a processfocused approach, one may question the validity of the base-rate neglect computational account of posterior probability judgements. Even if the base-rate neglect account of mean outputs consisted in an accurate description of individuals' behaviour, it would only inform what individuals do not do (i.e., they do not integrate base rates in their judgements), with no indication as to what they may do instead.

Therefore, the process-focused stance highlights a first issue to be addressed, namely whether the base-rate neglect is a useful computational account of the judgements observed and whether there are better candidates for describing individuals' judgement methods underlying the judgement outputs observed. It is possible, for example, that people evaluate the posterior probability $p(H \mid D)$ on the basis of its corresponding inverse likelihood $p(D \mid H)$. This method of judgement, called the inverse fallacy (Koehler, 1996), has mostly been invoked to explain the origin of observed outputs within the Textbook-problem paradigm (see p. 56). Some authors have suggested, 
however, that the same method of judgement is applied in Social-judgement tasks (Gigerenzer \& Murray, 1987; Pollard \& Evans, 1983; see also Lagnado \& Shanks, 2002). Within the Social-judgement paradigm, the inverse fallacy account would suggest that people evaluate the posterior probability p(category|description) that an instance belongs to a category given its description, on the basis of the inverse likelihood $p$ (description|category) of observing the same description, in the knowledge that the instance belongs to the target category. Such account, however, remains to be empirically validated.

\section{Additivity}

In the seminal lawyer-engineer study, Kahneman and Tversky (1973) concluded that the manipulation of the target hypothesis had no effect since the sum of median probabilities assigned to $p$ (engineer $\mid$ description) and $p$ (lawyer $\mid$ description) approached $100 \%$ for each description. Consequently, assuming that participants respected the additivity principle (see Chapter II, p. 16), the authors recoded estimates of $p$ (lawyer $\mid$ description) as estimates of $p$ (engineer|description) by subtracting the former from 100, and pooled the data together. As noted by Baratgin and Noveck (2000), subsequent Social-judgement studies of posterior probabilities for the most part assumed additivity and did not collect posterior probability judgements related to the alternative hypothesis.

Yet, as early as 1954, Edwards had questioned whether we should assume additivity of complementary judgements. He reported findings by Kaplan and Radner showing that the sums of probability judgements assigned to sets of exhaustive and mutually exclusive statements increased with the number of alternatives. Teigen (1974) conducted two experiments specifically designed to assess the additivity of complementary probability judgements. The results he observed confirmed that judgements deviated from additivity, and such deviations were related to the number of alternatives involved in the assessment. Since these early investigations, others have examined additivity of probability judgements and achieved similar conclusions (Caroll \& Siegler, 1977; Gigerenzer et al., 1988; Schwarz et al., 1991; Wells \& Harvey, 1978). Yet subsequent Social-judgement studies of posterior probabilities, for the most part, assumed additivity and did not collect posterior probability judgements related to the alternative hypothesis (Baratgin \& Noveck, 2000). 
Baratgin \& Noveck (2000) reported that even when participants were induced to produce additive posterior probability estimates by being required to assess both $p$ (lawyer|description) and $p$ (engineer|description), about 20\% of the participants still violated the additivity assumption. This result confirms that the extent to which the additivity assumption provides an accurate description of individuals' complementary posterior probability estimates is contentious. Moreover, the description of judgement processes in terms of base-rate neglect precludes the prediction of individuals' deviations from additivity. It is only by describing what individuals indeed do when making posterior probability judgements that it will be possible to predict whether or not those individuals will respect the additivity principle.

From a more general, descriptive standpoint, there is also disagreement within the literature as to whether we should expect to observe judgements of two complementary probabilities to be additive. Some researchers (e.g., Tversky \& Koehler, 1994; Rottenstreich \& Tversky, 1997) reported that binary complementary judgements were additive; that is, that judgements of the probabilities $p(H)$ and $p(\neg H)$, where $\neg$ represents the explicit negation of hypothesis $H$ would be equal to one. By contrast, other researchers (e.g., Ayton, 1997) reported that people's complementary probability judgements tend to be subadditive (i.e., the sum of the individual complementary estimates is greater ${ }^{1}$ than one). In addition, evidence of superadditivity (i.e., estimates summing to less than one) in the case of binary complementarity has also been found (e.g., Macchi, Osherson, \& Krantz, 1999).

Within the specific context of posterior probability judgements, a computational account would provide a better ground for addressing the additivity issue. For instance, if people commit the inverse fallacy when making their posterior probability judgements, then deviations from additivity of the sum of judgements of complementary posterior probabilities $p(H \mid D)$ and $p(\sim H \mid D)$ should be predictable from the sum of their corresponding inverse probabilities, $p(D \mid H)$ and $p(D \mid \sim H)$.

So far, the issues identified were related to the computational account of judgements observed within the Social-judgement paradigm. In the remainder of this section, the existing algorithmic accounts of the cognitive processes underlying posterior probability judgements within this paradigm are appraised.

\footnotetext{
'Although the terminology used might seem counterintuitive, the meanings of 'subadditive' and 'superadditive' judgements are defined to be consistent with the terminology used in previous studies on unconditional probability judgements (e.g., see Tversky \& Koehler, 1994, p. 553) where the sum of complementary judgements was characterised as subadditive when it exceeded 1 .
} 


\section{Algorithmic accounts of underlying reasoning processes}

\section{The representativeness heuristic}

Kahneman and Tversky (1973) similarly interpreted both results observed in the Tom W. study and those observed in the lawyer-engineer problem. Namely, the authors proposed that participants evaluate their posterior probability judgements that an instance belonged to a category given its description on the extent to which the description is representative of a typical instance of this category, thus giving little consideration to the base rates of category membership. They called this process the representativeness heuristic.

Tversky and Kahneman (1983) further defined this heuristic as:

an assessment of the degree of correspondence between a sample and a population, an instance and a category, an act and an actor or, more generally, between an outcome and a model. The model may refer to a person, a coin, or the world economy, and the respective outcomes could be marital status, a sequence of heads and tails, or the current price of gold. (p. 295)

The authors also specified that representativeness was directional: one can assess the extent to which an outcome is representative of a model, but not whether a model is representative of an outcome. Judgements of representativeness may correspond to judgements of similarity (e.g., in assessing the extent to which a sample is similar to a given population or the extent to which an instance is similar to the typical or prototypical member of a given category). Nevertheless, representativeness cannot be reduced to similarity, it can also rely on judgements of propensity (e.g., a given act can be judged to be representative of an actor based on the extent to which the actor is predisposed to commit this act). Representativeness tends to covary with frequency, yet it is not determined by it: representativeness can be at odds with actual frequency (e.g., specific outcomes can be representative although infrequent) or perceived frequency (e.g., diagnostic outcomes can be representative yet perceived as infrequent). Finally, representativeness is not bounded by class inclusion: an instance can be unrepresentative of a category yet highly representative of a superordinate category (Tversky \& Kahneman, 1983; see also Tversky \& Kahneman, 1982c).

There are at least two main issues regarding the representativeness heuristic. The first issue concerns its definition: authors have criticised its lack of formalism (e.g., Wallsten, 1983; Gigerenzer \& Hoffrage, 1995) and its post-hoc explanatory nature. Groner, Groner, and Bischof (1983; see also Gigerenzer, 1996; Wallsten, 1980), for instance, contended, 
representativeness as an explanatory concept suffers from a serious difficulty: it is much easier to state a posteriori that some evidence must have been representative for a certain outcome, than to make a prediction for which outcome an evidence will be representative. (p. 104)

Wallsten (1980) noted the existence of alternative explanations, such as the effect of the saliency of "vivid" individuating information (Nisbett, Borgida, Crandall, \& Reed, 1976/1982), which have similar post-hoc explanatory power. The second issue related to the representativeness heuristic concerns whether it actually models how individuals make posterior probability judgements (algorithmic level of analysis) or whether it is just another illustration of what this process may entail, i.e., reliance on considerations of representativeness (computational level of analysis). In their original paper, Kahneman and Tversky (1973) contended that the first alternative held: "Intuitive predictions follow a judgmental heuristic_-representativeness.” (p. 237). Nevertheless, about a decade later, the authors revised their statement in favour of the second alternative, positing the representativeness heuristic as one rule among others, which people may follow in estimating posterior probability judgements (Tversky \& Kahneman, 1982c) Still, yet another decade later, Kahneman and Tversky (1996) recognised that despite their attempts, they "were not able to offer a comprehensive treatment of the process by which different representations and different tasks evoke different heuristics" (Kahneman \& Tversky, 1996, p. 583). Therefore, throughout the years, the status of the representativeness heuristic as an explanation for the cognitive processes underlying posterior probability judgements has been increasingly eroded. In fact, the representativeness heuristic may explain what individuals do when making their judgements, but not how they do so. It is thus an alternative computational descriptor of the behaviours occurring within posterior probability judgement tasks; it is not an algorithmic account of these behaviours.

\section{Problem representation and cognitive strategies}

Gigerenzer et al. (1988) proposed that posterior probability judgements originated from participants' "internal problem representation" (p. 513). Such problem representation, Gigerenzer et al. (1988) argued, is itself mediated by both the way in which information is presented (e.g., a visual presentation of sampling procedures induces a statistical representation of the task) and participants' familiarity or previous experience with the problem content. Figure III-2 reproduces their schema of human probability revision. 


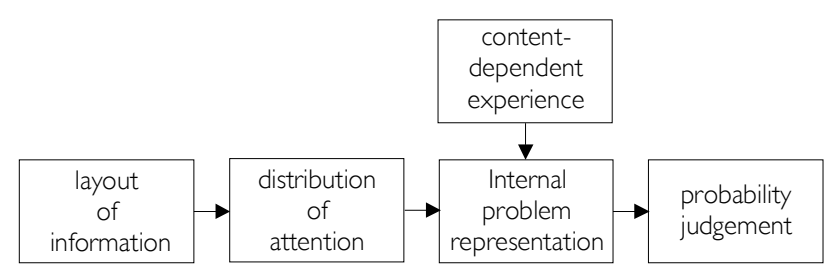

Figure III-2 - Gigerenzer et al.'s (1988) schema of human probability revision

Although Gigerenzer et al.'s (1988) schema is closer to a cognitive model of posterior probability judgements than the representativeness account, it is somewhat restrictive as it stands. For instance, according to Zukier and Pepitone (1984), it is the orientation of the task that determines how attention is distributed and how the task is represented. Such a concept of orientation encompasses both information layout (context) and information framing (e.g., statistical vs. clinical framing). Yet this latter element is lacking from Gigerenzer et al.'s (1988) schematic account.

Zukier and Pepitone (1984) also suggested that different orientations might entail the application of different cognitive strategies. Gigerenzer et al. (1988) omitted such inferential aspects in their model, which suggests that probability judgements are directly inferred from the task representation. Paradoxically, the authors' own data on reported strategies confirmed Zukier and Pepitone's (1984) suggestion. When participants in Gigerenzer et al.'s study were asked to describe as precisely as possible the process by which they arrived at their probability judgements in the standard lawyer-engineer problem, $68 \%$ reported that their strategy had been based on similarity considerations. This percentage remained high (55\%) when base rates were presented visually (i.e., when random sampling was performed and observed by the subjects themselves). The examination of those strategies also revealed evidence for the use of base-rate information. This outcome, however, might have resulted from participants' post-hoc rationalisation induced by the authors' question: "When (under what condition) did you consider the base rates of engineers and lawyers?" (p. 515). Wason and Evans (1975), for instance, observed such "normative" rationalisations with the selection task (Wason, 1966) from participants whose preceding selections were not in line with normative standards. In any case, Gigerenzer et al.'s (1988) results suggested that representativeness is not the only information processing strategy elicited and that different tasks may elicit different pre-existing strategies, a process that is not explicitly accounted for in the authors' schematic model of probabilistic inference. 
In contrast to the Gigerenzer et al. (1988) model, but in line with Zukier and Pepitone's (1984) argument, Ginossar and Trope (1987) proposed that the representation of the task is one of the factors influencing the type of inferential rules elicited. Specifically, the authors proposed that the representation would lead either to the application of the representativeness heuristic or to the application of an intuitive sampling rule'. The intuitive sampling rule entails the assessment of the probability of an event on the basis of "its relative frequency in the population from which it was drawn" (Ginossar \& Trope, 1980, p. 231). Ginossar and Trope (1987) also established that other factors such as the prior activation of the rule and its conditions of application may influence the rule activated and consequently the judgement outcomes observed. Nevertheless, although these authors' theoretical framework provides a more promising approach to the study of internal processes underlying posterior probability judgements, its application to posterior probability judgements leaves unanswered questions.

Ginossar and Trope's (1987) results—based on an analysis of mean outputs-do not permit one to infer the extent to which each individual participant relied on the representativeness heuristic, the intuitive sampling rule or yet another strategy in making their judgements. Ginossar and Trope (1980) suggested that the application of a rule might also depend on its availability in long-term memory. But, neither their results nor those reported in Ginossar and Trope (1987) reliably account for these individual differences. Also, although there is some evidence that people rely on the similarity between the individuating descriptions and the typical representation of, say, an engineer (Gigerenzer et al., 1988), there is no direct evidence that the application of the intuitive sampling rule actually results in the consideration of base-rate information and vice-versa. Finally, the accounts presented above neither specified what the representation of the task may entail, nor the exact modalities by which a particular representation will elicit a particular rule.

\section{Conclusions}

The process-focused review of posterior probability judgements within the Socialjudgement paradigm has identified several shortcomings of the present state of knowledge. From a computational perspective, it was argued that the evidence available does not allow the distinction of "as-if" (Lopes, 1981) descriptions of aggregated judgements from an actual description of individuals' behaviours. The validity of the base-rate neglect computational account was also questioned as it describes what 
individuals may not do. The inverse fallacy was suggested as a better descriptor, although its validity within this paradigm has yet to be demonstrated empirically. In any case, an adequate computational account of behaviours observed in posterior probability judgement tasks would also allow the prediction of the extent to which complementary estimates would be additive. Specifically, the explicit knowledge of the processes by which individuals make posterior probability judgements would allow the prediction of the direction and the magnitude of complementary judgements' deviation from unity.

The review of existing accounts of posterior probability judgements in the Socialjudgement paradigm revealed that the explanatory status of the representativeness heuristic was debatable: it describes what individuals may do when making their posterior probability judgements (i.e., rely on assessments of representativeness), yet it does not satisfactorily explain how they may do so. As such, it becomes an alternative computational account of posterior probability judgements within the Social-judgement paradigm. Therefore, the representativeness heuristic also must be empirically distinguished from the former two computational accounts, namely, the base-rate neglect account and the inverse fallacy account.

The remaining accounts of the origin of posterior probability judgements in Socialjudgement tasks proposed that the type of interpretation of the task, determined by the layout of the statistical information or the framing of the task influenced its mental representation. Such mental representation was said to determine the output of the judgement, if not the strategy applied to make such a judgement. Although this is a compelling explanation for the origin of the judgements observed, empirical tests of these accounts were based on aggregated judgements. It is therefore impossible to determine whether individuals actually relied on either one of the two strategies proposed (the representativeness heuristic and a sampling rule) or even any other strategy. It is also impossible to determine whether what is inferred to be an increased reliance on base-rates, actually results from the application of a frequentistic rule. Finally, although these accounts emphasized the role of the representation of the task, they neither included a formal description of such a representation nor specifically defined how the features of the task would mediate the application of a specific strategy.

In the next section of this chapter, the research carried out within the Textbookproblem paradigm is reviewed. The same guiding principles as those observed for the review of the Social-judgement paradigm are followed. Namely, after describing this latter paradigm, the review aims at assessing (i) the existing computational accounts of 
posterior probability judgements within this paradigm, and (ii) the appropriateness of existing algorithmic accounts for those judgements.

\section{THE TEXTBOOK-PROBLEM PARADIGM}

\section{Presentation of the paradigm}

In the same way studies carried out within the Social-judgement paradigm were instigated by Kahneman and Tversky's (1973) original work, studies carried out within the "Textbook-problem paradigm" (Bar-Hillel, 1983) were also initiated by a seminal task, namely the Taxicab problem. This task was first reported in the Oregon Research Institute Bulletin (1972, as cited in Tversky and Kahneman, 1982a) and later further investigated by Bar-Hillel (1980) and Tversky and Kahneman (1980).

The cab problem (Kahneman \& Tversky, 1972, as reported in Tversky \& Kahneman, 1980) read as follows:

A cab was involved in a hit-and-run accident at night. Two cab companies, the Green and the Blue, operate in the city. You are given the following data:

(i) $85 \%$ of the cabs in the city are Green, and $15 \%$ are Blue.

(ii) A witness identified the cab as a Blue cab. The court tested his ability to identify cabs under the appropriate visibility conditions. When presented with a sample of cabs (half of which were Blue and half of which were Green) the witness made correct identifications in $80 \%$ of the cases and erred in $20 \%$ of the cases.

What is the probability that the cab involved in this accident was Blue rather than Green? (p. 290)

More generally, research within the Textbook-problem paradigm entails the administration of tasks requiring participants to estimate posterior probabilities on the basis of numerical values for the base rates $p(H)$ and $p(\sim H)$, and-in contrast with the Social-judgement paradigm—numerical values for the likelihood of observing the data $D$, given that $H$ is known to be true, $p(D \mid H)$, and the likelihood of the data when $H$ is known to be false, $p(D \mid \sim H)$. The quantification of all probability information required to infer the posterior probability thus enables the comparison of participants' judgements with normative values prescribed by Bayes's theorem.

\section{Computational accounts of judgment outcomes}

\section{Base-rate neglect}

The cab problem features two complementary hypotheses. Hypothesis $H$ is "the cab involved in the accident was Blue," and the alternative hypothesis $\sim H$ is "the cab involved was Green". The data $D$ is "a witness identified the cab as Blue." The values of 
the base rates for $H$ and $\sim H$ are $15 \%$ and $85 \%$, respectively. And the likelihood that a Blue cab is indeed identified as a Blue cab, $p(D \mid H)$ is $80 \%$ whereas the likelihood that a Green cab is identified as a Blue one, $p(D \mid \sim H)$, is 20\%. Consequently, according to Bayes's theorem, the normative answer to this problem is given by

$$
p(H \mid D)=\frac{.80 \times .15}{.20 \times .85+.80 \times .15}=.41
$$

Typically, however, the modal value of the posterior probability judgement provided by participants is 80\%; that is, the value of the likelihood $p(D \mid H)$ (Bar-Hillel, 1980; Tversky \& Kahneman, 1980). Authors interpreted these results as demonstrating the base-rate fallacy; that is, the tendency to either ignore or else grossly underweight baserate information (e.g., Bar-Hillel, 1980; Lyon \& Slovic, 1976; Tversky \& Kahneman, 1982a). The so-called 'base-rate fallacy' was thereafter observed with different scenario contents (Hammerton, 1973; Lyon \& Slovic, 1976), different likelihood values (Liu, 1975), different base-rate values, different base-rate salience, different orders of presentations of base-rate and likelihood information (Lyon \& Slovic, 1976). This tendency was also observed in Medical doctors' judgements (Eddy, 1982).

Following the establishment of the base-rate fallacy within the Textbook-problem paradigm, research within this paradigm followed the exact same path as research within the Social-judgement paradigm. Thus, subsequent Textbook-problem studies, for the most part, examined the factors that may mediate base-rate usage, with little attention paid to the judgement methods underlying observed judgement outputs. For instance, base-rate usage was shown to be affected by the causality of base-rate information (Tversky \& Kahneman, 1980), the numerical values of base rates and likelihood information (Birnbaum \& Mellers, 1983; Hinsz, Tindale, Nagao, Davis, \& Robertson, 1988; Lynch \& Ofir, 1989; Ofir \& Lynch, 1984), experimental design features such as within-subjects variation (Birnbaum \& Mellers, 1983; Fischhoff, Slovic, \& Lichtenstein, 1979; Ofir \& Lynch, 1984), relative specificity of base-rate and likelihood information (Bar-Hillel, 1980; Lynch \& Ofir, 1989; Ofir \& Lynch, 1984), direct experience of baserates (Christensen-Szalanski \& Beach, 1982), problem verbal structure (Macchi, 1995), statistical training (Wolfe, 1995); cognitive correlates (Stanovich \& West, 1998).

Thus, as in the Social-judgement paradigm, computational accounts of posterior probability judgements within the Textbook-problem paradigm are based on the baserate neglect account. Again, from the process-focused approach favoured here, the crucial issue to be addressed is whether available evidence provides the basis for 
understanding what individuals did to arrive at their judgements. The descriptive adequacy of the base-rate neglect effect in accounting for posterior probability judgements in Textbook-problem has already been criticised within the literature. Hamm $(1993,1994)$ indeed argued that whereas "neglect" points toward a lack of attention to the base-rate information, the judgements observed in the cab problem and problem isomorphs reflect individuals' confusion between $p(D \mid H)$ and $p(H \mid D)$-later designated the inverse fallacy by Koehler (1996) — rather than base-rate neglect ${ }^{2}$. Wolfe (1995) further illustrated the prevalence of the inverse fallacy in an investigation of individuals' interpretations of $p(D \mid H)$ and $p(H \mid D)$ concepts and provided evidence that the inverse fallacy may mediate base-rate neglect. The author indeed experimentally demonstrated that participants who were trained to distinguish $p(D \mid H)$ from $p(H \mid D)$ were more likely to seek base-rate information compared with a control group (Wolfe, 1995, Experiment 3). Yet that the inverse fallacy provides a better computational account of posterior probability judgements than the base-rate fallacy remains to be empirically tested.

Within the Social-judgement paradigm, the extent to which base rates were neglected was inferred from the comparison of mean posterior probability judgements in high and low base-rate conditions. As it was contended earlier in this thesis, such evidence may not inform the psychological processes underlying individuals' judgements. Within the Textbook-problem paradigm, in contrast, the presence of numerical likelihood information allows for the identification of the proportion of judgements that match the Bayesian value of the posterior probability $p(H \mid D)$ or, for instance, the value of its corresponding inverse probability $p(D \mid H)$.

Within the Textbook paradigm, the extent of 'base-rate neglect' has traditionally been evaluated in terms of the proportion of judgements matching the inverse probability $p(D \mid H)$ (e.g., see Tversky \& Kahneman, 1980; Bar-Hillel, 1980). Individual variability has been reported through the presentation of the distribution of the judgements observed (e.g., Bar-Hillel, 1980) or the estimation of individual cueutilisation policies (Ofir \& Lynch, 1984). Although the majority of observed judgements remained unaccounted for, such an idiographic approach is more informative than the

\footnotetext{
${ }^{2}$ Although the Textbook-problem paradigm was developed subsequently to the Social-judgement paradigm, the inverse fallacy was first characterised in studies within the Textbook-problem paradigm, and thereafter suggested as a method used by participants in Social-judgement tasks.
} 
average-based approach broadly followed within the Social-judgement paradigm, as it directly illustrates variations in modal outputs.

But, this descriptive approach is not always without shortcomings. For instance, one may ponder on the correspondence between the cue-utilisation statistical weights identified by linear regression models (e.g., see Ofir \& Lynch, 1984) and the extent to which individuals actually rely on these respective pieces of information to form their judgements. The same remark applies to cases where judgements whose value fell between the value of the likelihood, $p(D \mid H)$, and the value of the base rate, $p(H)$, were taken as evidence of an integration of both these pieces of information in the judgement (e.g., see Tversky \& Kahneman, 1980, p. 64). At best, such judgements indicate that judges do not exclusively rely on the value of $p(D \mid H)$, but one can only speculate as to whether those judgements are also the result of a consideration of the base-rate information. For instance, Macchi (1995, p. 192) reported that verbal reasoning protocols revealed that the same numerical answer could result from the combination of base-rate and likelihood information or from an adjustment of the likelihood information independent of the base-rate information. She opted for a triangulated index whereby she relied on both verbal protocols and numerical outputs to classify individual judgements. As a result, she distinguished between responses based on $p(D \mid H)$ information alone from those integrating both $p(D \mid H)$ and $p(H)$. Although results from this methodology are more informative than results based on the sole analysis of numerical outputs, this dichotomy still does not allow the individual judgement methods underlying posterior probability judgements observed in Textbookproblem studies to be specifically identified.

\section{Additivity}

Earlier remarks made concerning the additivity of posterior probability judgements in Social-judgement studies (see p. 47), of course, hold within the Textbook-problem paradigm. The assumption that participants' complementary judgements are additive is also found in studies using Textbook problems (e.g., see Slowiaczek, Klayman, Sherman, $\&$ Skov, 1992). Yet the empirical investigation of the additivity of posterior probability judgements studied within the Textbook-problem paradigm has yet to be conducted. As with Social-judgement studies, a sound computational account of posterior probability judgements produced in Textbook-problems is essential to address the additivity issue. 


\section{Algorithmic accounts of underlying reasoning processes}

\section{The representativeness heuristic}

Kahneman and Tversky (1973) first proposed that posterior probability judgements were generally mediated by representativeness, whereby positing the representativeness heuristic as an explanatory construct for the processes underlying such judgements. At the time, scholars enthusiastically welcomed the authors' heuristics and biases approach to judgements under uncertainty (e.g., Slovic et al., 1977). Earlier studies of conservatism had indeed been criticised for focusing on the deviation between observed outputs and normative values (the conservatism effect) rather than on psychological accounts of the heuristic strategies that underlie judgements (e.g., Rapoport \& Wallsten, 1972; Slovic et al., 1977). Hence, the heuristics and biases approach appeared to be a promising shift towards the study of psychological processes. Yet it was not long before the focus of posterior probability judgement studies once more shifted back to the study of observable judgement outputs, with the only difference that interest in the conservatism effect was supplanted with that in the base-rate neglect effect. Indeed, Tversky and Kahneman (1980; see also Bar-Hillel, 1990) themselves argued that, in Textbook-problems, probability judgements were not mediated by representativeness. In the taxicab problem, for instance, observed posterior probability judgements could hardly be explained as manifesting the evaluation of the degree with which the witness identified the $c a b$ as Blue is representative of a typical Blue $\mathrm{cab}^{3}$. Hence, the authors concluded that the base-rate neglect was "a more general phenomenon" (p. 63) and called for more research into the factors that would mediate the integration of base-rate information in posterior probability judgements.

\section{The causality account}

When the generality of the base-rate effect superseded that of its explanation, Tversky and Kahneman (1980) called for "the specification of the conditions under which base-rate information is neglected" (p. 63). The authors proposed that one such condition was the extent to which base-rate information fitted in a causal schema. For instance, the authors contended that the proportion of judgements solely based on $p(D \mid H)$ information decreased when base-rates in the cab problem referred to cabs in

\footnotetext{
${ }^{3}$ In situations where people have, for instance, some knowledge of the companies' safety records, it is however conceivable that their judgement would rely on considerations of representativeness (D. E. Kornbrot, personal communication, September 2002). Other interpretations include that of the use of the witness's reliability (Manktelow, 1999) or the salience of the witness's testimony (Baron, 1994) as an index of representativeness.
} 
accidents rather than cabs in the city, because the latter formulation readily elicited a causal interpretation. Namely, they argued that people would infer that it was the relative competence of the drivers-reflected in the differential base-rates of accidents - that was at the origin of the witnessed accident, and thereafter integrated this information in their final judgement.

Although this explanation is intuitively compelling and psychologically plausible, it cannot be taken as a definite account of the processes underlying posterior probability judgements. As for the representativeness heuristic, the causality explanation indeed lacks formalism. The features that trigger causal interpretations remain to be specified, yet causal attribution is a complex phenomenon, as merely demonstrated by the extent of the literature devoted to this subject (see, e.g., Hewstone, 1989). So, the causality account remains somewhat superficial since the exact ways in which causality interpretations may determine judgement outcomes were never elaborated upon.

\section{The relevance account}

Bar-Hillel (1980) criticised Tversky and Kahneman's (1980) causality account on yet another ground, namely its lack of generalisability. She argued that causality was one factor among others that may induce information integration, thus accounting for "when rather than why base rates will be ignored" (p. 216). She proposed that attention to base rates was more generally determined by their perceived degree of relevance, in turn mediated by aspects such as causality, but also, for instance, information specificity. BarHillel (1980) indeed proposed that when making a judgement about a member of a population subset, specific information about instances of such subset would be perceived as more relevant than general information about instances in the population at large (see also Lynch \& Ofir, 1989; Ofir \& Lynch, 1984; Hinsz et al., 1988). Still, as with the causality account, the determinants of relevance attribution and the cognitive mechanisms underlying observed judgement outcomes when such attributions are induced by the task, remain to be explicitly defined. Bar-Hillel (1980) herself recognised the importance of such an issue, as well as its wide-ranging scope, extending beyond causality and specificity.

Subsequent studies that corroborated the importance of specificity (e.g., Ofir \& Lynch, 1984) on the basis of the use of statistically-derived linear models of cueutilisation also remain mute with regards to the processes underlying the aggregation of such cues when the posterior probability judgement is formed (e.g., see Selling \& Shank, 
1989). Similarly, attempts to fit processing models to judgements (e.g., Birnbaum, 2001;

Birnbaum \& Mellers, 1983) are subject to the same "as if" criticism mentioned earlier: a good fit to the data does not necessarily mean a good fit with the underlying process that generated the data observed.

\section{The 'partitive' account}

Macchi (1995) argued that neither specificity nor causality could explain the lack of reliance on base-rate information, but rather that base-rate usage was hindered by the 'pragmatic structure' of the problem. Specifically, she proposed that unless the verbal formulation of the likelihood information was 'partitive,' participants would interpret this information "as if it were already the result of its combination with the prior probability" (p. 190) and consequently neglect base-rate information. The author summarised the effect of partitive formulations as follows:

In broader terms, the partitive element transmits the idea that the specific information is related to a property (e.g., the property of being recognized as Blue) defining a sub-set that is not to be considered separately, but as a part of the global set to which the base rate refers. (Macchi, 1995, p. 204).

This formulation, which I call partitive, consists of defining the set of which the datum ... represents a part and then relativizing its numerical content. Thus, " $80 \%$ of the people affected by the disease . . . have the symptom" defines the proportion (or subset) of the population with the disease (i.e., the base-rate probability) and has particular properties. Specifically this wording clarifies the relationships between the probabilities relating to the subsets and facilitates Bayesian reasoning. (Macchi, 2000, p. 219)

Macchi's (1995, 2000) partitive account, however, falls short of providing an explanation for the reasoning processes underlying posterior probability judgements. The partitive account suggests that the verbal structure of Textbook problems influences judgements. Nevertheless, as for the other interpretational accounts reviewed, the exact modalities of such influence remain undisclosed. Macchi's account suggests that the partitive formulation clarifies the relationship between different probabilities in the problem. In particular, Macchi (1995) suggested that the partitive formulation clarifies the independence of $p(H)$ and $p(D \mid H)$. Without such clarification, Macchi argued, individuals tend to interpret $p(D \mid H)$ as already integrating the base-rate information, and therefore confuse $p(H \mid D)$ and $p(D \mid H)$. Yet if people believed that $p(D \mid H)$ already integrated the base-rate information, sensu stricto they would confuse $p(D \mid H)$ and $p(D \cap H)$ rather than $p(D \mid H)$ and $p(H \mid D)$ because $p(D \mid H) \cdot p(H)=p(D \cap H)$. Hence,

it is not clear why the absence of a partitive formulation entails $p(H \mid D)$ judgements to be assimilated with the value of $p(D \mid H)$ rather than with the value of $p(D \cap H)$. 


\section{Conclusions}

In sum, following the process-focused approach favoured in this thesis, the review of posterior probability judgements within the Textbook-paradigm has led to the identification of similar shortcomings to those that were identified within the review of the Social-judgement paradigm. Namely, it was argued that the evidence available suggested that judgements might be better described in terms of the inverse fallacy rather than in terms of base-rate neglect. In contrast with the Social-judgement paradigm where no empirical evidence was available to test this hypothesis, results observed within the Textbook-problem paradigm revealed that judgements often appear to be solely based on the value of $p(D \mid H)$. Yet all but a few authors continue to describe this result as an instance of the base-rate fallacy. A first step towards a more accurate description of posterior probability judgements within this paradigm would thus be to determine empirically the extent to which base-rate neglect and inverse fallacy indeed describe the same fallacy. In addition, the knowledge of what individuals do when making their judgements would in turn allow one to predict the extent to which they would deviate from the additivity principle. In contrast with Social-judgement studies, studies conducted within the Textbook-problem paradigm more often reported idiographic information (e.g., by presenting the distribution of participants' judgements), thereby increasing the descriptive validity of the results observed, with specific regards to a process-focused approach. Yet many judgements remain unaccounted for.

With regards to algorithmic developments, little is known on the processes underlying the integration of the different pieces of statistical information to produce the outcome of judgement when it appears that the base-rate information is integrated. The origin of the 'base-rate neglect' in Textbook problems has been accounted for by interpretational accounts, emphasizing the role of the interpretation of the information provided in the task. Yet those accounts still leave many theoretical questions unanswered. In particular, although interpretational accounts suggest that different types of interpretations would determine which information would be attended to by participants, these accounts do not specify how such influence operates. In other words, it is still unclear why and how interpretational factors affect judgement behaviours.

This review will now turn to the latest paradigm within which posterior probability judgements have been researched, which I called the "Set-based" paradigm. As with the two preceding paradigms, the following guiding principles will be applied: after describing what the Set-based paradigm entails, the review will aim at assessing (i) 
computational accounts of Set-based posterior probability judgements, and (ii) the appropriateness of algorithmic explanations for the processes underlying Set-based judgements.

\section{THE SET-BASED PARADIGM}

\section{Presentation of the paradigm}

The Set-based paradigm for the study of posterior probability judgements is fairly recent and its origin is often attributed to the work by Gigerenzer and Hoffrage (1995) and that by Cosmides and Tooby (1996). Set-based problems are similar to Textbookproblems in that they present numerical information for all the elements of Bayes's theorem [i.e., $p(H), p(\sim H), p(D \mid H)$, and $p(D \mid \sim H)]$ and require participants to make posterior probability judgements on the basis of this information. However, Set-based tasks differ substantially from Textbook problems in the format and the structure of both the information presented and the question asked.

Gigerenzer and Hoffrage (1995) first proposed that Bayesian reasoning could be facilitated by the use of natural frequency formats. Natural frequencies were defined as resulting from the natural sampling of events, whereby frequencies of events are recorded sequentially as those events are observed. Gigerenzer and Hoffrage (1999) further distinguished natural frequencies from frequencies originating from systematic sampling and natural frequencies that are normalised after observation. When frequencies are systematically sampled, the observer determines the base rates before any observation is made as in, for instance, scientific experimentation. Natural frequencies that are normalised after observation are expressed as ratios of positive integers with equivalent denominators. The authors argued that only the natural frequency format arising from natural sampling could facilitate Bayesian reasoning.

A typical Set-based problem in a natural frequency format reads as follows:

10 out of every 1000 women at age forty who participate in routine screening have breast cancer. 8 of every 10 women with breast cancer will get a positive mammography. 95 out of every 990 women without breast cancer will also get a positive mammography. Here is a new representative sample of women at age forty who got a positive mammography in routine screening. How many of these women do you expect to actually have breast cancer? out of . (Gigerenzer \& Hoffrage, 1995, p. 688, Table 1).

By contrast, a typical Set-based problem in a normalised frequency format reads as follows: 
10 out of every 1000 women at age forty who participate in routine screening have breast cancer. 80 of every 100 women with breast cancer will get a positive mammography. 10 out of every 100 women without breast cancer will also get a positive mammography. Here is a new representative sample of women at age forty who got a positive mammography in routine screening. How many of these women do you expect to actually have breast cancer? out of

\section{Computational accounts of judgment outcomes}

\section{Natural frequency formats lead to Bayesian judgements}

Contrary to the two previous paradigms reviewed where authors mostly described results as demonstrating neglect of base-rate information, studies in the Set-based paradigm are characterised by a high proportions of judgements matching the value given by Bayes's theorem, and as such, judgements (and participants' reasoning) are described as being Bayesian.

Gigerenzer and Hoffrage (1995) found that problems where the probabilistic information was presented in a natural frequency format led to a greater elicitation of Bayesian algorithms than problems presented in a standard probability format. The authors assessed Bayesian reasoning on the basis of both an outcome criterion-by comparing the judgement observed with its counterpart Bayesian value-as well as a process criterion-by collecting online "write aloud" protocols of participants' reasoning and/or calculations (Gigerenzer \& Hoffrage, 1995, Study 1). Overall, 46\% of the 900 judgements elicited with a task framed in a natural frequency format (60 participants $\times$ 15 problems) satisfied the joint criteria of process and accuracy. In contrast, only $16 \%$ of judgements satisfied the same joint criteria when the information was presented in a "standard probability menu;" that is, where all the information was presented in a single probability format, as in "If a woman has breast cancer, the probability is $80 \%$ that she will get a positive mammography.” (Gigerenzer \& Hoffrage, 1995, p. 688, Table 1).

These findings were seemingly corroborated by several empirical studies conducted by Cosmides and Tooby (1996), although these authors did not use natural frequency format per se (i.e., as defined by Gigerenzer \& Hoffrage, 1995, see above) but instead presented the probabilistic information using normalised natural frequencies (i.e., natural frequencies expressed as ratios of positive integers with equivalent denominators). Yet, Cosmides and Tooby (1996) found that $72 \%$ of the posterior probability judgement outcomes observed with their task matched their Bayesian counterpart. In contrast to Gigerenzer and Hoffrage (1995), Cosmides and Tooby (1996), however, only based their classification of judgements on an outcome criterion. 
It was later demonstrated that Cosmides and Tooby's (1996) results were most likely inflated by the misclassification of judgements as Bayesian on the basis of their matching of the criterion value (Evans, Handley, Perham, Over, \& Thompson, 2000; Girotto \& Gonzalez, 2001; Macchi \& Mosconi, 1998). Girotto and Gonzalez (2001), for instance, demonstrated that the proportion of judgements matching the Bayesian outcome in the task used by Cosmides and Tooby (1996) dropped from 69\% to 6\% when the numerical values presented in the problem prevented judgements resulting from an accurate evaluation of $p(H \mid D)$ being confounded with judgements resulting from other evaluations. In fact, a number of studies revealed that Set-based problems based on normalised absolute frequencies usually lead to performances comparable to those observed in Textbook problems, where the proportion of judgements matching the Bayesian outcome is usually around 10\% of the judgements observed (Evans et al., 2000; Girotto \& Gonzalez, 2001; Macchi, 2000; Macchi \& Mosconi, 1998; Mellers \& McGraw, 1999).

\section{The role of natural frequencies challenged}

Although it is now acknowledged that natural frequency formats based on natural sampling are sufficient to elicit high proportions of posterior probability judgements that coincide with the Bayesian outcome, there is evidence to show that natural frequencies are by no means necessary to the elicitation of Bayesian judgement outputs. As with the two previous paradigms, authors have started to distinguish different external factors that may contribute to the observed high proportions of Bayesian judgements in Setbased tasks.

Mellers and McGraw (1999, see also Sloman, Over, \& Slovak, 2003) argued that the characteristic of natural frequency problems was the extent to which they facilitated the visualisation of 'nested sets' rather than the (frequency) information format in which they were presented. Similarly, Evans et al. (2000) proposed that Set-based tasks facilitated the elicitation of Bayesian judgements because they "cue a simple mental model of set inclusion" (p. 200). Yet sensu stricto, both natural frequency format and normalised natural frequency formats entail nested sets. The base-rate statement, for example, which is common to both types of formats, always characterises a reference set $\mathscr{S}$ and a subset $H$. For example, in the mammography problem, the base-rate statement,

10 out of every 1000 women at age forty who participate in routine screening have breast cancer' 
which features both in a natural frequency and a normalised natural frequency task (see p. 62) defines the subset $H=\{x \mid x$ has breast cancer $\}$ nested in the reference set $\mathscr{S}=$ $\{x \mid x$ is a woman at age forty who participates in routine screening $\}$. Yet, only natural frequency formats reliably elicit Bayesian judgements, as discussed above.

Girotto and Gonzalez (2001) argued that one of the factors facilitating the elicitation of Bayesian judgements in natural frequency tasks was the "partitioned" structure of the information in natural frequency problems, where the reference set $\mathscr{S}$ is clearly subdivided into two complementary subsets, $H$ and $\sim H$, each containing another subset $D$. For example, the mammography problem presented above involves a reference set $\mathscr{S}$ defined by $\mathscr{S}=\{x \mid x$ is a woman at age forty who participates in routine screening $\}$ divided into two subsets, $H=\{x \mid x$ has breast cancer $\}$ and $\sim H=\{x \mid x$ does not have breast cancer $\}$, each containing another subset $D=\{x \mid x$ got a positive mammography\}. This description indeed best describes the critical difference between the structure of tasks presented in natural frequency formats and those presented in normalised natural frequency formats.

To show that natural frequency formats were not necessary to elicit Bayesian judgements, Girotto and Gonzalez (2001) used a partitioned chance format, which defines sets of chances (e.g., "A person who was tested had 4 chances out of 100 of having the infection," Girotto \& Gonzalez, 2001, p. 253), rather than sets of observed instances, (e.g., "4 of the 100 people tested were infected," ibid., p. 249) as in standard natural frequency problems. The authors observed similar rates of Bayesian answers with tasks presented in a natural frequency format and those presented in partitioned chance problems, thus unambiguously demonstrating that the type of elements forming the basis for the judgement (i.e., observable natural frequencies vs. abstract chances) was less crucial that the structure of the information presented for eliciting Bayesian judgements.

If the crucial difference between tasks in natural frequency format and tasks in normalised natural frequency format is structural and does not depend on the type of frequency they involve, the distinction between natural frequency vs. normalised natural frequency originally proposed by Gigerenzer and Hoffrage (1995) becomes uninformative. Accordingly, Girotto and Gonzalez (2001) distinguished between a partitioned structure, which divides a reference set into exhaustive subsets and a multiple set structure, which refers to distinct sets. 
Girotto and Gonzalez (2001) limited the role of partitioned structures in eliciting Bayesian probabilities to problems where the numerical information was presented in a ratio of integers (e.g., ' 4 out of 10 have the disease') whether the integers referred to abstract chances or frequencies of occurrence, and assimilated tasks presenting the information in a percentage format (e.g., '40\% have the disease') with multiple set tasks. Moreover, in addition to the role of such partitioned structure, they also provided evidence that the elicitation of Bayesian outputs was facilitated by the form of the question asked (i.e., by asking to produce a ratio of two absolute frequencies, as in the question asked in the mammography problem, 'How many of these women do you expect to actually have breast cancer? out of ___').

Although most of the tasks that have been reported to elicit high proportions of Bayesian judgements are consistent with these requirements, there is one exception. Namely, the results reported by Macchi (2000) suggest that Bayesian judgements may be obtained in a task where the probabilistic information is presented in a percentage form and the question, in a single-step form. Macchi (2000) indeed reported that the following task elicited Bayesian judgements from 33\% (10/30) of the participants.

360 out of every 1000 students who sit for their High School Diploma fail. $75 \%$ of the students who are not awarded a Diploma fail the written Italian paper. However, 20\% of the students who are awarded a Diploma also fail the written Italian paper.

What percentage of those who fail the written Italian paper are not awarded a Diploma? (Macchi, 2000, p. 224)

Hoffrage, Gigerenzer, Krauss, and Martignon (2002) argued that this effect was 'simply' due to the presentation of the base-rate information in absolute formats, which provides a cue to a 'natural sampling' representation. Yet, Macchi (2000) reported that the same task presenting the base-rate information in a percentage form (i.e., ' $36 \%$ of the students who sit for their High School Diploma fail') $)^{4}$ elicited Bayesian judgements from $37 \%$ (11/30) of the participants. Macchi (2000) attributed such success to the 'partitive' nature of her tasks, but as already mentioned, (see p. 60) the explicit characteristics of partitiveness have yet to be unambiguously defined. However, the issues with the partitive explanation put aside, Macchi's (2000) empirical results suggest that Bayesian judgements may be elicited with relative frequency formats and single-step questions, a

${ }^{4}$ Macchi (2000) did not explicitly report this example. It was assumed here that this formulation was used rather than '36\% out of every 1000 students who sit for their High School Diploma fail,' which added reference to the size of the set. This assumption was based on the fact that the author explicitly made the distinction between these two formulations with respect to the likelihood information where she distinguished the 'partitive' formulation " $75 \%$ of the students..." from the 'superpartitive' formulation "75\% out of 360 students who fail" (Macchi, 2000, p. 223). 
fact that remains at odds with existing accounts of the factors mediating the elicitation of Bayesian judgements.

Yet Macchi's tasks share a common characteristic with tasks framed in a natural frequency format or a partitioned chance format. Namely, the tasks used by Macchi (2000) also define a reference set $\mathscr{S}$, subdivided into two complementary subsets, each containing another subset (i.e., $\mathscr{S}=\{x \mid x$ is a student who sits for his High School Diploma $\}$, subdivided into $H=\{x \mid x$ failed/is not awarded the diploma $\}$ and $\sim H=\{x$ $\mid x$ is awarded a diploma $\}$, each containing the subset $D=\{x \mid x$ failed the Italian paper\}). This suggests that the importance of the 'partitioned' structure, as clearly defined by Girotto and Gonzalez (2001) may also mediate Bayesian performance in tasks where both the probabilistic information and the experimental question are presented in a percentage (i.e., relative frequency) format. In the remainder of the thesis the term "fully inclusive structure" will be used to refer to both unit partitioning as defined by Girotto and Gonzalez (2001) and percentage partitioning, as illustrated by Macchi's (2000) task.

Both multiple set and fully inclusive structures are represented graphically in Figure III-3.

Multiple set structure

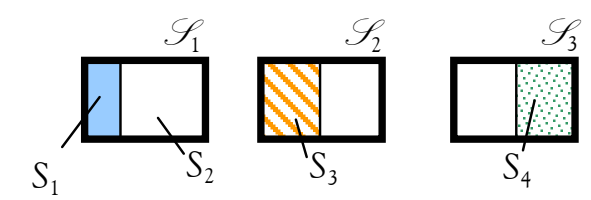

$\mathscr{S}_{1}=\{x \mid x$ is a woman undertaking breast screening $\}$

$S_{1}=\{x \mid x$ has breast cancer $\}$

$S_{2}=\{x \mid x$ is healthy $\}$

$\mathscr{S}_{2}=\{x \mid x$ has breast cancer $\}$

$S_{3}=\{x \mid x$ has got a positive mammogram $\}$

$\mathscr{S}_{3}=\{x \mid x$ is healthy $\}$

$S_{4}=\{x \mid x$ has got a positive mammogram $\}$
Fully inclusive structure

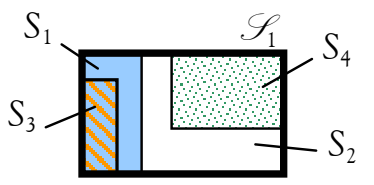

$\mathscr{S}_{1}=\{x \mid x$ is a woman undertaking breast screening $\}$

$S_{1}=\{x \mid x$ has breast cancer $\}$

$S_{2}=\{x \mid x$ is healthy $\}$

$S_{3}=\{x \mid x$ has got a positive mammogram $\}$

$S_{4}=\{x \mid x$ has got a positive mammogram $\}$

Figure III-3 - Venn diagrams of fully inclusive vs. multiple set information structure in the mammography problem.

Note. Venn diagrams represent the logical relationship of between sets (inclusion, exclusion, intersection) as well as information relative to the area of the sets represented (i.e., mutual exclusivity and collective exhaustiveness).

\section{Can we tell that individuals are Bayesian reasoners?}

The issues discussed so far are characteristic of an outcome-focused approach to the study of posterior probability judgements whose goal is the identification of the factors that mediate the elicitation of normative outputs. By contrast, the process-focused 
approach followed in this thesis sets a different research agenda, whose primary aim is to provide a systematic computational description of the judgement methods underlying observed outputs, in order to foster the subsequent development of a sound algorithmic account of the cognitive processes that underpin the application of such methods. From this perspective, the issue of importance becomes what underlies the responses observed rather than what may or may not elicit a certain response. This approach leads to the identification of yet another issue that has not received much attention. In most research work within the Set-based paradigm, researchers seemed to endorse, either explicitly or implicitly, the conclusion that Bayesian outputs necessarily point towards underlying Bayesian reasoning processes (e.g., see Gigerenzer \& Hoffrage, 1995). In other words, from the observation that (fully inclusive) Set-based tasks elicited high proportions of judgement outputs coinciding with their counterpart Bayesian outcome, authors concluded that those tasks facilitated Bayesian reasoning.

From a normative perspective, these results introduced a new turn in the discussion of the rationality of human posterior probability judgement as judges were redeemed as Bayesian after all (e.g., see Cosmides \& Tooby, 1996). If one's goal is to demonstrate whether individuals can demonstrate normative behaviour, then the Set-based paradigm appears to provide the definite answer. The normative approach has its limitations, however, as Cosmides and Tooby's (1996) views on psychologists' objectives paradoxically illustrate:

When psychologists (including ourselves) argue about whether people do, or do not, engage in Bayesian reasoning, they are discussing the extent to which our inductive reasoning mechanisms map inputs onto the same outputs that Bayes' rule would, regardless of the actual cognitive procedures employed to accomplish this. [italics added] (p. 8)

The authors justify their claim on the basis that past experiments seldom if ever attempted to determine what such cognitive processes were. Thus, when researchers' agenda is to determine whether human posterior probability judgements are in line with normative prescriptions, the scope of the psychological study of such judgements is confined to a behaviourist approach and the psychological significance of the specification of underlying cognitive mechanisms is simply ruled out. Yet, in contrast with Cosmides and Tooby's (1996) views (but in line with a few other psychologists' explicit views, e.g., see Einhorn \& Hogarth, 1981; Hamm, 1998; Hertwig \& Todd, 2000; Krueger, 1998; Slovic \& Lichtenstein, 1971), the process-focused approach developed here precisely posits the uncovering of those cognitive processes underlying observed responses as the focal point of research on probabilistic inferences. By taking this 
cognitivist approach, the limitations of the outcome-focused perspective- which, as we saw, allows for the bypass of the study of those processes-are manifest while the potentials of a process-focused perspective are best revealed. Indeed, if one's goal is to understand the mechanisms that underlie observed probability judgements, whether or not an output is normative is beside the point. In contrast, from a process-focused perspective, the crucial issue raised by the results observed within the Set-based judgement paradigm becomes the identification of the reasoning processes underlying the numerical outputs observed in Set-based tasks.

Can one infer from the observation of Bayesian outputs-i.e., numerical outputs matching values calculated with Bayes's theorem-that individuals' reasoning processes were Bayesian? So far, the evidence advanced in the present thesis suggests that there are often several possible accounts for a given numerical output. Similarly, one should be cautious when taking Bayesian outputs as evidence for underlying Bayesian reasoning. As Girotto and Gonzalez (2001) noted, "wrong conclusions may appear as correct solutions" (p. 265); the observation of a numerical output that matches that of a specific method indeed does not necessarily imply that the reasoning processes underlying the judgement observed corresponds to the method itself (see also Macchi, 1995).

Gigerenzer and Hoffrage (1995) recognised the limitations of studies whose findings exclusively rely on outcome analysis and proposed to couple the measure of probability or frequency estimates with on-line protocols to allow for process analysis in addition to outcome analysis. The authors thus classified inferential processes observed as Bayesian algorithms ${ }^{5}$ if outputs exactly matched their corresponding Bayesian value and protocols provided evidence that a Bayesian computation had been used. Yet can the use of a Bayesian algorithm be taken as evidence of Bayesian reasoning? Compare the two following definitions (from the Chambers Encyclopedic English Dictionary, 1994):

(1) Algorithm. A process or set of rules used for solving problems, calculations, etc.

(2) Reasoning. The forming of judgements or opinions using reason or careful argument.

Although both concepts refer to processes, the algorithm is computational and results in a single numerical output, whereas reasoning is more 'natural' and can result in a conclusion with a relative degree of confidence. In other words, one could be induced to apply an algorithm without necessarily demonstrating reasoning. A stronger version of

${ }^{5}$ The term Bayesian algorithm should not be confounded with Marr's (1982) algorithmic level of analysis. The issue as to whether individuals' judgements reflect the application of a Bayesian algorithm rather than result from the use of Bayesian reasoning still pertains to the computational level of analysis, which is aimed at identifying what individuals do when making their judgements. 
this argument has been reported within the literature. Concerning Cosmides and Tooby's (1996) claim that correct Bayesian reasoning can be elicited by the use of a frequency format, Howson and Urbach (1993) commented:

To claim that people are statistical reasoners because they can get the right answer to the disease problem when it is posed in terms of numbers ... is fallacious, and the data tells us little except ... about the subjects' ability to do arithmetic. (p. 422)

Although the argument is somewhat forceful, a simple illustration can reveal its strength. Consider Gigerenzer and Hoffrage's (1995) mammography problem in its natural frequency format (see p. 62). According to the authors' outcome and process criteria, if participants in this condition provided both the correct numerical answer and reportedly arrived at this estimate by computing the appropriate computations, their answer would be classified as Bayesian. Now, consider the following adaptation of the previous problem:

8 women with breast cancer will also get a positive mammography. 95 women without breast cancer will also get a positive mammography. Out of the number of women with a positive mammography, how many will actually have breast cancer? out of

Most individuals should be able to provide the "correct" answer to this problem. Moreover, accompanying on-line protocols should be similar to those observed in Gigerenzer and Hoffrage's (1995) original task; namely, these protocols would indicate that participants evaluated the number of women with breast cancer out of the total number of women with a positive mammography. Yet, in the absence of information on the relative size of each sub-population (i.e., the total number of women with and without breast cancer), the numerical information can no longer be assumed to approximate the "true" frequencies within the general population. As such, the resulting judgements can no longer be assimilated with probability judgements: they are no more than the arithmetical computation of a relative frequency. Consequently, it is only natural that algorithms observed in the latter version of the task would not be labelled "Bayesian algorithms", let alone "Bayesian reasoning." Yet, that the underlying cognitive processes are similar in both tasks certainly remains conceivable.

There is no question that fully inclusive information structures help to elicit cognitive algorithms in accordance with Bayes's theorem. Similarly, it is apparent that those algorithms can be externalised by participants in their on-line protocols. Yet, as illustrated by the previous example, the extent to which these algorithms reflect Bayesian reasoning is quite weak. So what evidence would be suitable to demonstrate 
Bayesian reasoning in posterior probability judgement tasks? ${ }^{6}$ In other words, when can we assume that the computation of the Bayesian output observed is the result of a purposeful and conscious inference rather than the result of the mechanical application of an algorithm?

Gigerenzer and Hoffrage (1995) themselves gave quite an insightful—yet fictitiousexample of human Bayesian reasoning,

Imagine an old, experienced physician in an illiterate society. She has no books or statistical surveys and therefore must rely solely on her experience. Her people have been afflicted by a previously unknown and severe disease. Fortunately, the physician has discovered a symptom that signals the disease, although not with certainty. In her lifetime, she has seen 1,000 people, 10 of whom had the disease. Of those 10, 8 showed the symptom; of the 990 not afflicted, $95 \mathrm{did}$. Now a new patient appears. He has the symptom. What is the probability that he has the disease? (pp. 686-687)

In this example, the authors describe the physician's natural sampling of occurrences within a given population. In this case, like in natural frequency Set-based tasks, the information is available in a natural frequency format. Consequently, the posterior probability can be computed by directly evaluating the ratio of the number of cases presenting both the symptom and the disease to the total number of cases presenting the symptom, without the need for integrating the base-rate information (10 out of 1000 people have the disease). In contrast with natural frequency tasks, however, the physician is not prompted to compute this ratio by a "two-step" question, such as the question included in natural frequency tasks; that is, she is not asked, "How many of the people with the symptom, do you expect to actually have the disease? out of _.." Instead, she is prompted to evaluate the single-event probability that the patient who presents the symptom actually has the disease.

Accordingly, the physician depicted in this example needs to infer that, in order to evaluate the single-event probability that a patient who presents the symptom actually has the disease, she needs to recall and evaluate the ratio of the number of her patients who presented the disease out of the number of patients who had the symptom. In other words, she needs to hold and apply the "relative frequency rule" (Teigen, 1994; see

${ }^{6}$ This restriction to posterior probability judgement tasks is not trivial. Its purpose is to distinguish between Bayesian reasoning or belief revision in scientific hypothesis testing (e.g., see Howson \& Urbach, 1993) and what can be characterised as Bayesian reasoning in "one-shot" posterior probability judgement tasks. Bayesian reasoning in the former domain encompasses the philosophical and mathematical issues associated with the revision of one's prior belief, defined by the prior probability $p(H)$, in the light of new evidence. By contrast, the characterisation of Bayesian reasoning in one-shot posterior probability judgement tasks, which are the object of study of the present thesis, consists in distinguishing the reasoning processes from the purely arithmetical processes involved in evaluating the conditional probability, $p(H \mid D)$, given the evidence available. 
chap. II, p. 30), which stipulates that the probability of an outcome occurring is equal to its past relative frequency of occurrence. Thus, in this case, Bayesian reasoning demands more than the simple application of a Bayesian algorithm entailing the calculation of the appropriate ratio of integers. Specifically, Bayesian reasoning necessitates the realisation that such an algorithm is needed in order to evaluate the value of the single-event probability required. This realisation itself firstly presupposes the Popperian ${ }^{7}$ intuition that the probability of the singular event (i.e., the intuition that the new patient has the disease given he has the symptom) is equal to the relative frequency of occurrence of the phenomenon (i.e., the relative frequency of occurrence of the disease among previous patients presenting the symptom). Secondly, Bayesian reasoning also demands the appropriate phenomenon to be considered. If the physician had considered the number of her patients who had the disease and estimated the number of those patients who also had the symptoms, her reasoning would have entailed the estimation of the inverse probability and as such, it would not have been an instance of Bayesian reasoning.

Yet it is a normative assumption to presume that the experienced but illiterate physician will rely on a Popperian concept of probability, whereby the probability of an event is equated to its past relative frequency of occurrence. There are as many other types of Bayesian reasoning as there are possible interpretations of probabilities. One could also evaluate the chances that $H$ is true given that $D$ is true, where such chances correspond to degrees of belief and may or may not be solely based on frequency considerations. Thus, the results obtained in partitioned Set-based tasks (either using a natural frequency format or using a partitioned chance format) undeniably elicit Bayesian algorithms; that is, algorithms involving the appropriate evaluation of the number of $H \& D$ cases out of the number of $D$ cases, when they are prompted to evaluate these very numbers. Yet, the question as to whether people understand that this ratio represents the posterior probability $p(H \mid D)$ remains open. More importantly, the question as to whether people can demonstrate Bayesian reasoning; that is, whether they can reason that, in a situation such as the one faced by the old physician whose explicit goal was to evaluate the posterior probability $p(H \mid D)$, they need to apply such a Bayesian algorithm, remains to be answered.

\footnotetext{
${ }^{7}$ see section on the Propensity theory of probability, chap. II, p. 25.
} 


\section{Algorithmic accounts of underlying reasoning processes}

\section{The evolutionary hypothesis}

Apart from examining whether current descriptions of posterior probability judgements within the Set-based paradigm adequately reflect individuals' behaviours in those tasks, the process-focused approach followed here also entails the examination of the algorithmic accounts explaining the cognitive underpinnings of the judgements observed. There is no question that fully inclusive Set-based problems entail a large proportion of judgements based on the utilisation of a Bayesian algorithm. Available accounts for this finding, however, diverge. Gigerenzer and Hoffrage (1995) proposed that we possess mental algorithms-later defined by Gigerenzer (1997) as, "induction mechanisms that perform classification, estimation, or other forms of uncertain inferences, such as deciding what color an object is, or inferring whether a person has a disease" (p. 110). In particular, Gigerenzer and Hoffrage (1995) argued that, with regards to statistical inference, we have evolved cognitive algorithms that are specifically adapted for natural frequencies; that is, absolute frequencies arising from natural sampling. This claim relies on the evolutionary assumption that organisms evolve mechanisms that are tailored to the structure of their external environment. And, our environment, the authors further argued, has presented us with frequency information long before the concepts of probability or percentage came into place, thus making the natural frequency format, the natural format for encoding and representing numerical information (Gigerenzer \& Hoffrage, 1995, see also, e.g., Gigerenzer, 1996, 1997; Cosmides \& Tooby, 1996).

Yet authors who demonstrated that the facilitating effect was not due to the statistical format of the information per se have challenged the evolutionary explanation. These authors proposed instead that the mental representation and interpretation of the task-induced by the specific structure of the information and the specific format of the question asked—was responsible for eliciting Bayesian responses (Evans et al., 2000; Girotto \& Gonzalez, 2001, 2002). Therefore, as for the two previous paradigms, these accounts suggest that to understand why a particular judgement has been elicited, one need to understand what task representation was elicited from the task at hand.

Evans et al. (2000) proposed that Set-based tasks facilitated the elicitation of Bayesian judgements because they "cue a simple mental model of set inclusion by focusing every statistic on a group of 1000 people" (p. 200). The authors, however, 
neither further stipulated what such 'mental models of set inclusion' consisted of, nor specified the modalities by which the task would elicit such representation. Moreover, their account suggests that any Set-based task framed in an absolute format would cue such an inclusion model because of the presence of a common statistic. This claim is in sharp contrast with findings that multiple Set-based tasks-whose sample sizes are normalised and, hence, refer to a common statistic_do not facilitate Bayesian reasoning (see, e.g., Girotto \& Gonzalez, 2001).

\section{The Mental Model Theory of probabilistic reasoning}

Girotto and Gonzalez (2001) proposed that the external structure of the information presented in a problem "determines the way in which information is represented" and the form of the question asked, "determines the inferences that reasoners draw based on the representation they have constructed" (p. 268). The authors based their account on the "mental model theory (MMT) of probabilistic reasoning" (Johnson-Laird et al., 1999) and in particular, argued that the theory's subset principle accounted for Bayesian performances in Set-based problems, whether frequencies referred to natural frequencies or partitioned chances.

The MMT of probabilistic reasoning models mental representations of individuals who engage in extensional probabilistic reasoning; that is, individuals who will infer the probability of an event from the different possible ways in which the event could occur. By contrast, non-extensional reasoning is defined as the inductive inference of a probability based on "some relevant heuristic, index, or evidence" (Johnson-Laird et al., 1999, p. 63). The theory proposes a first, truth principle, according to which "People represent situations by constructing sets of mental models in which each model represents what is true in a true possibility" (p. 68). Accordingly, if told that "2 out of every 5 people have a disease", individuals will form a representation including five mental models, two representing an individual with a disease, and another three models to account for the three people who do not have a disease. Such a representation can be formalised as follows:

$\begin{array}{ll}\text { people } & \text { disease } \\ \text { people } & \text { disease } \\ \text { people } & \\ \text { people } & \\ \text { people } & \end{array}$

Each model represents one instance or one individual. Johnson-Laird (1983; see also Johnson-Laird \& Byrne, 1993) indeed contended that mental models of quantified 
predicates such as 'All artists are beekeepers' "represent finite sets of entities by finite sets of mental tokens rather than by circles inscribed in Euclidean space" (p. 329).

The second principle, or equiprobability principle states, "Each model represents an equiprobable alternative unless individuals have knowledge or beliefs to the contrary, in which case they will assign different probabilities to different models" (Johnson-Laird et al., 1999, p. 68). According to this principle, in the absence of any other information, people would assume that models [1], [2], [3], [4], and [5] are equally likely to occur.

Granted equiprobability, the evaluation of the probability of the event is accounted for by the proportionality principle, according to which the probability of the event "disease" thus depends on the proportion of models in which it occurs. When premises refer to numerical probabilities, the numerical principle states that models can be "tagged with their appropriate numerical values, and an unknown probability can be calculated by subtracting the sum of the $(\mathrm{n}-1)$ known probabilities from the overall probability of the $\mathrm{n}$ possibilities in the partition" (p. 69). Models can thus be "tagged" with single probabilities, but also frequencies, percentages, etc. So the statement, "there is a $40 \%$ probability that a person has a disease" can be represented by the following models:

$$
\text { person } \quad \text { disease } \quad 40 \%
$$

where an implicit model (denoted by the ellipsis) represents individuals who do not have the disease.

Thus, according to those principles, a standard fully inclusive Set-based problem (e.g., see p. 62) will entail the following representation:

$\begin{array}{ccc} & & \text { Frequency } \\ \text { breast_cancer } & \text { positive_test } & 8 \\ \text { breast_cancer } & \neg \text { positive_test } & 2 \\ \neg \text { breast_cancer } & \text { positive_test } & 95 \\ & \ldots & 895\end{array}$

where the implicit model represents the individuals who neither had breast cancer nor had a positive mammogram. To account for posterior probability judgements, MMT proposes that individuals will follow the subset principle,

Granted equiprobability, a conditional probability, $p(A \mid B)$, depends on the subset of $B$ that is $A$, and the proportionality of $A$ to $B$ yields the numerical value. Otherwise, if the models are tagged with their absolute frequencies (or chances), then the conditional probability equals the frequency (chance) of the model of $A$ and $B$. In computing the ratio of the subset relation, reasoners can err in assessing either the numerator or, more likely, the denominator. (Johnson-Laird et al., 1999, p. 80) 
Thus, according to the MMT principles, the representation above would entail the elicitation of a Bayesian judgement of the posterior probability $p$ (breast_cancer|positive_test), as individuals, following the subset principle, will evaluate the proportionality of models featuring "breast_cancer" to those featuring "positive_test", by reviewing the numerical tags. Namely, in the example above, individuals will assess that there are 8 models featuring "breast_cancer" within a total of $103(8+95)$ models featuring "positive_test." In contrast, the same problem presented in a single-probability format would require the following fully explicit mental model:

\begin{tabular}{cccc} 
& Probability & \multicolumn{2}{c}{ Conditional probability } \\
breast_cancer & $1 \%$ & positive_test & $80 \%$ \\
& & $\neg$ positive_test & $20 \%$ \\
$\neg$ breast_cancer & $99 \%$ & positive_test & $9.6 \%$ \\
& & positive_test & $90.4 \%$
\end{tabular}

Johnson-Laird et al. (1999) concluded that even if such representations are elicited, they do not clearly point to the posterior probability. Thus, according to the MMT account of probabilistic reasoning, "The numerical version of the subset principle applies only to absolute frequencies or chances, not to conditional probabilities, and so the only route to the answer [in single-probability tasks] appears to be to use Bayes's theorem" (p. 80).

Mental Model Theory is a sound process-focused account of how we may reason about possibilities (e.g., the possibility that there is a king in a hand of cards, the possibility of a six facing up when a dice is thrown). Nevertheless, it may lack the grounds for adequately accounting for the ways in which we reason about probabilities as relative frequencies. This distinction is in line with Teigen's (1994) taxonomy of rules that may be used as a basis for probability judgements (see Chapter II, p. 30). Teigen indeed distinguished between probability judgements based on the use of a 'proportion' rule and that of a ' $1 / n$ ' rule and probability judgements based on a 'relative frequency' rule.

MMT stipulates that individuals are unlikely to represent fully explicit mental models of standard posterior probability tasks, because of the strain such models entail on memory workload. Nevertheless, assuming that individuals represent a model for every proposition in the task, one may predict, on the basis of the MMT principles, that this representation would be as follows, 


\section{breast_cancer \\ breast_cancer \\ breast_cancer \\ $\neg$ breast_cancer}

\begin{tabular}{rr} 
& \multicolumn{2}{r}{ Conditional p } \\
& $1 \%$ \\
positive_test & $80 \%$ \\
$\neg$ positive_test & $20 \%$ \\
positive_test & $9.6 \%$
\end{tabular}

The literature has shown that this type of problem leads people to evaluate the posterior probability $p$ (breast_cancer|positive_test) to be equal to this inverse probability $p$ (positive_test|breast_cancer), in this case, $80 \%$. If taken sensu stricto, the proportionality principle and the subset principle would indicate that people evaluate the probabilities associated with the proportions of models that feature "breast_cancer" relative to the probabilities associated with models featuring "positive_test". Yet the empirical evidence clearly suggests that people do not apply the subset principle in this case. The authors were careful to integrate this empirical finding in their account by limiting the application of the subset principle to cases where the numerical information is presented in absolute terms (i.e., either frequencies or chances). Nevertheless, this limitation does not provide an explanation as to why people do not apply the subset principle when information is presented in a probability format.

Moreover, the authors proposed that Bayesian judgements will only be elicited from a large proportion of individuals when the information is both presented in an absolute numerical format (to warrant the application of the subset principle) and partitioned in such a way that the application of the subset principle will indeed result in the evaluation of the correct probability. This latter condition thus accounts for the fact that multiple set tasks do not elicit Bayesian judgements, even if they are framed in an absolute numerical format, whether based on frequencies or chances. Yet there is empirical evidence to suggest that relative frequency formats can also lead to Bayesian judgements (see Macchi, 2000), a finding that is not accounted for by the MMT, even if the domain of application of the subset principle were extended to relative frequencies.

In sum, the MMT account of probabilistic reasoning provides a powerful explanation to further our understanding of the processes underlying reasoning about possibilities. Yet some drawbacks were identified in relation to the theory's account of the processes underlying posterior probability judgements. In particular, the theory in its current state posits that judgements will be determined by the subset principle only when they are based on statements in an absolute numerical format. Although this limitation is consistent with existing empirical findings, the principles of the theory do not explain 
why this limitation is necessary. Additionally, the MMT, in its current state, precludes the possibility of observing Bayesian judgements in tasks framed in a relative frequency formats. Yet there is evidence to suggest that even relative frequency formats may elicit Bayesian judgements.

\section{Conclusions}

The Set-based paradigm is the latest paradigm within which psychologists have studied posterior probability judgements. Empirical results suggest that a specific type of Set-based tasks-the fully inclusive Set-based tasks-elicit high rates of Bayesian judgements. Thus, fully inclusive information structure (that is, either fully inclusive frequencies or fully inclusive chances) provides a promising answer to the outcomefocused approach to the study of posterior probability judgements. Within this approach, researchers have indeed aimed at identifying the factors that undermine normative judgements. Although fully inclusive Set-based tasks 'only' elicit Bayesian judgements from an average of $40 \%$ participants, it is a substantial increase compared to the usual percentage of Bayesian judgements observed in studies within the Textbook-problem paradigm, for instance.

From the process-focused approach followed here, however, the primary issue of importance is no longer the rate of judgements matching the Bayesian criterion. Instead, the issue at stake is whether the description of judgements as 'Bayesian' is an adequate description of the cognitive processes that underlie those judgements. Hence, it appears that whereas some authors have equated Bayesian reasoning with the application of a Bayesian algorithm, others dispute this assimilation. This observation, in turn, calls for both a theoretical outline of what Bayesian reasoning entails and an empirical investigation of the extent to which individuals indeed can exhibit such types of reasoning in posterior probability judgement tasks.

Following the thorough description of what judgement methods lead to the numerical outputs observed, the process-focused approach aims at explaining how such methods are elicited by the task. The two main types of account of posterior probability judgements within the Set-based paradigm are accounts based on the evolutionary hypothesis on the one hand, and accounts invoking mental representations of the task on the other hand. The former hypothesis states that fully inclusive frequency formats lead to Bayesian judgements because we have evolved statistical mechanisms tailored to frequencies of instances rather than probabilities. This hypothesis has been challenged 
by empirical results demonstrating that Bayesian judgements can also be elicited by formats other than the natural frequency one (e.g., by the partitioned absolute chance format). The mental representation hypothesis-according to which it is the representation of the task and the interpretation of the question-was proposed as an alternative to the evolutionary account. From a process-focused approach to posterior probability judgements, this latter theoretical framework thus appears auspicious. Existing accounts, however, suffer from some limitations. Some (e.g., Evans et al., 2000) allude to the concept of representation without further formalising what such representations consist of, or specifically explaining how such representations may mediate the application of Bayesian algorithms. For the first time within the literature, a formalised detailed account of the role of representations in probabilistic reasoning was provided (Johnson-Laird et al., 1999). Yet it was contended that, in its current state, this account still suffers from shortcomings, if only concerning the explanation of the processes underlying posterior probability judgements.

\section{GENERAL CONCLUSIONS}

Following a brief introduction to the historical context of the study of posterior probability judgements-distinguishing between earlier conservatism studies and the subsequent establishment of the heuristics and biases framework-the objective of this chapter was to critically review the state of knowledge concerning posterior probability judgements, from a process-focused perspective. Such a perspective entailed the systematic review of (i) the existing computational accounts of the processes underlying posterior probability judgements, as well as (ii) the existing algorithmic accounts explaining the origin of such judgemental processes. This analysis uncovered issues that have been overlooked, probably because they are somewhat irrelevant to the outcomefocused approach, which has dominated the study of posterior probability judgements so far. Yet these issues are crucial to the process-focused approach delineated in the present thesis.

\section{Synthesis of available computational accounts of the processes underlying posterior probability judgements}

This systematic review was organised around the three main paradigms within which researchers have studied posterior probability judgements for the past three decades, 
namely, the Social-judgement paradigm, the Textbook-problem paradigm and the Setbased paradigm.

Despite the dissimilarities between Social-judgement and Textbook-problem tasks, the review identified similar issues relevant to the process-focused approach. Within both paradigms, one may indeed question the extent to which the 'base-rate neglect' hypothesis appropriately describes participants' behaviour. The inverse fallacy; that is, the tendency to equate posterior probabilities with their inverse likelihood, appears to be a better candidate for describing what individuals may do when making posterior probability judgements in those tasks. Yet inverse and base-rate fallacy computational accounts have yet to be empirically distinguished within each of these paradigms. The representativeness heuristic has also been identified as an alternative account to describe the processes underlying judgements within the Social-judgement paradigm. Therefore, in this latter paradigm, it is not two but three alternative computational accounts that need to be empirically distinguished: the base-rate neglect, the inverse fallacy and the representativeness heuristic accounts. A similar issue arose within the Set-based paradigm, where the extent to which evidence of the application of a Bayesian algorithm can be taken as evidence of Bayesian reasoning was challenged. From a process-focused perspective, this contention highlights the need for a clearer theoretical definition of what Bayesian reasoning entails as well as the need for empirical evidence distinguishing between the application of a Bayesian algorithm and reliance on Bayesian reasoning.

The review also revealed that the validity of our knowledge of what posterior probability judgements may entail was also possibly undermined by the statistics (e.g., mean outputs) upon which conclusions have been drawn, especially in the Socialjudgement paradigm. As a result, the question of whether the base-rate neglect is representative of individual judgemental strategies remains open. A related issue was outlined within the Textbook paradigm where the extent of base-rate usage has been evaluated based on statistical measures such as linear regression weight models or inferred when judgements' outputs lay between inverse and base-rate values. Studies within this latter paradigm, however, generally provided idiographic information, thereby allowing for more insight into what individuals may have done in making their judgements. Yet while the spread of the judgements' outputs observed points towards the existence of individual differences, computational accounts available have usually focused on the modal and the normative output(s) observed. 
This is an instance where the relevance of the issue of interest will diverge as a function of the approach favoured by the researcher. Within an outcome-focused approach, authors are concerned with the accuracy of outputs observed, and therefore need not to distinguish between different inaccurate judgements. From a processfocused approach, however, since one would ultimately aim at providing an algorithmic account of judgements which would describe how individuals make their judgements, it is necessary to first develop a valid and reliable computational account describing what individuals do, whether or not their judgements are in line with normative prescriptions.

Finally, another factor that was identified as having possibly hindered the descriptive adequacy of results available within both the Textbook and the Social-judgement paradigm was the assumption of additivity. This assumption has led researchers to recode estimates of $p(\sim H \mid D)$ as estimates of $p(\mathrm{H} \mid \mathrm{D})$ by subtracting the former from 1. Yet this assumption is at odds with the empirical evidence suggesting that individuals do not follow the additivity principle. Within the context of posterior probability judgements, if the inverse fallacy can account for the judgements observed-that is, if $p(H \mid D)$ and $p(\sim H \mid D)$ judgements are judged to be equal to the value of $p(D \mid H)$ and $p(D \mid \sim H)$, respectively - then deviations from additivity of the sum of $p(H \mid D)$ and $p(\sim H \mid D)$ judgements should be predictable from the sum of $p(D \mid H)$ and $p(D \mid \sim H)$ judgements (in the Social-judgement paradigm) or values provided in the text (in the Textbook paradigm).

\section{Synthesis of available algorithmic accounts of the determinants of posterior probability judgements}

Among the accounts reviewed within each of the paradigms, two main determinants for the judgemental processes delineated were identified, namely, the role of the interpretation of the task and the role of individuals' mental representation of the task. Within both the Social-judgement and the Textbook-problem paradigms, authors attempted to provide an explanation for the neglect of the base-rate information. The validity of such accounts therefore depends on the assumption that the base-rate neglect' hypothesis actually describes behaviours. Such an assumption is not, as the previous section revealed, without shortcomings.

Within the Social-judgement paradigm, the representativeness heuristic was relegated to a computational account of judgement behaviours. An alternative cognitive account emerged from the literature. Namely, it was proposed that the representation of 
the task and its interpretation invoked particular cognitive strategies, themselves determining the type of judgement behaviour observed (i.e., e.g., the integration of baserate information in the final judgement or a judgement based on the representativeness heuristic). Yet the empirical tests of such accounts were based on average judgements, making it impossible to determine whether individuals actually relied on either of these strategies or any other. In addition, although these accounts emphasized the role of the representation of the task, no formal description of what such a representation may comprise was proposed within this paradigm of research.

The role of individuals' interpretations was also at the core of algorithmic accounts occurring within the Textbook paradigm. These interpretational accounts suggested that it was the interpretations evoked by the task that determined the way attention was distributed to the different elements of the problem in general, and the base-rate information in particular. Yet again, those accounts did not clearly specify the exact modalities by which factors such as causality, relevance or the verbal structure of the text may determine the final judgement.

Finally, the role of mental representation was emphasized in algorithmic accounts aiming at explaining the origin of the 'Bayesian' judgements observed in 'fully inclusive' Set-based tasks. The most systematic account is the Mental Model Theory account of probability judgement, which extended to posterior probabilities (Johnson-Laird et al., 1999). Yet it was suggested that the MMT account still suffers from shortcomings, if only in relation to the account of the origin of the processes underlying posterior probability judgements.

The empirical issues identified by the review of computational accounts of posterior probability judgements within the Social-judgement, Textbook-problem and Set-based paradigms are addressed in the chapters IV, V, and VI of the present thesis, respectively. The theoretical issues identified in relation to the development of an algorithmic account of posterior probability judgements are addressed in Chapter VII when a new theoretical account, called Sample Space Representation theory, is introduced.

\section{sk}


7 he objective of this thesis is to develop our understanding of posterior probability judgements from a process-focused perspective. Most studies investigating posterior probability judgements have followed an outcome-focused perspective, as their aim was to uncover the conditions that mediate Bayesian performance. The process-focused perspective is an alternative approach, which aims to (i) provide a computational account of posterior probability judgements; that is, a systematic description of the judgement methods applied, whether they lead to the production of a normative outcome or not, and (ii) to develop an algorithmic account explaining how such methods are elicited by the task. The Social-judgement paradigm involves the elicitation of posterior probability judgements based on a numerical baserate statement and a verbal description of the data. In chapter III, the review of the literature on posterior probability judgements within this paradigm revealed the existence of three different accounts for describing the processes underlying judgement outcomes. Social-judgement experiments indeed have usually revealed that outcomes of judgements such as the probability that this person, who is trustworthy, is an engineer reflected the 'neglect of base-rate information' (Kahneman \& Tversky, 1973). That is, people neglect the information about the proportion of engineers in the population under consideration. Such a descriptive account, however, only emphasises what does not affect judgements, thereby allowing for a wealth of possibilities to account for what does underlie judgements.

The representativeness heuristic, defined as the tendency to evaluate the posterior probability $p(H \mid D)$ on the extent to which an instance described by $D$ is representative of the members of category $H$, was originally proposed as an explanatory account. Yet, this heuristic is now recognised as an alternative descriptive account of judgement outcomes within the Social-judgement paradigm (e.g., see Tversky \& Kahneman 1982c). Still, this descriptive account has been criticised for its lack of a clear-cut definition of its object, namely the concept of 'representativeness' (e.g., Gigerenzer \& 
Hoffrage, 1995; Wallsten, 1983) and its post-hoc explanatory nature (e.g., Gigerenzer, 1996; Groner, Groner \& Bischof, 1983; Wallsten, 1980). Finally, yet another account has been proposed to account for outcomes observed in Social-judgement tasks. Namely, it has been suggested that judgements of representativeness are nothing but evaluations of $p(D \mid H)$, the likelihood of the data, when the hypothesis is known to be true (Gigerenzer \& Murray, 1987; Pollard \& Evans, 1983). For example, this latter account suggests that when asked to evaluate the probability that ' $x$ is a lawyer given that $x$ is trustworthy', individuals in fact evaluate the probability that ' $x$ is trustworthy given that $x$ is a lawyer.' This latter account can be assimilated to the 'inverse fallacy', which has originally been defined within the Textbook-problem paradigm (Koehler, 1996).

To date, however, there is no empirical evidence to distinguish between base-rate neglect, representativeness and inverse fallacy as potential descriptors of the processes underlying judgement outcomes observed within the Social-judgement paradigm. Yet, such a distinction is an essential underpinning for the development of an algorithmic account of posterior probability judgements - the ultimate aim of the process-focused approach followed herein. Another computational issue is related to the additivity of posterior probability judgements of binary complementary hypotheses. There is evidence to show that people generally do not respect the additivity principle when asked to judge the probability of multiple complementary hypotheses (Edwards, 1954; Teigen, 1974). With regards to binary complementary hypotheses, conflicting evidence has been reported (see Ayton, 1997; Macchi et al., 1999; Rottenstreich \& Tversky, 1997; Tversky \& Koehler, 1994). In any case, it appears that posterior probability judgements of binary complementary hypotheses in Social-judgement tasks are not additive (Baratgin \& Noveck, 2000). Yet, there is little evidence as to what exactly determines such departures from additivity. This demonstrates the importance of the clarification of the processes which underlie posterior probability judgements in those tasks. For example, the inverse fallacy consists in evaluating $p(D \mid H)$ when asked to judge $p(H \mid D)$, or, evaluating $p(D \mid \sim H)$ when asked to judge $p(\sim H \mid D)$. If people commit the inverse fallacy, then the extent to which the sum of their judgements of $p(H \mid D)$ and $p(\sim H \mid D)$ will be subadditive (i.e., greater than one) or superadditive (i.e., less than one, as defined in Tversky \& Koehler, 1994) should be predictable from the sum of their judgements of the likelihoods $p(D \mid H)$ and $p(D \mid \sim H)$.

The present study was therefore aimed at establishing empirically whether the inverse fallacy could describe posterior probability judgement outcomes observed within 
the Social-judgement paradigm. A second objective was to investigate the extent to which the magnitude and direction of deviations from additivity could be predicted from inverse likelihood estimates. A scenario adapted from the lawyer-engineer problem was used. It involved two mutually exclusive and collectively exhaustive categories; the 'designers' category $(H)$ and the 'accountant' category $(\sim H)$. To control for the diagnosticity of the verbal descriptions presented, stereotypical traits and hobbies associated with each of these two professional categories were first elicited from a sample of participants. On the basis of this information, five descriptions were constructed, and their diagnosticity assessed by a second sample of participants.

A first experiment based on this adaptation of the lawyer-engineer problem, compared three groups of participants. One group estimated $p(H \mid D)_{\text {High }}$ where $p(H)=$ $.70, D=\mathrm{d}_{i}$ for $i=1$ to 5 and $\mathrm{d}_{1}, \ldots, \mathrm{d}_{5}$ denoted five different thumbnail descriptions; another group estimated $p(H \mid D)_{\text {Low }}$ where $p(H)=.30$; a third group estimated the inverse probability $p(D \mid H)$, in the absence of base-rate information. The key objective of this experiment was to test the hypothesis that, within the Social-judgement paradigm, participants indeed commit the inverse fallacy; that is, that participants who are asked to estimate $p(H \mid D)$, whether $p(H)$ is high or low, are in fact estimating the inverse likelihood $p(D \mid H)$, the probability of observing the description $D$ when $H$ is already known to be true.

In a second experiment, participants were all asked to estimate $p(H \mid D), p(\sim H \mid D)$, $p(D \mid H), p(D \mid \sim H)$. They were also asked to evaluate the degree to which description $D$ was representative of categories $H$ and $\sim H$, respectively. The key objectives of this latter experiment were firstly to identify which of four predictor variables based on the inverse fallacy, representativeness, base-rate neglect, and Bayesian account, respectively, would best predict outcomes of posterior probability judgements. Secondly, the experiment was designed to test whether binary complementary posterior probability judgements were additive, and, thirdly, to test whether deviations from additivity could be inferred from corresponding inverse likelihood judgements. The next section of this chapter presents the methodology adopted to assure the diagnosticity of the thumbnail descriptions used. This is followed by the detailed presentation of each of the two experiments conducted and their respective results. The implications of those results for the process-focused study of posterior probability judgements within the Social-judgement paradigm are discussed in the final section of this chapter. 
CONSTRUCTION OF NEW THUMBNAIL DESCRIPTIONS

\section{Elicitation of typical traits and hobbies}

Recent findings have suggested that the personality sketches used by Kahneman and Tversky (1973) might be a bit obsolete and lack diagnosticity (e.g., Fischhoff \& BarHillel, 1984; Politzer \& Macchi, 2000). As a consequence, a pilot study was carried out in order to construct typical and up-to-date thumbnail descriptions. First, participants $(N=15)$ were questioned individually, using a single-screen on-line questionnaire. They were told that the experimenter was "interested in culturally shared knowledge about typical instances" and that they will "simply be asked to recall such knowledge". Their task further read as follows:

Imagine that one of your friends tells you that he is invited to a reception for accountants and will need to make contact with them. You're having the following conversation on this topic:

- Friend: "I don't even know what an accountant is!"

- You: "Well, accountants are persons trained to keep accounts..."

- Friend: "Yes, but what are they like?"

Imagine you are answering your friend this last question by enumerating 5 traits (e.g., open-minded, serious, etc.) that could be used to describe someone who is an accountant.

Below, a form was presented, and participants were prompted to 'enumerate five traits that one could use to describe someone who is an accountant'. The scenario was then continued:

Friend: "I see! Now, given that I don't know anything about accountancy, what else would accountants be interested in? What kind of more general interests or hobbies do you think they would be likely to have?"

In another subsequent form, participants were prompted to "list five hobbies or general interests that one could use to describe someone who is an accountant". Finally, an isomorphic task albeit concerned with the description of designers was introduced and participants were asked to list five traits and five hobbies that could be used to describe someone who is a designer. The sample of traits collected is presented in Table IV-1. 
Table IV-1

Stereotypical traits and hobbies/interests attributed to accountants and designers by 15 judges.

\begin{tabular}{|c|c|}
\hline Stereotypical traits & Hobbies/interests \\
\hline \multicolumn{2}{|c|}{ Accountants } \\
\hline $\begin{array}{l}\text { Analytic, logical, methodical, organized, } \\
\text { pragmatic, responsible, sensible, systematic, } \\
\text { tidy (14). }\end{array}$ & $\begin{array}{l}\text { Current affairs, economy, news, politics, } \\
\text { share dealing, tax raisesldecreases (15). } \\
\text { Golf, sailing, sports, squash, swimming (14). }\end{array}$ \\
\hline Honest, serious, trustworthy (13). & Brain teasers, chess, computing, crosswords, \\
\hline $\begin{array}{l}\text { Conscientious, detailed, fastidious, hard- } \\
\text { working, meticulous, particular, precise (11). }\end{array}$ & $\begin{array}{l}\text { general knowledge, home improvement, } \\
\text { reading, stamp collecting (13). }\end{array}$ \\
\hline \multirow{2}{*}{$\begin{array}{l}\text { Mathematical, numerate (8). } \\
\text { Educated, intelligent, knowledgeable, } \\
\text { professional, skilled (6). Boring, dull (4). } \\
\text { Committed, dedicated (2). Greedy, stingy } \\
\text { (2). Stubborn (2). }\end{array}$} & Cinema, classical music, football, movies, \\
\hline & $\begin{array}{l}\text { music, TV (11). } \\
\text { Country walks, pub lunches, pubs, quiet } \\
\text { holidays, socializing (6). }\end{array}$ \\
\hline $\begin{array}{l}\text { (2). Stubborn (2). } \\
\text { Various: conservative, discreet, immoral, } \\
\text { limited, materialistic, not talkative, sneaky, } \\
\text { ugly, unfashionable, uptight, wears glasses. }\end{array}$ & $\begin{array}{l}\text { Various: management, multimedia, theatre, } \\
\text { train- spotting. }\end{array}$ \\
\hline
\end{tabular}

Designers

Artistic, creative, imaginative, innovative, Art, drawing, painting, multimedia, (20). original (19).

Extroverted, freethinking, gregarious, openminded, outgoing (11).

Ambitious, flamboyant, image conscious, loud, pretentious (6). Cool, fashionable, in, popular, well dressed (5). Fun-loving, funny, party lover (4). Intelligent, skilled (2). Dreamer (2). Eccentric (2). Sensitive (2).

Fancy restaurants, parties, socializing (11).

Sunbathing, travel (9).

Music, musicals, opera, theatre (7).

Fashion, manicures, magazines, clothes shopping (6). Cycling, jogging, sports (5).

Various: architecture, current affairs, playing twister, sex, strip cartoons.

Various: big spender, confident, cultured, dynamic, emotional, enthusiastic, hard working, hyperactive, insouciant, interesting, lonely, mad, opinionated, outspoken, selfish, stressed, talkative.

Note. The numbers in brackets indicate the frequencies of occurrence, the traits and hobbies are semantically grouped.

\section{Construction and assessment of the descriptions' typicality}

Five short descriptions were constructed on the basis of the data collected in the first part of this study. Each description was made of eight different traits and hobbies. The respective proportions of designer and accountant traits and hobbies were varied so that the degree to which each of the five descriptions was representative of a designer or an accountant, would differ for each description. The ratio of the number of designer traits and hobbies to that of accountant traits and hobbies were respectively 8:0, 5:3, 4:4, 3:5 and 0:8 with the aim to create a typical designer, designer, neutral, accountant and typical 
accountant description, respectively. The resulting verbal descriptions are presented in Table IV-2.

Table IV-2

Thumbnail descriptions constructed from a gathered sample of typical traits.

\begin{tabular}{|c|c|}
\hline Type & Description \\
\hline $\mathrm{d}_{1}$ (typical designer) & $\begin{array}{l}\text { 'Creative, open-minded and outgoing. Quite ambitious. Knows all } \\
\text { about new multimedia technologies. Loves to socialise and often } \\
\text { have dinner in fancy restaurants. Spent his last holiday surfing in } \\
\text { Hawaii.' }\end{array}$ \\
\hline $\mathrm{d}_{2}$ (designer) & $\begin{array}{l}\text { 'Has artistic talents and is very imaginative. Regularly goes to } \\
\text { comedy shows. Buys fashion magazines and is quite image } \\
\text { conscious. Likes to read historical novels and do crosswords. Is quite } \\
\text { numerate.' }\end{array}$ \\
\hline $\mathrm{d}_{3}$ (neutral) & $\begin{array}{l}\text { 'Is innovative, meticulous and quite a knowledgeable person. Is a } \\
\text { party lover and has a lot of friends. Likes to read about current } \\
\text { affairs and is a very good chess player. Always wear fashionable } \\
\text { clothes.' }\end{array}$ \\
\hline $\mathrm{d}_{4}$ (accountant) & $\begin{array}{l}\text { 'Quite logical and has an analytical mind. Enjoys quiet family } \\
\text { holidays, Sunday pub lunches and country walks. Regularly goes for } \\
\text { a run. Is sometimes dreamy and loves to do home crafts. }\end{array}$ \\
\hline $\mathrm{d}_{5}$ (typical accountant) & $\begin{array}{l}\text { 'Generally serious, } \underline{\text { hard-working and well organised. Shows great }} \\
\text { interest in political issues and share dealing. Likes to play golf and } \\
\text { listen to classical music. Possesses an impressive stamp collection.' }\end{array}$ \\
\hline
\end{tabular}

Note. Each description was made with eight different traits taken from a sample of previously collected traits. The proportion of designer vs. accountant traits was varied for each description. Underlined traits are traits provided to describe an accountant.

The diagnosticity of these descriptions was evaluated independently. A total of 20 University of Hertfordshire students volunteered to participate in the second part of this pilot study. They were tested individually with a seven-screen on-line questionnaire. The first screen presented their task as follows:

A panel of psychologists have interviewed and administrated personality tests to designers and accountants, all successful in their respective fields. On the basis of this information, thumbnail descriptions have been written. You will be presented with five descriptions. Your task will be to rate the typicality of each of these descriptions.

Then the participants were instructed to click on a button to continue. Table IV-2 presents the five sketches used in the study. The next five screens presented these sketches in a randomised order. For each sketch, participants were reminded that the description corresponded to either a designer or to an accountant and were asked to rate the typicality of the description by choosing between five alternatives, as indicated in 
Figure IV-1. Participants' ratings were attributed a score between -2 (undoubtedly accountant) and +2 (undoubtedly designer).

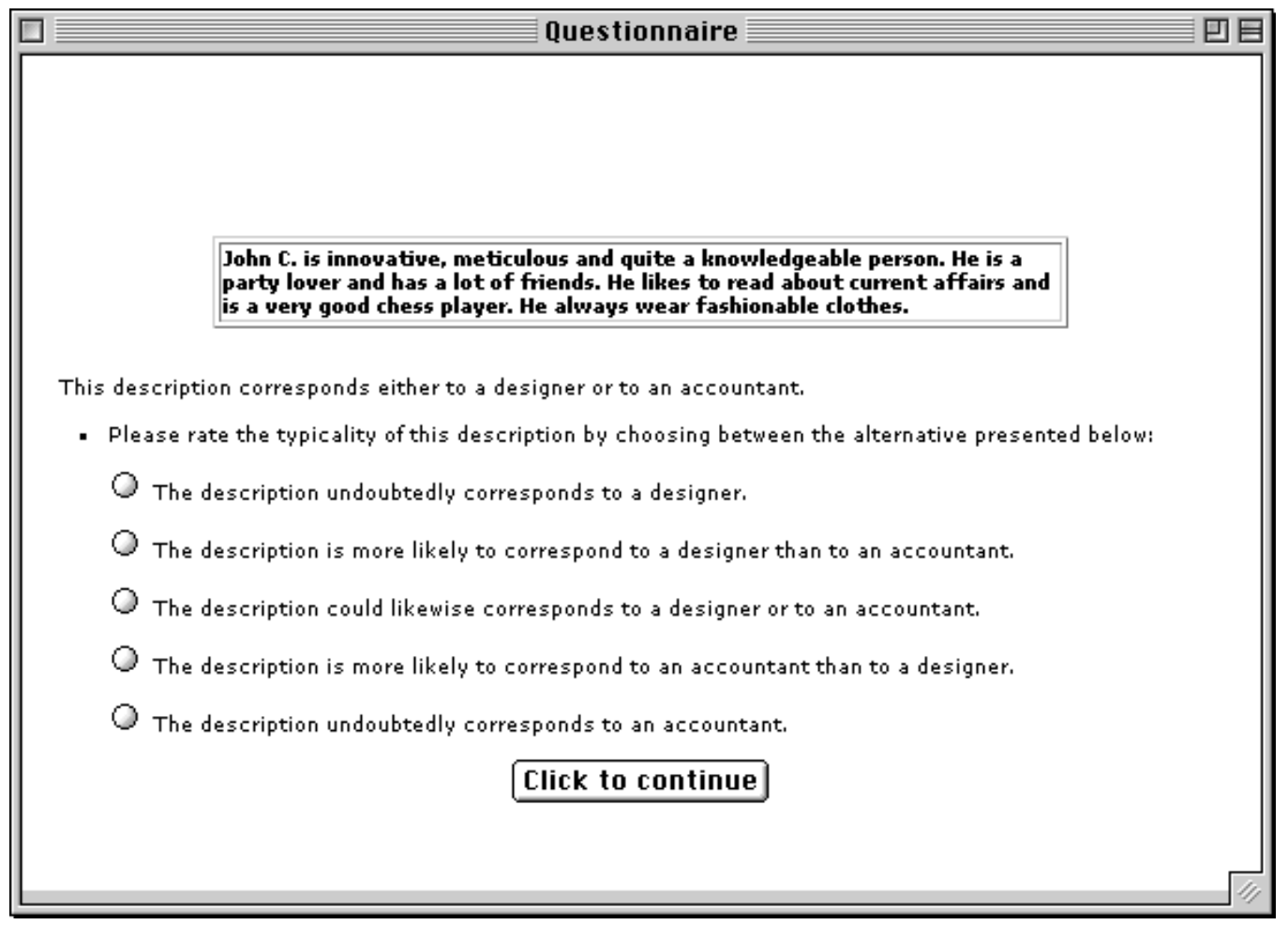

Figure IV-1 - Display of the pilot questionnaire presented to check the diagnosticity of the five thumbnail descriptions.

Each of the 5 personality descriptions represented a unique stimulus condition in a 5(Typicality: undoubtedly-designer, designer, neutral, accountant, undoubtedlyaccountant) repeated-measure design with the rating score as the dependent variable measured for each stimulus. Figure IV-2 presents the mean ratings for each description. 


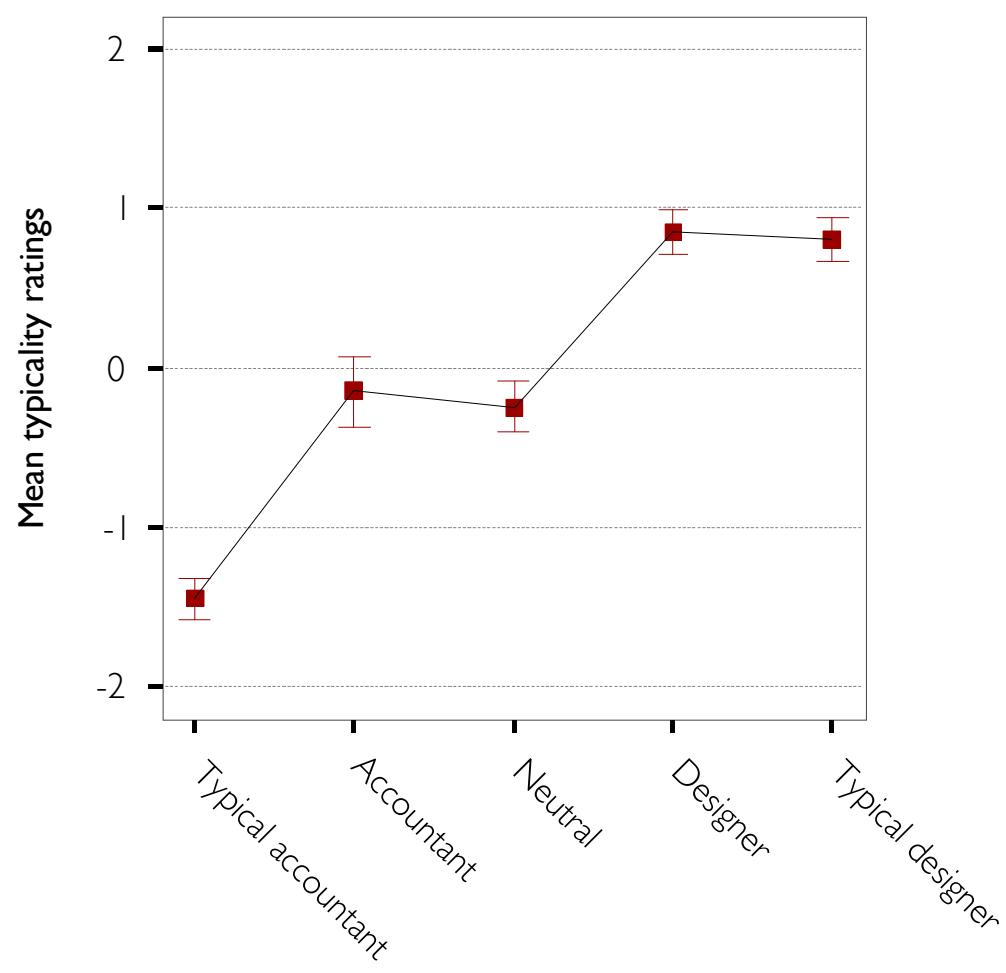

Descriptions

Figure IV-2 - Mean typicality ratings as a function of the description presented, with standard errors.

Note. Participants' ratings were attributed a score between -2 ('undoubtedly accountant') and +2 ('undoubtedly designer').

Planned paired samples t-tests were carried out to check that the differences in mean scores observed were statistically significant at the $.0125 \alpha$-level (to protect against an inflated type I error). These tests revealed that the typicality score for the "typical accountant" description $[\mathrm{M}=-1.45, \mathrm{SD}=.61]$ was significantly lower than that for the “accountant" description $[M=-0.15, S D=.99 ; t(19)=-8.85, p<.0001]$. Neither the "accountant" and "neutral" descriptions $[\mathrm{M}=-0.25, \mathrm{SD}=.72]$, nor the "designer" $[\mathrm{M}$ $=0.85 ; \mathrm{SD}=.59]$ and "typical designer" $[\mathrm{M}=0.80 ; \mathrm{SD}=.62]$ descriptions significantly differ from each other $[t(19)=.34$ and $t(19)=1.00$, respectively, $p>.30]$. Typicality scores for the "neutral" and the "designer" descriptions, however, did differ significantly $[t(19)=-6.85, p<.0001]$. An analysis of trend revealed the presence of a strong linear trend $\left[F(1,19)=85.15, p<0.0001\right.$, partial $\eta^{2}=.82$, MSE $\left.=.71\right]$. Although the typicality ratings were not exactly as expected (i.e., there was only one description judged as representing an accountant $\left(\mathrm{d}_{5}=\right.$ 'typical accountant'), two neutral descriptions ( $\mathrm{d}_{3}=$ 'neutral' and $\mathrm{d}_{4}=$ 'accountant') and two designer descriptions ( $\mathrm{d}_{1}=$ 'typical designer' and $\mathrm{d}_{2}=$ 'designer'), the descriptions nevertheless 
elicited different typicality levels and all descriptions were therefore kept unchanged in the experimental material.

\section{EXPERIMENT 1}

This experiment was aimed at examining whether the inverse fallacy could explain the results observed in the Social-judgement paradigm. A problem adapted from Kahneman and Tversky's (1973) lawyer-engineer study was used, based on the materials described above. Some participants were asked to make posterior probability judgements, others were asked to make inverse likelihood judgements. The first hypothesis was that a manipulation of the base rates would have little effect on posterior probability judgements. The second hypothesis was that observed judgements would significantly differ from their Bayesian counterparts. Finally, the main hypothesis of this experiment was that observed posterior probability judgements would be confounded with judgements of inverse likelihood, thereby suggesting that committing the inverse fallacy could account for posterior probability judgements.

\section{Method}

\section{Participants}

An opportunity sample of 48 University of Hertfordshire students recruited on campus (32 women and 16 men, mean age $=22.8$ years, age range $=18-35$ ), volunteered to participate in this experiment. They were tested in small groups.

\section{Design}

A between-subjects design was used. The first factor (description order) had two levels (order 1 and order 2) and the second factor (judgement type) had 3 levels $\left[p(H \mid D)_{\text {High }}, p(H \mid D)_{\text {Low }}\right.$, and $\left.p(D \mid H)\right]$. There were 5 dependent measures, namely, probability judgements for the typical designer $\left(d_{1}\right)$, the designer $\left(d_{2}\right)$, the neutral $\left(d_{3}\right)$,

the accountant $\left(\mathrm{d}_{4}\right)$, and the typical accountant $\left(\mathrm{d}_{5}\right)$ descriptions. Traditionally, this would have been viewed as a within-subjects factor with 5 levels corresponding to the five descriptions used. Yet, the effect of the different descriptions on judgements was irrelevant to the purpose of this experiment. Indeed this experiment was not designed to test whether two identical types of posterior probability judgements would differ as a function of their conditioning description [e.g., whether $p\left(H \mid d_{1}\right)_{\text {High }}$ would differ from $\left.p\left(H \mid d_{2}\right)_{\text {High }}\right]$. Instead, for any given description, the purpose of this experiment was to 
test whether different types of posterior probability judgements would differ [e.g., whether $p\left(H \mid d_{1}\right)_{\text {High }}, p\left(H \mid d_{1}\right)_{\text {Low }}$ and $p\left(d_{1} \mid H\right)$ would differ from each other]. Therefore, judgements for each of the five descriptions were treated as separate measures. Participants were assigned randomly to one of the six groups resulting from the combination of the two experimental factors used.

According to the first hypothesis, $p(H \mid D)_{\text {High }}$ and $p(H \mid D)_{\text {Low }}$ were not expected to differ. The second hypothesis suggested that $p(H \mid D)_{\text {High }}$ judgements would significantly differ from their Bayesian counterparts, estimated from $p(H \mid D)_{\text {Low }}$ judgements. Similarly, $p(H \mid D)_{\text {Low }}$ judgements were expected to be significantly different from their Bayesian counterparts, this time estimated from the knowledge of $p(H \mid D)_{\text {High }}$ judgements. Finally, the last hypothesis suggested that both $p(H \mid D)_{\text {High }}$ and $p(H \mid D)_{\text {Low }}$ judgements would not significantly differ from $p(D \mid H)$ judgements.

\section{Materials and procedure}

The experiment consisted of three experimental conditions. The first two conditions, the "high base-rate" and "low base-rate" conditions, were designed to replicate Kahneman and Tversky's (1973) findings. Participants in the high base-rate condition were presented with the following 2-page questionnaire:

A panel of psychologists have interviewed and administered personality tests to 70 designers and 30 accountants, all successful in their respective fields. On the basis of this information, thumbnail descriptions of the 70 designers and the 30 accountants have been written. A bug in the computer storing the data resulted in the loss of the information concerning the profession of the person described. Five descriptions, chosen at random from the 100 available descriptions, will be presented to you. For each description, please indicate your probability that the person described is a designer, on a scale from 0 to 100.

The scenario in the low base-rate condition was identical, apart from the proportions of designers and accountants, respectively set to .30 and .70 . This introduction was followed by the presentation of five individual descriptions, based on the personality descriptions presented in Table IV-2. For each description, participants were asked to estimate the chances that the person described was one of the designers, on the basis of the description presented [i.e., $p(H \mid D)_{\text {High }}$ and $p(H \mid D)_{\text {Low }}$ in the high and low base-rate conditions, respectively, where $H$ was arbitrarily defined as the designer category]. The structure of this questionnaire slightly differed from that of Kahneman and Tversky (1973). First, the information concerning the computer bug was added so that the need for estimating posterior probabilities was justified. Secondly, participants were explicitly asked to estimate the chances that the person was a designer, given his or her description 
to avoid ambiguity. For instance, the "typical designer" description in the high base-rate condition read as follows:

Andrew G. is creative, open-minded and outgoing. He is quite ambitious. He knows all about new multimedia technologies. He loves to socialize and often have dinner in fancy restaurants. He spent his last holiday surfing in Hawaii.

Given his description, what is the chance that Andrew is one of the 70 designers? There is a ___ \% chance.

The description in the low base-rate condition was identical, except that the end of the question read “...that Andrew is one of the 30 designers?" Two randomised orders of description presentation were used (i.e., order $_{1}=\{$ typical designer, accountant, typical accountant, designer, neutral $\}$, and order $_{2}=$ typical accountant, neutral, accountant, designer, typical designer\}). It was hypothesised that although judgements would vary as a function of the description on which they were based, the manipulation of base-rates would have little effect on the judgements.

In the last experimental condition, the introduction was similar to the two previous conditions except that no base-rate information was provided to participants who were then asked to evaluate the inverse likelihood $p(D \mid H)$. Participants in this condition read the following introduction:

A panel of psychologists have interviewed and administered personality tests to professionals, all successful in their respective fields. On the basis of this information, thumbnail descriptions have been written. A bug in the computer storing the data resulted in the loss of the information concerning the profession of the person described. Five descriptions, chosen at random from 100 available descriptions, will be presented to you. For each description, please indicate your probability that a person who is a designer can be portrayed by the description at hand on a scale from 0 to 100.

Next, the five descriptions were presented in either one of the two randomised orders specified above. This time, however, the descriptions were presented in a generic form. For example, the "typical designer" description read,

Creative, open-minded and outgoing. Quite ambitious. Knows all about new multimedia technologies. Loves to socialise and often have dinner in fancy restaurants. Spent his last holiday surfing in Hawaii.

\section{Results}

\section{Evaluation of normality assumption}

To warrant the use of parametric statistical tests, the normality of the distributions of $p(H \mid D)_{\text {High }}, p(H \mid D)_{\text {Low }}$ and $p(D \mid H)$ judgements for each of the five descriptions 
presented was assessed by examining skewness and kurtosis statistics. Tabachnick and Fidell (1996) recommended computing the $z$ scores for each of these statistics and indicated that their significance could be assessed by using a .001 two-tailed alpha level. By these criteria, no significant departure from normality was observed (i.e., all ps $>$ .001). Consequently, parametric statistical tests were used throughout.

\section{Order effect}

To test for description order effects, the data were subjected to a multivariate analysis of variance (MANOVA) with two between-subjects factors. The first factor (description order) had two levels (order 1, order 2). The second factor (judgement type) had three levels $\left[p(H \mid D)_{\text {High }}, p(H \mid D)_{\text {Low }}, p(D \mid H)\right]$. The judgements observed for each of the five descriptions were used as dependent measures. Results showed that the order of presentation of the descriptions did not significantly affect judgements [Multivariate $F(5,38)=0.71$, Wilk's $\Lambda=0.91, p=.62$, partial $\eta^{2}=0.09$ ] nor did it significantly interact with the judgement type factor $(p=.38)$. Data were therefore collapsed across orders.

\section{Extent of base-rate usage}

The first aim of this study was to check that Kahneman and Tversky's (1973) results were replicated with the present problem. In order words, it was expected that the manipulation of base rates would have little effect on the posterior probability judgements observed. In line with this analysis, Figure IV-3 suggests that overall, the base-rate manipulation did not affect judgements as much as Bayes's theorem would warrant since pairs of medians were closer to the identity line than to the Bayesian line. 


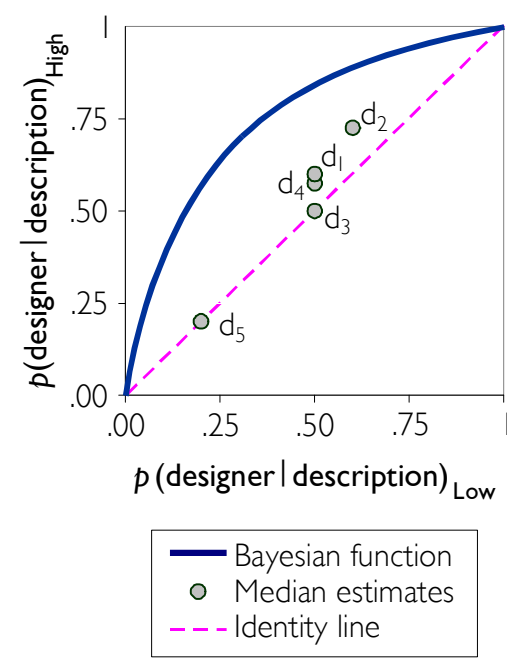

Figure IV-3 - Plot of the median posterior probability judgements observed in the high base-rate condition against those observed in the low base-rate condition.

Note. $\mathrm{d}_{1}, \mathrm{~d}_{2}, \mathrm{~d}_{3}, \mathrm{~d}_{4}$, and $\mathrm{d}_{5}$ refer to the typical designer, designer, neutral, accountant and typical accountant descriptions respectively, as presented in Table IV-2.

To test for base-rate effect, data from the first two groups of participants were subjected to a Multivariate Analysis of Variance (MANOVA) with one betweensubjects factor (category base rate) with two levels (high, low). The judgements for each of the five descriptions represented the dependent measures. Overall, the base-rate manipulation did not significantly affect judgements [Multivariate $F(5,26)=1.071$, Wilk's $\Lambda=0.83, p=.40$, partial $\left.\eta^{2}=0.17\right]$.

Following Wells and Harvey's (1978), Gigerenzer et al.'s (1988) and Baratgin and Noveck's (2000) analyses, base rate usage was further evaluated on the basis of the difference between posterior probability judgements in the high and low base-rate conditions. The first two columns of Table IV-3 present the mean obtained in each condition for each of the five descriptions presented. The third column presents the difference between mean judgements in the high and low base-rate condition, respectively, thereby indicating the extent to which judgements were affected by the base-rate manipulation. 
Table IV-3

Comparison of mean judgements observed in high and low base-rate conditions.

\begin{tabular}{|c|c|c|c|c|c|c|}
\hline \multirow[b]{3}{*}{$D$} & \multicolumn{2}{|c|}{ Base-rate condition } & \multirow[b]{3}{*}{$\Delta_{\mathrm{M}}$} & \multirow[b]{3}{*}{$F(1,30)$} & \multirow[b]{3}{*}{ MSE } & \multirow[b]{3}{*}{ Partial $\eta^{2}$} \\
\hline & High & Low & & & & \\
\hline & M & M & & & & \\
\hline $\mathrm{d}_{1}$ & .62 & .52 & .10 & $1.60 \mathrm{~ns}$ & 0.04 & 0.05 \\
\hline $\mathrm{d}_{2}$ & .74 & .66 & .08 & $1.17 \mathrm{~ns}$ & 0.04 & 0.04 \\
\hline $\mathrm{d}_{3}$ & .44 & .52 & -.08 & $0.63 \mathrm{~ns}$ & 0.08 & 0.02 \\
\hline $\mathrm{d}_{4}$ & .49 & .44 & .05 & $0.34 \mathrm{~ns}$ & 0.07 & 0.01 \\
\hline $\mathrm{d}_{5}$ & .32 & .28 & .04 & $0.14 \mathrm{~ns}$ & 0.07 & 0.00 \\
\hline M & .52 & .48 & .04 & & & \\
\hline
\end{tabular}

Note. $\mathrm{d}_{1}, \mathrm{~d}_{2}, \mathrm{~d}_{3}, \mathrm{~d}_{4}$, and $\mathrm{d}_{5}$ refer to the typical designer, designer, neutral, accountant and typical accountant descriptions respectively, as presented in Table IV-2.

Partial $\eta^{2}=\frac{S S_{\text {effect }}}{S S_{\text {effect }}+S S_{\text {error }}}$.

This table shows that the difference between posterior probability judgements in high and low base-rate conditions were generally in the right direction. Namely, the differences between $p(H \mid D)_{\text {High }}$ and $p(H \mid D)_{\text {Low }}$ judgements were always positive, with the exception of judgements conditioned on description $\mathrm{d}_{3}$. Yet, the sizes of the effect of base-rate for each of the descriptions used were minimal, and the differences observed were never statistically significant.

\section{Analysis of judgements against Bayesian benchmarks}

The second hypothesis tested was that posterior probability judgements would significantly differ from Bayesian prescriptions. As already noted in chapter III, the Bayesian value of $p(H \mid D)_{\text {Low }}$ can be inferred from the judgement of $p(H \mid D)_{\text {High }}$ whereas the Bayesian value of $p(H \mid D)_{\text {High }}$ can be inferred from the judgement of $p(H \mid D)_{\text {Low }}$. Accordingly, for each individual judgement observed in the high base-rate condition, a corresponding Bayesian value for low base-rate was computed using equation III.1 (see p. 42). Similarly, for each individual judgement observed in the low base-rate condition, a corresponding Bayesian value for high base-rate was computed using equation III.3 (see p. 42). Normality tests revealed that expected Bayesian values were normally distributed, except for the 'neutral' description $\left(\mathrm{d}_{3}\right)$, which led to a distribution with statistically significant negative skewness (skewness $=-1.91, z=3.39, p=0.0007$ ) in the high base rate condition, and the 'typical accountant' description $\left(\mathrm{d}_{5}\right)$, which led to 
a distribution with statistically signitificant positive skewness (skewness $=1.92, z=$ $3.40, p=0.0007$ ) in the low base rate condition. The data generated by $\mathrm{d}_{3}$ in the high base rate condition, and by $\mathrm{d}_{5}$ in the low base-rate condition were consequently discarded and parametric tests were applied using measures based on $d_{1}, d_{2}, d_{4}$, and $d_{5}$ in the high base-rate condition and based on $\mathrm{d}_{1}, \mathrm{~d}_{2}, \mathrm{~d}_{3}$, and $\mathrm{d}_{4}$ in the low base-rate condition.

In Figure IV-4 the mean Bayesian values are plotted against the observed mean posterior probability judgements in both high and low base-rate conditions, for each of the four descriptions used.

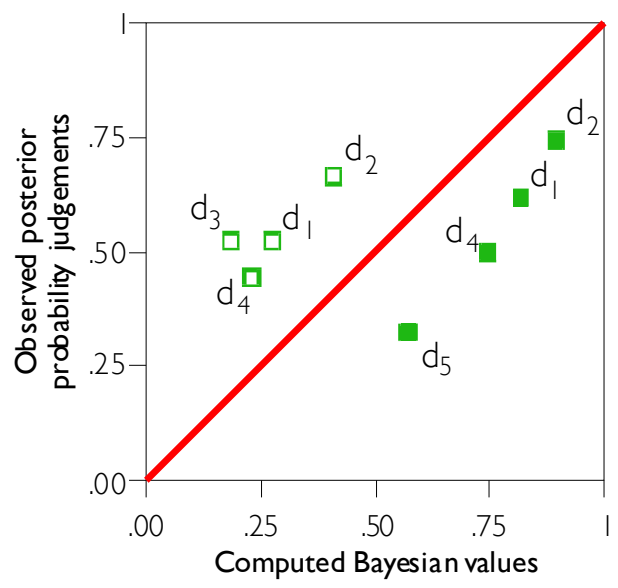

\begin{tabular}{|ll|}
\hline$\square$ & High base rate \\
$\square \quad$ & Low base rate \\
$\square$ & Id entity \\
\hline
\end{tabular}

Figure IV-4 - Plot of the mean posterior probability judgements observed against the mean Bayesian values computed for the high and low base-rate condition.

Note. $\mathrm{d}_{1}, \mathrm{~d}_{2}, \mathrm{~d}_{3}, \mathrm{~d}_{4}$, and $\mathrm{d}_{5}$ refer to the typical designer, designer, neutral, accountant and typical accountant descriptions respectively, as presented in Table IV-2.

If observed judgements were Bayesian on average, the data points would have fallen on the identity line. In contrast, Figure IV -4 reveals that judgements generally departed from the Bayesian norm. To test whether the observed judgements in the high base-rate condition significantly differed from expected Bayesian values, which were inferred from judgements observed in the low base-rate condition, these data [i.e., $p(H \mid D)_{\text {High }}$ judgements observed and $p(H \mid D)_{\text {High }}$ Bayesian computed values, for $i=1,2,4$, and 5] were subjected to a Multivariate Analysis of Variance (MANOVA) with one betweensubjects factor (judgement) with two levels (observed, computed). The measures for each of the descriptions $d_{1}, d_{2}, d_{4}$, and $d_{5}$ represented the dependent measures. Similarly, 
to test whether $p(H \mid D)_{\text {Low }}$ judgements significantly differ from expected Bayesian values under low base-rates, measures for the descriptions $\mathrm{d}_{1}, \mathrm{~d}_{2}, \mathrm{~d}_{3}$, and $\mathrm{d}_{4}$ were also subjected to the same MANOVA. Results show that judgements significantly differed from expected Bayesian values both in the high base-rate condition and in the low base-rate condition [Multivariate $F(4,27)=9.75$, Wilk's $\Lambda=0.41, p<.0001$, partial $\eta^{2}=0.59$ and Multivariate $F(4,27)=6.44$, Wilk's $\Lambda=0.51, p<.001$, partial $\eta^{2}=0.49$, respectively].

To identify the dependent measures that contributed to this effect, three further comparisons for each description were conducted with an adjusted $\alpha$-level of .0125 , to warrant the analysis against an inflated Type I error. These planned analyses are summarised in Table IV-4. Results revealed that all mean judgements significantly differed from Bayesian prescriptions, with the exception of judgements made on the basis of description $\mathrm{d}_{1}$ in the high base rate condition.

Table IV-4

Comparison of mean judgements observed in high and low base-rate conditions with their Bayesian counterparts.

\begin{tabular}{|c|c|c|c|c|c|c|}
\hline$D$ & Observed & Bayesian & $\Delta_{M}$ & $F(1,30)$ & MSE & Partial $\eta^{2}$ \\
\hline \multicolumn{7}{|c|}{ High base rates } \\
\hline $\mathrm{d}_{1}$ & .62 & .58 & .04 & $0.26 \mathrm{~ns}$ & 0.05 & 0.01 \\
\hline $\mathrm{d}_{2}$ & .74 & .89 & -.16 & $10.06^{*}$ & 0.02 & 0.25 \\
\hline $\mathrm{d}_{4}$ & .49 & .75 & -.26 & $7.71^{*}$ & 0.07 & 0.20 \\
\hline $\mathrm{d}_{5}$ & .32 & .82 & -.50 & $38.58^{*}$ & 0.05 & 0.56 \\
\hline M & .54 & .76 &.-22 & & & \\
\hline \multicolumn{7}{|c|}{ Low base rates } \\
\hline $\mathrm{d}_{1}$ & .52 & .28 & .25 & $10.97^{*}$ & 0.04 & 0.27 \\
\hline $\mathrm{d}_{2}$ & .66 & .41 & .25 & $9.92^{*}$ & 0.05 & 0.25 \\
\hline $\mathrm{d}_{3}$ & .52 & .19 & .33 & $7.26^{*}$ & 0.05 & 0.35 \\
\hline $\mathrm{d}_{4}$ & .44 & .23 & .20 & $16.16^{*}$ & 0.05 & 0.19 \\
\hline $\mathrm{M}$ & .53 & .28 & .26 & & & \\
\hline \multicolumn{7}{|c|}{${ }^{*} p<.0125 ;$ ns $=$ not significant. } \\
\hline \multicolumn{7}{|c|}{$\begin{array}{l}\text { Note. } \mathrm{d}_{1}, \mathrm{~d}_{2}, \mathrm{~d}_{3}, \mathrm{~d}_{4} \text {, and } \mathrm{d}_{5} \text { refer to the typical designer, designer, neutral, } \\
\text { accountant and typical accountant descriptions respectively, as presented } \\
\text { in Table IV-2. Partial } \eta^{2}=\frac{S S_{\text {effect }}}{C S} \text {. }\end{array}$} \\
\hline
\end{tabular}




\section{Inverse fallacy and posterior probability judgements}

Kahneman and Tversky (1973) proposed that participants relied on the representativeness heuristic when making judgements in the lawyer-engineer problem. The concept of representativeness was later criticised for its lack of a clear-cut definition. The main aim of the present study was to test the hypothesis that judgements of representativeness can be conceptualised as inverse likelihood judgements. In Figure IV -5, the mean $p(H \mid D)$ judgements observed in both the high base-rate and low baserate groups are plotted against the mean judgements observed in the inverse likelihood group, for each of the five descriptions used.

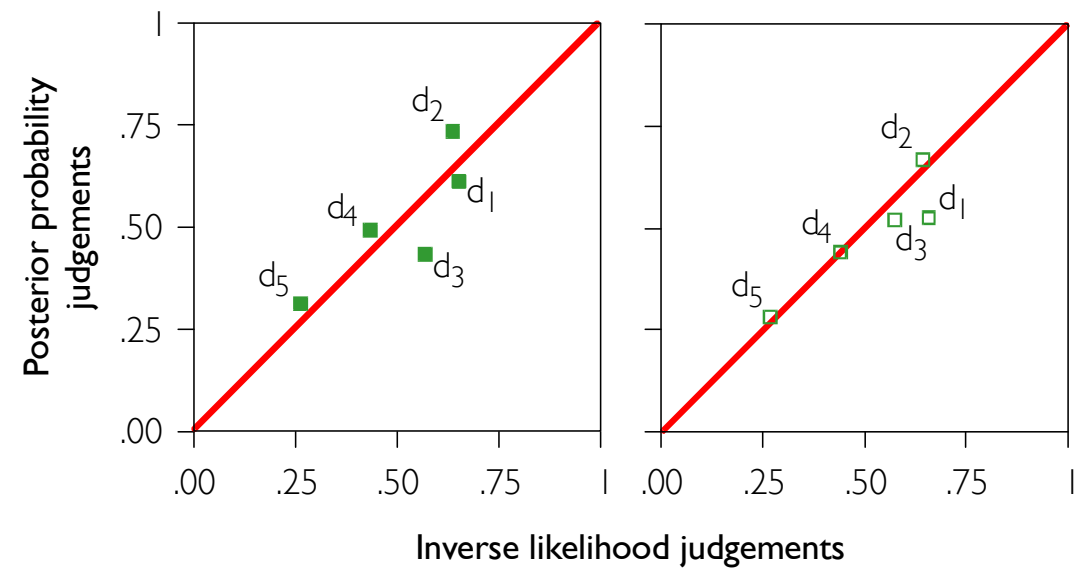

\begin{tabular}{|ll|}
\hline$\square$ & High base rate \\
$\square \quad$ & Low base rate \\
Identity
\end{tabular}

Figure IV-5 - Plots of the mean posterior probability judgements observed against the inverse likelihood judgements for the high and low base-rate condition.

Note. $\mathrm{d}_{1}, \mathrm{~d}_{2}, \mathrm{~d}_{3}, \mathrm{~d}_{4}$, and $\mathrm{d}_{5}$ refer to the typical designer, designer, neutral, accountant and typical accountant descriptions respectively, as presented in Table IV-2.

Figure IV-5 reveals that, on average, posterior probability judgements of $p(H \mid D)$ in either high or low base-rate conditions varied as a function of the description presented, but were remarkably close to corresponding judgements of the inverse likelihood $p(D \mid H)$, for each of the five description presented, as indicated by the falling of their coordinates either on or close to the identity line.

To test whether posterior probability judgements in the high base-rate and low baserate condition significantly differ from inverse likelihood judgements, these data were subjected to a Multivariate Analysis of Variance (MANOVA) with one between-subject factor (judgement type) with 3 levels (high base rate, low base rate, inverse likelihood) 
where the judgements for each of the five descriptions represented the dependent measures. Results show that overall judgements observed did not significantly differ as a function of the type of judgement elicited $\left[p(H \mid D)_{\text {High }}, p(H \mid D)_{\text {Low }}, p(D \mid H)\right]$ [Multivariate $F(10,82)=1.14$, Wilk's $\Lambda=0.77, p=.35$, partial $\eta^{2}=0.12$ ].

Regardless of the description provided, posterior probability judgements (whether in the high base-rate or low base-rate condition) were thus never significantly different from inverse likelihood judgements. These planned contrasts are summarised in Table IV-5.

Table IV-5

Simple contrasts of mean judgements observed in high and low base-rate conditions compared to inverse likelihood judgements, for each description.

\begin{tabular}{cccccccc}
\hline$D$ & $p(H \mid D)$ & $p(D \mid H)$ & $\Delta_{M}$ & $F(1,30)$ & MSE & Partial $\eta^{2}$ \\
\hline \multicolumn{7}{c}{ High base rates vs. inverse likelihood judgements } \\
$\mathrm{d}_{1}$ & .62 & .66 & -.05 & $0.49 \mathrm{~ns}$ & 0.03 & 0.02 \\
$\mathrm{~d}_{2}$ & .74 & .65 & .09 & $1.91 \mathrm{~ns}$ & 0.03 & 0.06 \\
$\mathrm{~d}_{3}$ & .44 & .58 & -.14 & $2.76 \mathrm{~ns}$ & 0.06 & 0.08 \\
$\mathrm{~d}_{4}$ & .49 & .44 & .05 & $0.33 \mathrm{~ns}$ & 0.07 & 0.01 \\
$\mathrm{~d}_{5}$ & .32 & .27 & .05 & $0.32 \mathrm{~ns}$ & 0.06 & 0.01 \\
$\mathrm{M}$ & .52 & .52 & .001 & & & \\
\hline
\end{tabular}

Low base rates

\begin{tabular}{lrrrrrl}
$\mathrm{d}_{1}$ & .52 & .66 & -.14 & $3.48 \mathrm{~ns}$ & 0.04 & 0.10 \\
$\mathrm{~d}_{2}$ & .66 & .65 & .02 & $0.05 \mathrm{~ns}$ & 0.04 & 0.002 \\
$\mathrm{~d}_{3}$ & .52 & .58 & -.06 & $0.56 \mathrm{~ns}$ & 0.05 & 0.02 \\
$\mathrm{~d}_{4}$ & .44 & .44 & -.004 & $0.00 \mathrm{~ns}$ & 0.04 & 0.0001 \\
$\mathrm{~d}_{5}$ & .28 & .27 & .01 & $0.03 \mathrm{~ns}$ & 0.04 & 0.001 \\
$\mathrm{M}$ & .48 & .52 & -.04 & & & \\
\hline
\end{tabular}

"p $<.01 ;$ ns $=$ not significant

Note. $\mathrm{d}_{1}, \mathrm{~d}_{2}, \mathrm{~d}_{3}, \mathrm{~d}_{4}$, and $\mathrm{d}_{5}$ refer to the typical designer, designer, neutral, accountant and typical accountant descriptions respectively, as presented in Table IV-2. Partial $\eta^{2}=\frac{S S_{\text {effect }}}{S S_{\text {effect }}+S S_{\text {error }}}$.

\section{Discussion of Experiment 1}

The objective of this first experiment was to replicate Kahneman and Tversky's (1973) findings that base-rate information appeared to be neglected when posterior probability judgements are made. Using a multivariate analysis of variance (MANOVA), the analysis of the results revealed that overall base-rate information did not significantly 
affect judgements. This result is inconsistent with the results originally observed by Kahneman and Tversky (1973) who reported that mean aggregate judgements (i.e., mean of judgements previously averaged over all five descriptions) in the high base-rate condition significantly differ from the same judgements in the low condition, even though this difference was relatively small $\left(\mathrm{M}_{\mathrm{H}}=.55\right.$ vs. $\mathrm{M}_{\mathrm{L}}=.50, \mathrm{~N}_{\mathrm{H}}=86, \mathrm{~N}_{\mathrm{L}}=85$; $t(169)=3.23, p<.01)$. A similar mean difference was observed in the present experiment (i.e., $\mathrm{M}_{\mathrm{H}}=.52$ vs. $\mathrm{M}_{\mathrm{L}}=.48, \mathrm{~N}_{\mathrm{H}}=16, \mathrm{~N}_{\mathrm{L}}=16$ ) but the same test conducted on the present set of data did not reach significance $[t(30)=0.71, p=.48]$. This inconsistency, however, can simply be explained in terms of difference in a priori power. Kahneman \& Tversky's (1973) result corresponds to effect size $d=0.49$ which gives an a priori power of .94 , given their sample size. ${ }^{1}$ The present experiment was based on a sample of 32 participants which gives an a priori power for detecting a similar effect size to that observed by Kahneman \& Tversky (1973) of only .38, but a power of .71 to detect a large effect size. Therefore, there might be a medium effect size for the base-rate factor but the present experiment had insufficient sample size to detect it. A minimum of 92 participants would have been required to achieve a priori power of .75 . The comparison with Bayesian benchmarks, however, does confirm that in any case base-rate utilisation was not optimal, as indicated by the validation of the second hypothesis tested, which proposed that judgements would significantly differ from Bayesian estimates.

The main hypothesis tested in this experiment was that $p(H \mid D)$ judgements observed were based on inverse likelihood estimates, i.e., estimates of $p(D \mid H)$. This hypothesis was supported in the sense that, on average, $p(H \mid D)$ judgements in either high or low base-rate condition were very close to inverse $p(D \mid H)$ judgements (see Figure IV-5). Statistical analyses confirmed that the mean posterior probability judgements (i.e., the mean of judgements previously averaged over all five descriptions) in either the high or the low base-base rate condition were not significantly different from mean inverse likelihood judgements [Univariate $F(2,45)=0.44, \mathrm{MSE}=0.02, p$ $=.65$, Partial $\left.\eta^{2}=0.02\right]$. The mean values of the judgements averaged over the five descriptions were $.52, .48$, and .52 in the high base-rate, low base-rate and likelihood conditions, respectively. With an estimated population standard deviation of 0.12 , in

\footnotetext{
${ }^{1}$ Where $\mathrm{d}=\frac{\mu_{1}-\mu_{2}}{s_{P}}, s_{P}=\frac{\mu_{1}-\mu_{2}}{t \sqrt{\frac{1}{N_{1}}+\frac{1}{N_{2}}}}$ and the power value is inferred using the GPOWER software.
} 
order to achieve a reasonable a priori power of .75 , one would require a total sample size of 438 participants. ${ }^{2}$ This indicates that the observed effect size is so small (effect size $f=$ 0.14) that one can safely conclude that it is negligible. One of the limitations of the present experiment, however, was that likelihood and posterior probability judgements were collected in different groups, resulting in a rather indirect test of the hypothesis that individuals evaluate a posterior probability on the basis of its inverse likelihood. Moreover, the low a priori power to detect a medium effect size might cast doubt on the capability of the sample size used to detect a significant difference. This shortcoming was addressed in Experiment 2, where both posterior and likelihood judgements were measured in a within-subjects design. In Experiment 1, a nomothetic research approach was followed; that is, aggregated judgements from groups of individuals were analysed in order to draw general conclusions about the computations underlying judgement. By contrast, an idiographic research approach was favoured in Experiment 2, where the research objective was to distinguish between the different strategies underlying judgement at an individual level to allow the test of the explanatory value of the inverse fallacy, in contrast with other possible judgemental computations and in relation with observed deviations from additivity.

\section{EXPERIMENT 2}

The objective of this experiment was to further investigate the descriptive value of the inverse fallacy, using a within-subjects design. The problem used in Experiment 1 was adapted for this purpose. Participants were asked to make posterior probability judgements and inverse likelihood judgements for both categories involved (i.e., $\mathrm{H}$ : 'designer' and $\sim H$ : 'accountant'), for the same five descriptions used in Experiment 1. Additionally, they were asked to evaluate the extent to which each description $\mathrm{d}_{i}$ was representative of categories $H$ and $\sim H$, respectively.

The first hypothesis tested was that posterior probability judgements would be best described by inverse likelihood judgements rather than by judgements of representativeness, computed Bayesian values, or even by "Base-Rate-Neglect-ButOtherwise-Bayesian" (BRNBOB) values. The second hypothesis concerned the additivity issue. Following Baratgin and Noveck (2000), it was hypothesized that the sum of posterior probability judgements for two collectively exhaustive and mutually

\footnotetext{
${ }^{2}$ Computed with the GPOWER statistical software.
} 
exclusive categories would not be additive. Finally, following from the assumption that individuals will commit the inverse fallacy, it was hypothesized that observed sums of posterior probability judgements will vary as a function of the sum of their respective inverse likelihood judgements.

\section{Method}

\section{Participants}

An opportunity sample of 16 University of Hertfordshire students (11 women, 4 men and 1 unspecified, mean age $=22.1$ years, age range $=18-33$ ) volunteered to participate in this experiment. They were recruited on campus and tested in small groups.

\section{Design}

A mixed design with three factors was used. The first factor (judgement type) was a within-subjects factor with three levels [posterior probability, inverse likelihood, representativeness]. The second factor (category order) was a between-subjects factor with two levels ('designer' first, 'accountant' first). The third factor (description order) was a between-subjects factor, also with two levels (order $1=\left\{\mathrm{d}_{1}, \mathrm{~d}_{4}, \mathrm{~d}_{5}, \mathrm{~d}_{2}, \mathrm{~d}_{3}\right\}$, order 2 $\left.=\left\{\mathrm{d}_{5}, \mathrm{~d}_{3}, \mathrm{~d}_{4}, \mathrm{~d}_{2}, \mathrm{~d}_{1}\right\}\right)$. As in Experiment 1, the different descriptions but also the two categories were not treated as within-subjects factors. This is because both the effect of these different descriptions and the effect of the two categories were not directly relevant to the purpose of the experiment. Therefore, judgements for the combinations of the different descriptions and the two categories were treated as separate measures. There were thus ten dependent measures (i.e., measures for each of five descriptions, namely, 'typical designer' $\left[\mathrm{d}_{1}\right]$, 'designer' $\left[\mathrm{d}_{2}\right]$, 'neutral' $\left[\mathrm{d}_{3}\right]$, 'accountant' $\left[\mathrm{d}_{4}\right]$, and 'typical accountant' $\left[\mathrm{d}_{5}\right]$ descriptions, and for target categories $H$ : 'designer' and $\sim H$ : 'accountant', respectively). Participants $(N=16)$ were assigned randomly to one of the four experimental groups and each participant made a total of 30 judgements.

The manipulation of orders was not expected to affect judgements. According to the first hypothesis, posterior and inverse likelihood judgements were expected to be closely and positively related whereas representativeness judgements, Bayesian or Base-RateNeglect-But-Otherwise-Bayesian values inferred from likelihood judgements were not expected to be as good predictors. According to the second hypothesis, complementary posterior probability judgements were expected to be non-additive (i.e., the sum of 
$p(H \mid D)$ and $p(\sim H \mid D)$ judgements was expected to be different from 1). Finally, according to the last hypothesis of this experiment, the sums of complementary posterior probability judgements [i.e., $p(H \mid D)+p(\sim H \mid D)$ ] were expected to be closely and positively related to the sums of their inverse likelihood judgements [i.e., $p(D \mid H)+$ $p(D \mid \sim H)]$.

\section{Materials and procedure}

All participants were presented with a 2-page questionnaire printed back-to-back on an A4 sheet in a landscape orientation. The cover story was identical to that used in Experiment 1, involving two professional categories, namely the designer and accountant categories. All participants were given the same numerical base rates [namely, $p(H)=.70$ and $p(\sim H)=.30$ ].

On the first page of the questionnaire, participants were instructed to read the introductory paragraph and were asked to make three types of judgements related to one of two categories, $H=$ "designer" or $\sim H=$ "accountant" (i.e., posterior, inverse and representativeness judgements) for each of the same five descriptions $d_{1}, d_{2}, d_{3}, d_{4}$, and $\mathrm{d}_{5}$ used in Experiment 1, which were printed on the right hand side of the page. The second page presented the same descriptions with a reminder of the instructions and the cover story and asked participants to provide the same three types of judgements but for the alternative target category. Eight participants were first asked to make judgements in relation to category $H$ ('designer'), and the remaining eight participants were first asked to make judgements in relation to category $\sim H$ ('accountant'). 


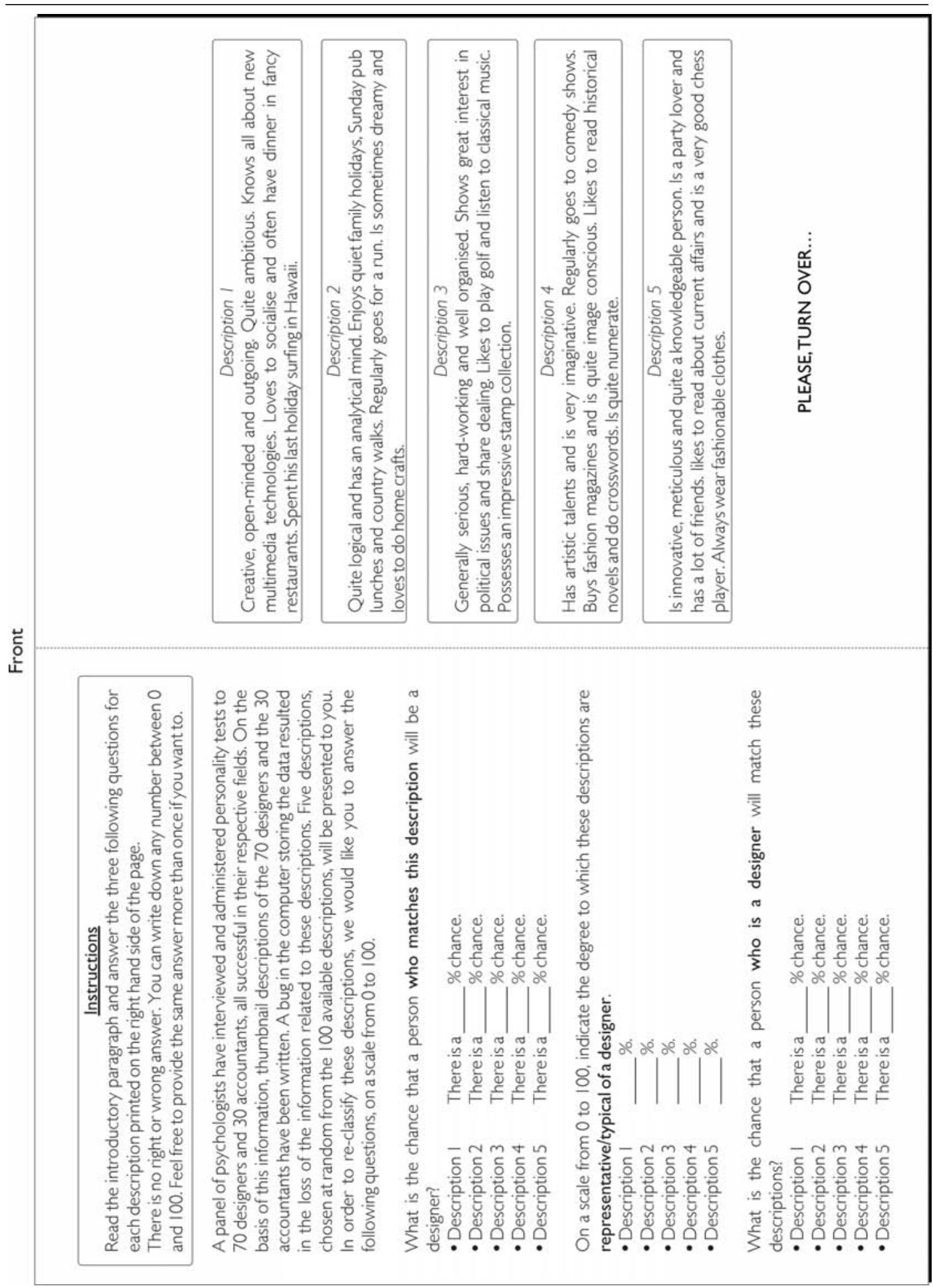

Figure IV-6 - Layout of the questionnaire used in Experiment 2 (description order 1, 'designer' measures).

Figure IV-6 illustrates the front layout of the questionnaire in the first of two orders of presentation of the descriptions and the first of two orders of target category [i.e., where judgements are first measured for the category $H$ ('designer') and then for the category $\sim H($ ('accountant') ]. 
The questions 'What is the chance that a person who matches this description will be a designer?', 'On a scale from 0 to 100, indicate the degree to which these descriptions are representative/typical of a designer', and 'What is the chance that a person who is a designer will match these descriptions?' measured participants' posterior probability judgements $p(H \mid D)$, representativeness' estimates rep $(D, H)$ and inverse likelihood judgements $p(D \mid H)$ for each of the five descriptions presented. The back of the questionnaire was identical except that participants were asked to make those judgements in relation to the alternative category $(\sim H=$ 'accountant'). Namely, participants were asked to answer the following questions: 'What is the chance that a person who matches this description will be an accountant?' $[p(\sim H \mid D)]$, 'On a scale from 0 to 100 , indicate the degree to which these descriptions are representative/typical of an accountant' $[\operatorname{rep}(D, \sim H)]$, and 'What is the chance that a person who is an accountant will match these descriptions?' $[p(D \mid \sim H)]$ for each of the five thumbnail descriptions presented.

\section{Results}

\section{Evaluation of normality assumption}

To warrant the use of parametric statistical tests, skewness and kurtosis statistics for $p(H \mid D), p(\sim H \mid D), p(D \mid H), p(D \mid \sim H)$, and rep $(D, H)$, rep $(D, \sim H)$ judgements were examined for $H$ and $\sim H$ and descriptions $d_{1}, d_{2}, d_{3}, d_{4}, d_{5}$. No significant departure from normality was observed $\left(z_{\text {skewness }}\right.$ and $z_{\text {kurtosis }}$ scores $\left.<3.29\right)$. Consequently, parametric statistical tests were used throughout.

\section{Order effects}

The design involved two order factors (description order and category order) and one experimental factor (judgement type). The effects of these factors on the ten dependent measures used (i.e., measures for each of five descriptions, namely, 'typical designer' $\left[\mathrm{d}_{1}\right]$, 'designer' $\left[\mathrm{d}_{2}\right]$, 'neutral' $\left[\mathrm{d}_{3}\right]$, 'accountant' $\left[\mathrm{d}_{4}\right]$, and 'typical accountant' $\left[\mathrm{d}_{5}\right]$ descriptions), and for the two target categories used (i.e., $H$ : 'designer' and $\sim \mathrm{H}$ : 'accountant', respectively) were tested by subjecting the data to a $2 \times 2 \times 3$ Multivariate Analysis of Variance (MANOVA). The multivariate tests for between-subject effects showed that neither the order of descriptions [multivariate $F(3,10)=0.32$, Wilk's $\Lambda=$ $0.48, p=.93$, partial $\eta^{2}=.52$ ] nor the order of target category [multivariate $F(3,10)=$ 1.90, Wilk's $\Lambda=0.14, p=.33, \eta^{2}=.86$ ] significantly affected judgements. Moreover, 
the interaction term for these two factors was not significant $[F(3,10)=.37$, Wilk's $\Lambda$ $=0.45, p=.90$, partial $\left.\eta^{2}=.55\right]$ so data were collapsed across order.

\section{Inverse fallacy test}

The main hypothesis of the present experiment was that inverse likelihood judgements best predicted posterior probability judgements. If confirmed, this hypothesis would thus show that individuals were committing the inverse fallacy when making posterior probability judgements. To test this hypothesis, an idiographic approach was followed. Predicted estimates [i.e., posterior probability judgements $p(H \mid D)$ ] were plotted against inverse likelihood judgements [i.e., $p(D \mid H)$ ] for each of the 16 participants who took part in the experiment. If participants were indeed making posterior probability judgements on the basis of their subjective estimate of inverse likelihood, one would expect that individual regression lines would be close to the identity line (i.e., intercept $=0$ and slope $=1$ ). Figure IV-7 presents individual scatterplots of posterior probability judgements observed for each individual participant as a function of their inverse probability judgement, for the 10 dependent measures used. Table IV-6 shows the corresponding parameter estimates for the individual regression lines. It was inferred that a participant committed the inverse fallacy if the $95 \%$ confidence interval for the intercept B coefficient included zero, the $95 \%$ confidence interval for the predictor $B$ coefficient included one, and the $R^{2}$ coefficient was greater than .80 . 


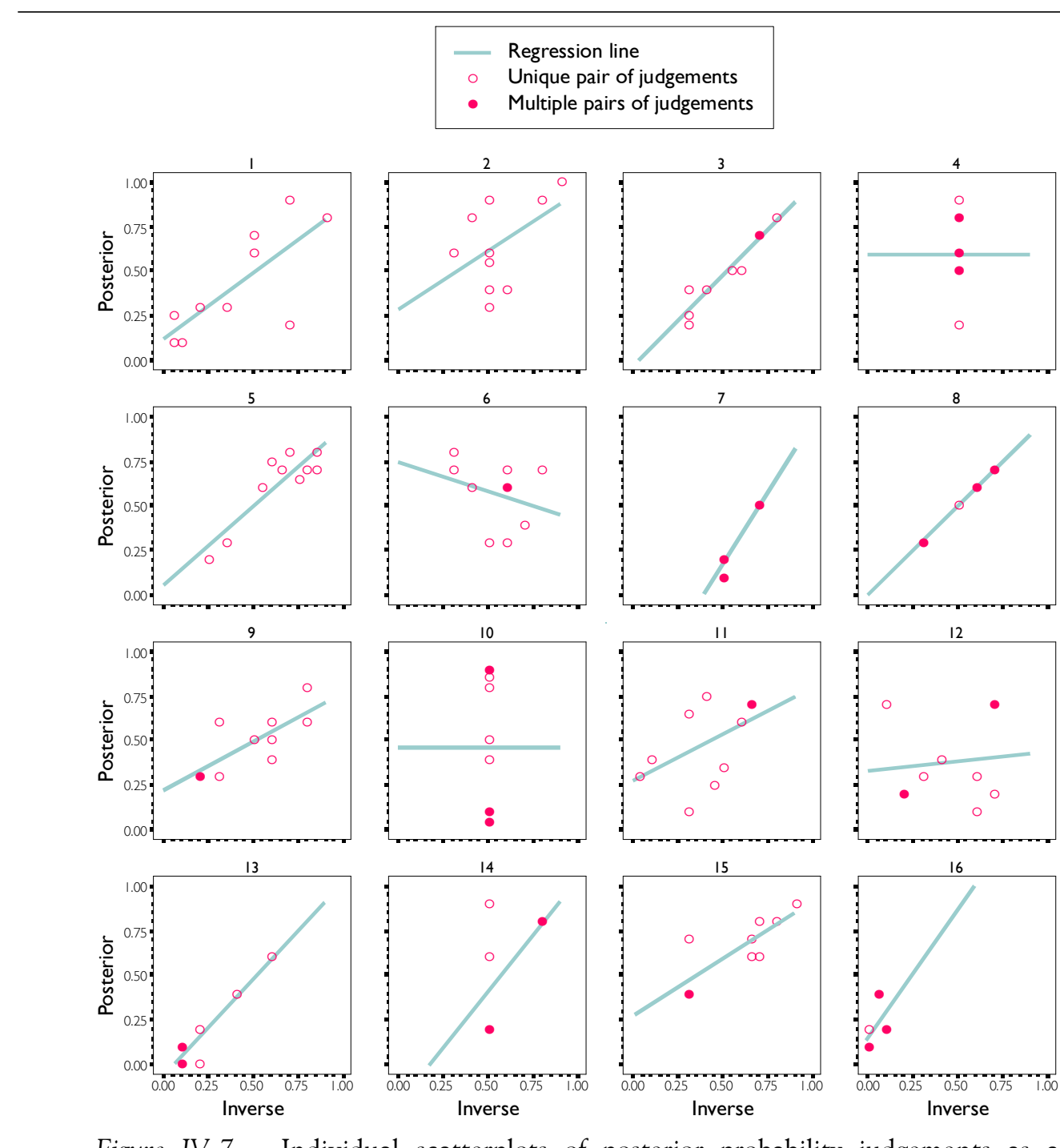

Figure IV-7 - Individual scatterplots of posterior probability judgements as a function of inverse likelihood judgements for each description and each target hypothesis. 
Table IV-6

Parameter estimates for the inverse fallacy test.

\begin{tabular}{cccccccc}
\hline & \multicolumn{3}{c}{ Intercept } & \multicolumn{5}{c}{ Predictor } \\
& \multicolumn{3}{c}{ 95\% C.I. } & \multicolumn{3}{c}{ 95\% C.I. } \\
\cline { 2 - 6 } Participant & $B$ & Lower & Upper & B & Lower & Upper & $R^{2}$ \\
\hline 1 & 0.13 & -0.14 & 0.39 & 0.74 & 0.21 & 1.27 & 0.57 \\
2 & 0.28 & -0.28 & 0.84 & 0.66 & -0.32 & 1.63 & 0.23 \\
3 & -0.03 & -0.17 & 0.11 & 1.01 & 0.77 & 1.26 & $0.92^{\dagger}$ \\
4 & 0.59 & 0.45 & 0.73 & 0.00 & 0.00 & 0.00 & 0.00 \\
5 & 0.05 & -0.20 & 0.30 & 0.89 & 0.52 & 1.27 & 0.79 \\
6 & 0.74 & 0.27 & 1.21 & -0.32 & -1.15 & 0.52 & 0.09 \\
7 & -0.64 & -0.85 & -0.42 & 1.63 & 1.23 & 2.02 & 0.92 \\
8 & 0.00 & 0.00 & 0.00 & 1.00 & 1.00 & 1.00 & $1.00^{\dagger}$ \\
9 & 0.22 & 0.01 & 0.43 & 0.55 & 0.16 & 0.94 & 0.57 \\
10 & 0.47 & 0.20 & 0.73 & 0.00 & 0.00 & 0.00 & 0.00 \\
11 & 0.27 & -0.06 & 0.60 & 0.52 & -0.22 & 1.26 & 0.25 \\
12 & 0.34 & -0.07 & 0.74 & 0.10 & -0.70 & 0.90 & 0.01 \\
13 & -0.06 & -0.14 & 0.03 & 1.08 & 0.75 & 1.41 & $0.88^{\dagger}$ \\
14 & -0.21 & -0.95 & 0.52 & 1.27 & 0.17 & 2.36 & 0.47 \\
15 & 0.27 & 0.05 & 0.49 & 0.64 & 0.27 & 1.00 & 0.67 \\
16 & 0.15 & 0.05 & 0.25 & 1.44 & -0.56 & 3.43 & 0.26 \\
\hline${ }^{\dagger} B_{\text {Intercept }}=0, B_{\text {Inverse }}=1$, and $R^{2} \geq .80$ & & & & \\
\hline & & & & & & &
\end{tabular}

According to this classification scheme, only three out of the sixteen participants (namely participants \#3, \#8 and \#13) could be classified as following an inverse judgement method when evaluating the posterior probability judgement. Moreover, the extent to which inverse likelihood judgements accounted for the posterior probability judgements observed varied substantially among participants as $R^{2}$ coefficients ranged from 0 to 1 .

To test whether participants' individual regression lines were homogeneous, the posterior probability judgements were subjected to a 16-within (Participant) one-way Analysis of Covariance (ANCOVA) with one covariate, Inverse likelihood judgement. The effect of the Inverse likelihood covariate was significant $[F(1,130)=33.18, p<$ $.001, \eta^{2}=.20$, indicating that posterior probability judgements could be reliably predicted from the knowledge of inverse judgements. The interaction term Participant $\times$ Inverse likelihood was also significant at the .10 level $[F(13,130)=1.62$, MSE $=$ 0.03, $\left.p=.09, \eta^{2}=.14\right]$. This result shows that the regression coefficients for the 
Inverse likelihood covariate could not be assumed to be homogeneous across participants. In other words, contrary to what was originally hypothesised, the extent to which the inverse likelihood judgements predicted posterior probability judgements significantly varied across participants, thereby indicating that the inverse fallacy did not account well, overall, for individuals' posterior probability judgements.

\section{Representativeness test}

The main hypothesis tested above was derived from suggestions within the literature (e.g., see Gigerenzer \& Murray, 1987) that the so-called representativeness heuristic was in fact an instance of the inverse fallacy. Although the results just presented show that one cannot conclude that people generally confused posterior probability judgements and inverse likelihood judgements in Social-judgement tasks, it is possible that the representativeness heuristic is in fact a better descriptor of posterior probability judgements. To test this hypothesis, posterior probability judgements for each of the five descriptions used and for each of the 16 participants who took part in the study were plotted against their corresponding representativeness estimates (cf. Figure IV-8). Table IV-7 shows the corresponding parameter estimates for each individual regression line. The same criteria for classification were used to identify judgement methods based on representativeness. Namely, the use of a representativeness heuristic was inferred when the $95 \%$ confidence interval for the intercept B coefficient included zero, the $95 \%$ confidence interval for the predictor $B$ coefficient included one, and the $R^{2}$ coefficient was greater than .80 . 

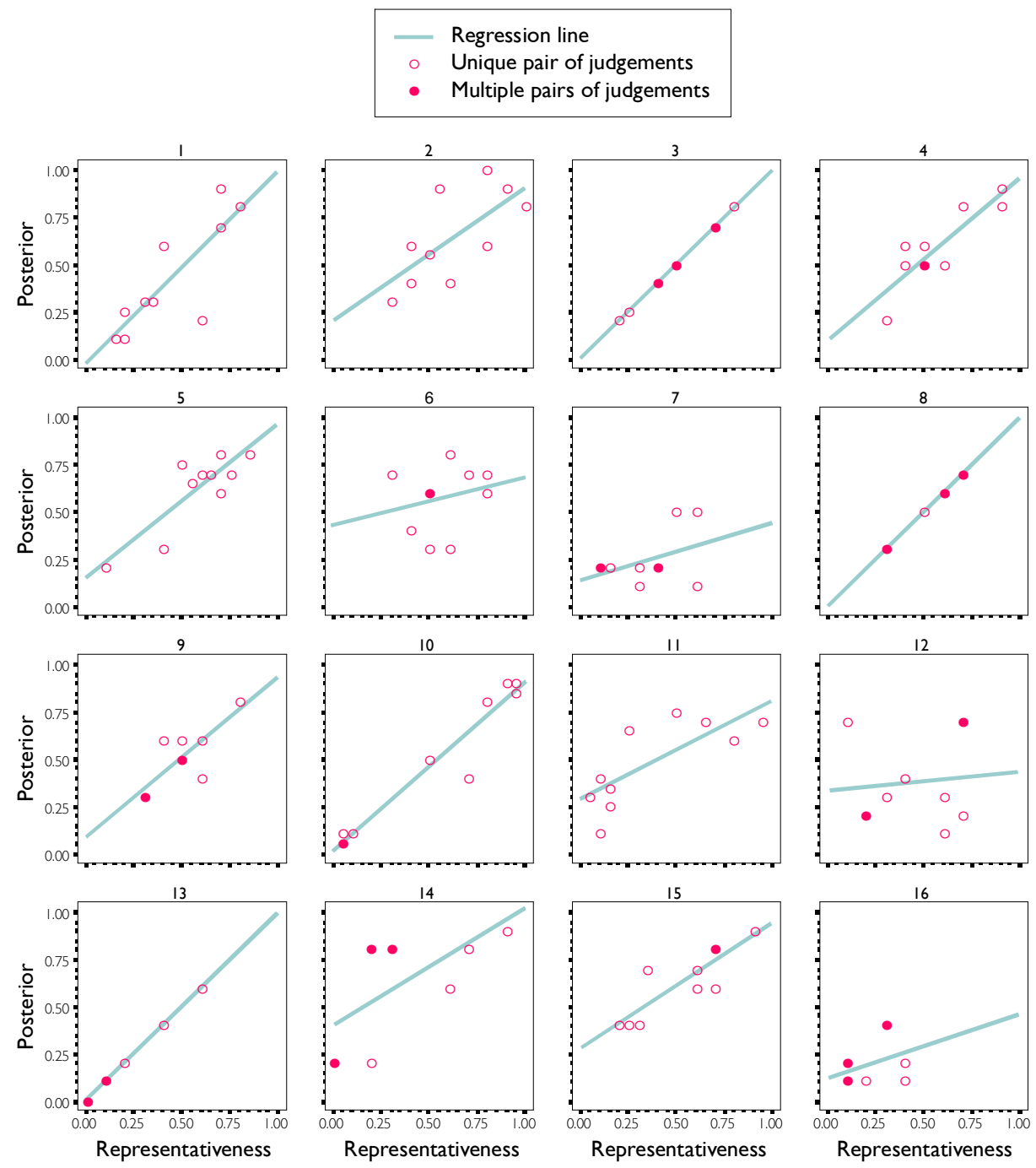

Figure IV-8 - Individual scatterplots of posterior probability judgements as a function of representativeness judgements for each description and each target hypothesis. 
Table IV-7

Parameter estimates for the representativeness heuristic test.

\begin{tabular}{|c|c|c|c|c|c|c|c|}
\hline \multirow[b]{3}{*}{ Participant } & \multicolumn{3}{|c|}{ Intercept } & \multicolumn{3}{|c|}{$\begin{array}{c}\text { Predictor } \\
\text { (Representativeness estimate) }\end{array}$} & \multirow[b]{3}{*}{$R^{2}$} \\
\hline & \multicolumn{3}{|c|}{ 95\% C.I. } & \multicolumn{3}{|c|}{ 95\% C.I. } & \\
\hline & B & Lower & Upper & $B$ & Lower & Upper & \\
\hline 1 & -.02 & -.31 & .26 & 1.02 & .45 & 1.59 & .68 \\
\hline 2 & .20 & -.20 & .60 & .71 & .10 & 1.31 & .48 \\
\hline 3 & .00 & .00 & .00 & 1.00 & 1.00 & 1.00 & $1.00^{\circ}$ \\
\hline 4 & .10 & -.14 & .34 & .86 & .47 & 1.25 & .76 \\
\hline 5 & .14 & -.11 & .39 & .83 & .42 & 1.24 & .73 \\
\hline 6 & .43 & -.08 & .93 & .25 & -.60 & 1.11 & .06 \\
\hline 7 & .13 & -.09 & .35 & .32 & -.24 & .88 & .18 \\
\hline 8 & .00 & .00 & .00 & 1.00 & 1.00 & 1.00 & 1.00 \\
\hline 9 & .09 & -.16 & .33 & .84 & .36 & 1.32 & .67 \\
\hline 10 & .01 & -.11 & .13 & .90 & .72 & 1.082 & .94 \\
\hline 11 & .28 & .11 & .46 & .53 & .17 & .89 & .59 \\
\hline 12 & .34 & -.07 & .74 & .10 & -.71 & .90 & .01 \\
\hline 13 & .00 & .00 & .00 & 1.00 & 1.00 & 1.00 & 1.00 \\
\hline 14 & .40 & .13 & .67 & .62 & .00 & 1.24 & .40 \\
\hline 15 & .28 & .09 & .46 & .66 & .34 & .98 & .74 \\
\hline 16 & .12 & -.05 & .29 & .34 & -.36 & 1.05 & .14 \\
\hline
\end{tabular}

These results show that only a handful of individuals (i.e., participants \#3, \#8, \#10, and \#13) systematically assimilated posterior probability and representativeness judgements. And, as for the inverse fallacy test, the proportion of variation explained by a model based on representativeness estimates ranged from .01 to 1 , showing that whereas some participants' posterior probability judgements were perfectly predicted by their representativeness estimates, others' posterior probability judgements were absolutely unrelated to those estimates.

To test whether participants' individual regression lines were homogeneous, the posterior probability judgements $p(H \mid D)$ were subjected to a 16-within (Participant) one-way Analysis of Covariance (ANCOVA) with the representativeness judgements $\operatorname{rep}(D, H)$ as covariate (Representativeness). As for the Inverse likelihood covariate, the effect of the Representaveness covariate reached statistical significance $[F(1,128)=$ 135.66, $p<.0001$ ], showing that posterior probability judgements could indeed be reliably predicted from representativeness estimates. On the whole, the size of this effect 
was substantial [partial $\eta^{2}=.52$ ]. Yet, as with the inverse likelihood covariate, the interaction term Participant $\times$ Representativeness was statistically significant $[F(15$, $128)=1.80, \mathrm{MSE}=0.02, p<.05$, partial $\left.\eta^{2}=.17\right]$. This result shows that the extent to which the representativeness judgements predicted observed posterior probability judgements significantly varied among participants.

So, although posterior probability judgements can reliably be predicted from the knowledge of inverse likelihood judgements or from the knowledge of representativeness judgements, these results show that few participants systematically relied on either the inverse fallacy or the representativeness heuristic to form their posterior probability judgements. In fact, the posterior judgements of only four out of 16 participants could be modelled by either of these accounts (i.e., participants \#3, \#8, \#10, and \#13), or even both (i.e., posterior judgements of participants \#3, \#8, and \#13 were equally accounted for by their inverse likelihood judgements and their representativeness judgements). Consequently, the processes underlying the judgements of the remaining 12 participants remain unaccounted for. A question arising, therefore, is whether these latter participants' posterior judgements can be described by a Bayesian account or else by a "base-rate-neglect-but-otherwise-Bayesian" (BRNBOB) account. These possibilities are investigated in the following subsection.

\section{BRNBOB and Bayesian tests}

The collection of posterior probability estimates for each of two complementary categories (namely, 'designer,' and 'accountant') and the presence of base-rate information in the tasks used allowed for the computation of individual Bayesian values. For instance, Bayesian values for the designer target category or $p(H \mid D)$ values could be inferred for each participant on each of the descriptions used by calculating $.70 \times$ $p(D \mid H) /[.70 \times p(D \mid H)+.30 \times p(D \mid \sim H)]$, where $p(D \mid H)$ and $p(D \mid \sim H)$ represent the inverse likelihood judgements for the category 'designer' and the category 'accountant,' respectively. Similarly, in order to test the hypothesis that participants only neglected base-rate information (i.e., assuming that their estimates were based on the correct ratio of likelihoods, but were not weighted by base-rates), BRNBOB (Base-rateneglect-but-otherwise-Bayesian) values were inferred for each participant on each of the descriptions used by calculating, e.,g., for the 'designer' target category, $p(D \mid H) /$ $[p(D \mid H)+p(D \mid \sim H)]$. To warrant the use of parametric statistical tests, skewness and kurtosis statistics for BRNBOB and Bayesian values were computed. No significant 
departure from normality was observed $\left(z_{\text {skewness }}\right.$ and $z_{\text {kurtosis }}$ scores $\left.<3.29\right)$. Consequently, parametric statistical tests were used throughout.

To test whether posterior probability judgements were based on a BRNBOB strategy, posterior probability judgements were plotted against their corresponding BRNBOB values for each participant (cf. Figure IV-9). Table IV-8 shows the corresponding parameters estimates for each individual regression line.

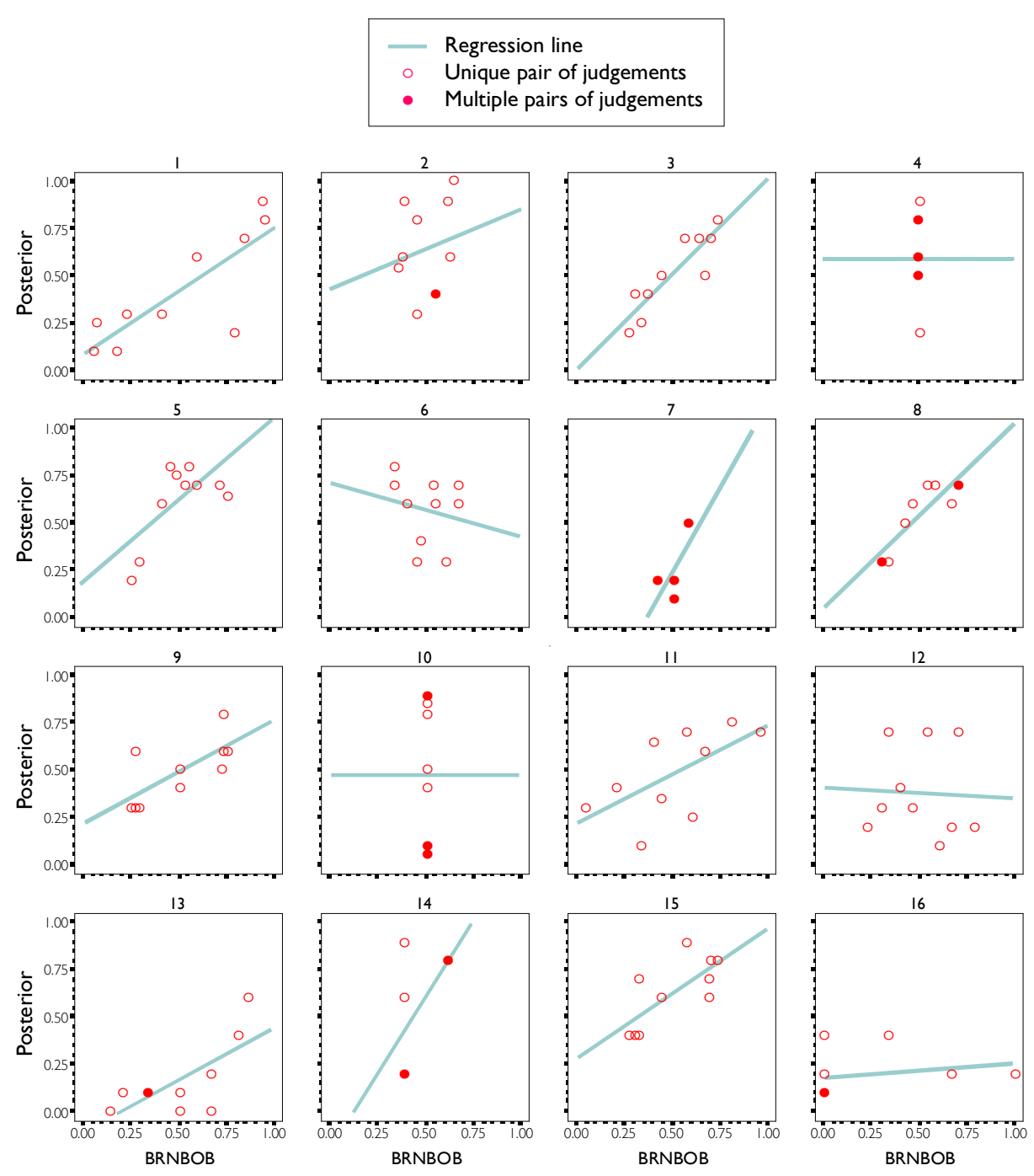

Figure IV-9 - Individual scatterplots of posterior probability judgements as a function of $\mathrm{BRNBOB}$ values for each of description and each target hypothesis used.

Note. BRNBOB stands for 'Base-Rate-Neglect-But-Otherwise-Bayesian'. 
Table IV-8

Parameter estimates for the BRNBOB test.

\begin{tabular}{|c|c|c|c|c|c|c|c|}
\hline \multirow[b]{3}{*}{ Participant } & \multicolumn{3}{|c|}{ Intercept } & \multicolumn{3}{|c|}{$\begin{array}{c}\text { Predictor } \\
\text { (BRNBOB value) }\end{array}$} & \multirow[b]{3}{*}{$R^{2}$} \\
\hline & \multicolumn{3}{|c|}{ 95\% C.I. } & \multicolumn{3}{|c|}{$95 \%$ C.I. } & \\
\hline & B & Lower & Upper & $B$ & Lower & Upper & \\
\hline 1 & .09 & -.15 & .32 & .68 & .30 & 1.06 & .68 \\
\hline 2 & .43 & -.47 & 1.33 & .43 & -1.34 & 2.20 & .04 \\
\hline 3 & .00 & -.23 & .23 & 1.03 & .59 & 1.47 & .79 \\
\hline 4 & .59 & .45 & .74 & .00 & .00 & .00 & .00 \\
\hline 5 & .19 & -.21 & .58 & .87 & .11 & 1.63 & .47 \\
\hline 6 & .71 & .13 & 1.30 & -.29 & -1.43 & .85 & .04 \\
\hline 7 & -.66 & -1.41 & .09 & 1.8 & .30 & 3.30 & .49 \\
\hline 8 & .04 & -.16 & .25 & .99 & .594 & 1.39 & .81 \\
\hline 9 & .22 & -.02 & .45 & .55 & .11 & .99 & .51 \\
\hline 10 & .47 & .20 & .73 & .00 & .00 & .00 & .00 \\
\hline 11 & .22 & -.80 & .52 & .52 & -.01 & 1.05 & .40 \\
\hline 12 & .41 & -.14 & .95 & -.06 & -1.08 & .97 & .00 \\
\hline 13 & -.12 & -.37 & .14 & .56 & .09 & 1.02 & .49 \\
\hline 14 & -.21 & -.95 & .52 & 1.65 & .22 & 3.07 & .47 \\
\hline 15 & .28 & .00 & .57 & .69 & .15 & 1.23 & .52 \\
\hline 16 & .18 & .07 & .28 & .08 & -.19 & .34 & .05 \\
\hline
\end{tabular}

According to the three criteria previously used (namely, the 95\% confidence interval for the intercept B coefficient included zero, the $95 \%$ confidence interval for the predictor $B$ coefficient included one, and the $R^{2}$ coefficient was greater than .80 ), only one participant (participant \#8) could be assumed to have used the correct ratio of likelihood but neglected base-rate information. This participant's judgements, however, were also accounted by the inverse fallacy or the representativeness heuristic. To test whether posterior probability judgements could be reliably predicted from BRNBOB values, they were subjected to a One-Way ANCOVA with Participant as a factor and $\mathrm{BRNBOB}$ as a covariate. Once more, the effect of the covariate was significant $[F(1$, 130) $=28.19$, MSE $=0.04, p<.0001$, partial $\left.\eta^{2}=.18\right]$, showing that posterior probability judgements could indeed be reliably predicted from BRNBOB values.

Tests for homogeneity of regression, however, revealed that the interaction Participant $\times$ BRNBOB was significant at the $.10 \alpha$-level, $[F(13,130)=1.72$, MSE $=$ $0.04, p=.06$, partial $\left.\eta^{2}=.15\right]$, indicating that the regression coefficients of the 
individual regression lines could not be assimilated, thus showing that the BRNBOB strategy did not provide a general computational account for posterior probability judgements observed.

Figure IV-10 shows the same plot but with posterior probability judgements plotted against corresponding Bayesian values. Table IV-9 shows the corresponding parameter estimates for each individual regression line.

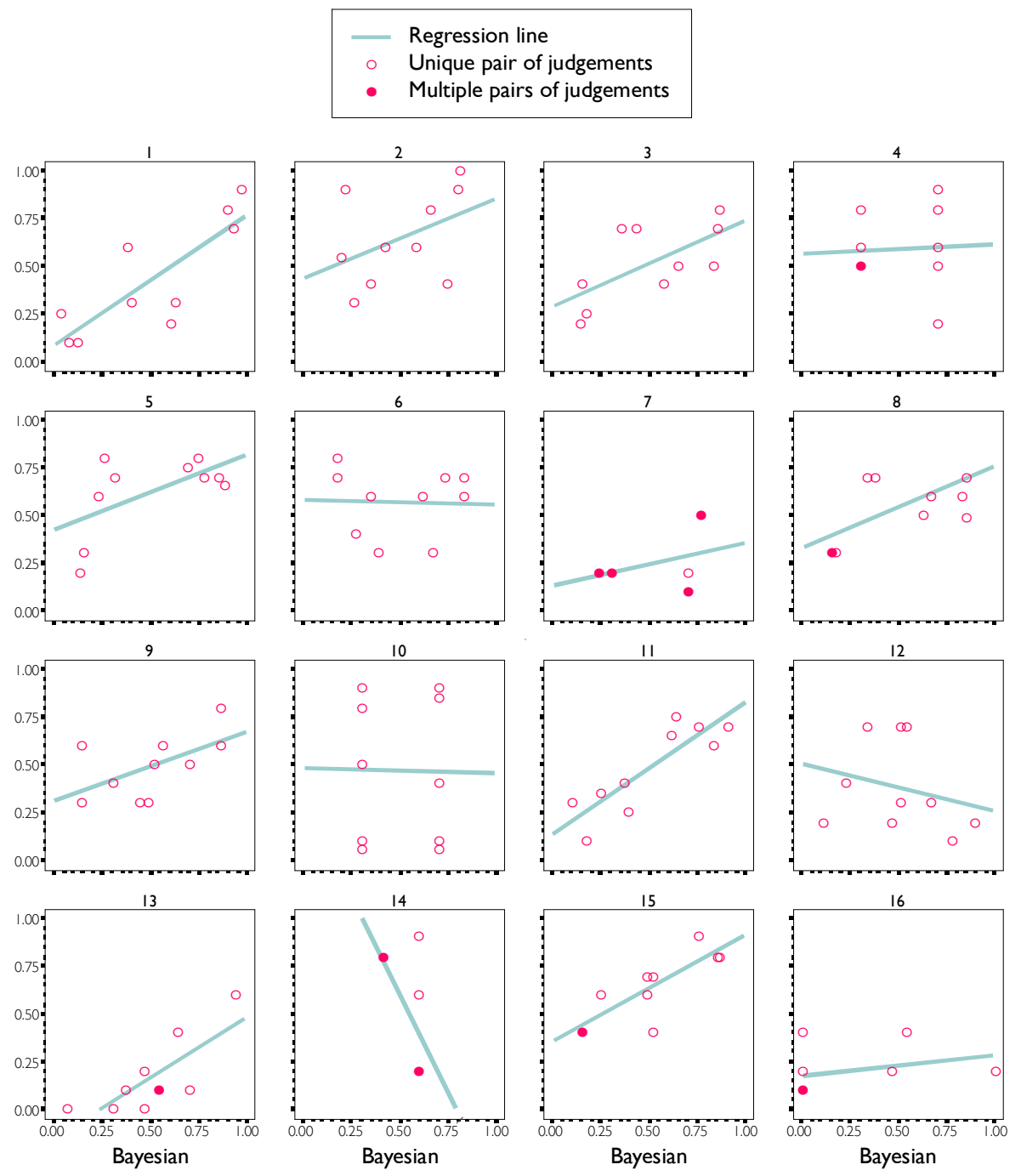

Figure IV-10 - Individual scatterplots of posterior probability judgements as a function of Bayesian values for each description and each target hypothesis. 
Table IV-9

Parameter estimates for the Bayesian test.

\begin{tabular}{|c|c|c|c|c|c|c|c|}
\hline \multirow[b]{3}{*}{ Participant } & \multicolumn{3}{|c|}{ Intercept } & \multicolumn{3}{|c|}{$\begin{array}{c}\text { Predictor } \\
\text { (Bayesian value) }\end{array}$} & \multirow[b]{3}{*}{$R^{2}$} \\
\hline & \multicolumn{3}{|c|}{ 95\% C.I. } & \multicolumn{3}{|c|}{ 95\% C.I. } & \\
\hline & B & Lower & Upper & B & Lower & Upper & \\
\hline 1 & .08 & -.15 & .32 & .68 & .29 & 1.07 & .67 \\
\hline 2 & .43 & .03 & .84 & .42 & -.32 & 1.17 & .18 \\
\hline 3 & .29 & .04 & .54 & .46 & .02 & .90 & .42 \\
\hline 4 & .57 & .14 & .99 & .05 & -.73 & .83 & .00 \\
\hline 5 & .42 & .17 & .67 & .40 & -.03 & .84 & .37 \\
\hline 6 & .58 & .27 & .89 & -.02 & -.58 & .54 & .00 \\
\hline 7 & .13 & -.12 & .37 & .23 & -.22 & .68 & .15 \\
\hline 8 & .33 & .13 & .53 & .43 & .08 & .78 & .50 \\
\hline 9 & .31 & .07 & .55 & .37 & -.06 & .79 & .33 \\
\hline 10 & .48 & -.30 & 1.26 & -.030 & -1.47 & 1.42 & .00 \\
\hline 11 & .13 & -.06 & .32 & .70 & .37 & 1.03 & .75 \\
\hline 12 & .51 & .08 & .93 &. .25 & -1.02 & .52 & .07 \\
\hline 13 & -.16 & -.40 & .07 & .64 & .21 & 1.07 & .60 \\
\hline 14 & 1.63 & .73 & 2.53 & -2.04 & -3.81 & -.27 & .47 \\
\hline 15 & .35 & .16 & .53 & .57 & .24 & .89 & .67 \\
\hline 16 & .17 & .07 & .27 & .12 & -.15 & .38 & .12 \\
\hline
\end{tabular}

This time, none of the individual regression lines was confounded with the identity line, thereby indicating that none of the participants exhibited posterior probability judgements that could result from Bayesian computations using the base-rate information presented in the problem. A One-Way ANCOVA with Participant as a factor and Bayesian values as covariate (Bayesian) revealed that although the effect of the Bayesian covariate was statistically significant $[F(1,128)=5.41, \mathrm{MSE}=0.04, p<$ $.05]$, it accounted for very little variance $\left(\eta^{2}=.04\right)$. And, once more, the interaction term Participant $\times$ Bayesian was statistically significant, indicating that the slopes of individual regression lines were not homogeneous and varied as a function of participants $\left[F(15,128)=2.15\right.$, MSE $=0.04, p<.05$, partial $\left.\eta^{2}=.20\right]$.

\section{Individual profiles}

In sum, individuals' posterior probability judgements could not, on the whole, be assumed to be based on the inverse fallacy, the representativeness heuristic, a Bayesian strategy, or even a Bayesian strategy where only base-rate information was ignored 
(BRNBOB). Different individuals appeared to be using different strategies to make their judgements. Contrary to what was originally hypothesized, participants' posterior probability judgements were never solely linearly related to their inverse likelihood judgements. Contrary to what was suggested in the literature (e.g., see Gigerenzer \& Murray, 1987; Pollard \& Evans, 1983), this shows that, when compared to the representativeness heuristic, the inverse fallacy is not a better descriptor of the computational processes underlying judgements. Still, there was no overwhelming indication that individuals were solely relying on the representativeness heuristic. Interestingly, only one out of the 16 participants exhibited posterior probability judgements that were exclusively linearly related to their representativeness judgements (participant \#10). In other cases, posterior probability judgements were related with either both inverse likelihood and representativeness judgements (e.g., participants \#3 and \#13) or even inverse likelihood, representativeness and BRNBOB judgements (e.g., see participant \#8).

To study the different patterns exhibited by each individuals, the "comparability" and "incomparability" relations of 'strategy profiles' were investigated using the underlying principles of partial-order scalogram analysis (POSA) (Shye, Elizur, \& Hoffman, 1994). Specifically, to 'measure' the strategies used by different individuals; that is, to scale those strategies "so as to suggest comparison of individuals on an ordinal basis, in a 'ladder-like arrangement'; [Oxford Dictionary]"; Shye et al., 1994, p. 136), the classification between posterior probability judgements and each of the four predictors (inverse likelihood, representativeness, BRNBOB, and Bayesian) were transformed into binary variables (with $1=$ positive match and $0=$ mismatch). The resulting strategy profiles for each individual are summarised in Table IV-10. 
Table IV-10

Strategy profiles of the 16 participants.

\begin{tabular}{ccccc}
\hline & \multicolumn{5}{c}{ Components } \\
\cline { 2 - 5 } Participants & IF & RH & BN & B \\
\hline$\# 1$ & 0 & 0 & 0 & 0 \\
$\# 2$ & 0 & 0 & 0 & 0 \\
$\# 3$ & 1 & 1 & 0 & 0 \\
$\# 4$ & 0 & 0 & 0 & 0 \\
$\# 5$ & 0 & 0 & 0 & 0 \\
$\# 6$ & 0 & 0 & 0 & 0 \\
$\# 7$ & 0 & 0 & 0 & 0 \\
$\# 8$ & 1 & 1 & 1 & 0 \\
$\# 9$ & 0 & 0 & 0 & 0 \\
$\# 10$ & 0 & 1 & 0 & 0 \\
$\# 11$ & 0 & 0 & 0 & 0 \\
$\# 12$ & 0 & 0 & 0 & 0 \\
$\# 13$ & 1 & 1 & 0 & 0 \\
$\# 14$ & 0 & 0 & 0 & 0 \\
$\# 15$ & 0 & 0 & 0 & 0 \\
$\# 16$ & 0 & 0 & 0 & 0 \\
\hline
\end{tabular}

Note. IF, RH, BN, and BV correspond to the strategies tested, namely Inverse Fallacy, Representativeness Heuristic, Base-rate-Neglect-but-otherwise-Bayesian, and Bayesian strategies.

The value 1 indicates that posterior probability judgements could be predicted by the corresponding strategy. 0 indicates that posterior probability judgements could not be reliably predicted by the corresponding strategy.

Comparability and incomparability of the strategy profiles were determined according to the following principles,

Given two different profiles, one profile is said to be greater than another only if it is greater or equal to the other in each and every score; and if it is strictly greater than the other in at least one score. Otherwise the two profiles are incomparable. (Shye et al., 1994, p. 137)

Figure IV-11 shows a graphical representation of these relations, called 'Hasse diagram' (Shye et al., 1994). 
(\#I, 2, 4, 5, 6, 7, 9, II, I2, I4, I5, I6)

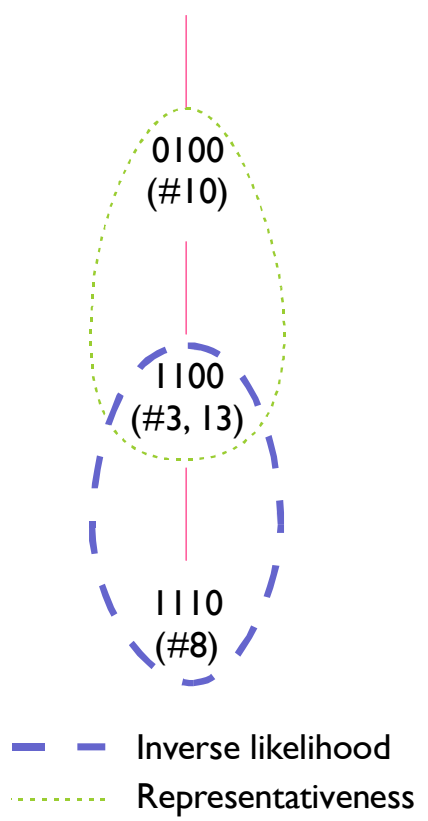

Figure IV-11 - Hasse diagram of the 16 mutually comparable strategy profiles.

Downward directed lines represent comparability relations between profiles. The lower end of a line always points to a greater profile. The resulting Hasse diagram shows that the vast majority of participants' posterior probability judgements were not linearly related to any of the four components (profile 0000). It also appears that no participants made posterior judgements that were solely linearly related to their inverse likelihood judgements (as indicated by the absence of the profile 1000). In fact, when posterior judgements were related to inverse likelihood judgements, they were also related to representativeness judgements (profile 1100) or both representativeness and base-rateneglect-but-otherwise-Bayesian (profile 1110), thus making it impossible to reliably infer the processes that may have underlain the observed judgement outcomes. Finally, this graph also shows that only 3 out of 16 participants exhibited posterior judgements that were related to their inverse likelihood judgements (profiles beginning with 1), but all these participants also exhibited judgements that were linearly related to their judgements of representativeness. It was only possible to unambiguously determine the strategy or computational process underlying the judgements of participant \#10 whose posterior probability judgements could only be reliably predicted by its representativeness judgements. This clearly illustrates the limits of outcome-based measures to fulfil the initial aim of a process-focused approach, namely to provide a 
systematic description of the judgement processes underlying the outcomes observed. Although it is clear that most individuals' posterior probability judgements were not in line with Bayesian prescriptions (as no participant exhibited judgements that were indeed linearly related to Bayesian prescriptions), the processes by which they arrived at their judgement could not generally be unambiguously inferred from the present data. This also demonstrates that on the whole very few individuals may indeed rely on the representativeness heuristic to make posterior probability judgements in Socialjudgement tasks.

\section{Deviations from the additivity principle}

The last hypotheses of this experiment concerned the additivity issue. First, it was hypothesized that most participants would not respect the additivity principle. To test this hypothesis, Kahneman and Tversky (1973) computed the median probabilities assigned to each of the two alternative categories for each of the descriptions presented. The authors assumed that their participants had obeyed the additivity principle since the sum of those median estimates "added to about 100\% for each description" (p. 241). The same analysis is summarised in Table IV-11. In this table, the median estimates for $p(H \mid D)$ judgements (category 'designer') and for $p(\sim H \mid D)$ judgements (category 'accountant') are included, as well as their sum.

Table IV-11

Median $p(H \mid D)$ and $p(\sim H \mid D)$ posterior probabilities judgements, for each description

\begin{tabular}{cccc}
\hline & \multicolumn{2}{c}{ Median } & \\
\cline { 2 - 3 } Description & $p(H \mid D)$ & $p(\sim H \mid D)$ & Sum \\
\hline $\mathrm{d}_{1}$ & 0.60 & 0.28 & 0.88 \\
$\mathrm{~d}_{2}$ & 0.70 & 0.38 & 1.08 \\
$\mathrm{~d}_{3}$ & 0.60 & 0.35 & 0.95 \\
$\mathrm{~d}_{4}$ & 0.35 & 0.65 & 1.00 \\
$\mathrm{~d}_{5}$ & 0.30 & 0.70 & 1.00 \\
\hline
\end{tabular}

Note. $H$ and $\sim H$ refer to the categories 'designer' and 'accountant', respectively.

Descriptions $\mathrm{d}_{1}, \mathrm{~d}_{2}, \mathrm{~d}_{3}, \mathrm{~d}_{4}$, and $\mathrm{d}_{5}$ refer to the typical designer, designer, neutral, accountant and typical accountant descriptions respectively, as presented in Table IV-2.

A similar conclusion to the conclusion observed by Kahneman and Tversky (1973) in view of their own data could be drawn here. Namely, additivity could be assumed 
since the sum of median estimates is generally close to 1 . This approach, however, does not inform the extent to which individual participants were following the additivity principle. To test this latter proposition, the posterior probability judgements for the 'designer' category [i.e., $p(H \mid D)$ for $i=1$ to 5] were plotted against the posterior probability judgements for the alternative 'accountant' category [i.e., $p(\sim H \mid D)$ for $i=1$ to 5], for each participant. Figure IV-12 show those plots as well as their corresponding regression lines.

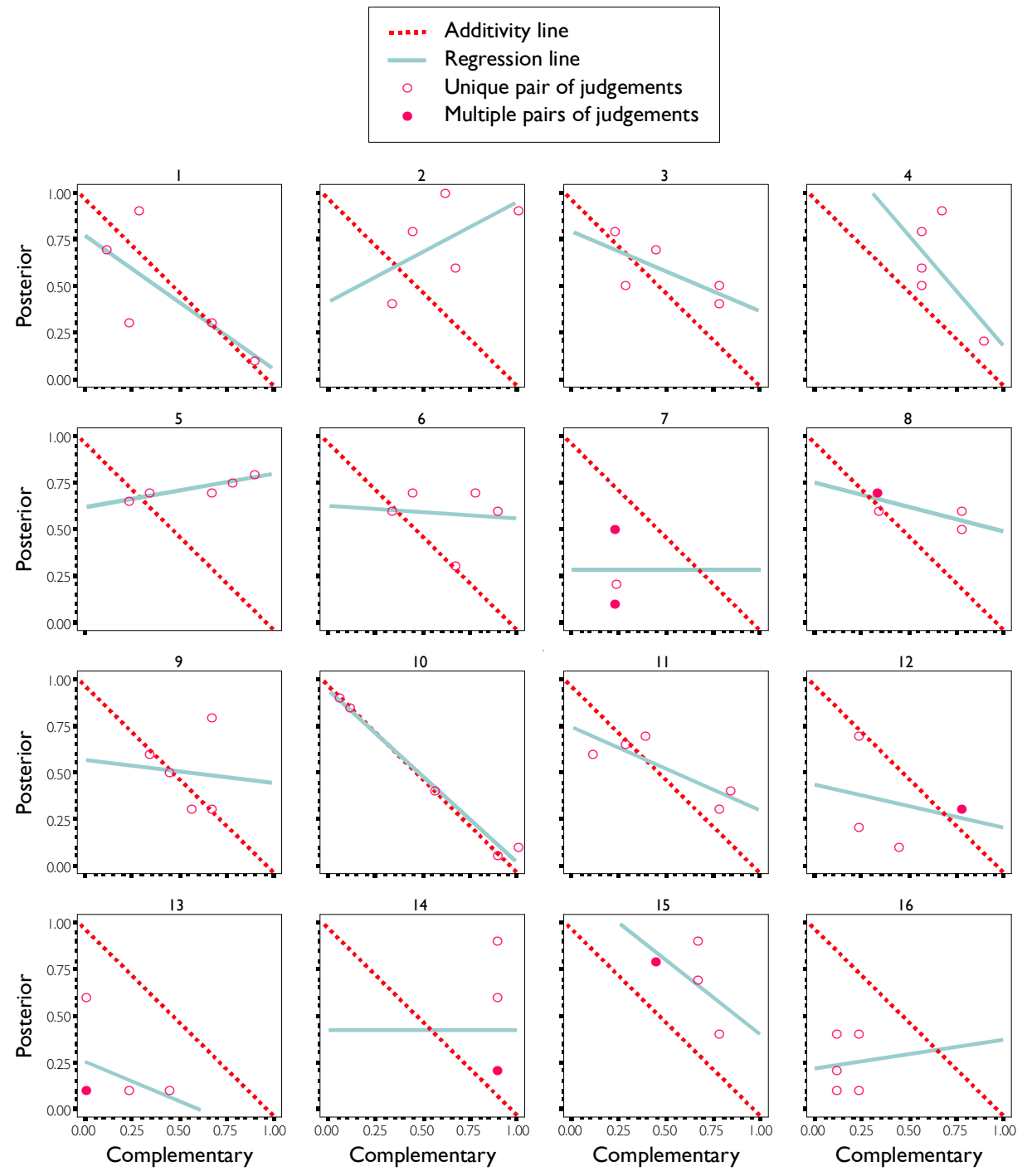

Figure IV-12 - Individual scatterplot of the posterior probability judgements for the 'designer' category as a function of the posterior probability judgements for the 'accountant' category.

Note. This illustrates the additivity test. If participants' complementary judgements were additive, one would expect these judgements to be consistent with the regression line $Y=1-X$, as indicated by the dashed line. Subadditivity occurs when paired judgements fall above the additivity line, and superadditivity, when paired judgements fall below the additivity line. 
Table IV-12 shows the parameter estimates for each individual regression line. Estimates were classified as complementary when the following three criteria were met: the $B$ coefficient for the intercept was not statistically different from 1 , the $B$ coefficient for the complementary judgement was not statistically different from -1 and when the $R^{2}$ coefficient was at least .80 .

Table IV-12

Parameter estimates for the additivity test.

\begin{tabular}{|c|c|c|c|c|c|c|c|}
\hline \multirow[b]{3}{*}{ Participant } & \multicolumn{3}{|c|}{ Intercept } & \multicolumn{3}{|c|}{$\begin{array}{l}\text { Predictor } \\
p(\sim H \mid D)\end{array}$} & \multirow[b]{3}{*}{$R^{2}$} \\
\hline & \multicolumn{3}{|c|}{ 95\% C.I. } & \multicolumn{3}{|c|}{ 95\% C.I. } & \\
\hline & B & Lower & Upper & $B$ & Lower & Upper & \\
\hline 1 & .77 & .11 & 1.44 & -.80 & -2.21 & .61 & .52 \\
\hline 2 & .41 & -.52 & 1.35 & .60 & -1.00 & 2.19 & .32 \\
\hline 3 & .80 & .35 & 1.25 & -.48 & -1.38 & .42 & .49 \\
\hline 4 & 1.37 & -.41 & 3.14 & -1.32 & -4.32 & 1.67 & .40 \\
\hline 5 & .62 & .52 & .72 & .20 & .02 & .38 & .81 \\
\hline 6 & .63 & -.23 & 1.48 & -.08 & -1.53 & 1.37 & .01 \\
\hline 7 & .28 & .03 & .53 & .00 & .00 & .00 & .00 \\
\hline 8 & .75 & .53 & .98 & -.29 & -.75 & .16 & .58 \\
\hline 9 & .57 & -.90 & 2.04 & -.15 & -3.12 & 2.83 & .01 \\
\hline 10 & .94 & .79 & 1.10 & -1.02 & -1.29 & -.76 & $.98^{\dagger}$ \\
\hline 11 & .75 & .44 & 1.06 & -.50 & -1.12 & .12 & .69 \\
\hline 12 & .43 & -.36 & 1.22 & -.25 & -1.86 & 1.35 & .08 \\
\hline 13 & .26 & -.17 & .68 & -.47 & -2.60 & 1.66 & .14 \\
\hline 14 & .42 & .02 & .82 & .00 & .00 & .00 & .00 \\
\hline 15 & 1.20 & .06 & 2.34 & -.89 & -2.96 & 1.18 & .38 \\
\hline 16 & .22 & -.54 & .97 & .17 & -4.91 & 5.25 & .00 \\
\hline
\end{tabular}

These results suggest that all but one participant (Participant \#10) violated the additivity principle. This conclusion is remarkably at odds with the conclusion based on the median estimates.

To test whether posterior probability judgements for one target category could be reliably predicted from the knowledge of the probability estimates for the alternative category, $p(H \mid D)$ judgements were subjected to a One-way Analysis of Covariance (ANCOVA) with Participant as a factor and $p(\sim H \mid D)$ judgements as a covariate (Alternative). The interaction term Participant $\times$ Alternative was not significant $[F(13$, 
50) $=1.30, \mathrm{MSE}=0.04, p=.25$, partial $\left.\eta^{2}=.25\right]$, indicating that the regression coefficients of the individual regression lines were not significantly different from each other. However, parameter estimates of a model including only the complementary covariate reveals that overall, posterior probability judgements for the 'designer' category were not normatively related to posterior probability judgements for the 'accountant' category, although the two categories were defined as complementary and mutually exhaustive in the text. Indeed, if judgements were complementary, one would expect the relation $p(H \mid D)=1-p(\sim H \mid D)$ to hold; that is, that the intercept $B$ value would be expected not be statistically different from 1 and that the predictor $B$ value would be expected not be statistically different from -1 . The overall regression line, however, was far from what would have been expected normatively $\left(B_{\text {Intercept }}=.49, \mathrm{CI}=[.37 ; .61]\right.$ and $\left.B_{\text {Complementary }}=.02, \mathrm{CI}=[.21 ; .25], R^{2}=.00\right)$. Figure IV-13 further illustrates this result by presenting the overall plot of $p(H \mid D)$ judgements against their complementary $p(\sim H \mid D)$ judgements. Altogether, these results demonstrate that posterior probability judgements observed generally violated the additivity principle.

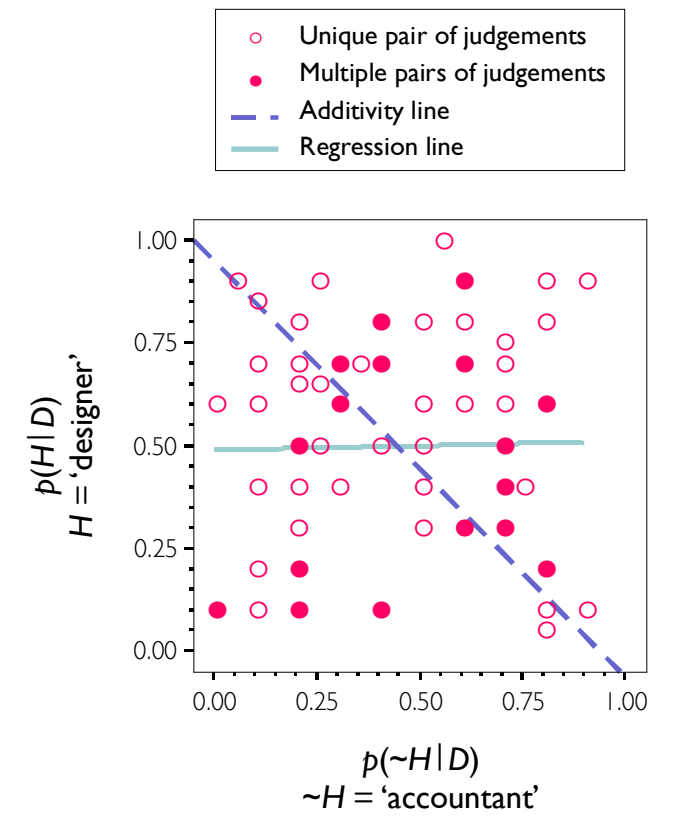

Figure IV-13 - Overall scatterplot of the posterior probability judgements for the 'designer' category as a function of the posterior probability judgements for the 'accountant' category.

In sum, as predicted, individual participants generally did not respect that additivity principle. Assuming that participants committed the inverse fallacy when making posterior probability judgements, the last hypothesis advanced for this study was that 
deviations from additivity could be predicted from the knowledge of inverse likelihood judgements for each of the two complementary categories investigated. To test whether the sum of $p\left(H \mid d_{i}\right)$ and $p\left(\sim H \mid d_{i}\right)$ posterior probability judgements could be predicted by the sum of $p\left(\mathrm{~d}_{i} \mid H\right)$ and $p\left(\mathrm{~d}_{i} \mid \sim H\right)$ inverse likelihood judgements, the posterior additivity scores were subjected to a one-way Analysis of Covariance with Participant as a factor and Inverse additivity as a covariate. The interaction term Participant $\times$ Inverse additivity did not reach statistical significance $[F(12,51)=1.54, \operatorname{MSE}=0.05, p=.14$, partial $\eta^{2}=.25$ ] indicating homogeneity of the individual regression lines. However, overall, the sum of complementary posterior probability judgements could not be directly inferred from the sum of their corresponding inverse likelihood judgements. Indeed the parameter estimates revealed that the $R^{2}$ coefficient was less than .80 , the confidence interval for the intercept $B$ coefficient did not include zero and the confidence interval for the covariate $B$ coefficient did not include one $\left(R^{2}=.43 ; B_{\text {Intercept }}=.29, \mathrm{CI}=[.11\right.$; $\left..47] ; B_{\text {Covariate }}=.69, \mathrm{CI}=[.51 ; .87]\right)$.

In sum, this shows that, overall, one could not assume that participants' complementary posterior probability judgements' direction and magnitude of deviations from unity could be explained by the direction and magnitude of the deviations from unity of those participants' inverse likelihood estimates. This result is not surprising since it was already found in the section on the inverse fallacy test that posterior probability judgements were generally not based on an evaluation of their corresponding inverse likelihood (see p. 107).

\section{Discussion of Experiment 2}

The aim of Experiment 2 was to further investigate whether individuals were committing the inverse fallacy when making posterior probability judgements, using a within-subjects design. Contrary to what was originally hypothesized, inverse likelihood judgements were not a better predictor of posterior probability judgements than representativeness estimates. In fact, the idiographic approach followed revealed that posterior probability judgements observed differed from one individual to the other with regards to the extent to which they were related to representativeness estimates, inverse likelihood estimates, subjective Bayesian values or even subjective base-rate neglect values. Overall, none of the existing accounts tested (inverse fallacy, representativeness heuristic, Base-Rate-Neglect-But-Otherwise-Bayesian algorithm and Bayesian 
algorithm) provided a general account of the processes underlying posterior probability judgements observed in Social judgement tasks.

In fact, different participants exhibited different strategies, which could rarely be unambiguously inferred from any of the judgements or values just mentioned. Indeed, out of 16 participants, only one participant could be unambiguously classified as using the representativeness heuristic. Moreover, all the participants whose posterior probability judgements could be reliably inferred from their corresponding inverse likelihood judgements (3 out 16) also exhibited judgements that could be reliably inferred from other predictors (either representativeness estimates or BRNBOB values). Consequently, it was impossible to infer what processes were underlying the posterior probability judgements observed.

Another issue investigated in this experiment was whether complementary posterior probability judgements were additive. Whereas median complementary judgements generally summed to about 1, an idiographic analysis based on individual posterior probability judgements instead of being based on averaged judgements revealed that individual judgements almost never added up to one. Out of the sixteen participants, only one made complementary judgements that consistently respected the additivity principle. This shows that additivity of posterior probability judgements in Socialjudgement tasks cannot be assumed, in line with what Baratgin \& Noveck (2000) already suggested. Consequential to the assumption that participants were committing the inverse fallacy when estimating their posterior probability judgements, the last hypothesis tested was that deviations of complementary posterior probability judgements from additivity could be predicted from the sum of their corresponding judgements of inverse likelihood. Again this hypothesis was not supported by the data. Yet this finding is not surprising since the test for the inverse fallacy had already shown that this strategy could not generally account for posterior probability judgements observed.

\section{CONCLUSIONS}

Using a process-focused approach to the study of posterior probability judgements, the aim of this study was to investigate the judgement methods underlying posterior probability judgement outputs observed in Social-judgement tasks; that is, judgements that an instance belongs to a category $H$ (rather than to a counterpart category $\sim H$ ), given that it is described by a description $D$. The review of the literature identified three 
alternative descriptive accounts. The base-rate neglect account (Kahneman \& Tversky, 1973) suggests that individuals neglect base-rate information when making posterior probability judgements; that is, they do not take the a priori proportions of instances in $H$ and $\sim H$ into account. The representativeness heuristic account (ibid.) suggests that individuals base their posterior probability judgements on the evaluation of the extent to which the instance described by $D$ is representative or typical of the members of the category $H$. The third account, referred to here as 'the inverse fallacy account' suggests that individuals are in fact estimating the inverse likelihood that an instance that belongs to a category $H$, will be described by a description $D$ (Gigerenzer \& Murray, 1987; Pollard \& Evans, 1983). Finally, a normative account is provided by Bayes's theorem, which prescribes that such posterior probability judgements be based on the ratio of the product of base rate and inverse likelihood for the target category to the sum of this product and the product of base rate and inverse likelihood for the alternative category.

The ultimate aim of the process-focused approach is to provide an algorithmic account of the behaviours observed, or in the present case, to explain how the task entails the application of a particular judgement method. For this reason, it is crucial to develop a reliable computational account of judgements (i.e., unambiguously identifying the judgement methods applied to solve the task) before we can work at understanding how such methods are triggered by the task and participants' cognitive processes.

The first study, based on a traditional nomothetic approach, revealed no effect of base-rate information on averaged posterior probability judgements. This finding, however, may have resulted from lack of a priori power to detect a mean difference between high and low base-rate conditions since such difference has customarily been observed to be small and substantially less than what would be prescribed by Bayes's theorem. In fact, tests for the Bayesian norm confirmed that averaged judgements significantly differed from Bayesian prescriptions, whether under high or low base-rate conditions. More closely related to the primary objective of this study was the test for the inverse fallacy account. In line with the hypothesis advanced, averaged posterior probability judgements were confounded with averaged inverse likelihood judgements. This suggested that the same processes may underlie both types of judgements and that the inverse fallacy was indeed a valid account of the processes underlying posterior probability judgements. Yet, whether individuals were using inverse likelihood estimates to make their posterior probability judgements could only be indirectly assessed by such 
a between-subjects design. Even a significant test for the base-rate neglect effect could not have led to the definite conclusion that all participants were indeed integrating base-rate information in their judgements.

The second experiment attempted to address this shortcoming by using a withinsubjects design and an idiographic approach to the analysis of the judgements observed. The measures collected allowed for testing whether each individual participant was indeed committing the inverse fallacy, relying on the representativeness heuristic, making Bayesian judgements or even solely neglecting base-rate information but otherwise computing the normative ratio of likelihoods. Additionally, it was possible to further investigate the extent to which individuals indeed respected the additivity principle. Surprisingly, results revealed that for the vast majority of participants (12 out of 16), individual judgement series could not be reliably inferred from any of these accounts. Out of the four remaining participants questioned, only one exhibited judgements that could unambiguously be attributed to the use of the representativeness heuristic. Two others exhibited judgements that could be accounted by either the inverse fallacy or the representativeness heuristic. And, a last participant exhibited judgements that were consistent with three accounts, namely, the representativeness heuristic, inverse fallacy and BRNBOB accounts. With regards to the additivity issue, results showed that although the sum of complementary median posterior probability judgements was generally close or equal to one, an idiographic analysis of the data revealed that only one out of the 16 participants who took part in the experiment systematically respected the additivity principle.

The implications of these results extend beyond normative considerations. The fact that participants do not respect the addivity principle entails that the complement to $p(H \mid D)$ judgements [i.e., $1-p(H \mid D)$ ] will not be representative of $p(\sim H \mid D)$ judgements. Accordingly, the validity of analyses involving both $p(H \mid D)$ and $p(\sim H \mid D)$ estimates, but based on the measure of $p(H \mid D)$ and the estimation of $p(\sim H \mid D)$ by the complement to $p(H \mid D)$ (e.g., Griffin \& Buehler, 1999) is undermined by the fact that people do not respect the additivity principle. Inferential tests revealed that neither the hypothesis that participants respected the additivity principle, nor the hypothesis that deviations of posterior probability judgements from additivity could be predicted from judgements of inverse likelihood were supported.

These results also point to the limitations of nomothetic approaches in inferring the processes underlying judgements. Conclusions that people neglect base-rate information 
or apply the representativeness heuristic in Social-judgement tasks invariably rely on averaged posterior probability judgements (see chap. III). Yet, the results of the present study clearly demonstrate that such inference from mean judgements to the general processes underlying individual judgements is likely to be unwarranted. Indeed, whereas averaged posterior probability judgements appeared to be indistinguishable from inverse likelihood judgements in Experiment 1, there was little evidence to suggest that most individuals are indeed committing the inverse fallacy when making posterior probability judgements, as shown in Experiment 2. Similarly, whereas median posterior probabilities for complementary categories may add up to one, the assumption that individuals respect the additivity principle was unjustified, as indicated by an idiographic analysis of complementary judgements in Experiment 2.

Yet again, even the idiographic approach followed in the second experiment was not enough to reliably infer the processes underlying judgements as posterior probability judgement outcomes matched outputs from competing accounts. This illustrates the limitations of inferring underlying processes from numerical estimates alone and calls for further research to identify the processes underlying posterior probability judgements. As mentioned earlier, it is only by developing a valid descriptive account of the strategies, heuristics, or methods used to solve these problems (i.e., identifying what individuals do) that it will be possible to further our understanding of the processes underlying those judgements (i.e., identifying how individuals do what they do). Unless a thorough computational account is available, the development of a sound algorithmic account will be somewhat constrained. The development of a computational account is also important from a normative perspective: a good understanding of the origin of observed deviations from normative principles should give better grounds for preventing the occurrence of such deviations.

Concerning the lack of support for the inverse fallacy account, this result suggests that the processes underlying posterior probability judgements in Social-judgement problems differ from those underlying such judgements in Textbook problems. In all likelihood, the suggestion that individuals may commit the inverse fallacy in Socialjudgement tasks was triggered by the common observation that individuals customarily evaluate the posterior probability $p(H \mid D)$ to be equal to the inverse likelihood $p(D \mid H)$ when the latter information is readily available in Textbook problems. Results from Experiment 2, however, demonstrated that the generalisation of this result to judgements observed in Social-judgement tasks was not warranted. 
The identification of the judgement methods applied in Social-judgement tasks may include the investigation of online verbal protocols (Ericsson \& Simon, 1993) or the manipulation of the relationship between inverse likelihood (the probability that $x$ has feature $D$ given that $x$ belongs to $H$ ) and representativeness. Tversky \& Kahneman (1982c) indeed suggested that where representativeness characterises the relationship between a category $H$ and an instance of that category described by $D$, this construct could either be defined as what is typical or modal, or else as what is prototypical; that is, what is "an ideal type that embodies the essence of the category" (Tversky \& Kahneman, 1982c, p. 86). They gave the example of two instances of the category "Frenchwoman" that may be considered representative of this category; namely, a "young, elegant Parisian" (ibid.) (prototypical instance) and a "chubby middle-aged woman from the provinces" (ibid.) (typical instance). Whereas both descriptions may be judged as highly representative of the category in question, one may conjecture that the two descriptions will lead to different likelihood judgements.

With regards to the observed deviations from additivity, the fact that the inverse fallacy account cannot provide an adequate explanation for those deviations is not surprising since the preceding results showed that posterior probability judgements in Social-judgements tasks were not, on the whole, determined by the commitment of the inverse fallacy. An alternative explanation might be suggested by Rottenstreich and Tversky's (1997) account of Support Theory. The authors indeed proposed that binary complementarity would only hold for judgements of $p(H)$ and $p(\neg H)$ where $\neg$ denote the explicit negation of hypothesis $H$ (e.g., 'not black' where $H$ is defined as 'black') but not for $p(H)$ and $p(\sim H)$ where $\sim$ denote the implicit negation of $H$ (e.g., 'white' where $H$ is also defined as 'black').

This suggests that $p(H \mid D)$ and $p(\neg H \mid D)$ judgements would add to 1 where $\neg H$ represents the explicit negation of $H$. By contrast, the authors suggested that $p(H \mid D)$ and $p(\sim H \mid D)$ judgements would not necessarily add to 1 where $\sim H$ represents the implicit negation of $H$. In the task used in this study, as in the standard lawyer-engineer problem, individuals are asked to estimate $p\left(H \mid D_{1}\right)$ and $p\left(\sim H \mid D_{1}\right)$, where $\sim H$ represents the set of individuals who are, e.g., accountants, and $D_{1}=(H \cap D) \cup(\sim H$ $\cap D)$; that is, $D_{1}$ represents the set of designers and accountants who are included in the set $D$ defining individuals described by description $d$. Yet, Rottenstreich and Tversky's (1997) argument also suggests that, when asked to evaluate $p\left(H \mid D_{1}\right)$, individuals are in 
fact considering the set $D_{2}$ defined by $(H \cap D) \cup(\neg H \cap D)$; that is, for example, the set of designers and non-designers who are described by description $d$. In this case, $D_{1} \subset$ $D_{2}$; that is, the set of designers and accountants who are described by $d$ is included in the set of designers and non-designers who are described by $d$. However, $D_{1} \neq D_{2}$; that is, the two sets are not equal since, presumably, there are individuals who are neither designers nor accountants but who are still described by $d$.

The multiplication law of probability (cf. chap. II, p. 15) dictates that,

$$
p(H \mid D)=\frac{p(H \cap D)}{p(D)} .
$$

Since, in the example used,

$$
\left(H \cap D_{1}\right)=\left(H \cap D_{2}\right)=(H \cap D),
$$

and,

$$
p\left(D_{1}\right)<p\left(D_{2}\right)
$$

it follows that,

$$
p\left(H \mid D_{1}\right)=\frac{p(H \cap D)}{p\left(D_{1}\right)}>p\left(H \mid D_{2}\right)=\frac{p(H \cap D)}{p\left(D_{2}\right)}
$$

Similarly, when asked to evaluate $p\left(\sim H \mid D_{1}\right)$, individuals are in fact considering the set $D_{3}$ defined by $(\sim H \cap D) \cup(\neg(\sim H) \cap D)$; that is, $D_{3}$ represents the set of accountants and non-accountants who are described by the specific description. Then, again, $D_{1} \subset D_{3}$ but $D_{1} \neq D_{3}$, and it follows that $p\left(D_{1}\right)<p\left(D_{3}\right)$ and, consequently,

$$
p\left(\sim H \mid D_{1}\right)>p\left(\sim H \mid D_{3}\right)
$$

Finally, if individuals are evaluating $p\left(H \mid D_{2}\right)$ and $p\left(\sim H \mid D_{3}\right)$ instead of $p\left(H \mid D_{1}\right)$ and $p\left(\sim H \mid D_{1}\right)$, then,

$$
p\left(H \mid D_{1}\right)+p\left(\sim H \mid D_{1}\right)>p\left(H \mid D_{2}\right)+p\left(\sim H \mid D_{3}\right)
$$

or else, since $p\left(H \mid D_{1}\right)+p\left(\sim H \mid D_{1}\right)=1$, one should expect that the sum of judgements observed would be superadditive, that is, to add up to less than 1 .

Thus, a potential explanation for the deviations from the additivity principle observed could be that people are bringing in real world knowledge and calibrate their judgements accordingly. In particular, following Rottenstreich and Tversky's (1997) argument, one could propose that participants rely on the set of non-designers, which is larger than the set of accountants. Yet, it follows from this explanation that complementary judgements should be systematically superadditive. Yet, this conclusion is at odds with observed results as individual plots for $p(H \mid D)$ and $p(\sim H \mid D)$ judgements 
revealed that only 3 out of the sixteen individuals (\#7, \#13, and \#16) who took part in the experiment consistently provided superadditive estimates (see Figure IV-12, p. 122).

Altogether, these results suggest that more research is needed to unambiguously identify the judgement methods that underlie outputs observed in Social-judgement tasks and their deviation from normative principles. The development of such an algorithmic account would in turn allow assessing the psychological plausibility of existing algorithmic accounts whose purpose is to explain how such computations are carried out. For example, a new algorithmic account has recently been developed to account for the judgements observed in Social-judgement tasks in terms of memory processes (Dougherty, Gettys, \& Ogden, 1999). Broadly speaking, this model (called MDM or MINERVA-DECISION-MAKING) assumes that computations are based on (less than perfect) retrieval of frequencies of observations. This account is based on Hintzman's (1984, 1988, as cited in Dougherty et al., 1999) MINERVA model. The authors assessed the predictive value of the MDM model by comparing averaged simulated outputs with Bayesian computations. The results observed with the simulations were consistent with what is generally observed in Social-judgement studies (Dougherty et al., 1999).

Such an assessment, however, is not without shortcomings. First, it relies on the assumption that posterior probability judgements in Social-judgement tasks are indeed based on memory traces of past frequencies of observation. As already mentioned (see chap. II), there are other possible bases of probability assessments (i.e., stereotypical beliefs, pro and con reasons, etc.). Second, the predictive value of the MDM model in its current state cannot account for individual differences (i.e., it predicts aggregated outcomes). Therefore, the computational account upon which the MDM model is based still needs to be validated and its idiographic predictive value assessed, before the MDM model can be taken as a plausible algorithmic account of the computational processes underlying posterior probability judgements in Social-judgement tasks.

In sum, more research is thus needed within the Social-judgement paradigm before the judgement methods underlying observed outputs can be identified (computational level of analysis) and before the cognitive processes underpinning the elicitations of such judgement methods can be established with force (algorithmic level of analysis). The purpose of this thesis, however, was to bring together results from the three experimental paradigms within which posterior probability judgements are investigated. 
Accordingly, the next chapter will address issues similar to those investigated here, but within the Textbook-problem paradigm.

Ske 

his chapter will elaborate on the process-focused study of posterior probability judgements within the Textbook-problem paradigm. This paradigm differs from the Social-judgement paradigm with regards to the type of information that serves as a basis for the posterior probability judgements elicited as well as with regards to the explanations proposed to explain the judgement outputs observed. Social Judgement tasks only provide the base-rate information [i.e., $p(H)$ and $p(\sim H)$ ] in a numerical form. Likelihood information [i.e., $p(D \mid H)$ and $p(D \mid \sim H)$ ] can only be subjectively assessed on the basis of a verbal description, which represents the datum $D$. In contrast, Textbook problems present participants with numerical information for all the components of Bayes's theorem [i.e., $p(H), p(\sim H), p(D \mid H)$, and $p(D \mid \sim H)]$.

The primary aim of the process-focused study of posterior probability judgements is the development of a sound computational account of the judgement methods that lead to the judgement outputs observed. As with the Social-judgement paradigm, the literature review (see chap. III) identified several potential computational accounts. In contrast with the Social-judgement paradigm, however, the representativeness heuristic was dismissed either as an explanatory or even a computational account of the processes underlying observed judgement outcomes in Textbook problems (e.g., Kahneman \& Tversky, 1980; Bar-Hillel, 1990), suggesting that different processes may be at work in Textbook problems.

The predominant computational account of the processes underlying posterior probability judgements observed within the Textbook-problem paradigm was once identified as the base rate neglect account (e.g., see Bar-Hillel, 1980; Lyon \& Slovic, 1976; Tversky \& Kahneman, 1982a; see Koehler, 1996, for a review) or the tendency to ignore base-rate information [i.e., $p(H)$ and $p(\sim H)$ information] when evaluating posterior probability judgements. Yet, the base-rate neglect account describes what people may not do, not what actually underlies their judgements. An alternative computational account identified by the review was the inverse fallacy account (Koehler, 1996). The possibility that people may rely on the inverse likelihood $p(D \mid H)$ when 
estimating the posterior probability $p(H \mid D)$ within Social-judgement studies was merely suggested (e.g., see Gigerenzer \& Murray, 1987). In contrast, with Textbook problems, there is empirical evidence available to show that most individuals indeed tend to rely on the numerical value of $p(D \mid H)$ provided in the text, when evaluating $p(H \mid D)$ in Textbook problems (see, e.g., Bar-Hillel, 1980; Hamm, 1993, 1994; Tversky \& Kahneman, 1980).

Therefore, a first objective of this study was to distinguish between the base-rate neglect and the inverse fallacy account, and empirically identify which of these two computational accounts could describe what judgement methods were applied to produce the posterior probability judgement outputs observed in Textbook problems. One way of distinguishing between base-rate neglect and inverse fallacy is to study posterior probability judgements when base rates are equal, i.e., when $p(H)=p(\sim H)$. In this particular situation, base-rates need not be included for the computation of Bayesian posterior probabilities. Indeed, where $H$ and $\sim H$ denote two mutually exclusive and collectively exhaustive hypotheses, and D represents a datum, Bayes's theorem can be written as:

$$
p(H \mid D)=\frac{p(H) \cdot p(D \mid H)}{p(H) \cdot p(D \mid H)+p(\sim H) \cdot p(D \mid \sim H)} .
$$

Since $p(H)=p(\sim H)$, equation (V. 1) can be rewritten as,

$$
p(H \mid D)=\frac{p(H) \cdot p(D \mid H)}{p(H) \cdot p(D \mid H)+p(H) \cdot p(D \mid \sim H)} .
$$

By factorising $p(H)$, equation (V. 2) becomes,

$$
p(H \mid D)=\frac{p(H)}{p(H)} \times \frac{p(D \mid H)}{p(D \mid H)+p(D \mid \sim H)} .
$$

Finally, after simplification, equation (V. 3) becomes,

$$
p(H \mid D)=\frac{p(D \mid H)}{p(D \mid H)+p(D \mid \sim H)} .
$$

Thus when base-rates are equal, participants who merely neglect base-rate information but otherwise compute the normative ratio of inverse likelihoods would exhibit Bayesian judgements. However, if participants are indeed committing the inverse fallacy [i.e., equate $p(H \mid D)$ with $p(D \mid H)$ ], then their posterior probability judgements $p(H \mid D)$ should vary as a function of the corresponding inverse likelihood values $p(D \mid H)$ provided. 
To identify whether individuals neglect base-rate information or whether they indeed commit the inverse fallacy (which entails both the neglect of the base-rate information and the neglect of the $p(D \mid \sim H)$ likelihood information) is an important issue from a computational standpoint: unless the judgement methods underlying the judgement outcomes observed can be positively identified, little progress can be made concerning the development of an algorithmic account explaining why such methods were elicited by the task. Additionally, knowledge of the judgement methods applied can help to explain and therefore predict the direction and the magnitude of deviations from normative principles such as the Bayesian norm or the additivity principle (see chap. II, p. 16).

The review of the literature indeed revealed that there is no consensus within the literature as to whether probability judgements of complementary hypotheses should be expected to be additive (see chap. III, p. 47). With specific regards to posterior probability judgements, however, if Textbook problems lead individuals to commit the inverse fallacy [that is, if they judge posterior probability $p(H \mid D)$ to be equal to its corresponding inverse likelihood value $p(D \mid H)$ and posterior probability $p(\sim H \mid D)$ to be equal to its corresponding inverse likelihood value $p(D \mid \sim H)]$ then deviations from the additivity principle [i.e., deviations of the sum $p(H \mid D)+p(\sim H \mid D)$ from 1] should be expected when the sum of inverse likelihood values [i.e., the sum $p(D \mid H)+p(D \mid \sim H)$ ] does not add up to 1 . Moreover, the direction [i.e., the superadditivity or subadditivity of the sum $p(H \mid D)+p(\sim H \mid D)$ ] and magnitude [i.e., the extent to which the sum $p(H \mid D)+p(\sim H \mid D)$ deviates from 1] of these deviations should depend on the direction and magnitude of the deviations from additivity of the sum of the corresponding inverse likelihood values [i.e., the sum $p(D \mid H)+p(D \mid \sim H)]$. The second objective of this study was therefore to test whether a manipulation of the inverse likelihood values provided, $p(D \mid H)$ and $p(D \mid \sim H)$, would affect the observed deviations of complementary posterior probability judgements from additivity.

A scenario adapted from Slowiaczek et al. (1992) was used. First, an experiment (Experiment 3 of this thesis) was designed to discriminate between base-rate neglect and inverse fallacy. A key objective of this experiment was to test the hypothesis that observed deviations from Bayesian prescriptions would vary as a function of the values of inverse likelihood presented, thereby positing that individuals commit the inverse fallacy, rather than merely neglecting base-rate information, when making posterior probability judgements. A second objective was to test whether the sum of 
complementary posterior probability estimates [i.e., the sum of $p(H \mid D)$ and $p(\sim H \mid D)$ judgements] would vary as a function of the sum of their corresponding inverse likelihood [i.e., the sum of $p(D \mid H)$ and $p(D \mid \sim H)$ ].

Following the previous observation that the proportion of inverse and Bayesian judgements was lower than what is usually observed, the last objective of this study was to examine whether the number of task goals [i.e., the prompt for both $p(H \mid D)$ and $p(\sim H \mid D)$ judgements vs. the prompt for either $p(H \mid D)$ judgements or $p(\sim H \mid D)$ judgements, but not both] would affect the proportion of participants committing the inverse fallacy or even providing Bayesian judgements.

Consequently, another experiment (Experiment 4) was designed to test the prediction that individuals will be more likely to commit the inverse fallacy when they are asked to evaluate both $p(H \mid D)$ and $p(\sim H \mid D)$ at once, rather than $p(H \mid D)$ alone. To test this hypothesis, the task presentation was manipulated by varying administration procedures. Participants were given the same series of problems, but the measures of $p(H \mid D)$ and $p(\sim H \mid D)$ were no longer made together in sequence. Rather than being asked to estimate both $p(H \mid D)$ and $p(\sim H \mid D)$ for a number of stimuli (as in Experiment 3), a first group of participants were asked to make $p(H \mid D)$ judgements for a set of stimuli. A second group of participants were again presented with the same set of stimuli but, this time, were asked to make $p(\sim H \mid D)$ judgements. This manipulation was expected to lead more participants to aggregate $p(D \mid H)$ and $p(D \mid \sim H)$ values in judging $p(H \mid D)$. Accordingly, the proportion of participants committing the inverse fallacy was expected to decrease when complementary posterior probability judgements were no longer made together in sequence, and the proportion of participants providing Bayesian estimates was expected to increase, although it was expected that the majority of participants would still provide judgements that were not Bayesian.

The remainder of this chapter gives a detailed presentation of each of the two experiments conducted within the Textbook-problem paradigm and their corresponding results. The implications of those results for the process-focused study of posterior probability judgements within this paradigm will be discussed in the final section of this chapter. 


\section{EXPERIMENT 3}

This experiment examined whether evidence for the inverse fallacy emerged even when base rate neglect could be ruled out. The problem used was adapted from Slowiaczek et al. (1992, Experiment 1A). This problem presented a task in which participants were asked to estimate the posterior probability that an encountered alien creature was one of two mutually exclusive types in light of the presence or absence of a diagnostic feature. Skov and Sherman (1986) noted that the use of natural groups imposes restrictions on the likelihood of a particular feature in these groups. In such cases, diagnosticity and likelihood are often confounded: a diagnostic trait (e.g. "likes parties") would be frequent in the focal group (e.g., extroverts) and infrequent in the alternative group (introverts). Thus, the use of unnatural categories allowed controlling for the likelihood of the features.

The first hypothesis was that a majority of participants would commit the inverse fallacy; that is, their $p(H \mid D)$ judgements to be equal to the $p(D \mid H)$ values provided in the text. Secondly, (and directly following from the first hypothesis), it was hypothesized that the deviation between participants' $p(H \mid D)$ judgements and Bayesian values [denoted $\left.p^{*}(H \mid D)\right]$, could be predicted by the deviation between $p(D \mid H)$ and $p^{*}(H \mid D)$. Bayesian values $\left[p^{*}(H \mid D)\right]$ were calculated by applying Bayes's theorem, using the values of $p(D \mid H)$ and $p(D \mid \sim H)$ provided in the text. Thus, since participants were expected to judge $p(H \mid D)$ to be equal to the $p(D \mid H)$ values provided in the text, their judgements were also expected to overestimate $p^{*}(H \mid D)$ when $p(D \mid H)>p^{*}(H \mid D)$ and underestimate $p^{*}(H \mid D)$ when $p(D \mid H)<p^{*}(H \mid D)$. Moreover, by experimentally manipulating the size of the deviation between $p^{*}(H \mid D)$ and $p(D \mid H)$, it was also possible to predict the magnitude of participants' posterior probability judgement deviations from Bayesian values. The third hypothesis tested concerned the additivity issue, and was also a direct consequence of individuals committing the inverse fallacy. In particular, assuming that individuals' posterior probability judgements of $p(H \mid D)$ and $p(\sim H \mid D)$ would be equal to $p(D \mid H)$ and $p(D \mid \sim H)$, respectively, it was expected that when the $p(D \mid H)$ and $p(D \mid \sim H)$ values provided added up to less than one, the sums of participants' $p(H \mid D)$ and $p(\sim H \mid D)$ judgements would be superadditive (i.e., they would also add up to less than one). Conversely, the sums of their judgements of $p(H \mid D)$ and $p(\sim H \mid D)$ were expected to be subadditive (i.e., to add to more than one) when the sum of $p(D \mid H)$ and $p(D \mid \sim H)$ exceeded one. 
The experimental design also allowed distinguishing between the inverse fallacy and a simpler, matching heuristic. In the domain of logical reasoning, Evans (1998) defined a matching bias as a tendency to only consider as relevant the information whose lexical content matches that of the information presented in the propositional rule to be tested. By extension, the tendency to equate $p(H \mid D)$ with $p(D \mid H)$ could be defined as the tendency to estimate $p(H \mid D)$ on the basis of the match between the lexical content of the experimental question (e.g., 'What is the probability that $x$ is $H$ given that $x$ has D?') and the information presented in the likelihood information (e.g., 'The probability that $x$ has $D$ given that $x$ is $H$ is $60 \%$ '). But consider the case where people are still presented with $p(D \mid H)$ and $p(D \mid \sim H)$ information but are asked to estimate $p(H \mid \neg D)$ (e.g., 'What is the probability that $x$ is $H$ given that $x$ does not have $D$ '). If they were following a simple matching strategy, one would expect that they would evaluate $p(H \mid \neg D)$ to be equal to the value of $p(D \mid H)$. This latter piece of information indeed remains the information whose lexical content matches (if only to some extent) that of the experimental question. By contrast, the inverse fallacy account proposes that people will estimate $p(H \mid \neg D)$ with the value of its corresponding inverse likelihood $p(\neg D \mid H)$. Consequently, when presented with the value of $p(D \mid H)$ and $p(D \mid \sim H)$, and when asked to estimate $p(H \mid \neg D)$, if participants were committing the inverse fallacy, one would expect them to judge $p(H \mid \neg D)$ to be equal to $1-p(D \mid H)$ whereas if their judgements reflected a matching bias, one would expect them to equate their $p(H \mid \neg D)$ judgement with the value of $p(D \mid H)$ provided in the text. The distinction between inverse and matching heuristics was therefore possible in cases where participants were presented with $p(D \mid H)$ and $p(D \mid \sim H)$ values and asked to estimate $p(H \mid \neg D)$.

\section{Method}

\section{Participants}

Forty-five University of Hertfordshire undergraduates ( 43 women and 2 men, mean age $=23.4$ years, age range $=18-50$ ) participated in the experiment for course credit. They were tested individually.

\section{Materials and Procedure}

All participants were provided with the same 13-page questionnaire. The first page presented their task as follows: 
Imagine you are visiting a planet called Vuma. There are two and only two types of invisible creatures that live on this planet. There are 1 million Gloms, and 1 million Fizos.

You will randomly meet 12 creatures. Imagine you are particularly interested in guessing their identity. Each time you meet one of the invisible creatures, you want to know whether it is a Glom or a Fizo. You will walk with an interpreter who will ask each creature whether or not it possesses a certain feature. Each time, you will be provided with the percentages of Gloms and Fizos on Vuma possessing the target feature. The creatures cannot help but to tell the truth, so you can be sure you will get a truthful answer which will provide you with some information about the creature's identity.

Participants then were provided with an example and were told: "On the basis of the creature's answer, you will be asked to estimate both the likelihood that it is a Glom and the likelihood that it is a Fizo. Turn the page for your first encounter." Participants "met" 12 creatures one by one on the subsequent pages of the questionnaires. For each of these 12 encounters, the creature was asked about a different feature and participants were provided with a reminder of the number of Gloms and Fizos on the planet as well as the percentages of each type of creature that possessed the feature. In order to be consistent with Slowiaczek et al. (1992), posterior probability judgements were elicited using a frequency question and participants were asked to estimate the 'chances in 100' rather than the 'probability' that the creature was a Glom or that the creature was a Fizo. A summary of the stimuli is presented in the first three columns of Table V-1. Figure $\mathrm{V}-1$ shows a template of the questionnaire layout.

\begin{tabular}{|c|c|}
\hline $\begin{array}{l}\text { Gloms } \\
\text { (I million) }\end{array}$ & $\begin{array}{l}\text { Fizos } \\
\text { ( } \text { million) }\end{array}$ \\
\hline $98 \%$ play the harmonica & $58 \%$ play the harmonica \\
\hline \multicolumn{2}{|c|}{$\begin{array}{l}\text { You meet a creature and the interpreter translates } \\
\text { the following conversation: } \\
\text { Interpreter: "Do you play the harmonica?" } \\
\text { Creature: "Yes, I do!" }\end{array}$} \\
\hline \multicolumn{2}{|c|}{$\begin{array}{l}\text { On the basis of the creature's response, answer the } \\
\text { following questions: }\end{array}$} \\
\hline \multicolumn{2}{|c|}{$\begin{array}{l}\text { 1) Please estimate the chances in } 100 \text { that this creature is } \\
\text { a Glom. }\end{array}$} \\
\hline \multicolumn{2}{|c|}{$\begin{array}{l}\text { There are ___ chances in } 100 \text { that this creature } \\
\text { is a Glom. }\end{array}$} \\
\hline \multicolumn{2}{|c|}{$\begin{array}{l}\text { 2) Please estimates the chances in } 100 \text { that this } \\
\text { creature is a Fizo. }\end{array}$} \\
\hline \multicolumn{2}{|c|}{$\begin{array}{l}\text { There are ___ chances in } 100 \text { that this creature } \\
\text { is a Fizo. }\end{array}$} \\
\hline
\end{tabular}

Figure V-1 - Template of the questionnaire layout used in Experiment 3. 
Table V-1

Proportion of Gloms and Fizos presenting each of twelve features, encountered creature's response, and corresponding inverse and Bayesian values.

\begin{tabular}{lcccccc}
\hline & \multicolumn{2}{c}{ Proportions } & \multicolumn{2}{c}{ Inverse values } & \multicolumn{2}{c}{ Bayesian values } \\
\cline { 2 - 7 } \multicolumn{1}{c}{ Features } & Gloms & Fizos & $p(D \mid H)$ & $p(D \mid \sim H)$ & $p^{*}(H \mid D)$ & $p^{*}(\sim H \mid D)$ \\
\hline & \multicolumn{7}{c}{ 'Yes' creature's response } & & \\
$\mathrm{f}_{1}$ (plays the harmonica) & $98 \%$ & $58 \%$ & .98 & .58 & .63 & .37 \\
$\mathrm{f}_{2}$ (wears hula hoops) & $90 \%$ & $50 \%$ & .90 & .50 & .64 & .36 \\
$\mathrm{f}_{3}$ (has a flying license) & $80 \%$ & $40 \%$ & .80 & .40 & .67 & .33 \\
$\mathrm{f}_{4}$ (smokes maple leaves) & $42 \%$ & $2 \%$ & .42 & .02 & .96 & .04 \\
$\mathrm{f}_{5}$ (has gills) & $50 \%$ & $10 \%$ & .50 & .10 & .83 & .17 \\
$\mathrm{f}_{6}$ (breeds scampi) & $60 \%$ & $20 \%$ & .60 & .20 & .75 & .25 \\
\hline & & No' creature's response & & & \\
$\mathrm{f}_{7}$ (exhales fire) & $2 \%$ & $42 \%$ & .98 & .58 & .63 & .37 \\
$\mathrm{f}_{8}$ (gurgles a lot) & $10 \%$ & $50 \%$ & .90 & .50 & .64 & .36 \\
$\mathrm{f}_{9}$ (gulp bluebottles down) & $20 \%$ & $60 \%$ & .80 & .40 & .67 & .33 \\
$\mathrm{f}_{10}$ (drinks petrol) & $58 \%$ & $98 \%$ & .42 & .02 & .96 & .04 \\
$\mathrm{f}_{11}$ (eats iron ore) & $50 \%$ & $90 \%$ & .50 & .10 & .83 & .17 \\
$\mathrm{f}_{12}$ (climbs walls) & $40 \%$ & $80 \%$ & .60 & .20 & .75 & .25 \\
\hline
\end{tabular}

Note. $D$ denotes $\mathrm{d}$ for 'yes' creature's responses and $\neg \mathrm{d}$ for 'no' creature's responses. $H$ denotes the hypothesis 'Creature $x$ is a Glom' and $\sim H$, the hypothesis 'Creature $x$ is a Fizo.' For 'no' responses, inverse values correspond to the complement to one of the proportions presented.

\section{Stimuli and design}

The stimuli thus each represented one of twelve probability problems involving a feature $\mathrm{f}_{i}\left(i=1\right.$ to 12 ; e.g., $\mathrm{f}_{1}=$ 'plays the harmonica', see Table $\left.\mathrm{V}-1\right)$ and two collectively exhaustive and mutually exclusive hypotheses, namely $H=$ 'creature $x$ is a Glom' and $\sim H=$ 'creature $x$ is a Fizo.' Base-rates were always identical [i.e., $p(H)=$ $p(\sim H)=.50$ ] since Gloms and Fizos creatures were said to be represented in equal proportions. The creature's response indicated whether or not it possessed feature $f_{i}$ and determined the type of posterior probability judgement elicited. A 'yes' response called for the judgement of the posterior probabilities $p(H \mid d)$ and $p(\sim H \mid d)$ whereas a 'no' response called for the judgement of the posterior probabilities $p(H \mid \neg \mathrm{d})$ and $p(\sim H \mid \neg \mathrm{d})$. For 'yes' stimuli, the proportion of Gloms having the feature $\mathrm{f}_{i}$ thus denoted the inverse likelihood $p\left(\mathrm{~d}_{\mathrm{i}} \mid \mathrm{H}\right)$ and the proportion of Fizos having the same feature denoted the inverse likelihood $p\left(\mathrm{~d}_{\mathrm{i}} \mid \sim \mathrm{H}\right)$. For 'no' stimuli, the inverse likelihoods 
$p\left(\neg \mathrm{d}_{i} \mid H\right)$ and $p\left(\neg \mathrm{d}_{i} \mid \sim \mathrm{H}\right)$ corresponded to the complement to one of the stated proportions of Gloms and Fizos having the feature $\mathrm{f}_{i}$, respectively.

For each of the 12 stimuli used, posterior probability measures corresponded to distinct Bayesian values and distinct inverse values. Those values are reported in the last four columns of Table $\mathrm{V}-1$. The $p(D \mid H)$ and $p(D \mid \sim H)$ values presented with 'no' stimuli (i.e., when $D=\neg \mathrm{d}$ ) were chosen so that the creature's response would have no effect on participants' judgements, whether those judgements were based on inverse values or Bayesian computations. Thus, the set of 'no' stimuli were perfectly matched to the set of 'yes' stimuli in terms of the Bayesian and inverse values (see Table V-1).

Based on the assumption that participants would commit the inverse fallacy and equate posterior probability judgements with their corresponding inverse values, deviations between posterior probability judgements and Bayesian values were expected to vary as a function of the deviations between inverse values and Bayesian values. Similarly, the deviations from additivity of the sum of complementary posterior probability judgements were expected to vary as a function of the sum of their corresponding inverse values. Each of the 12 encounters thus represented a unique stimulus condition in a 2 (conditioning datum: $D=\mathrm{d}, D=\neg \mathrm{d}) \times 2$ (expected direction of deviation: overestimation, underestimation) $\times 3$ (expected magnitude of deviation: small, medium, large) fully-crossed repeated-measures design with two dependent variables measured for each stimulus: $p(H \mid D)$ and $p(\sim H \mid D)$. The expected deviations between posterior probability judgements and Bayesian values are summarised in Table V-2. The magnitudes of overestimation and underestimation of Bayesian values were determined by the difference between inverse values and Bayesian values presented in Table V-1. The expected deviations between the sum of complementary posterior probability judgements and additivity are summarised in Table V-3. Expected magnitudes of superadditivity and subadditivity were determined by adding $p(D \mid H)$ and $p(D \mid \sim H)$ values. The order of stimulus presentation was randomised for each participant (each participant was presented with the stimuli in one of 12 different presentation order), and question order was counterbalanced across participants. 
Table V-2

Expected deviations of posterior probability judgements from Bayesian benchmarks as a function of the magnitude and direction of the deviation of inverse values from Bayesian values for both $p(H \mid D)$ and $p(\sim H \mid D)$ measures.

\begin{tabular}{lccc}
\hline & \multicolumn{3}{c}{ Magnitude } \\
\cline { 2 - 4 } Measure & Large & Medium & Small \\
\hline \multirow{4}{*}{ Overestimation } \\
$p(H \mid D)$ & 0.35 & 0.26 & 0.13 \\
$p(\sim H \mid D)$ & 0.21 & 0.14 & 0.07 \\
\hline & Underestimation & \\
$p(H \mid D)$ & -0.54 & -0.33 & -0.15 \\
$p(\sim H \mid D)$ & -0.07 & -0.05 & -0.02 \\
\hline
\end{tabular}

Note. $D$ represents either $\mathrm{d}$ or $\neg \mathrm{d}$ as the conditioning event was not expected to affect observed deviations. Expected deviations from Bayesian benchmarks were computed by subtracting Bayesian values from their corresponding inverse values.

Table V-3

Expected deviations of the sum of complementary posterior probability judgements [i.e., $p(H \mid D)+p(\sim \mathrm{H} \mid D)]$ from additivity as a function of the magnitude and direction of the deviation of the sum of corresponding inverse values from 1.

\begin{tabular}{cccc}
\hline & \multicolumn{3}{c}{ Magnitude } \\
\cline { 2 - 4 } Direction & Large & Medium & Small \\
\hline Subadditivity & 1.56 & 1.4 & 1.2 \\
Superadditivity & 0.44 & 0.6 & 0.8 \\
\hline
\end{tabular}

Note. $D$ represents either $\mathrm{d}$ or $\neg \mathrm{d}$ as the conditioning event was not expected to affect observed deviations. Expected deviations from additivity were computed by adding $p(D \mid H)$ and $p(D \mid \sim H)$ values.

\section{Results}

\section{Classification of estimates}

The first hypothesis tested in this experiment was that the majority of participants would commit the inverse fallacy when making their posterior probability judgements. In order to test whether participants committed the inverse fallacy, each single judgement was compared to the value of its corresponding inverse and Bayesian probability, respectively. An estimate was classified as Bayesian or inverse if it was equal to its 
corresponding Bayesian or inverse value, respectively, within a margin of error of \pm .02 . In similar attempts to classify participants' estimates, other authors have chosen stricter criteria of classification. For instance, Gigerenzer and Hoffrage (1995) classified a given estimate as Bayesian if it was equal to the value of Bayesian probability, with a margin of error of $\pm 1 \%$. However, the present choice of a margin of error of \pm .02 results in only 5 out of 100 possible values potentially classified as Bayesian, assuming that judgements are made on a scale ranging from 1 to 100 and are always given in an integer form. Hence, the Type I error of wrongly classifying a judgement as Bayesian is set at the usual .05 rate. Figure V-2 shows the distribution of participants according to the number of their estimates classified as Bayesian or inverse, for both types of judgements collected [i.e., $p(H \mid D)$ and $p(\sim H \mid D)$ judgements]. Overall, 91\% of the 45 series of $12 p(H \mid D)$ judgements matched a Bayesian value on less than four out of 12 occasions. By contrast, half of those 45 series of 12 judgements matched an inverse value on at least 10 out of 12 occasions. This result plainly demonstrates the prevalence of the inverse fallacy in posterior probability judgements.

Also, as expected, participants did not rely on a matching heuristic since most estimates of $p(H \mid \neg \mathrm{d})$ and $p(\sim H \mid \neg \mathrm{d})$ were estimated with inferred values for $p(\neg \mathrm{d} \mid H)$ and $p(\neg \mathrm{d} \mid \sim H)$, respectively. Within the set of 'no' stimuli, 55\% of participants provided at least 7 (out of 12) estimates equal to the inverse value whereas only one participant provided 7 estimates consistent with a matching heuristic. A Wilcoxon matched-pairs signed-ranks test was used to test the hypothesis that the number of inverse matches and the number of Bayesian matches had different distributions. The different intervals of numbers of matches $(0,1-3,4-6,7-9$, and 10-12) were given a score of 1 to 5 , respectively. For both $p(H \mid D)$ and $p(\sim H \mid D)$ judgements, the distributions of inverse and Bayesian matches significantly differed from each other at a $0.025 \alpha$-level, to protect the analysis against inflated Type I errors. Table V-4 summarises the test statistics. 

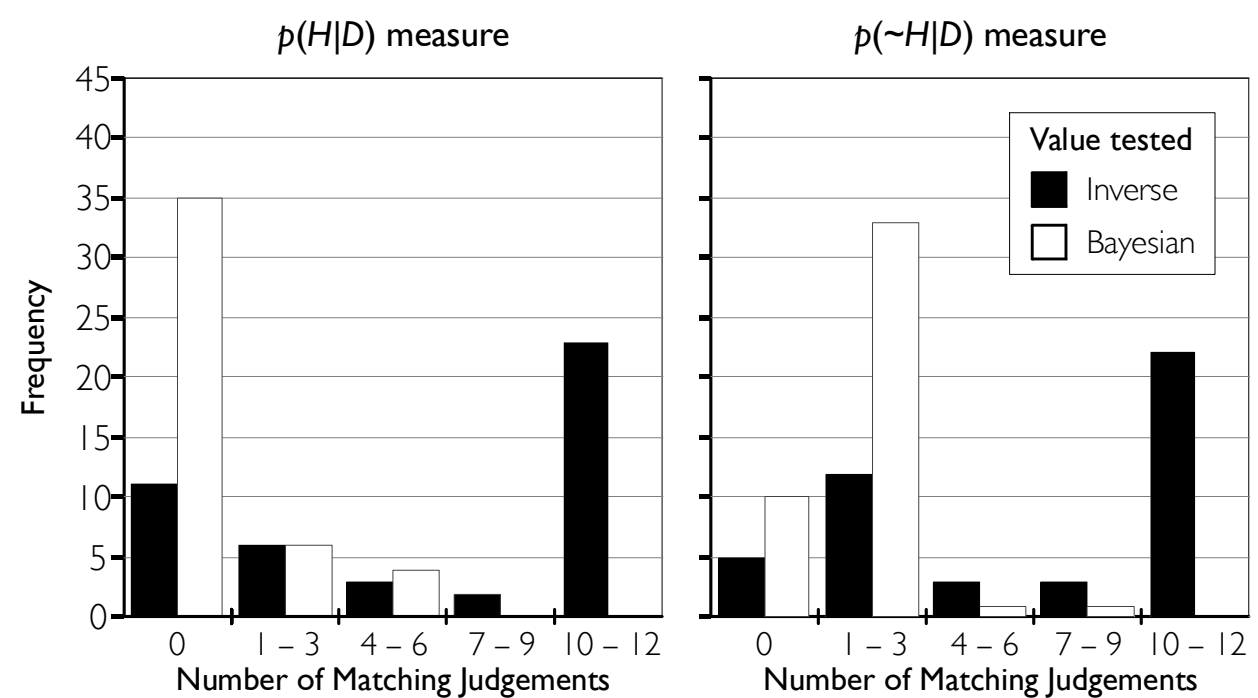

Figure $V-2$ - Frequency of different number of judgements matching the value tested (Bayesian, Inverse) as a function of type of measure $[p(H \mid D), p(\sim H \mid D)]$.

Note. $N=45$ for both $p(H \mid D)$ and $p(\sim H \mid D)$ judgements.

Table V-4

Wilcoxon's matched-pairs signed-ranks test statistics for the comparison of the distribution Bayesian and Inverse number of matches, for each type of judgement elicited.

\begin{tabular}{rrcrc}
\hline & \multicolumn{3}{c}{ Judgements } \\
& \multicolumn{3}{c}{$p(H \mid D)$} & \multicolumn{2}{c}{$p(\sim H \mid D)$} \\
\cline { 2 - 5 } & $N$ & Sum of Ranks & $N$ & Sum of Ranks \\
\hline Negative Ranks $^{\mathrm{a}}$ & 33 & 790.00 & 36 & 874.50 \\
Positive Ranks $^{\mathrm{b}}$ & 8 & 71.00 & 7 & 71.50 \\
Ties $^{\mathrm{c}}$ & 4 & & 2 & \\
$z$ & & $-4.68^{*}$ & & $-4.87^{*}$ \\
\hline
\end{tabular}

"p $<.025$

${ }^{a}$ No. of Bayesian matches $<$ No. of Inverse matches.

${ }^{b}$ No. of Bayesian matches $>$ No. of Inverse matches.

${ }^{\mathrm{c}}$ No. of Bayesian matches $=$ No. of Inverse matches.

\section{Deviations from Bayesian judgement}

The second hypothesis of this experiment stated that participants' posterior probability judgement deviations from Bayesian values would be predicted by the deviation between the inverse and Bayesian values. To test for this hypothesis, participants' $p(H \mid d), p(H \mid \neg d), p(\sim H \mid d)$ and $p(\sim H \mid \neg d)$ judgements were converted to deviation scores $\Delta$ by subtracting their corresponding Bayesian value [i.e., $p^{*}(\mathrm{H} \mid \mathrm{d})$, $p^{*}(\mathrm{H} \mid \neg \mathrm{d}), p^{*}(\sim \mathrm{H} \mid \mathrm{d})$ and $\left.p^{*}(\sim \mathrm{H} \mid \neg \mathrm{d})\right]$ for each of the six combinations of direction and magnitude of expected deviation from the Bayesian norm. Thus, $\Delta=0$ for accurate 
Bayesian estimates, $\Delta>0$ for overestimations of Bayesian probabilities, and $\Delta<0$ for underestimations of Bayesian probabilities. Analyses including order of stimulus presentation and order of question presentation as between-subject variables revealed no significant effect of these manipulations and the data were collapsed across order.

Table V-5 shows the mean deviation scores and standard errors obtained in each of the conditions and for each of the two measures, $p(H \mid D)$ and $p(\sim H \mid D)$.

Table V-5

Mean deviation scores from Bayesian prescriptions as a function of expected direction of deviation, expected magnitude of deviation and conditioning datum, for each of the two posterior probability measures collected.

\begin{tabular}{|c|c|c|c|c|c|c|}
\hline \multirow{5}{*}{$\begin{array}{l}\text { Conditioning } \\
\text { datum }\end{array}$} & \multicolumn{6}{|c|}{ Direction } \\
\hline & \multicolumn{3}{|c|}{ Overestimation } & \multicolumn{3}{|c|}{ Underestimation } \\
\hline & \multicolumn{3}{|c|}{ Magnitude } & \multicolumn{3}{|c|}{ Magnitude } \\
\hline & Small & Medium & Large & Small & Medium & Large \\
\hline & $M(S E)$ & $M(S E)$ & $M(S E)$ & M (SE) & $M(S E)$ & M (SE) \\
\hline \multicolumn{7}{|c|}{$p(H \mid D)$ judgements } \\
\hline $\mathrm{d}$ & $.05(.02)$ & $.14(.03)$ & $.20(.03)$ & $-.20(.02)$ & $-.33(.03)$ & $-.53(.03)$ \\
\hline$\neg \mathrm{d}$ & $-.04(.04)$ & $.06(.05)$ & $.08(.06)$ & $-.23(.02)$ & $-.33(.03)$ & $-.54(.03)$ \\
\hline \multicolumn{7}{|c|}{$p(\sim H \mid D)$ judgements } \\
\hline $\mathrm{d}$ & $.01(.01)$ & $.06(.02)$ & $.09(.02)$ & $.03(.02)$ & $-.04(.02)$ & $.00(.03)$ \\
\hline$\neg \mathrm{d}$ & $.08(.02)$ & $.06(.02)$ & $.09(.03)$ & $.16(.05)$ & $.03(.03)$ & $.10(.05)$ \\
\hline
\end{tabular}

Note. The deviation scores in bold italics were significantly different from 0 at the .01 level.

Deviation scores were expected to vary as a function of expected direction and magnitude of deviations, defined by the difference between inverse likelihood $p(D \mid H)$ and Bayesian value $p^{*}(H \mid D)$ for the $p(H \mid D)$ judgements, and by the difference between inverse likelihood $p(D \mid \sim H)$ and Bayesian value $p^{*}(\sim H \mid D)$ for the $p(\sim H \mid D)$ judgements (where $D$ represents the conditioning datum, i.e., either $\mathrm{d}$ or $\neg \mathrm{d}$ ). To test for significant effects, the data were subjected to a 2 (conditioning datum: $D=d$, $D=\neg \mathrm{d}) \times 2$ (expected direction of deviation: overestimation, underestimation) $\times 3$ (expected magnitude of deviation: small, medium, large) Multivariate repeated measures ANOVA with $p(H \mid D)$ and $p(\sim H \mid D)$ judgements as dependent measures. A Multivariate Analysis of Variance with two dependent measures was performed rather than a repeated-measures Analysis of Variance with the type of question asked [i.e., $p(H \mid d)$ vs. $p(H \mid \neg d)$ question] as an additional factor because this experiment was not 
designed to test for the effect of the type of question asked on the posterior probability judgements observed. Table V-6 summarises the results of this analysis.

Table V-6

Summary of the 2 (conditioning datum) $\times 2$ (expected direction of deviation) $\times 3$ (expected magnitude of deviation) multivariate repeated-measure ANOVA with $p(H \mid D)$ and $p(\sim H \mid D)$ judgements as dependent measures.

\begin{tabular}{cccccc}
\hline Effect & Wilks' $\Lambda$ & $d f$ & $d f_{\mathrm{e}}$ & $F$ & Partial $\eta^{2}$ \\
\hline $\begin{array}{c}\text { Between Subjects } \\
\text { Intercept }\end{array}$ & 0.43 & 2 & 43 & $28.70^{*}$ & 0.57 \\
Within Subjects & & & & & \\
Direction (D) & 0.10 & 2 & 43 & $198.15^{*}$ & 0.90 \\
Magnitude (M) & 0.50 & 4 & 41 & $10.25^{*}$ & 0.50 \\
Conditioning datum (C) & 0.80 & 2 & 43 & $5.45^{*}$ & 0.20 \\
D $\times$ M & 0.17 & 4 & 41 & $49.89^{*}$ & 0.83 \\
$\mathrm{D} \times \mathrm{C}$ & 0.88 & 2 & 43 & 2.92 & 0.12 \\
$\mathrm{M} \times \mathrm{C}$ & 0.90 & 4 & 41 & 1.20 & 0.10 \\
$\mathrm{D} \times \mathrm{M} \times \mathrm{C}$ & 0.98 & 4 & 41 & 0.25 & 0.02 \\
\hline${ }^{*} \mathrm{p}<.05$ & & & & &
\end{tabular}

The significant main effects of the direction of deviation and the magnitude of deviation were qualified by the significant two-way (direction of deviation $\times$ magnitude of deviation) interaction effect observed [Wilks' $\Lambda=0.17$, multivariate $F(4,41)=$ 49.89]. Unexpectedly, judgements were also significantly affected by the conditioning datum [Wilks' $\Lambda=0.80$, multivariate $F(4,41)=5.45$ ] although this effect was relatively small, compared to the effect size of the expected interaction (partial $\eta^{2}=$ 0.20 vs. partial $\eta^{2}=0.83$, respectively).

The MANOVA indicates whether the factors defined have an effect, overall, on the measures taken. In order to identify the exact source of these effects, however, one needs to conduct Univariate F-tests. Table V-7 summarises the results of 2 (direction of deviation) $\times 3$ (magnitude of deviation) $\times 2$ (conditioning datum) repeated-measures univariate analyses of variance respectively for the $p(H \mid D)$ measure and the $p(\sim H \mid D)$ measures. To warrant the analysis against inflated Type I error, an $\alpha$-level of 0.02 was used for each of the two measures, to ensure that $\alpha$ for the set of $p(H \mid D)$ and $p(\sim H \mid D)$ measures does exceed the 0.05 critical value [For two dependent measures, Tabachnick and Fidell (1996, p. 402) recommended setting up $\alpha_{1}$ and $\alpha_{2}$ so that $\alpha=1-\left(1-\alpha_{1}\right)(1$ $\left.\left.-\alpha_{2}\right)\right]$. 
Table V-7

Univariate repeated-measures ANOVAs for the $p(H \mid D)$ and the $p(\sim H \mid D)$ measures

\begin{tabular}{lrrrrr}
\hline Source & $S S$ & $d f$ & $M S$ & $F$ & Partial $\eta$ \\
\hline & $p(H \mid D)$ measure & & & \\
Effects & & & & & \\
$\quad$ Direction of deviation (D) & 26.22 & 1 & 26.22 & $268.09^{*}$ & 0.86 \\
Magnitude of deviation (M) & 0.86 & 2 & 0.43 & $27.59^{*}$ & 0.39 \\
Conditioning datum (C) & 0.40 & 1 & 0.40 & $6.08^{*}$ & 0.12 \\
D $\times$ M & 4.68 & 2 & 2.34 & $106.69^{*}$ & 0.71 \\
D $\times$ C & 0.23 & 1 & 0.23 & 4.99 & 0.10 \\
M $\times$ C & 0.01 & 2 & 0.01 & 0.36 & 0.01 \\
D $\times M \times C$ & 0.02 & 2 & 0.01 & 0.39 & 0.01
\end{tabular}

Error terms

$\begin{array}{llll}\text { Direction of deviation (D) } & 4.30 & 44 & 0.10 \\ \text { Magnitude of deviation (M) } & 1.37 & 88 & 0.02 \\ \text { Conditioning datum (C) } & 2.91 & 44 & 0.07 \\ \mathrm{D} \times \mathrm{M} & 1.93 & 88 & 0.02 \\ \mathrm{D} \times \mathrm{C} & 2.02 & 44 & 0.05 \\ \mathrm{M} \times \mathrm{C} & 1.69 & 88 & 0.02 \\ \mathrm{D} \times \mathrm{M} \times \mathrm{C} & 2.15 & 88 & 0.02\end{array}$

Effects

$\begin{array}{lrrrrr}\text { Direction of deviation (D) } & 0.04 & 1 & 0.04 & 0.37 & 0.01 \\ \text { Magnitude of deviation (M) } & 0.19 & 2 & 0.09 & 4.50^{*} & 0.09 \\ \text { Conditioning datum (C) } & 0.51 & 1 & 0.51 & 10.66^{*} & 0.20 \\ \mathrm{D} \times \mathrm{M} & 0.35 & 2 & 0.17 & 8.06 * & 0.15 \\ \mathrm{D} \times \mathrm{C} & 0.20 & 1 & 0.20 & 4.57 & 0.09 \\ \mathrm{M} \times \mathrm{C} & 0.10 & 2 & 0.05 & 2.41 & 0.05 \\ \mathrm{D} \times \mathrm{M} \times \mathrm{C} & 0.01 & 2 & 0.01 & 0.27 & 0.01\end{array}$

Error terms

Direction of deviation (D)

$\begin{array}{lll}4.46 & 44 & 0.10\end{array}$

Magnitude of deviation (M)

$\begin{array}{lll}1.85 & 88 & 0.02\end{array}$

Conditioning datum (C)

$\begin{array}{lll}2.09 & 44 & 0.05\end{array}$

$\mathrm{D} \times \mathrm{M}$

$\begin{array}{lll}1.90 & 88 & 0.02\end{array}$

$\mathrm{D} \times \mathrm{C}$

$\begin{array}{lll}1.95 & 44 & 0.04\end{array}$

$\mathrm{M} \times \mathrm{C}$

$\begin{array}{lll}1.84 & 88 & 0.02\end{array}$

$\mathrm{D} \times \mathrm{M} \times \mathrm{C}$

$\begin{array}{lll}2.05 & 88 & 0.02\end{array}$

${ }^{*} p<.02$ 
Univariate $F$-tests revealed that the interaction term direction of deviation $\times$ magnitude of deviation was statistically significant for both the $p(H \mid D)$ and the $p(\sim H \mid D)$ measure.

This result is illustrated by Figure V-3. The graphs show observed deviations of mean posterior probability judgements from Bayesian values as a function of expected magnitude of deviation (small, medium, or large) and expected direction of deviation (overestimation or underestimation), collapsed across the conditioning datum ( $\mathrm{d}$ and $\neg \mathrm{d})$. The left graph shows that, for $p(H \mid D)$ judgements, observed deviations from the Bayesian values clearly varied as a function of the difference between inverse values and Bayesian values (as denoted by the bars). When overestimation was expected [i.e., when $\left.p(D \mid H)>p^{*}(H \mid D)\right], p(H \mid D)$ posterior probability judgements were higher than $p^{*}(H \mid D)$ Bayesian values. Moreover, such overestimation of normative values varied as a function of the magnitude of the deviation between inverse and Bayesian values: the higher the expected deviation, the higher the observed overestimation of Bayesian values. And, when underestimation was expected [i.e., when $p(D \mid H)<p^{*}(H \mid D)$ ], observed posterior probability judgements $p(H \mid D)$ were indeed less than the Bayesian $p^{*}(H \mid D)$ values and the extent to which Bayesian values were underestimated varied as a function of the magnitude of the difference between inverse and Bayesian values.

The right hand side graph shows that this interaction effect is less pronounced with $p(\sim H \mid D)$ judgements where deviations from Bayesian values were expected to be much lower (as denoted by the bars). 


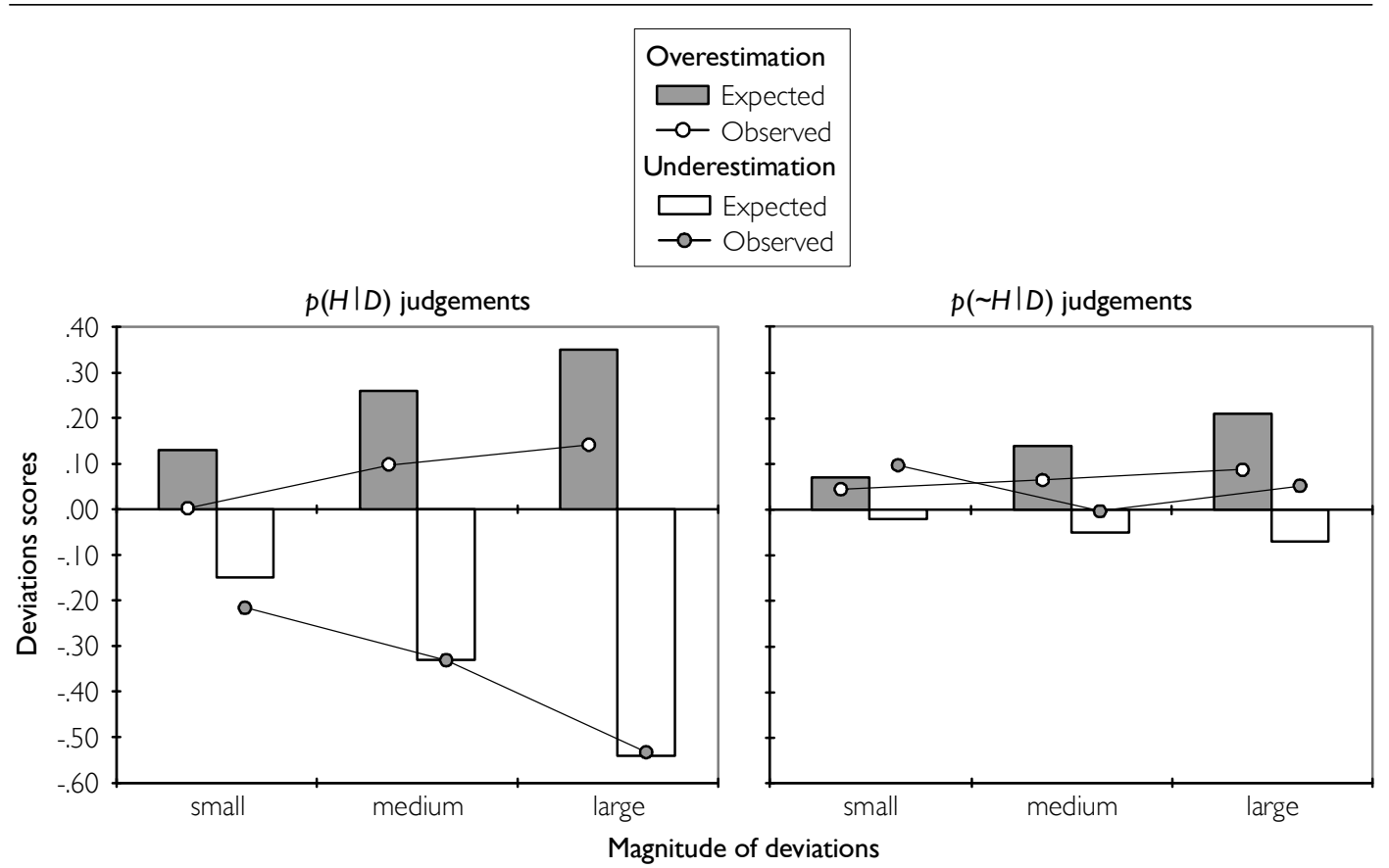

Figure V-3 - Observed deviations of $p(H \mid D)$ and $p(\sim H \mid D)$ judgements from Bayesian values as a function of expected magnitudes and expected direction of deviation.

Note. Expected values were computed by subtracting Bayesian values from corresponding inverse values in each condition.

To test for the mean differences that contributed to the interaction effect on the deviation scores for the $p(H \mid D)$ measure, planned multiple comparisons were computed. The results of these comparisons are summarised in Table V-8. The $\alpha$-level was set at .005 to warrant the analyses against an inflated Type I error. Given that these comparisons tested differences among means for a within-subject measure, separate error terms were used for each of the comparisons made (e.g., Howell, 1992; Tabachnick \& Fidell, 2001). 
Table V-8

Planned marginal comparisons for the Direction of deviation $\times$ Magnitude of deviation interaction effect for the $p(H \mid D)$ and $p(\sim H \mid D)$ measures.

\begin{tabular}{|c|c|c|c|c|c|}
\hline Contrast & SS & $d f$ & MS & $F$ & Partial $\eta^{2}$ \\
\hline \multicolumn{6}{|c|}{$p(H \mid D)$ judgements } \\
\hline (lod,lo $\neg \mathrm{d})$ vs (mod, mo $\neg \mathrm{d})$ & 0.17 & 1 & 0.17 & 5.64 & 0.11 \\
\hline Error & 1.32 & 44 & 0.03 & & \\
\hline (mod, mo $\neg$ d) vs. (sod, so $\neg$ d) & 0.82 & 1 & 0.82 & $21.72^{*}$ & 0.33 \\
\hline Error & 1.66 & 44 & 0.04 & & \\
\hline (sod, so $\neg$ d) vs. (sud, su $\neg$ d) & 4.30 & 1 & 4.30 & $100.15^{*}$ & 0.69 \\
\hline Error & 1.89 & 44 & 0.04 & & \\
\hline (sud, su $\neg$ d) vs. (mud, mu $\neg$ d) & 1.19 & 1 & 1.19 & $41.21^{*}$ & 0.48 \\
\hline Error & 1.27 & 44 & 0.03 & & \\
\hline (mud, mu $\neg$ d) vs. (lud, lu $\neg$ d) & 3.67 & 1 & 3.67 & $146.80^{*}$ & 0.77 \\
\hline Error & 1.10 & 44 & 0.03 & & \\
\hline \multicolumn{6}{|c|}{$p(\sim H \mid D)$ judgements } \\
\hline$(\mathrm{lod}, \mathrm{lo} \neg \mathrm{d})$ vs $(\mathrm{mod}, \mathrm{mo} \neg \mathrm{d})$ & 0.05 & 1 & 0.05 & 2.05 & 0.04 \\
\hline Error & 0.97 & 44 & 0.02 & & \\
\hline (mod, mo $\neg$ d) vs. (sod, so $\neg$ d) & 0.03 & 1 & 0.03 & 1.70 & 0.04 \\
\hline Error & 0.90 & 44 & 0.02 & & \\
\hline (sod, so $\neg$ d) vs. (sud, su $\neg$ d) & 0.25 & 1 & 0.25 & 3.21 & 0.07 \\
\hline Error & 3.38 & 44 & 0.08 & & \\
\hline (sud, su $\neg$ d) vs. (mud, mu $\neg$ d) & 0.91 & 1 & 0.91 & $14.93^{*}$ & 0.25 \\
\hline Error & 2.68 & 44 & 0.06 & & \\
\hline (mud, mu $\neg$ d) vs. (lud, lu $\neg$ d) & 0.28 & 1 & 0.28 & 4.50 & 0.09 \\
\hline Error & 2.71 & 44 & 0.06 & & \\
\hline
\end{tabular}

"p $<.005$

Note. Within-subject conditions were identified by their expected magnitude ('l' for large, 'm' for medium, 's' for small) and expected direction ('o' for overestimation, 'u' for underestimation) of deviation from the Bayesian norm, as well as their conditioning datum ['d' for $p(\mathrm{H} \mid \mathrm{d})$ or $p(\sim \mathrm{H} \mid \mathrm{d})$ judgements, ' $\neg \mathrm{d}$ ' for $p(H \mid \neg \mathrm{d})$ or $\mathrm{p}(\mathrm{H} \mid \neg \mathrm{d})$ judgements]. For instance, 'lod' corresponded to the condition where a large overestimation of the Bayesian value was expected, and a $p(\mathrm{H} \mid \mathrm{d})$ judgement was elicited.

These results show that, for all but one comparison, the manipulation of the difference between Bayesian and inverse values significantly affected the deviation of posterior probability judgement from Bayesian prescriptions for $p(H \mid D)$ judgements. The only non-significant difference was the difference in deviations in conditions where the expected overestimation was expected to be either large or medium. The difference 
observed was nevertheless in the expected direction $\left(\mathrm{M}_{\text {medium }}=.10, \mathrm{SE}=.03 \mathrm{vs} . \mathrm{M}_{\text {large }}=\right.$ $.14, \mathrm{SE}=.04)$.

By contrast, for $p(\sim H \mid D)$ judgements, the only significant difference was observed when a small vs. medium underestimation was expected. In this latter case, however, the expected deviations were very small (see Figure V-3). This finding thus confirms that posterior probability judgements will not significantly depart from Bayesian values when inverse values are themselves close to these Bayesian values.

As noted above (see p. 147), the MANOVA also revealed a significant main effect of the conditioning datum ( $D=\mathrm{d}$ vs. $D=\neg \mathrm{d}$ ) on deviation scores [Wilks' $\Lambda=0.80$, multivariate $F(4,41)=5.45]$. The univariate ANOVA revealed that this main effect was significant for both $p(H \mid D)$ and $p(\sim H \mid D)$ measures. It has already been suggested and observed that participants tend to be more influenced by positive answers than by negative answers (see Sherman \& Corty, 1984, Slowiaczek et al., 1992) leading to judgements based on a positive answer to be higher than those based on a negative answer. This was verified for $p\left(H \mid d_{i}\right)$ judgements $[M=.63]$ compared to $p\left(H \mid \neg d_{i}\right)$ judgements $\left[M=.58 ; F(1,44)=6.08, M S E=0.07, p=.02\right.$, partial $\left.\eta^{2}=.12\right]$. The reverse was true, however, for $p\left(\sim H \mid d_{i}\right)$ judgements $[M=.28]$ compared to $p\left(\sim \mathrm{H} \mid \neg \mathrm{d}_{i}\right)$ judgements $\left[\mathrm{M}=.34, F(1,44)=10.66, \mathrm{MSE}=0.05, p=.002\right.$, partial $\eta^{2}$ $=$. 20]. Although there is no obvious explanation available for this finding, it is an indicator of the processes that may have underlain the posterior probability judgements of participants who did not commit the inverse fallacy, as those who did rely on inverse likelihood values would have provided judgements that were independent of the conditioning datum (see design and procedure, p. 141).

\section{Deviations from the additivity principle}

The last hypothesis of this experiment stated that the observed patterns of additivity of complementary posterior probability judgements would be a function of the sum of their corresponding inverse probabilities. Additivity scores were computed for each participants by adding $p(H \mid D)$ and $p(\sim H \mid D)$ judgements for each of the 12 stimuli used. Table V-9 shows the mean additivity scores and standard errors obtained in each of the conditions. 
Table V-9

Mean additivity scores as a function of expected direction of deviation, expected magnitude of deviation and conditioning datum.

\begin{tabular}{|c|c|c|c|c|c|c|}
\hline \multirow{5}{*}{$\begin{array}{l}\text { Conditioning } \\
\text { datum }\end{array}$} & \multicolumn{6}{|c|}{ Direction } \\
\hline & \multicolumn{3}{|c|}{ Overestimation } & \multicolumn{3}{|c|}{ Underestimation } \\
\hline & \multicolumn{6}{|c|}{ Magnitude } \\
\hline & Small & Medium & Large & Small & Medium & Large \\
\hline & $M(S E)$ & $M(S E)$ & $M(S E)$ & $M(S E)$ & $M(S E)$ & $M(S E)$ \\
\hline $\mathrm{d}$ & $1.06(.03)$ & $1.20(.04)$ & $1.29(.05)$ & $0.62(.03)$ & $0.71(.04)$ & $0.60(.05)$ \\
\hline$\neg \mathrm{d}$ & $1.04(.03)$ & $1.12(.06)$ & $1.17(.08)$ & $0.72(.04)$ & $0.78(.04)$ & $0.70(.05)$ \\
\hline
\end{tabular}

Note. The deviation scores in bold italics were significantly different from 1 at the .01 level.

Additivity scores were expected to vary as a function of expected direction and magnitude of deviations of additivity, defined by the sum of inverse likelihood values $p(D \mid H)$ and $p(D \mid \sim H)$ (where $D$ represents the conditioning datum, i.e., either $\mathrm{d}$ or $\neg \mathrm{d}$ ). To test for significant effects, the data were subjected to a 2 (conditioning datum: $D=\mathrm{d}, D=\neg \mathrm{d}) \times 2$ (expected direction of deviation: subadditivity, superadditivity) $\times$ 3 (expected magnitude of deviation: small, medium, large) repeated measures Analysis of Variance (ANOVA). Table V-10 summarises the results of this analysis. F-tests revealed significant main effects of the expected direction and magnitude as well as a significant interaction between these two factors, as predicted. This result indicates that the manipulation of the magnitude and the direction (i.e., subadditivity vs. superadditivity) of the deviations from 1 of the sum $p(D \mid H)+p(D \mid \sim H)$ in turn affected the extent to which complementary posterior probability judgements deviated from 1, as well as the direction of this deviation. This interaction between expected direction and magnitude of deviation is illustrated by Figure V-4. 
Table V-10

Univariate Analyses of Variance for the additivity scores.

\begin{tabular}{|c|c|c|c|c|c|}
\hline Source & SS & $d f$ & MS & F & Partial $\eta^{2}$ \\
\hline \multicolumn{6}{|l|}{ Effects } \\
\hline Direction of deviation (D) & 28.26 & 1 & 28.26 & $88.28^{*}$ & 0.67 \\
\hline Magnitude of deviation (M) & 0.93 & 2 & 0.47 & $14.25^{*}$ & 0.24 \\
\hline Conditioning datum (C) & 0.01 & 1 & 0.01 & 0.12 & 0.00 \\
\hline $\mathrm{D} \times \mathrm{M}$ & 1.04 & 2 & 0.52 & $13.48^{*}$ & 0.23 \\
\hline $\mathrm{D} \times \mathrm{C}$ & 0.86 & 1 & 0.86 & $5.96^{*}$ & 0.12 \\
\hline $\mathrm{M} \times \mathrm{C}$ & 0.07 & 2 & 0.04 & 0.80 & 0.02 \\
\hline $\mathrm{D} \times \mathrm{M} \times \mathrm{C}$ & 0.06 & 2 & 0.03 & 0.69 & 0.02 \\
\hline \multicolumn{6}{|l|}{ Error } \\
\hline Direction of deviation (D) & 14.09 & 44 & 0.32 & & \\
\hline Magnitude of deviation (M) & 2.88 & 88 & 0.03 & & \\
\hline Conditioning datum $(\mathrm{C})$ & 2.13 & 44 & 0.05 & & \\
\hline $\mathrm{D} \times \mathrm{M}$ & 3.39 & 88 & 0.04 & & \\
\hline $\mathrm{D} \times \mathrm{C}$ & 6.36 & 44 & 0.14 & & \\
\hline $\mathrm{M} \times \mathrm{C}$ & 3.96 & 88 & 0.05 & & \\
\hline $\mathrm{D} \times \mathrm{M} \times \mathrm{C}$ & 3.96 & 88 & 0.04 & & \\
\hline
\end{tabular}




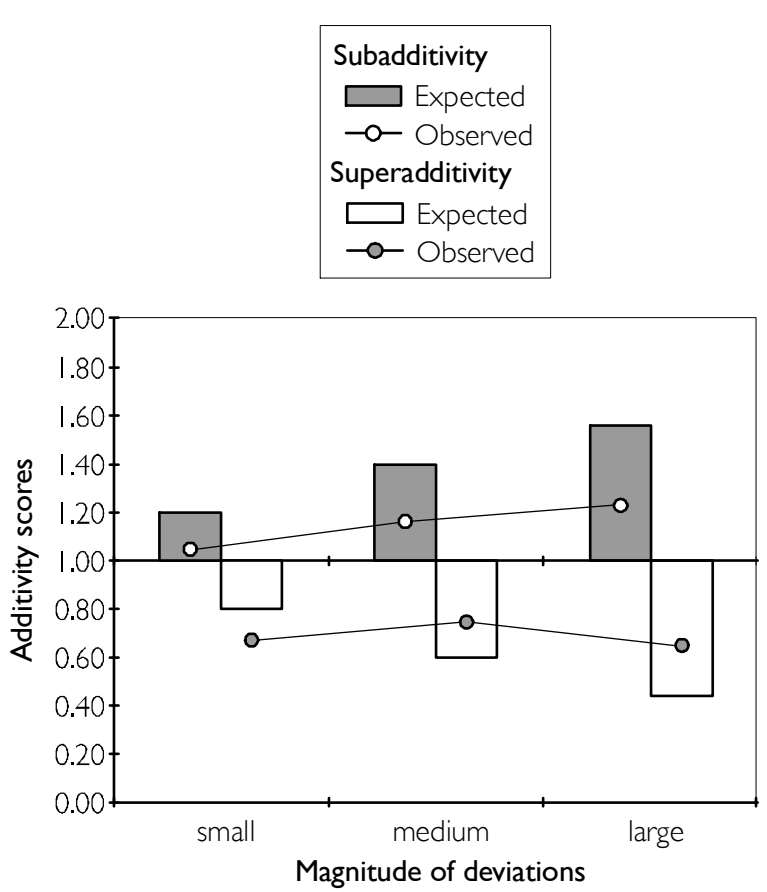

Figure V-4 - Observed summed complementary posterior probability judgements as a function of expected magnitudes of deviations and expected direction of deviation from additivity.

Note. Expected values were computed by adding inverse likelihood values in each condition.

Figure V-4 shows the mean additivity scores observed as a function of expected magnitude of deviation (small, medium, or large) and expected direction of deviation (superadditivity or subadditivity). As predicted, participants' estimates were subadditive when complementary inverse values summed to more than one and their estimates were superadditive when these probabilities summed to less than one. For either predicted subadditivity or superadditivity, the magnitude of these deviations also varied as predicted by the sum of inverse probabilities (as denoted by the bars in Figure V-4). 
Table V-11

Planned marginal comparisons for the Direction of deviation $\times$ Magnitude of deviation interaction effect for the additivity scores.

\begin{tabular}{|c|c|c|c|c|c|}
\hline Contrast & SS & $d f$ & MS & F & Partial $\eta^{2}$ \\
\hline$(\mathrm{lbd}, \mathrm{lb} \neg \mathrm{d})$ vs $(\mathrm{mbd}, \mathrm{mb} \neg \mathrm{d})$ & 0.39 & 1 & 0.39 & $11.89^{*}$ & 0.21 \\
\hline Error & 1.44 & 44 & 0.03 & & \\
\hline (mbd, mb $\neg$ d) vs. (sbd, sb $\neg \mathrm{d}$ ) & 1.19 & 1 & 1.19 & $28.81^{*}$ & 0.40 \\
\hline Error & 1.82 & 44 & 0.04 & & \\
\hline (sbd, sb $\neg$ d) vs. (spd, sp $\neg$ d) & 12.75 & 1 & 12.75 & $104.30^{*}$ & 0.70 \\
\hline Error & 5.38 & 44 & 0.12 & & \\
\hline (spd, sp $\neg$ d) vs. (mpd, mp $\neg$ d) & 0.50 & 1 & 0.50 & 6.36 & 0.13 \\
\hline Error & 3.46 & 44 & 0.08 & & \\
\hline (mpd, mp $\neg$ d) vs. (lpd, lp $\neg \mathrm{d}$ ) & 0.84 & 1 & 0.84 & $10.02^{*}$ & 0.19 \\
\hline Error & 3.68 & 44 & 0.08 & & \\
\hline
\end{tabular}

"p $<.01$

Note. Within-subject conditions were identified by their expected magnitude ('l' for large, ' $\mathrm{m}$ ' for medium, 's' for small) and expected direction ('b' for subadditivity, 'p' for superadditivity) of deviation from unity, as well as their conditioning datum ['d,' ' $\neg \mathrm{d}$ ']. For instance, 'lbd' corresponded to the condition where a large subadditivity of the sum of the complementary judgements $p(\mathrm{H} \mid \mathrm{d})$ and $p(\sim \mathrm{H} \mid \mathrm{d})$ was expected.

To test for the mean differences that contributed to the interaction effect on the additivity scores, planned multiple comparisons were computed. The results of these comparisons are summarised in Table V-11. The $\alpha$-level was set at .01 to warrant the analyses against an inflated Type I error and separate error terms were computed for each comparison. These results show that, for all but one comparison, the manipulation of the sum of $p(D \mid H)$ and $p(D \mid \sim H)$ values significantly affected the deviation of the sum of complementary posterior probability judgements from unity. The only nonsignificant difference was between the small vs. medium expected superadditivity $\left(\mathrm{M}_{\text {small }}\right.$ $=.67, \mathrm{SE}=.03$ versus $\mathrm{M}_{\text {medium }}=.75, \mathrm{SE}=.03$, respectively).

Finally, the ANOVA of additivity scores (see Table V-10, p. 154) also revealed a significant two-way (expected direction of deviation $\times$ conditioning datum) interaction $\left[F(1,44)=5.96, \mathrm{MSE}=0.14\right.$, partial $\left.\eta^{2}=0.12\right]$. Further contrast analyses revealed that this effect was due to a significant effect of the conditioning datum factor when superadditivity was expected (i.e., when the sum of complementary judgements was expected to be less than 1). The sum of $p(\mathrm{H} \mid \mathrm{d})$ and $p(\sim \mathrm{H} \mid \mathrm{d})$ judgements was lower than the sum of $p(H \mid \neg d)$ and $p(\sim H \mid \neg d)[M=.65, S E=.03$ vs. $M=.73$, SE = .04; 
$F(1,44)=5.98, \mathrm{MSE}=0.25 ; \alpha$-level $=0.025$ to protect the analysis against inflated Type I errors].

\section{Discussion of Experiment 3}

Consistent with previous research (e.g., Bar-Hillel, 1980; Eddy, 1982; Hamm, 1993, Wolfe, 1995) and in support of the first hypothesis of this experiment, the results obtained revealed that roughly half of the sample equated the posterior and inverse probabilities on over $80 \%$ of the judgement trials. This is a compelling finding because, in past studies demonstrating the inverse fallacy, typically only one judgement per participant was solicited. By contrast, it was demonstrated that a sizeable proportion of judges consistently used an inverse fallacy algorithm over a set of judgement tasks that varied in terms of both the target and conditioning event of the question asked and the values of the available probability information. It was also demonstrated that the direction and magnitude of deviations of participants' judgements from both Bayesian posterior probabilities and the additivity principle were successfully predicted by the deviation between the inverse and Bayesian posterior probabilities, thus supporting the second and third hypotheses, respectively. Furthermore, deviations from normative benchmarks could not be strictly accounted for by the base rate fallacy because base rates for the two relevant categories were always equal. Indeed, had participants only ignored base-rate information, their judgements would not have deviated from the Bayesian values.

The proportion of participants who consistently committed the inverse fallacy was higher in this experiment than is usually reported. For instance, whereas in the present experiment, $57 \%$ (613 out of 1080) of the judgements elicited could be classified as 'inverse likelihood judgements,' by contrast, Gigerenzer and Hoffrage (1995) observed that about 23\% (99 out of 433) of the judgements elicited in a standard Textbook problems could be classified as such. The percentage of inverse likelihood judgements in this experiment was computed by evaluating the number of inverse judgements observed out of the 1080 (45 participants $\times 24$ judgements) judgements collected. The percentage of inverse likelihood judgements in Gigerenzer and Hoffrage's (1995) experiment was computed from the proportions of 'Fisherian' and 'adjusted Fisherian' algorithms observed by the authors (see Gigerenzer \& Hoffrage, 1995, Table 3, p. 695) in Textbook problems. The proportions observed by Gigerenzer and Hoffrage (1995) and those observed in the present experiment were treated as independent binomial 
samples. Following Agresti (1996, pp. 20-21), the estimated standard error of the difference in proportion of inverse judgements observed was defined as

$$
\hat{\sigma}\left(p_{1}-p_{2}\right)=\sqrt{\frac{p_{1}\left(1-p_{1}\right)}{N_{1}}+\frac{p_{2}\left(1-p_{2}\right)}{N_{2}}} .
$$

The proportion of inverse judgements observed by Gigerenzer and Hoffrage (1995) was significantly lower than that observed in the present experiment $[S E=0.03, z=13.46$, $p<.01]$

There are several possible explanations for this difference: the tasks used by Gigerenzer and Hoffrage (1995) differed both in content and context from the present task; that is, not only did the cover story differ, but also all but one of the tasks used by Gigerenzer and Hoffrage (1995) referred to natural social categories. Moreover, the authors used a more restrictive criterion for their classification (see p. 144). Yet another difference was structural, namely, the participants in Experiment 3 were asked to estimate both $p(H \mid D)$ and $p(\sim H \mid D)$ for each stimulus presented, whereas textbook problems generally only elicit $p(H \mid D)$ judgements.

It is possible that the presence of these two complementary questions in this experiment somehow induced participants to assume that the $p(D \mid H)$ information was only relevant for the $p(H \mid D)$ question and that the $p(D \mid \sim H)$ information was only relevant for the $p(\sim H \mid D)$ question, rather than considering that both $p(D \mid H)$ and $p(D \mid \sim H)$ were relevant for the $p(H \mid D)$ as well as the $p(\sim H \mid D)$ question. In contrast, when the same information is presented but the task only consists in evaluating $p(H \mid D)$, some participants may assume that both $p(D \mid H)$ and $p(D \mid \sim H)$ are relevant for the judgement at hand. This inference would therefore discourage them from committing the inverse fallacy [i.e., equating $p(H \mid D)$ with $p(D \mid H)$ ] and instead motivate them to integrate both the value of $p(D \mid H)$ and $p(D \mid \sim H)$ in their final judgement. Figure V-5 illustrates this argument. A detailed explanation of the origin of these differences in relevance attribution will be developed in Chapter VII. 

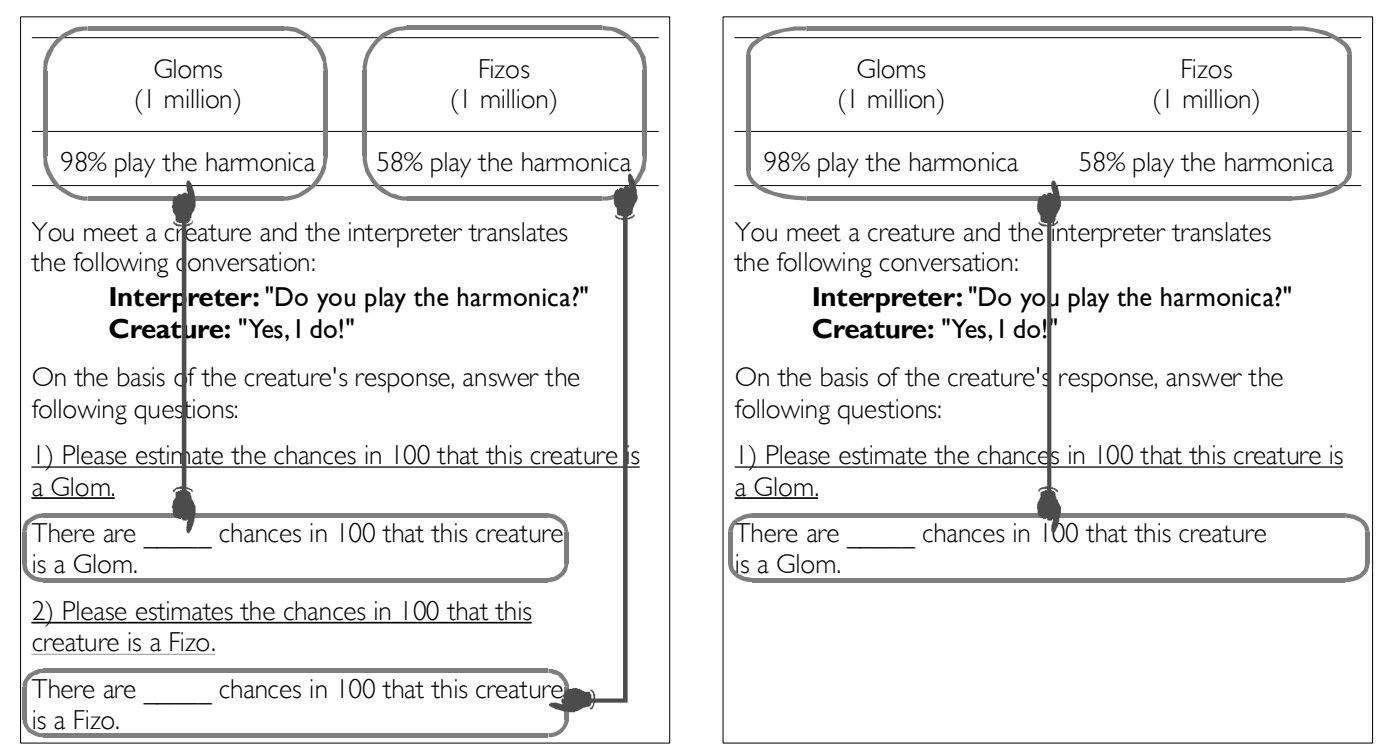

Figure V-5 - Hypothesized use of probability information, as a function of the questions asked (single vs. complementary)

This could explain why the proportion of inverse fallacy judgements was higher in the present experiment. This also suggests that if participants were either asked to estimate $p(H \mid D)$ or $p(\sim H \mid D)$ but not both, the overall proportion of inverse fallacy judgements elicited should decrease significantly. In support of the hypothesis that the presence of a unique task goal will induce participants to combine $p(D \mid H)$ and $p(D \mid \sim H)$ was the fact that the proportion of Bayesian judgements reported by Gigerenzer and Hoffrage (1995) was significantly higher than the proportion of Bayesian judgements reported here $[16 \%$ vs. $9 \%$, respectively, $S E=0.02, z=-7.34, p<.01$; Agresti, 1996; see p. 158], which suggests that a higher proportion of Bayesian judgements should be expected when participants are asked to estimate $p(H \mid D)$ or $p(\sim H \mid D)$ but not both. A second experiment was designed to test these predictions, while controlling for the effect of task content and context.

\section{EXPERIMENT 4}

This experiment was designed to test the hypothesis that the proportion of individuals committing the inverse fallacy would decrease when the task elicited only one of two complementary probability judgements. The same probability problems used in Experiment 3 were presented to participants. In the present experiment, however, participants were asked to estimate either $p(H \mid D)$ or $p(\sim H \mid D)$, but not both. The first hypothesis tested in this experiment was that the presence of a unique goal statement would lead to a decrease in the proportion of participants who committed the inverse 
fallacy, compared to the proportion of participants who committed the inverse fallacy when asked to judge $p(H \mid D)$ and $p(\sim H \mid D)$ together in sequence (Experiment 3). Given that the presence of a unique goal statement was expected to lead some participants to aggregate the inverse likelihoods $p(D \mid H)$ and $p(D \mid \sim H)$, it was also expected that the proportion of Bayesian estimates elicited from this task, while remaining low, would still be higher than that observed when participants were prompted to judge $p(H \mid D)$ and $p(\sim H \mid D)$ together in sequence.

\section{Method}

\section{Participants}

Fifty-five University of Hertfordshire undergraduates (32 women, 19 men, and 4 unspecified, mean age $=30.3$ years, age range $=18-65)$ participated in the experiment for course credit. They were tested individually and were randomly assigned to one of two experimental groups.

\section{Materials and Procedure}

Participants were provided with a 13-page questionnaire. A first group of participants, the 'Glom' or $p(H \mid D)$ group, were asked to make $p(H \mid D)$ judgements whereas a second group, the 'Fizo' or $p(\sim H \mid D)$ group, were asked to make $p(\sim H \mid D)$ judgements. The first page replicated the presentation of the task used in Experiment 3 except that participants in the Glom group were told "Each time you meet one of the invisible creatures, you want to know whether it is a Glom" and participants in the Fizo group were told "Each time you meet one of the invisible creatures, you want to know whether it is a Fizo." Participants then were provided with an example and were either told that they would be asked to estimate the likelihood that the creature encountered was a Glom on the basis of its answer (Glom group) or they were told that they would be asked to estimate the likelihood that the creature was a Fizo, on the basis of its answer (Fizo group). Participants "met" 12 creatures one by one on the subsequent pages of the questionnaires. For each of these 12 encounters, the creature was asked about a different feature and participants were provided with a reminder of the number of Gloms and Fizos on the planet as well as the percentages of each type of creature that possessed the feature. The stimuli used were the same as those summarised in Table V-1 and the layout was identical to that presented in Figure V-1 (see p. 140) except that participants 
were either asked for the posterior probability that the creature encountered was a Glom $[p(H \mid D)]$ or they were asked for the posterior probability that it was a Fizo $[p(\sim H \mid D)]$.

\section{Stimuli and design}

For each of the two experimental groups, each of the 12 encounters represented a unique stimulus condition in a 2 (conditioning datum: $D=\mathrm{d}, D=\neg \mathrm{d}) \times 2$ (expected direction of deviation: overestimation, underestimation) $\times 3$ (expected magnitude of deviation: small, medium, large) fully-crossed mixed design with a unique dependent variable measured for each stimulus. Each of the 12 stimuli used required one estimate, and each single estimate corresponded to distinct Bayesian and inverse values (as shown in Table V-2, p. 143). As for the first experiment, the creature's response was not expected to affect participants' estimates given the diagnostic probabilities presented. The stimuli were presented in one of two randomised orders (Order $1=\left\{f_{3}, f_{5}, f_{9}, f_{2}, f_{4}\right.$, $\left.\mathrm{f}_{6}, \mathrm{f}_{5}, \mathrm{f}_{10}, \mathrm{f}_{8}, \mathrm{f}_{12}, \mathrm{f}_{1}, \mathrm{f}_{7}\right\}$; Order $2=\left\{\mathrm{f}_{7}, \mathrm{f}_{1}, \mathrm{f}_{12}, \mathrm{f}_{8}, \mathrm{f}_{10}, \mathrm{f}_{5}, \mathrm{f}_{6}, \mathrm{f}_{4}, \mathrm{f}_{2}, \mathrm{f}_{9}, \mathrm{f}_{5}, \mathrm{f}_{3}\right\}$ where $\mathrm{f}_{i}$ corresponds to the stimulus presenting feature $i$, as described in Table V-1, p. 141).

\section{Results}

\section{Inverse fallacy test}

In order to establish whether participants consistently committed the inverse fallacy in this experiment, each estimate was compared to the value of its corresponding inverse probability. An estimate was classified as inverse if it was equal to its corresponding inverse value, respectively, within a margin of error of \pm .02 . The first hypothesis of this experiment was that the number of judgements matching their corresponding inverse value per participant would decrease when the task goal was either to estimate $p(H \mid D)$ or $p(\sim H \mid D)$ but not both. A direct test of the first hypothesis is provided by the comparison of the distribution of the number of inverse matches in Experiment 3 and Experiment 4, respectively. Figure V-6 shows these distributions, collapsed across judgement type [i.e., $p(H \mid D)$ vs. $p(\sim H \mid D)]$. 


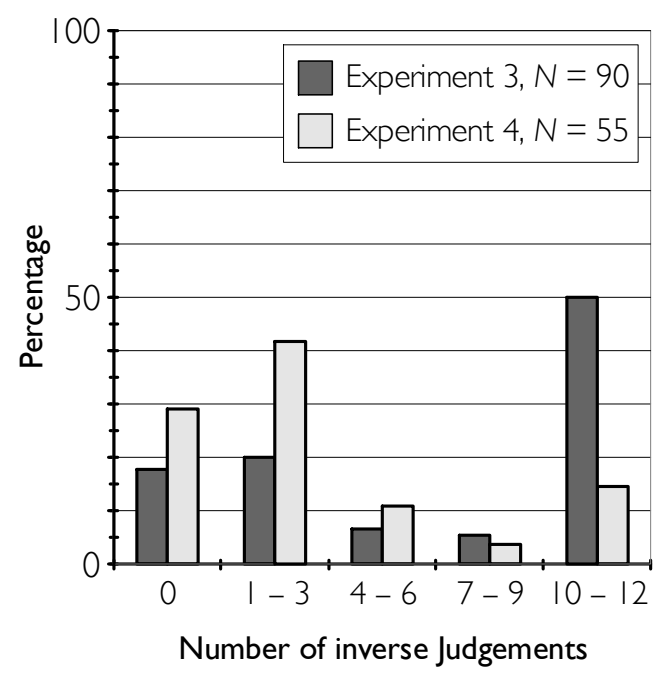

Figure V-6 - Distribution of the number of judgements matching their corresponding inverse values in Experiments 3 and 4, respectively.

Note. $\mathrm{N}$ refers to the total number of series of either $p(H \mid D)$ or $p(\sim H \mid D)$ judgements elicited.

Figure V-6 reveals that the proportion of series of twelve judgements matching at least ten out of twelve corresponding inverse values (i.e., matching an inverse value over $80 \%$ of the time) was greatly reduced in Experiment 4. To evaluate whether the distribution of inverse matches varied as a function of the experimental manipulation [i.e., asking for $p(H \mid D)$ and $p(\sim H \mid D)$ judgements in sequence (Experiment 3 ) vs. asking for either $p(H \mid D)$ or $p(\sim H \mid D)$ in isolation (Experiment 4)], the data were subjected to a Mann-Whitney $U$ test. The test revealed that this experimental manipulation indeed significantly affected the distribution of inverse judgements observed $\left(\Sigma R_{3}=7495, N_{3}=90, \Sigma R_{4}=3090, N_{4}=55, U=1550, z=-3.94\right.$, where $\Sigma R_{3}$ and $\Sigma R_{4}$ correspond to the sum of ranks in Experiment 3 and 4, respectively). The lower sum of ranks observed in Experiment 4 confirmed that there were significantly fewer individuals consistently committing the inverse fallacy over the set of 12 judgement trials. This result thus validated the first hypothesis of Experiment 4. In order words, it confirmed that the absence of contiguous complementary goal statements was sufficient to reduce the proportion of participants who consistently committed the inverse fallacy.

\section{Bayesian test}

The presence of a unique goal statement was expected to induce some participants to aggregate the inverse likelihoods $p(D \mid H)$ and $p(D \mid \sim H)$. In support of this hypothesis was the fact that the proportion of Bayesian judgements reported by Gigerenzer and 
Hoffrage (1995) was significantly higher than the proportion of Bayesian judgements reported in Experiment 3, although both proportions remained low (16\% vs. 9\%, see p. 159). Therefore, when participants are asked to estimate $p(H \mid D)$ or $p(\sim H \mid D)$ but not both, it was hypothesized that the proportion of consistent Bayesian judgement would increase; that is, more participants were expected to produce 10 or more Bayesian answers out of the 12 judgements required. Given that the rate of Bayesian judgements observed is generally low, anyhow, the majority of participants were still expected to provide non-Bayesian answers.

To test that the presence of a unique goal statement was not enough to significantly affect the proportion of participants who systematically evaluated $p(H \mid D)$ with the correct ratio of $p(D \mid H)$ and $p(D \mid \sim H)$, the distribution of the number of Bayesian matches in Experiments 3 and 4 were compared. Figure V-7 shows these distributions, collapsed across judgements type [i.e., $p(H \mid D)$ vs. $p(\sim H \mid D)]$.

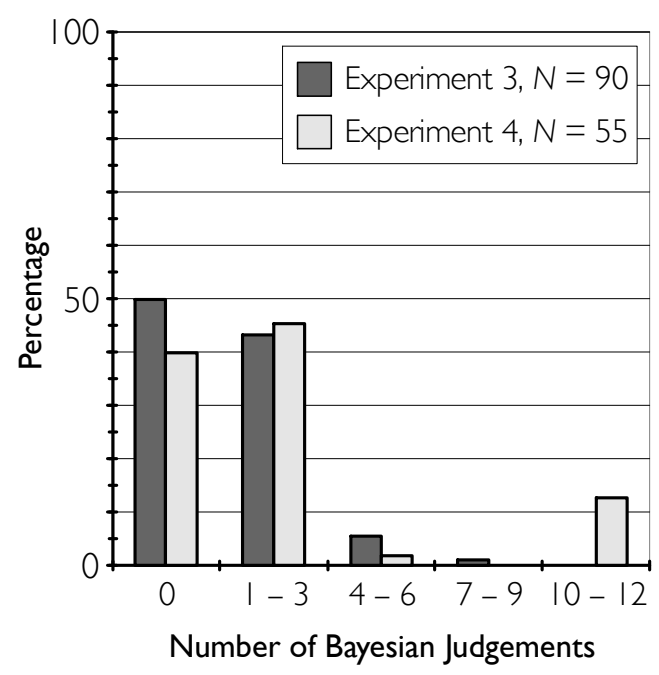

Figure V-7 - Distribution of the number of judgements matching their corresponding Bayesian values in Experiments 3 and 4, respectively.

Note. $N$ refers to the total number of series of either $p(H \mid D)$ or $p(\sim H \mid D)$ judgements elicited.

As expected, the distributions of the number of Bayesian matches in the series of twelve judgements were very similar in both experiments. That is, the majority of participants produced at most 3 judgements matching the Bayesian value. The main distinction between the distribution of the number of Bayesian matches between Experiment 3 and Experiment 4, however, was the higher proportion of participants who consistently provided Bayesian judgements. To evaluate whether the distribution of Bayesian matches varied as a function of the experimental manipulation [i.e., asking for $p(H \mid D)$ and $p(\sim H \mid D)$ judgements in sequence (Experiment 3$)$ vs. asking for either 
$p(H \mid D)$ or $p(\sim H \mid D)$ in isolation (Experiment 4$)]$, the data were subjected to a MannWhitney $U$ test. The test revealed that this experimental manipulation did not significantly affect the distribution of Bayesian judgements observed $\left(\Sigma R_{3}=6221, N_{3}=\right.$ 90, $\left.\Sigma R_{4}=4364, N_{4}=55, U=2126, z=-1.58\right)$. This result confirms that the manipulation of the task goals (i.e., single vs. complementary) was not sufficient to consistently elicit Bayesian judgements from a majority of participants. The fact that the sum of ranks in Experiment 4 was higher than what was observed in Experiment 3, however, confirms that single-goal experiments tend to elicit higher proportions of Bayesian judgements from participants.

In fact, the total proportion of Bayesian judgements observed significantly increased when participants were only asked to estimate one posterior probability. This is consistent with the fact that, whereas no series matched at least 10 out of 12 Bayesian values in Experiment 3, 13\% (7 out of 55) series did so in Experiment 4. Indeed, 19\% (128 out of 660) of the judgements observed in Experiment 4 were Bayesian, a significant increase compared to the $9 \%$ (100 out of 1080) of Bayesian judgements observed in Experiment 3 ( $\mathrm{SE}=0.02, z=5.71, p<.01$; Agresti, 1996; see p. 158).

\section{Discussion of Experiment 4}

This experiment was designed to test the prediction that the inverse fallacy would be less prevalent when individuals are either asked to estimate $p(H \mid D)$ or $p(\sim H \mid D)$ but not both, compared to when they are asked to judge $p(H \mid D)$ and $p(\sim H \mid D)$ together in sequence. The comparison of the proportion of inverse matches in a series of 12 judgements in Experiment 3 (where contiguous complementary judgements were elicited) and in Experiment 4 (where unique judgements were elicited) revealed that this seemingly inconsequential change in the design significantly affected the extent to which individuals were prone to commit the inverse fallacy.

The second hypothesis tested was that the presence of a unique goal statement would not be sufficient to elicit Bayesian judgements from the majority of participants, although the proportion of individuals consistently providing Bayesian judgements was expected to increase significantly. Again, this hypothesis was supported by the data. Indeed, the distribution of the number of judgements matching their corresponding Bayesian value in a series of 12 judgements did not significantly differ when participants were asked to provide either $p(H \mid D)$ or $p(\sim H \mid D)$ judgements (Experiment 4 ) compared with when participants were asked to provide both $p(H \mid D)$ and $p(\sim H \mid D)$ judgements 
(Experiment 3), thereby showing that the manipulation was not sufficient to systematically elicit Bayesian judgements from participants. Yet, the presence of a unique goal did lead to a significant increase in the proportion of participants whose series of 12 judgements matched the Bayesian on at least 10 occasions.

Altogether, these results demonstrate that the presence of a unique goal statement indeed led some participants to integrate both $p(D \mid H)$ and $p(D \mid \sim H)$ likelihoods in their judgements, sometimes even in a normative manner. This indicates that the judgement methods applied to solve the tasks are determined by seemingly inconsequential changes (here, the presence or absence of two complementary goal statements). At present, there is no algorithmic account that can satisfactorily explain the origin of these findings. The causality account (Tversky \& Kahneman, 1980; see chap. III, p. 58) indeed cannot be applied here since in both experiments the use of unnatural categories prevented any causal interpretations to be drawn. An extension of the relevance account (Bar-Hillel, 1980; see chap. III, p. 59) may be applicable: the presence of a unique goal statement was expected to induce some participants to aggregate both $p(D \mid H)$ and $p(D \mid \sim H)$ information. This prediction was based on the conjecture that, in such cases, these participants would infer that both pieces of information were relevant for the judgement at hand. By contrast, only $p(D \mid H)$ would be assumed to be relevant for making $p(H \mid D)$ judgement when the task's goal was to estimate both $p(H \mid D)$ and $p(\sim H \mid D)$. But this conjecture neither explains what cognitive processes determined such different attributions of relevance, nor why some participants still committed the inverse fallacy in the second experiment. Finally, Macchi's (1995; see chap. III, pp. 60) partitive account cannot explain the results observed either: the structure of the information required for the judgements or the type of questions asked were identical in both experiments. Consequently, the effects observed could not have been predicted from the partitive account. By contrast, as it will be shown later in this thesis, these findings can be explained by Sample Space Representation theory, the new theoretical account that will be developed in chapter VII.

\section{CONCLUSIONS}

This chapter pursued the process-focused investigation of posterior probability judgements, by studying such judgements within the Textbook-problem paradigm where 
all the components of Bayes's theorem for binary hypotheses [namely, $p(H), p(\sim H)$, $p(D \mid H)$, and $p(D \mid \sim H)]$ are presented in a numerical form. The first experiment was aimed at distinguishing between the inverse fallacy account and the base-rate neglect account for describing the judgement methods underlying the posterior probability judgements observed in Textbook problems. For this purpose, base rates for complementary hypotheses were set at .50 so that if participants were only neglecting base-rates, their judgements could be expected to be in line with Bayesian prescriptions. By contrast, if participants were committing the inverse fallacy, their judgements were expected to deviate from Bayesian prescriptions. It was demonstrated that roughly half of the sample of participants committed the inverse fallacy on over $80 \%$ of 12 judgement trials that varied both in terms of the values of the probability information available and the posterior probability judgement elicited.

This result in turn formed the basis for the demonstration of the importance of a precise knowledge of the methods underlying observed judgement outcomes for understanding as well as predicting deviations from normative benchmarks such as Bayes's theorem or the additivity principle. In particular, it was demonstrated that the direction and magnitude of deviations of participants' judgements from Bayesian posterior probabilities were determined by the deviation between the inverse likelihoods provided in the tasks and their corresponding Bayesian posterior probabilities. With regards to the additivity issue, it was also possible to predict whether binary complementary posterior probability judgements would be subadditive or superadditive, as a function of the sum of their corresponding inverse likelihoods.

A comparison of the proportions of inverse fallacy and Bayesian judgements generally observed in Textbook problems (based on the data provided by Gigerenzer \& Hoffrage, 1995) revealed both that the proportion of inverse judgements observed in Experiment 3 was higher than what is usually observed and that the proportion of Bayesian judgements observed was lower than what is usually observed. It was proposed that this difference was due to the fact that participants in Experiment 3 were asked to make complementary judgements together in sequence, whereas standard Textbook problems usually only ask for one posterior probability judgement. Accordingly, it was hypothesized that the presence of a unique goal statement would induce some participants to aggregate the values of $p(D \mid H)$ and $p(D \mid \sim H)$ in order to evaluate the posterior probability requested. Consequently, the presence of a unique goal statement was expected to lead to both a decrease in the proportion of participants systematically 
committing the inverse fallacy and an increase in the proportion of participants who systematically produced Bayesian judgements.

Results from Experiment 4 confirmed the inverse fallacy was less prevalent when individuals are either asked to estimate $p(H \mid D)$ or $p(\sim H \mid D)$ but not both, rather than when they are asked to judge $p(H \mid D)$ and $p(\sim H \mid D)$ together in sequence. Moreover, although the vast majority of participants' judgements did not match their corresponding Bayesian values, the proportion of participants who systematically provided Bayesian judgements did significantly increase when the task only consisted in evaluating either $p(H \mid D)$ or $p(\sim H \mid D)$ but not both.

There are both methodological and theoretical implications of these findings. From a methodological standpoint, the collection of multiple estimates from each participant in the present research protected against unwarranted conclusions resulting from the confusion between the outcome of a test and the computational process leading to this outcome. Girotto and Gonzalez (2001), for instance, showed that some of Cosmides and Tooby's (1996) observations were erroneously classified as accurate judgements by these authors on the basis of parity between participants' posterior probability estimates and the numerical values computed by Bayes's theorem. In the present study, in contrast, the collection of multiple estimates prevented the mistaken conclusion, in Experiment 3, that judgements of $p(\sim H \mid D)$ were normative when they showed little deviation from the Bayesian norm (see Figure V-3, p. 150). A more likely explanation, supported by the results observed for the $p(H \mid D)$ judgements, is that $p(\sim H \mid D)$ judgements were based on the inverse fallacy whose outputs happened to be similar to those arising from Bayes's theorem. Similarly, in Experiment 4, whereas the majority of series of twelve judgements matched either an inverse value or a Bayesian value on up to 3 occasions, very few individuals provided series of judgements that inconsistently matched either of these values, allowing us to distinguish between participants whose judgement methods might have led to a judgement output that 'accidentally' coincided with a Bayesian or an inverse value from those participants who systematically applied either a Bayesian or an inverse method to produce their judgement outputs.

The fact that the inverse fallacy is associated with nonadditive posterior probability judgements, as demonstrated in Experiment 3, also has significant methodological implications. For instance, Slowiaczek et al. (1992, Experiment 1A) assumed that their participants' judgements were additive and combined estimates of $p(H \mid D)$ with estimates of $p(\sim H \mid D)$ subtracted from 1 . This recoding procedure may have induced a 
bias in their results. For instance, when $p(D \mid H)=.5, p(D \mid \sim H)=.10$ and $D$ is present (a stimulus that was also used by Slowiaczek et al., 1992), $p(H \mid D)=.83$ and $p(\sim H \mid D)$ $=.17$. A judge applying the inverse method, would estimate $p(H \mid D)$ and $p(\sim H \mid D)$ to be .50 and .10 , respectively. Recoding $p(\sim H \mid D)$ as a $.90(1-.10)$ estimate of $p(H \mid D)$ results in a near-normative answer, whereas the judge applying the inverse method would have been more likely to estimate $p(H \mid D)$ to be .50 (the value of $p(D \mid H)$ ), thus underestimating the normative value by more than a $30 \%$ difference.

This study also has important theoretical implications as it extended the study of additivity to conditional probability judgements. Specifically, it was demonstrated that the pattern of subadditivity and superadditivity observed for participants' judgements could be predicted from the sum of the inverse probabilities. Rottenstreich and Tversky (1997) specified that the binary complementarity predicted by Support theory (Tversky $\&$ Koehler, 1994) applies to cases where the alternative hypothesis is explicitly described as such. This precision implies that, in the present study, support theory would only predict additivity for measures of $p(H \mid D)$ and $p(\neg H \mid D)$ where $\neg H$ corresponds to the explicit negation (e.g., "not Glom") as opposed to $\sim H$, which corresponds to an implicit negation (e.g., "Fizo"). In other words, Support theory predicts that, in Experiment 3, judgements would have been additive if individuals had been asked to estimate 'the probability that the creature was a Glom given its response' on the one hand and 'the probability that the creature was not a Glom given its response,' on the other hand. Rottenstreich and Tversky (1997) nevertheless argued that when the negation of the hypothesis unambiguously defines the alternative event, judgements are likely to be additive. Still, although it was made clear to participants in the present context that the creature could only be a Glom or a Fizo (and obviously, not both), the results strongly indicate that judgements were not additive. Future research might examine the effect of implicitly versus explicitly negating the alternative hypothesis on the additivity of posterior probability judgements in cases of binary complementarity.

Finally, the results observed in Experiment 4 call for the development of an algorithmic account of the reasoning processes underpinning the application of the judgement methods which led to the outputs observed. It was argued that existing accounts for the cognitive processes at work in Textbook problems (Bar-Hillel, 1980; Tversky \& Kahneman, 1980; Macchi, 1995, 2000) cannot satisfactorily explain why the presence or absence of complementary goal statements affected the judgement methods applied by participants. Sample Space Representation theory, a new general algorithmic 
account developed in chapter VII, will provide such an explanation. Meanwhile, the next chapter will conclude the presentation of the empirical work addressing computational issues highlighted by the application of a process-focused approach to the study of posterior probability judgements within the latest paradigm of research on posterior probability judgements, namely the Set-based paradigm.

Sk 
T $n$ this final empirical chapter, the process-focused study of posterior probability judgements is extended to the latest paradigm of research developed, which I called the Set-based judgement paradigm. This paradigm can again be distinguished from the two earlier paradigms reviewed (namely, the Social-judgement and the Textbook-problem paradigms) not only with regards to the type of tasks it draws on and the processes underlying the judgement outcomes observed with these tasks, but also with regards to the research issues it addresses. Set-based judgement tasks, like Textbook problems, present numerical information for all the components of Bayes's theorem [i.e., $p(H), p(\sim H), p(D \mid H)$, and $p(D \mid \sim H)$ ]. Yet, in Set-based tasks, focus is drawn on the format and the structure of this numerical information (e.g., Cosmides \& Tooby, 1996; Gigerenzer \& Hoffrage, 1995; Girotto \& Gonzalez, 2001; Macchi, 2000), as well as the type of the goal statement that will elicit the judgement (Girotto \& Gonzalez, 2001).

In sharp contrast with results observed with the Social-judgement and the Textbook-problem paradigms, judgement outcomes in Set-based tasks are characterised by high proportions of outcomes coinciding with their corresponding Bayesian criterion. Accordingly, and in contrast to the two previous paradigms identified, the core issues addressed within the Set-based paradigm are no longer related to the identification of the factors that would reduce observed deviations from normative prescriptions. Instead, researchers working within the Set-based paradigm have debated the origin of the normative judgements observed (Evans et al., 2000; Gigerenzer \& Hoffrage, 1995; Girotto \& Gonzalez, 2001, 2002; Hoffrage, et al., 2002; Macchi, 2000).

The first issue of contention identified by the review of the research carried out within the Set-based paradigm (see chap. III, p. 63 onwards) was related to the role of the format of the information presented in fully inclusive Set-based tasks. Gigerenzer and Hoffrage (1995; see also Hoffrage et al., 2002) had originally proposed that the presentation of the probabilistic information in a natural frequency format was both necessary and sufficient to elicit Bayesian outcomes. Natural frequency formats are 
defined as a frequency format arising from the natural sampling of events, whereby absolute frequencies of events are recorded sequentially while those events are observed. Gigerenzer and Hoffrage (1995) also distinguished natural frequency formats from normalised frequency formats, whether absolute or relativised (see chap. III, p. 63). New evidence shows that whereas the natural frequency format may be sufficient to elicit Bayesian judgements, it is by no means necessary. Indeed, the use of alternative formats such as the partitioned chance format (Girotto \& Gonzalez, 2001) or even that of a relativised frequency format (Macchi, 2000) have proven as successful in eliciting Bayesian outcomes as natural frequency formats.

Beyond the role of the information format, the critical aspect for eliciting Bayesian outcomes was identified as being the type of sets defined by the Set-based tasks. In particular, multiple and fully inclusive Set-based tasks were distinguished. Whereas multiple Set-based tasks define several reference sets and subsets, fully inclusive Setbased tasks were characterised as defining subsets that are all explicitly included in a unique reference set $\mathscr{S}$, and whose goal statement also refers to a set explicitly included in $\mathscr{S}$ as defined by the task's statements. It was observed that only the latter type of tasks elicit judgement outcomes that consistently coincide, for the most part, with their corresponding Bayesian outcome. By contrast, the high proportions of Bayesian outcomes observed with multiple Set-based tasks (e.g., see Cosmides \& Tooby, 1996) were found to be an artefact created by the numerical values used in those tasks (Evans et al., 2000; Girotto \& Gonzalez, 2001; Macchi \& Mosconi, 1998).

These issues are characteristic of an outcome-focused perspective, which endeavours to identify the conditions that mediate Bayesian performance. By contrast, the processfocused approach followed in this thesis sets a different research agenda, whose primary aim is to provide a systematic description of the judgement methods underlying observed outcomes. This in turn allows fostering of the subsequent development of a sound theoretical account of the cognitive processes that underpin the application of those methods. The process-focused approach led to the identification of yet another line of argument that has not received much attention. In most research work within the Setbased paradigm, researchers indeed seem to endorse the conclusion, either explicitly or implicitly, that Bayesian outputs necessarily point towards underlying Bayesian reasoning processes. In other words, from the observation that Set-based tasks elicited high 
proportions of judgement outcomes coinciding with the Bayesian criterion, authors concluded that those tasks facilitated Bayesian reasoning.

Yet, from a process-focused perspective, the simple matching of judgement outputs with Bayesian values is insufficient for determining whether people actually engage in Bayesian reasoning. Gigerenzer and Hoffrage (1995) already stressed the importance of establishing the final classification of judgements (e.g., as Bayesian or not) on the measure of both outcome value and the algorithm by which individuals arrived at this value. As a result, only Bayesian outcomes based on a Bayesian algorithm were classified as Bayesian by these authors. In other words, Gigerenzer and Hoffrage (1995) took the correct application of a Bayesian algorithm as evidence of Bayesian reasoning.

Referring to the specific case of Set-based problems, Howson and Urbach (1993) contended that equating Bayesian judgement with Bayesian reasoning was fallacious and argued that those problems only demonstrated participants' arithmetical abilities. Indeed, a standard "two-step" question (Girotto \& Gonzalez, 2001) will ask participants to calculate the number of elements in set $D \cap H$ out of the number of elements in set D. For example, the two-step question 'How many patients presenting the symptom actually have the disease? __ out of __ ' asks for the number of elements in the set of patients who have the symptom and the disease $(D \cap H)$ out of the number of elements in the set of patients who have the symptom (D). Paradoxically, although the base-rate information is irrelevant to the evaluation of this ratio, the same evaluation of this ratio of numbers when no base-rate information is presented cannot be taken as evidence of the application of a Bayesian algorithm, let alone evidence for Bayesian reasoning (see chap. III, p. 67 onwards).

So, if the correct evaluation of the proportion of elements in the set $D \cap H$, given the reduced reference set $D$ cannot be taken as evidence of Bayesian reasoning in Setbased tasks, how can we assess whether individuals demonstrate Bayesian reasoning? By contrasting algorithmic processes as the mechanical application of a set of rules and reasoning as a thoughtful inferential process underlying judgements, it was possible to define Bayesian reasoning more precisely (see chap. III, p. 71 onwards). Evidence for the application of a Bayesian algorithm requires the demonstration that people will adequately evaluate the proportion of elements in the set $D \cap H$ relative to the reference set $D$ when explicitly required to do so (with, e.g., a two-step question). By contrast, it was proposed that evidence for Bayesian reasoning requires the 
demonstration that people can themselves infer that such a proportion is needed in order to evaluate a single-event posterior probability $p(H \mid D)$.

One experiment was designed to investigate this issue. Its purpose was to test the hypothesis that participants would recognise that, in order to evaluate the posterior probability $p(H \mid D)$, they needed to evaluate the proportion of elements in the set $D \cap$ $H$ out of the number of elements in reference set $D$, either before or after having performed a Set-based task framed in either a natural frequency or a partitioned chance format. Additionally, participants' interpretations of a single-event probability statement were recorded to investigate whether it was possible to predict whether they would choose a Bayesian method for estimating $p(H \mid D)$ as a function of the type (frequency vs. single-event) and the format (absolute vs. relative) of the interpretation they provided.

\section{EXPERIMENT 5}

This experiment examined whether participants who provided Bayesian outputs would also demonstrate Bayesian reasoning. Bayesian outputs were elicited using a Setbased posterior probability judgement task either presented in a natural frequency format (Gigerenzer \& Hoffrage, 1995) or in a partitioned chance format (Girotto \& Gonzalez, 2001). Evidence of Bayesian reasoning was assessed with a reasoning task where participants were asked to choose between different methods for evaluating the posterior probability $p(H \mid D)$. Finally, to further investigate people's understanding of single-event probabilities, all participants were also asked to define the meaning of a single-event probability statement.

Consistent with previous research, information format (natural frequencies vs. partitioned chances) was not expected to affect the proportion of judgement outputs coinciding with the Bayesian criterion (Girotto \& Gonzalez, 2001). However, the modal judgement output observed was expected to coincide with the Bayesian criterion, (e.g., Gigerenzer \& Hoffrage, 1995; Girotto \& Gonzalez, 2001). The main objective of this experiment was to test whether Bayesian judgement outputs could be taken as evidence for Bayesian reasoning. If this were true, a positive association could be expected between the judgement output provided in the judgement task and the choice of the method for evaluating a posterior probability in the reasoning task. Finally, Bayesian reasoning was defined as the 'Popperian intuition' that a single-event probability $p(H \mid D)$ can be evaluated on the basis of the proportion of elements in the set $D \cap H$ out of the 
number of elements in the reduced reference set $D$. This intuition was characterised as 'Popperian' in reference to Popper's Propensity theory (see chap. II, p. 25), according to which the probability of a single-event outcome is equal to the relative frequency of occurrence of similar outcomes. This also corresponds to what Teigen (1994) called the "relative frequency rule" (see chap. II, p. 30). This intuition, however, may be mediated by the type (single-event vs. frequency) and the format (absolute vs. relative) of the interpretation given to single-event probabilities. It was therefore hypothesized that the type of reasoning choices (Bayesian vs. non-Bayesian) could be predicted from the type and format of the interpretation of the probabilistic statement.

\section{Method}

\section{Participants}

Participants were recruited using electronic mailing lists addressed to social sciences postgraduate students (e.g., psych-postgrads@jiscmail.ac.uk). A total of eighty Social Sciences students (39 women and 41 men, mean age $=29.3$ years, age range $=19-53$ ) agreed to participate in this experiment. A total of twenty participants were assigned to each of the four experimental conditions.

\section{Design}

A 2 (format: natural frequency vs. partitioned chance) $\times 2$ (order: judgement first vs. reasoning first) between-subjects design was used. There were three dependent measures: the probability judgement, the reasoning method choice and the probability statement interpretation. The order of presentations of the different methods within the reasoning task was fully randomised.

\section{Materials and procedure}

All participants were provided with a single-screen online questionnaire accessible from a computer connected to the World Wide Web. Students were first contacted by email and invited to participate in what was described as a doctoral study. Those who agreed to participate were asked to click on a link provided in the email, which directed them to the questionnaire site. A first general instruction web page indicated that in order to participate in the study, whose duration was estimated at 10 minutes, participants were required to be over 18 and to have not previously participated in the

study. Participants thereafter initiated the experiment by clicking on a button, which 
resulted in the random loading of one of the four versions of the questionnaire so as to ensure that participants were randomly assigned to one of four experimental conditions.

All versions of the questionnaire consisted of three different tasks. In a judgement task, participants were asked to estimate the posterior probability that applicants would obtain the position they applied for, given that they passed a psychometric test, on the basis of base-rate and likelihood information. In a reasoning task, participants were asked to choose between five methods to evaluate the posterior probability that an applicant would fail to get a particular position, given that they failed the psychometric tests. Each method was based on the description of a ratio of cases, defining $p(H), p(D \cap H), p(D)$, $p(D \mid H)$, and the posterior probability $p(H \mid D)$. The reasoning task was framed in terms of failure whereas the judgement task was framed in terms of success, so as to minimise verbal matching. In a final, probability interpretation task, participants were asked to state how they would define the meaning of the conditional probability statement: 'There is a $30 \%$ chance that an applicant will get a position given that he failed the psychometric test'.

For half the participants, the information in the judgement and in the reasoning task was presented in a natural frequency format. For the other half, this information was presented in a partitioned chance format. Within each of these two groups, the order of presentation of the judgement and the reasoning tasks was counterbalanced. The probability interpretation task was the same for all participants and was always given last in the series of three tasks. Figure VI-1 presents the exact content of the three tasks presented in the questionnaire, for each of the two formats used (with the exception of the text presented in brackets, which did not appear in the original text). In the original online version, those three tasks were spread on the screen so that participants could only view and respond to one task at a time. Participants were instructed not to scroll down to view subsequent tasks unless all previous tasks had been completed. 


\section{Frequency format}

I. The company Axellibus requires applicants for a new position to submit to psychometric tests alongside the usual interviews.

In response to the human resources manager's concerns about the reasons why applicants failed to get a position, a report on the relationships between failure of psychometric tests and failure to get a work position was produced. The conclusions of this report are summarised below:

30 out of every 100 individuals who apply for a position will get it.

24 out of the 30 applicants who obtained the position they applied for, had also passed the psychometric tests.

However, 35 out of the remaining 70 applicants who failed to get a position also had passed the psychometric tests.

Consider the case of individuals who apply now. Among those who will pass the psychometric tests, how many will actually obtain the position they applied for? applicants out of applicants.

2. Imagine you want to calculate the chances that individuals will fail to get a particular position, given that they failed the psychometric tests.

You have at your disposal information concerning

- whether applicants failed the psychometric tests

- whether applicants passed the psychometric tests

- whether applicants failed to get a position

- whether applicants got a position

How would you calculate the chances that applicants will fail to get a position, given that they failed the psychometric tests?

$\square$ I would calculate the number of applicants who both failed to get a position and failed the psychometric tests out of the total number of applicants. $[p(D \cap H)]$

$\square$ I would calculate the number of applicants who failed the psychometric tests out of the total number of applicants. $[p(D)]$

$\square$ I would calculate the number of applicants who failed to get a position out of the total number of applicants. $[p(H)]$

$\square$ I would calculate the number of applicants who failed to get a position out of the number of applicants who failed the psychometric tests. $[p(H)$ D)]

I would calculate the number of applicants who failed the psychometric tests out of the number of applicants who failed to get a position. $[p(D \mid H)]$

3. Please, state how you would define the meaning of the following statement:

"There is a $30 \%$ chance that an applicant will get a position given that he failed the psychometric tests."

\section{Chance format}

I. The company Axellibus requires applicants for a new position to submit to psychometric tests alongside the usual interviews.

In response to the human resources manager's concerns about the reasons why applicants failed to get a position, a report on the relationships between failure of psychometric tests and failure to get a work position was produced. The conclusions of this report are summarised below:

Individuals who apply for a position have 30 chances out of 100 of getting it.

24 of the 30 chances of getting the position are associated with a pass in the psychometric tests.

However, 35 out of the remaining 70 chances of failing to get a position are also associated with a pass in the psychometric tests.

Consider the case of an individual who applies now. Among his or her chances of passing the psychometric tests, how many chances will be associated with a successful appointment? _chances out of chances.

2. Imagine you want to calculate the chances that individuals will fail to get a particular position, given that they failed the psychometric tests.

You have at your disposal information concerning:

- their chances of failing the psychometric tests

- their chances of passing the psychometric tests

- their chances of failing to get a position

- their chances of getting a position

How would you calculate the chances that applicants will fail to get a position, given that they failed the psychometric tests?

$\square$ I would calculate the number of chances associated with both failing to get a position and failing the psychometric tests out of the total number of chances.

$\square$ I would calculate the number of chances associated with failing the psychometric tests out of the total number of chances.

$\square$ I would calculate the number of chances associated with failing to get a position out of the total number of chances.

$\square$ I would calculate the number of chances associated with failing to get a position out of the number chances of failing the psychometric tests.

$\square$ I would calculate the number of chances associated with failing the psychometric tests out of the number of chances of failing to get a position.

3. Please, state how you would define the meaning of the following statement:

"There is a 30\% chance that an applicant will get a position given that he failed the psychometric tests."

Figure VI-1 - Questionnaire layout used in Experiment 5 (first order of task presentation) 


\section{Results}

\section{Judgement task}

The judgement output of each participant was classified as either 'Bayesian', 'inverse', 'joint occurrence', 'base rate' or 'other' as a function of the numerical values given by participants in the numerator and the denominator of the ratio provided. Table VI-1 shows the classification grid used.

Table VI-1

Classification grid used to classify outputs.

\begin{tabular}{cccc}
\hline & & \multicolumn{2}{c}{ Expected ratio } \\
\cline { 3 - 4 } Algorithm & Formal equivalent & Numerator & Denominator \\
\hline Bayesian & $p(H \mid D)$ & 24 & 59 \\
Inverse & $p(D \mid H)$ & 24 & 30 \\
Joint occurrence & $p(D \cap H)$ & 24 & 100 \\
Base rate & $p(H)$ & 30 & 100 \\
\hline
\end{tabular}

Participants' judgements were classified accordingly. Judgement outputs that did not match any of the categories in Table VI-1 were classified as 'Others.' Neither the order of task presentation (judgement first vs. reasoning first) nor the format of the information presented (natural frequencies vs. partitioned chances) affected the proportion of Bayesian outputs observed $\left[\chi^{2}(3, N=80)=0.42, p=.94\right]$, therefore the data were collapsed across both order and format of presentation. This suggests that the format of presentation did not affect the proportion of Bayesian judgements elicited, thereby replicating results observed by Girotto \& Gonzalez (2001).

Table VI-2 shows the frequencies of each type of judgement outputs observed.

Table VI-2

Judgement outputs observed.

\begin{tabular}{lccc}
\hline Algorithm & Formal equivalent & Frequency & \% of total \\
\hline Bayesian & $p(H \mid D)$ & 32 & 40 \\
Inverse & $p(D \mid H)$ & 7 & 9 \\
Joint occurrence & $p(D \cap H)$ & 11 & 14 \\
Base rate & $p(H)$ & 13 & 16 \\
Data rate & $p(D)$ & 3 & 4 \\
Others & - & 14 & 18 \\
\hline
\end{tabular}

As expected, the modal judgement output observed coincided with the Bayesian criterion. In fact, the overall percentage of Bayesian outputs observed (40\%) 
corresponded to what is generally observed in Set-based tasks with a partitioned information structure (e.g., Gigerenzer \& Hoffrage, 1995; Girotto \& Gonzalez, 2001; Macchi, 2000).

\section{Reasoning task}

As for the judgement task, the proportions of Bayesian and non-Bayesian reasoning choices were equally likely to occur under each combination of task orders (judgement first vs. reasoning first) and information format (natural frequency vs. partitioned chance), $\left[\chi^{2}(3, N=80)=6.80, p=.08\right]$. Although the number of Bayesian choices observed was slightly higher when the information was presented in a frequency format (18 / 40) than when it was presented in a partitioned chance format $(12 / 40)$, this difference was not significant at the $0.05 \alpha$-level $\left[\chi^{2}(1, N=80)=1.92, p=.17\right]$. The data were therefore collapsed across both task orders and information format. Table VI3 shows the frequencies of each type of reasoning choice observed collapsed across presentation formats and task orders.

Table VI-3

Reasoning method choices observed.

\begin{tabular}{cccc}
\hline Algorithm & Formal equivalent & Frequency & $\%$ of total \\
\hline Bayesian & $p(H \mid D)$ & 30 & 38 \\
Inverse & $p(D \mid H)$ & 13 & 16 \\
Joint occurrence & $p(D \cap H)$ & 25 & 31 \\
Base rate & $p(H)$ & 6 & 8 \\
Data rate & $p(D)$ & 6 & 8 \\
\hline
\end{tabular}

As with judgement outputs, the modal answer was Bayesian as 38\% of participants chose to evaluate the probability $p(H \mid D)$ with the ratio of the proportion of elements in the set $D \cap H$, out of the number of elements in the reduced reference set $D$. Bayesian reasoning was previously defined as one's 'insight' that one needs to base the evaluation of the single-event probability $p(H \mid D)$ on the appropriate ratio of the number of elements in set $D \cap H$ relative to the number of elements in set $D$. Accordingly, if the participants who computed a Bayesian output in the judgement task were Bayesian reasoners, then one would expect that they would also choose the Bayesian reasoning method in the reasoning task. Table VI-4 presents the distribution of participants as a function of their output (Bayesian vs. other) and their reasoning choice (Bayesian vs. other). 
Table VI-4

Cross-tabulation of judgement output and reasoning choice

\begin{tabular}{lccc} 
& \multicolumn{2}{c}{ Judgement output } & \\
\cline { 2 - 3 } Reasoning choice & Bayesian & Other & Total \\
\hline Bayesian & 18 & 12 & 30 \\
Other & 14 & 36 & 50 \\
Total & 32 & 48 & 80 \\
\hline
\end{tabular}

In fact, only 18 out of 80 (22.5\%) participants both provided a Bayesian output and chose the Bayesian reasoning method for computing $p(H \mid D)$ whereas 26 out of 80 (32.5\%) participants were inconsistent; that is, either provided the Bayesian output but did not choose the Bayesian method or chose the Bayesian method but did not provide a Bayesian output. There was no association between inconsistency and the type of Bayesian outcome (judgement or reasoning), [McNemar $\left.\chi^{2}=.04, p=.85\right]$. In other words, the proportion of inconsistency observed when participants provided a Bayesian output did not differ from the proportion of inconsistency observed when participants chose the Bayesian reasoning method.

A significant association between providing a Bayesian judgement output and choosing the Bayesian reasoning choice was observed [Likelihood $\chi^{2}(1, N=80)=$ 8.01, $p=.005$ ]. A measure of agreement, however, showed that the degree of consistency observed between judgement and reasoning outcomes was far from being perfect, with a Cohen's $\kappa$ value of .31 . This was also true for the full matrix of judgement outputs and reasoning choices [omitting judgements that had no corresponding reasoning choice option, see Table VI-5]: although the consistency observed was better than what would be expected by chance, at the $.05 \alpha$-level, the Cohen's $\kappa$ value of .20 revealed that the consistency between the type of judgement output and the reasoning method chosen was rather low. 
Table VI-5

Matrix for evaluating the consistency of judgement outputs and reasoning choices.

\begin{tabular}{lrrrrrr}
\hline & \multicolumn{5}{c}{ Judgement } & \\
\cline { 2 - 5 } Reasoning & $p(H)$ & $p(D \mid H)$ & $p(D \cap H)$ & $p(D)$ & $p(H \mid D)$ & Total \\
\hline$p(H)$ & 2 & 3 & & & 1 & 6 \\
$p(D \mid H)$ & 1 & 1 & 1 & 1 & 5 & 9 \\
$p(D \cap H)$ & 4 & 2 & 7 & 1 & 7 & 21 \\
$p(D)$ & 3 & & & & 1 & 4 \\
$p(H \mid D)$ & 3 & 1 & 3 & 1 & 18 & 26 \\
Total & 13 & 7 & 11 & 3 & 32 & 66 \\
\hline
\end{tabular}

Note. $N=66$ because the judgements that did not correspond to any of the reasoning methods proposed were not included in this analysis.

\section{Probability interpretation task}

In this last task, participants were asked to state how they would define the meaning of the statement "There is a 30\% chance that an applicant will get a position, given that he failed the psychometric tests". Answers were classified by two independent coders according to two different criteria; namely, the type of probability (single-event, frequency, ambiguous/other), and the information format (absolute, relative, other/ambiguous). Since the second independent coder was not familiar with these concepts, the coding scheme for the probability type proposed to distinguish between interpretations referring to 'a number of individuals' (frequency), 'a number of chances' (single-event) and those with another/ambiguous referent. Similarly, the coding scheme for the information format asked to distinguish between interpretations based on 'a percentage, e.g., 10\%' (relative), 'a number of instances, e.g., 10 out of 100' (absolute) and those based on another/ambiguous numerical format.

Agreement for both criteria was significantly better than chance and showed rather high inter-rater reliability (Cohen's $\kappa=0.79$ for the probability type criterion, and Cohen's $\kappa=0.90$ for the information format criterion). Remaining cases of disagreement were resolved through discussion. All participants' statements are presented in Appendix B (p. 278). Table VI-6 presents the frequencies of observations for each type of probability (frequency, single-event, ambiguous/other) as a function of information format (absolute, relative, ambiguous/other). 
Table VI-6

Frequencies of observation of each combination of probability type and information format.

\begin{tabular}{ccccc}
\hline & \multicolumn{3}{c}{ Probability type } & \\
\cline { 2 - 4 } Information Format & Frequency & Single-event & Ambiguous & Total \\
\hline Absolute & 23 & 13 & 3 & 39 \\
Relative & 11 & 15 & 1 & 27 \\
Ambiguous & 1 & 4 & 9 & 14 \\
\hline Total & 35 & 32 & 13 & 80 \\
\hline
\end{tabular}

Out of the 80 participants' statements collected, a total of 35 (44\%) were expressed using a frequency probability type, 32 (40\%) were expressed using a single-event probability type, whereas the rest were ambiguous. The proportions of frequency and single-event types were not significantly different $\left[\chi^{2}(1, N=67)=.13, p=.71\right]$. Interpretations based on a frequency probability type in an absolute information format were the most commonly observed. Since the probability statement used was a base-rate statement, absolute frequency and natural frequency formats were confounded. This result can thus be taken as being consistent with the argument that natural frequency formats are the most natural representation of probability (Gigerenzer \& Hoffrage, 1995; Hoffrage et al., 2002). Yet, this result was not statistically significant. Participants were indeed as likely to have provided an interpretation based on this combination of probability type and information format as any other (i.e., absolute single-event, relative frequency, or relative chance) $\left[\chi^{2}(3, N=62)=5.35, p=.15\right]$.

The last hypothesis tested in this experiment was that participants' interpretation of the single-event probability statement presented in the final task would predict whether they would demonstrate Bayesian reasoning in the reasoning task. This prediction was based on the conjecture that Bayesian reasoning was underpinned by the Popperian intuition that a single-event probability can be evaluated on the basis of a relative frequency. To control for ambiguity, the 18 ambiguous interpretations (see Table VI-6) were discarded from the analysis, and the remaining interpretations $(N=62)$ were classified as absolute frequency, absolute single-event, relative frequency or relative single-event. Table VI-7 presents the distribution of participants who chose a Bayesian reasoning method as a function of the type and format of their interpretation of the single-event probability statement. Contrary to what was hypothesised, there was no association between the proportion of Bayesian reasoning choices observed and the type 
and format of the interpretations given for the single-event probability statement. A logistic regression analysis with the reasoning choice as dependent variable (Bayesian, other) and the type (frequency, single-event) and format (absolute, relative) of the interpretation of the single-event probability statement as predictor variables was applied to the data. The test of the full model (including all interactions) versus a model with intercept only was not statistically significant $\left[\chi^{2}(2, N=62)=0.33, p=.85\right]$ showing that, contrary to what was hypothesized; Bayesian choices were not related to the type or format of the interpretation of the single-event probability statement.

Table VI-7

Frequencies of Bayesian reasoning choices observed as a function of the type and format of the single-event probability statement interpretation.

\begin{tabular}{lccccc}
\hline & \multicolumn{4}{c}{ Type } \\
\cline { 2 - 5 } & \multicolumn{2}{c}{ Frequency } & \multicolumn{2}{c}{ Single-event } & \\
\cline { 2 - 5 } Reasoning choice & 9 & 3 & 3 & 5 & 20 \\
\cline { 2 - 5 } & Absolute & Relative & Absolute & Relative & Total \\
\hline Bayesian & 14 & 8 & 10 & 10 & 42 \\
Non-Bayesian & 23 & 11 & 13 & 15 & 62 \\
Total & & &
\end{tabular}

\section{Discussion of Experiment 5}

The purpose of this experiment was to investigate whether judgement outputs that coincide with the Bayesian criterion in partitioned Set-based tasks provide evidence for Bayesian reasoning. Bayesian reasoning was defined as the inference that what is needed in order to evaluate the posterior probability $p(H \mid D)$ is the ratio of the number of elements in the set $D \cap H$ to the number of elements in the reduced reference set $D$ (whether such elements are concrete instances or abstract chances). Evidence for Bayesian reasoning was assessed by asking participants to choose between different reasoning methods (describing different types of ratios, including the Bayesian ratio defined above) to compute the posterior probability $p(H \mid D)$. Bayesian judgement outputs were elicited by using partitioned Set-based tasks presenting the information in either a natural frequency format or a partitioned chance format.

Results showed that the proportion of judgement outputs which coincided with the Bayesian criterion was not affected by the type of information (natural frequency vs. partitioned chance), thereby replicating findings observed by Girotto and Gonzalez (2001) who demonstrated that natural frequency formats were not necessary to elicit 
Bayesian judgements. In support of the second hypothesis tested, the modal output elicited with the partitioned tasks coincided with the Bayesian criterion. The overall proportion of Bayesian judgements observed was $40 \%$, which is consistent with the performance rates observed in previous studies (Gigerenzer \& Hoffrage, 1995; Girotto \& Gonzalez, 2001; Macchi, 2000). The modal choice in the reasoning task was also the Bayesian method (38\%).

Although there was a significant association between Bayesian judgement output and Bayesian reasoning choices, the size of this association was rather low (Cohen's $\kappa=$ .31). This result suggests that the assumption that the observation of Bayesian judgement provides evidence for Bayesian reasoning is not warranted: only half the participants whose judgement output coincided with the Bayesian criterion and half of those whose reasoning choice coincided with the Bayesian method (i.e., 23\% of the total sample of participants) were consistent; that is, provided the Bayesian answer, in both the judgement task and in the reasoning task.

As previously mentioned (see chap. III, p. 73), one of the assumptions made by the researchers who first developed the Set-based judgement paradigm for the study of posterior probability judgements, is that the natural frequency format leads to high rates of Bayesian performance because humans have evolved cognitive systems that are tuned to natural frequencies acquired by natural sampling rather than to probabilities or relative frequency (Gigerenzer \& Hoffrage, 1995). The interpretations of the singleevent statement, "There is a 30\% chance that an applicant will get a position, given that he failed the psychometric tests," observed did not support the hypothesis that natural frequencies were a more natural format of interpretation. In fact, the proportions of different interpretations observed were equally distributed among four target categories, namely "absolute frequency", "absolute single-event", "relative frequency", and "relative single-event." The last hypothesis tested whether there was an association between the type and format of the interpretation provided by participants and the choice of reasoning method observed (Bayesian vs. non-Bayesian). This hypothesis was not supported by the data. This result shows that participants' choice of the Bayesian reasoning method in the reasoning task was independent of their interpretation of single-event probability statements. The type and format of the interpretations elicited, however, may have been related to people's background knowledge rather than their ability to relate single-event probabilities to ratios of frequencies. 
Harries and Harvey (2000) indeed suggested that people store different types of likelihood information about events, depending on their personal experience of those events. Accordingly, likelihood information about events such as causes of deaths would be stored in terms of relative frequencies because this information is obtained from public sources rather than derived from personal experience. In contrast, personally experienced events, such as students missing a lecture would be stored in terms of natural frequencies or event counts. In a similar vein, one could argue that individuals will make different interpretations of likelihood information depending on their experience of these events. Thus, the different types of interpretations observed in the last task could be attributed to individuals' past experiences whereby individuals who had experienced the event described, would be more likely to interpret likelihood information in a natural frequency format than those who had had little personal experience of this event.

Altogether, these results suggest that one cannot assume that Bayesian outputs observed in Set-based tasks are underlain by Bayesian reasoning, although further research is still needed to substantiate the results observed. The reasoning task used to provide evidence for Bayesian reasoning evaluated whether participants would identify the appropriate ratio for the evaluation of the posterior probability $p(H \mid D)$, when they are explicitly prompted to rely on frequencies of observations (when the information was presented in a natural frequency format) or discrete sets of chances (when the information was presented in a partitioned chance format) and when they are presented with an array of reasoning methods. A related issue in identifying whether participants can demonstrate Bayesian reasoning would be to demonstrate whether participants can infer that they need to evaluate such a ratio, when they are not prompted to use frequencies or chances of observations, and in the absence of reasoning method exemplars. This would amount to demonstrating that participants would 'naturally' rely on natural frequencies or partitioned chances to estimate a single-event probability, even when they are not prompted to do so.

Another issue concerns the extent to which participants are capable of overt Bayesian reasoning. The reasoning task used required the conscious inference that $p(H \mid D)$ needed to be estimated by the ratio $n(D \cap H) / n(D)$. Someone who does not demonstrate Bayesian reasoning in such a task may well be able to produce an accurate estimate for $p(H \mid D)$ when sampling cases from samples defined by $D \cap H$ and $D \cap \sim H$, 
respectively (e.g., see Fiedler, 2000). Yet, whether such accurate sampling would demonstrate Bayesian reasoning is arguable. If one defines reasoning as the forming of judgements using careful arguments (see chap. III, p. 67), sensu stricto, a process that is not overt cannot be qualified as reasoning. Moreover, the ability to form accurate judgements from direct sampling does not take away the importance of evaluating whether and when individuals are capable of demonstrating Bayesian reasoning in situations that do not involve direct sampling but instead present the information as a summary of natural frequencies (or partitioned chances) of occurrences resulting from the sampling cases from samples defined by $D \cap H$ and $D \cap \sim H$, respectively, as it is the case in Set-based tasks. Not all $p(H \mid D)$ judgements can be based on actual sampling and a correct answer based on summary information does not guarantee Bayesian reasoning. For example, when jurors are asked to evaluate the number of individuals who were found guilty among those who presented a DNA match, they may provide the 'correct' frequency estimate. However, unless they understand and recognise that their frequency estimate corresponds to the probability of the particular defendant they are judging being guilty given that his DNA matched the sample found at the crime scene, their accurate frequency judgement is of little pragmatic value (cf. Koehler, 2001).

\section{CONCLUSIONS}

This chapter covered the last of the three experimental paradigms within which posterior probability judgements are currently investigated. As for the two preceding experimental chapters of this thesis, it was the undertaking of a process-focused perspective that led to the identification of the research issues thereafter investigated. The process-focused approach calls for the development of a systematic description of the judgement methods underlying observed outputs, to form the basis of a theoretical account of the cognitive processes that underpin the application of such methods.

The results observed within the Set-based judgement paradigm undeniably demonstrate that the tasks used in this paradigm can lead people to provide Bayesian outcomes. The subsequent conclusion that such observations provide evidence for Bayesian reasoning was, however, questioned. The results obtained in the present study show that this conclusion is generally not warranted when evidence for Bayesian reasoning is taken to be the consistency of Bayesian answers in both a Set-based 
judgement task and in a Bayesian reasoning task [where participants were asked to identify the appropriate method for evaluating the single-event probability $p(H \mid D)]$.

But, even if the results observed in Set-based partitioned judgement tasks do not necessarily provide evidence for Bayesian reasoning, these tasks do generally encourage the application of a Bayesian algorithm, i.e., lead individuals to evaluate the ratio of the proportion of elements in the set $D \cap H$, out of the number of elements in the reduced reference set $D$. From a purely cognitive perspective, this raises the question of the cognitive processes that may have entailed the application of such an algorithm. The review of existing algorithmic accounts of posterior probability judgements observed in Set-based tasks revealed that all but one account only provided a superficial explanation of the cognitive processes that may be at work in these tasks. The only exception was the Mental Model Theory of naïve probabilistic reasoning, which provided the most comprehensive algorithmic account of probability judgements to date. Nevertheless, it was argued that MMT still suffers from some important shortcomings with regards to the account of posterior probability judgements. In the following chapter, these shortcomings are addressed by a new algorithmic account, which addresses the theoretical issues identified in both the Textbook-problem paradigm and the Set-based judgement paradigm.

\section{sk}


Une représentation met en jeu une relation entre trois termes: quelque chose qui représente, quelque chose qui est représenté, et un dispositif de traitement de l'information qui utilise la représentation.

(A representation entails a relationship between three terms: something that represents, something that is represented, and an information processing device, which uses the representation)

(Sperber, 1996, p. 85)

The ultimate objective of the process-focused approach favoured in this thesis is to develop an algorithmic account of posterior probability judgements.

Such an account should explain how the mental representation of the task and its processing entail the application of the computational judgement methods previously identified. The review of the literature led to the identification of several such accounts purporting to explain the origin of the application of judgement methods such as the representativeness heuristic or the inverse fallacy. The empirical work carried out within the Social-judgement paradigm did not permit the unambiguous identification of the method or methods underlying judgement outputs observed with Social-judgement tasks. This, in turn, impinges on the development of an algorithmic account of judgements within this paradigm (see chap. IV, p. 132). By contrast, reliable computational accounts were identified within both the Textbook-problem paradigm and the Set-based paradigm. In the Textbook problems, the inverse fallacy was identified as the most common method being applied (see chap. V). In Set-based tasks, it was confirmed that partitioned Set-based tasks elicit the application of a Bayesian algorithm (see chap. VI). The review of corresponding algorithmic accounts in these two paradigms, however, revealed that these accounts were subject to several shortcomings (see chap. III).

Within the literature on the Textbook-problem paradigm, three main accounts were identified. Namely, the causality account (Tversky \& Kahneman, 1980), the relevance account (Bar-Hillel, 1980), and the partitive account (Macchi, 1995). None of these accounts, however, comprehensively explains how the processing of the task mediated the different judgements observed. Within the Set-based paradigm, two types of explanatory accounts were identified. The original account was based on the evolutionary assumption that organisms have evolved mechanisms that are tailored to the structure of their external environment (Gigerenzer \& Hoffrage, 1995; see also Cosmides \& Tooby, 1996). This account focused on the role of the external representation of the information. But, it does not explain how the internal 
representation of Set-based tasks mediates the judgements observed. In other words, those mechanisms shaped by evolution still remain to be specified. Alternative explanations generally relied on the assumption that the elicitation of Bayesian answers was mediated by the task structure (Macchi, 2000), itself mediating the mental representation of the task (e.g., Evans et al., 2000; Girotto \& Gonzalez, 2001; Mellers \& McGraw, 1999; Sloman et al., in press). However, most of these accounts never specified how the external structure of the task was mentally represented, or how and why such representation may or may not elicit a Bayesian judgement output.

The only exception is provided by the Mental Model Theory (MMT) of probabilistic reasoning (Johnson-Laird et al., 1999; Girotto \& Gonzalez, 2001). This theoretical account was aimed at formalising the relationship between the mental representations elicited from the task and the judgement observed. It made convincing predictions for extensional judgements of probabilities involving assessments of discrete possibilities or chances. Yet, it was contended that the MMT's account of posterior probability judgements, invoking the application of the subset principle, did not consistently explain the judgements observed in the tasks that are the focus of the present thesis (see chap. III, p. 74 onwards).

The purpose of the present chapter is to introduce a new theory, called Sample Space Representation (SSR) theory. SSR theory differs from MMT on two main grounds: firstly, whereas MMT suggests that any probability statement will be represented with models of individual elements, SSR theory proposes that the distinction between such elementary representations and sample representations is of key importance. Secondly, whereas MMT only opposes extensional and nonextensional probability judgements (the latter referring to judgements based on subjective beliefs), SSR theory distinguishes extensional and intensional properties of the representation of any probability statement and shows how both can determine the final judgement outcome.

Following a general overview of the theory, its specific propositions are presented. These propositions unequivocally define the exact processes by which different sample space representations are elicited by different tasks as well as the processes by which judgements are elicited. In the subsequent section, it is shown that, on the basis of the specific propositions of the SSR theory, one can explain how different tasks used both within the Textbook-problem and the Set-based paradigm (including the tasks used in this thesis) lead to the different judgements observed; thereby positing the SSR theory as 
a general algorithmic account of posterior probability judgements. The chapter concludes with a discussion of both theoretical and empirical implications of SSR theory for the study of posterior probability judgement within each of these two paradigms.

\section{THEORY}

\section{General presentation}

The Sample Space Representation theory, akin to the Problem Solving theory (Newell \& Simon, 1972) and the Mental Models theory (Johnson-Laird, 1983), for instance, is a representational theory. Specifically, it is based on the assumption that individuals' probability judgements are guided by their mental representation of the statements in probability judgement tasks, called the sample space representation. Such a representation is assumed to be initiated by the processing of the first statement of the task. The initial representation is assumed to be dynamic and updated as more information becomes available for processing. The final sample space representation, upon which the judgement is based, is assumed to be determined by the task environment. The concept of task environment was originally introduced by Newell and Simon (1972) as

....an environment coupled with a goal, problem, or task-the one for which the motivation of the subject is assumed. It is the task that defines a point of view about an environment, and that, in fact, allows an environment to be delimited. (p. 55)

The task's goal or goals are assumed to elicit the application of a judgement method to the existing sample space representation. The judgement output can thus be conceptualised as the result of the application of the judgement method to the representation of the task. The term 'judgement method' shall be used instead of 'judgement heuristic', to refer to a more specific class of judgmental processes, namely, processes involving the search of the available representation for a model, sample, or samples whose specific descriptive units (later defined as 'intensions') match those of the task goal statement. The top panel of Figure VII-1 briefly outlines these three main processing stages (i.e., initial sample space representation, sample space updating, and judgement), using flow diagrams. The cognitive processes involved in each of these stages will be reviewed in greater detail in the next sections of this chapter. 
Probability judgement

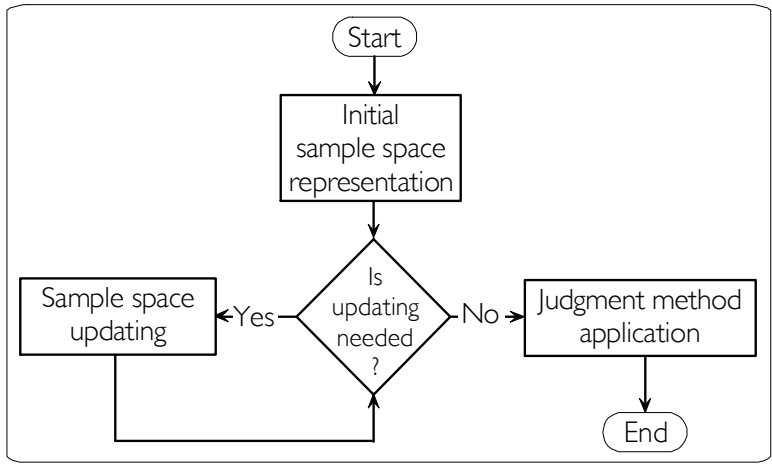

Problem solving

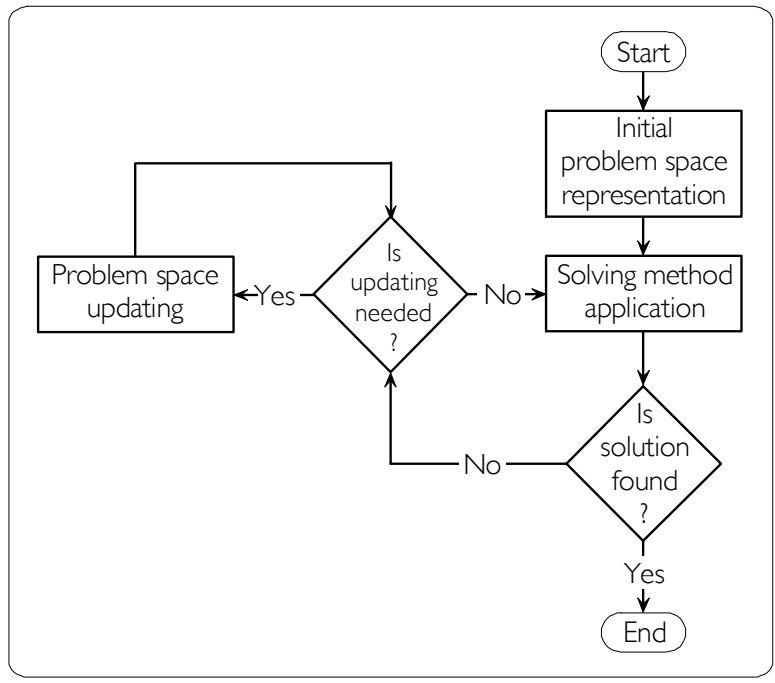

Figure VII-1 - Flow diagrams comparing the processes involved in probability judgement and problem solving.

Note. The arrows indicate the sequence in which processes are executed.

Although the processes assumed to be at work in probability judgement tasks are similar to those posited by Problem Solving theory (Newell \& Simon, 1972), there are some important features that distinguish the two accounts. Within a problem-solving context, the processes involved consist in the representation of the problem space, followed by the application of a sequence of operations to the initial situation to transform it into the goal situation. Newell and Simon (1972) defined individuals' problem space as including (i) states of knowledge about the task, (ii) operators or rules that produce new states of knowledge from existing ones, (iii) a problem including a set of final states of knowledge (goal) to be reached by applying the operators, and (iv) the total knowledge available in a given knowledge state, including temporary dynamic information, the knowledge state itself, etc. (see Newell \& Simon, 1972, pp. 810-811). For instance, in a chess game, the states of knowledge are the current positions of the 
chess pieces, the operators are defined by the possible moves of the pieces, and the goal of the problem is related to checkmate.

The term 'sample space representation', as it shall be used herein thus refers to a concept that is somewhat different from Newell and Simon's (1972) concept of task environment representation or problem space. Moreover, in cases where the goal is to produce a probability judgement, SSR theory assumes the elementary processes involved differ slightly. The bottom panel of Figure VII-1 illustrates the elementary processes involved in problem solving tasks. In those tasks, individuals seek to unravel a hidden solution through the representation of the problem space, followed by the recurring application of solving methods constrained by the operators defined in the problem statement (Newell \& Simon, 1972). By contrast, in probability judgement tasks, individuals are required to produce a unique judgement on the basis of the information contained in the task. Whereas problem solvers can reiterate the problem solving procedure until they find a satisfactory answer (i.e., one that matches the goal state), judgement makers have no means to assess the validity of their judgement.

The following section presents the propositions of SSR theory, which will in turn form the grounds for the formalisation of (i) the processes underlying the representation of the judgement task sample space, and (ii) the ways in which judgement methods are applied to this representation.

\section{Initial sample space representation}

\section{Type and intensions of task statements}

According to SSR theory, the processing of the first statement of the task leads to an initial sample space representation. The content of this initial sample space representation is determined by the type of this statement. More specifically, the first proposition of the theory is as follows,

Proposition 1a. The structure and content of the initial sample space representation are determined by the type (elementary vs. set) of a given statement and its intensions (i.e., the specific descriptive units defining its components).

Elementary statements refer to a unique element. For example,

[1] Mary who is happy,

[2] A cat that is playing,

are both elementary statements. In contrast, set statements refer to sets of elements. For instance, statements such as,

[3] The children who are joyful, 
[4] The cats that are sleeping,

refer to sets composed of at least two elements. Intensions or defining properties refer to the specific way in which elements are characterised. For instance, in statement [1], the element "Mary" is characterised by the intension 'happy'. Intensions are not based on surface features. For example, the statements

[5] A woman with breast cancer.

[6] A woman who has breast cancer.

both define two characteristics of an element $x$, namely 'is a woman' and 'has breast cancer'. Intensions should not be confused with meanings: synonyms such as 'happy' and 'joyful' have identical meanings, yet they represent distinct intensions, which may or may not be interpreted as being identical. Formally, the subject of an elementary statement will be denoted by $x$, and its intension, by $i(x)$. For example, let $x_{0}$ denote Mary, and let $i_{1}(x)$ be the intension ' $x$ is happy'; Statement [1] can thus be formally represented by $i_{1}\left(x_{0}\right)$.

Set statements refer to sets of elements also characterised by their intensions. For example, statement [3] refers to the set of elements characterised by $i_{1}(x)$ and $i_{2}(x)$ where $i_{1}(x)$ is the intension ' $x$ is a child' and $i_{2}(x)$ is the intension " $x$ is joyful." Using the set theoretic notation (see chap. II, p. 14), statement [3] can thus be formally represented by $\mathscr{S}=\left\{x \mid i_{1}(x) \wedge i_{2}(x)\right\}$ where the symbol " $\wedge$ " represents the logical connector "and," and the notation reads "The sample space $\mathscr{S}$ defined by the set of elements $x$ such that $x$ is described by intensions $i_{1}$ and $i_{2}$ ”. Intensional definitions of sets contrast with extensional definitions. For example, the intensional description of the set of elements that are dogs would be based on the description of the distinctive features or characteristics of this animal. By contrast, the extensional description of this set would include all breeds to which to word 'dog' is applied (Chalker \& Weiner, 1994).

Set statements can also define a reference set and its subset. For example, consider the following statement,

[7] Only the cats that are black are also sleeping.

This statement defines both a reference set of elements (i.e., the elements characterised as cats) and a subset of elements within this reference set (those characterised as black and sleeping). Statement [7] can thus be formally represented by the reference set $\mathscr{S}=$ $\left\{x \mid i_{1}(x)\right\}$ where $i_{1}(x)$ is the intension " $x$ is a cat," and the subset $S$, included in $\mathscr{S}$, is characterised by $S=\left\{x \mid i_{1}(x) \wedge i_{2}(x) \wedge i_{3}(x)\right\}$ where $i_{2}(x)$ is the intension " $x$ is black" and $i_{3}(x)$ is the intension " $x$ is sleeping." 


\section{Representation of elementary statements}

The ways in which elementary statements and their intensions will mediate the mental representation of the task are summarised in the following proposition,

Proposition $1 \mathrm{~b}$. The element defined by an elementary statement is represented by a single element, characterised by the intension(s) defined in the statement, and (if applicable) the probability associated with those intension(s).

For example, the following statement,

[1] Mary who is happy,

corresponds to the graphical representation (1), characterised below,

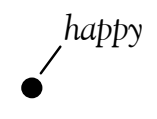

More formally, the unique element 'Mary' can be denoted by $x_{0}$ and its intension 'is happy', by $i_{1}(x)$. Thus, the representation of the statement 'Mary is happy' can be formally represented by (1)'

$$
\exists x_{0}, i_{1}\left(x_{0}\right)
$$

which reads 'there is an element $x_{0}$ such that $x_{0}$ is described by intension $i_{1}$ ' where $i_{1}(x)=$ " $x$ is happy."

For simplicity purposes, representations will be characterised graphically throughout. Both formal and graphical representations, however, should be understood as symbolic mediums for characterising actual representations. The modalities by which such representations are instantiated in individuals' minds are beyond the scope of this thesis; their characterisation belongs to the "implementational level of analysis" (Marr, 1982) whereas SSR theory belongs to an "algorithmic level of analysis" (ibid.).

\section{Representation of set statements}

The following proposition summarises the ways in which set task statements and their intensions will mediate the mental representation of the task. By convention, the expressions 'reference set' and 'subsets' will be used to denote the sets defined in the written task statements. In contrast, the expressions 'sample space' and 'sample' will be used to denote the corresponding mental representation of these reference sets and subsets, respectively. 
Proposition 1c. The reference set defined by a set statement is represented by a sample space of elements. When the set statement also refers to a subset of the reference set, this subset is represented by a sample included in the sample space. Both sample space and sample are characterised by their respective intension(s), as defined in the set statement, and (if applicable), by either the probability of the sample in reference to the sample space or the respective sizes of sample and sample space.

For example, statement [3],

[3] The children who are joyful,

corresponds to the representation (3) characterised below,

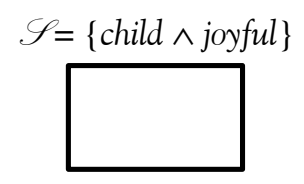

which denotes the reference set of elements that are children and are joyful. More formally, this statement can be represented by,

$$
\mathscr{S}=\left\{x \mid i_{1}(x) \wedge i_{2}(x)\right\}
$$

$i_{1}(x)=$ ' $x$ is a child'

$i_{2}(x)=$ ' $x$ is a joyful'

which defines the reference set of elements $x$ such that $x$ is described by $i_{1}(x)$, defining the intension ' $x$ is a child' and $i_{2}(x)$, defining the intension ' $x$ is joyful'.

Now consider statement [7],

[7] Only the cats that are black are also sleeping.

This statement defines both a reference set ('the cats') and a subset of this reference set ('the cats that are black and are sleeping'). Accordingly, Proposition 1c suggests that this statement will entail the following representation,

$$
S=\{\text { cat } \wedge \text { black } \wedge \text { sleeping }\}
$$

\section{Representation of probability statements}

Proposition $1 \mathrm{~b}$ and $1 \mathrm{c}$ entail that probability statements will lead to different representations, depending on whether they are characterised as elementary or set statements. For example, the following statement,

[8] There is a $10 \%$ probability that a woman has breast cancer, expresses the possibility or the chance that an individual woman has breast cancer. Such a statement is likely to be represented by a unique element described by the intension 'is 
a woman' and with a $10 \%$ probability of being described by intension 'breast_cancer.' Accordingly, the graphical representation of statement [8] will be characterised by,

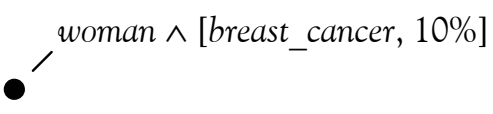

Where the notation '[breast_cancer, 10\%]' is used to denote the fact that there is a $10 \%$ probability that the element is described by intension 'breast_cancer.' This representation is consistent with the Mental Model account of naïve probability judgements (JohnsonLaird et al., 1999), although the notation used here differs slightly from the notation used in MMT. One of the key principles of MMT is that mental models represent only what is true in a statement. SSR theory makes a similar assumption albeit restricted to the initial representation of a statement. Namely, SSR theory assumes that the mental representation of the statement will initially include only what is explicitly stated in the statement (this distinction will ultimately allow SSR theory to account for individual variability in judgement outputs, see p. 206).

But, there is a critical distinction between the MMT account and the SSR account. The MMT account posits that individuals' representations will always be based on individual mental models. Thus, according to MMT, a set statement (i.e., a statement referring to a set of elements), will be represented by a series of models, each model representing an element of the set. By contrast, the distinction between representations based on elementary and set statements is of key importance to SSR theory. For example, consider the following set statement,

[9] $10 \%$ of women have breast cancer,

This statement refers to the subset of elements that are women and have breast cancer within the reference set of elements that are women. According to Proposition 1c, the representation of such a statement would therefore be based on the sample space $\mathscr{I}_{1}$ defined by $\mathscr{S}_{1}=\{$ woman $\}$. Included in $\mathscr{S}_{1}$, will be the sample $S_{1}$, defined by $S_{1}=\{$ woman $\wedge$ breast_cancer $\}$. Finally, the representation will include the probability of $S_{1}$, relative to $\mathscr{S}_{1}$, that will be denoted as $p\left(S_{1} \mid \mathscr{S}_{1}\right)=10 \%$. This notation can be mapped onto the more common notation used to refer to the probability that a hypothesis is true, $p(H)$. If " $x$ has breast cancer" was an hypothesis, $p(H)$ would correspond to the proportion of elements in a given sample space that have the intension " $x$ has breast_cancer," namely, $p(H \mid \mathscr{S})$. The latter notation simply explicitly states the referent of the probability. In short, statement [9] can be formally represented as follows, 


$$
\begin{gathered}
\multicolumn{2}{c}{\mathscr{S}_{1}=\{\text { woman }\}} \\
S_{1}=\{\text { woman } \wedge \text { breast_cancer }\} \\
p\left(S_{1} \mid \mathscr{S}_{1}\right)=10 \%
\end{gathered}
$$

Absolute frequency statements would also entail a sample representation. For example, a statement such as "10 out of 100 women have breast cancer" would elicit a representation similar to representation (9), but would feature $n\left(S_{1}\right)=10$ and $n\left(\mathscr{S}_{1}\right)=$ 100 instead of $p\left(S_{1} \mid \mathscr{S}_{1}\right)$. Similarly, a statement such as "10 out of 100 chances are associated with breast cancer" would elicit a representation identical to the previous statement but with $\mathscr{S}_{1}=\{$ chance $\}$ and $S_{1}=\{$ chance $\wedge$ breast_cancer $\}$.

\section{Overview of initial representation stage}

In sum, SSR theory proposes that the first stage of processing entailed by Textbook and Set-based problems is the construction of an initial representation, which is mediated by the type and format of the first statement that is presented in the task. Figure VII-2 presents a flowchart summarising the processes that may be involved in the elaboration of the initial representation. Once this initial representation is built, SSR theory assumes that it will be updated with the information contained in subsequent task statements. The exact modalities of this second stage are presented in the following section. 
Initial sample space representation

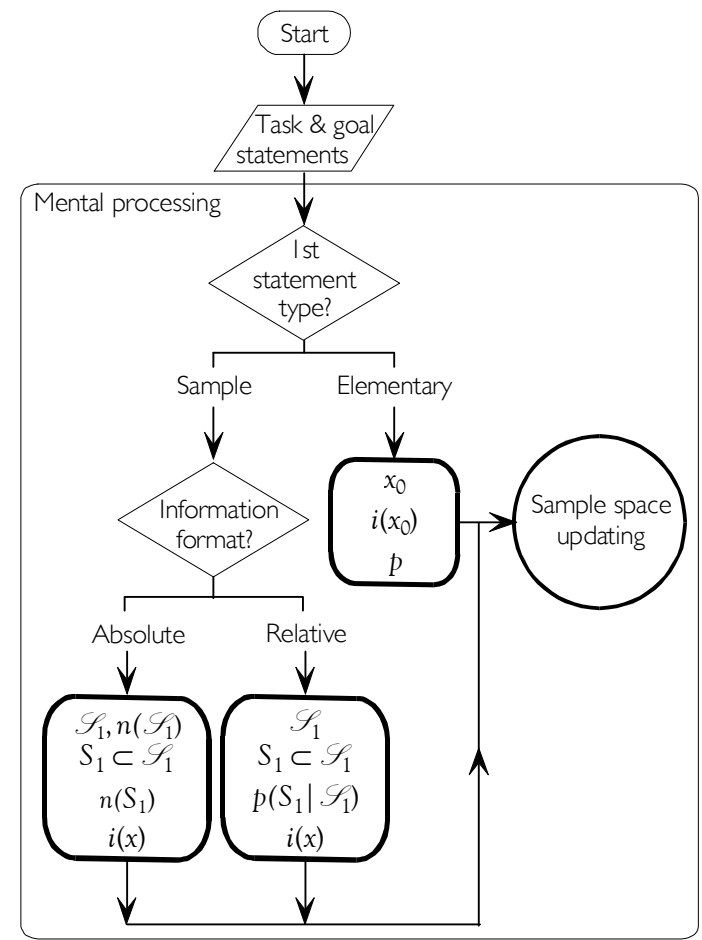

Key

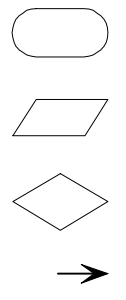
the system.

Input/Output: Represents information entering or leaving the system.

Branching point: indicates conditional alternative paths.

Flow: Indicates the sequence and direction of the flow.

Figure VII-2 - Flow diagram summarising the processes involved in the elaboration of the initial representation of a posterior probability judgement task.

Note. The flowchart represents all the processes that are potentially at work in representing the initial statement of the task. The flow followed by a given individual processing the task is determined by the statement and the probability format of the information in this initial statement.

\section{Sample space updating}

As noted earlier, representations are assumed to be dynamic. This assumption is captured by Proposition 2a of the SSR theory,

Proposition 2a. If the task contains more than one statement, the existing representation will be updated as the judge processes more information.

The ways in which the existing representation are updated depends on the type of the new statement processed (Proposition 1b).

\section{Updating elementary representations}

The representation of elementary statements will either be updated with new intensions or with new elements altogether, 
Proposition $2 \mathrm{~b}$. Provided that an element $x_{i}$ has been defined by a previous elementary statement, its intensions will be updated to include the intensions defined in a new elementary statement if the following conditions are satisfied:

(i) There is an intensional cue: The intensions of the element $x_{i+1}$ match those of element $x_{i}$, thereby indicating that $x_{i+1}$ is an element similar to $x_{i}$.

(ii) There is a determinative cue: A definite determiner is used to indicate that the element $x_{i+1}$ is the same element as the $x_{i}$ element.

We already saw that intensional cues define the distinctive features of an element. Determinative cues are relational cues. They serve to identify the reference of an element. Consider the following statements,

[10a] A cab was involved in an accident.

[10b] A witness identified the cab involved in an accident as a Blue cab.

Statement [10a] defines a singular element $x_{0}$ with intensions " $x$ is a cab" and " $x$ is involved in an accident," and would therefore be represented as follows (Proposition 1b),

$$
\bullet^{\prime c a b \wedge i n \_a c c i d e n t}
$$

Statement [10b] refers to an element $x_{1}$ with intensions " $x$ is cab," " $x$ is involved in an accident," and " $x$ was identified as a Blue cab by the witness." Since both $x_{0}$ and $x_{1}$ are described by intensions " $x$ is a cab" and " $x$ is involved in an accident," condition i of Proposition $2 \mathrm{~b}$ is satisfied. Additionally, the presence of a demonstrative article 'the' in statement [10b] indicates that $x_{1}$ is indeed the same element as $x_{0}$, thereby satisfying condition ii of Proposition $2 b$. It follows that the representation (10a) will be updated as follows,

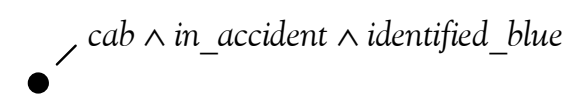

Thus, the presence of both an intensional and a determinative cue will guarantee the updating of the intensions of an existing element, as opposed to the representation of two distinct elements. The presence of a determinative cue would be necessary to unambiguously convey that the intensions of an existing element should be updated. For instance, consider the two statements,

[11a] A cab was involved in an accident.

[11b] A witness identified a cab involved in an accident as a Blue cab.

In this case, although the intensional cue is present in statement [11b], it is not sufficient to convey the fact that the element defined as a cab in an accident in statement [11a] is the same as the element similarly described in statement [11b]. 
Yet, determinative cue are not always sufficient to guarantee the updating of an existing elementary representation. The presence of the determinative cue may be sufficient when personal pronouns are used to refer to a previously defined element, as in the following statements,

[12a] A cab was involved in an accident.

[12b] A witness identified it as a Blue cab.

Still, there are other cases where the presence of a determinative cue may not be sufficient to lead to the same conclusions. For example, this would be the case when the intensional cue is ambiguous, as in the following statements,

[13a] A cab was involved in an accident.

[13b] A witness identified the vehicle as Blue.

where statement [13b] may be interpreted as referring to the cab defined statement [13a] or to another vehicle involved in the accident.

Finally, it is possible that the partial matching of the intensions of an existing element suffice to produce the same effect, as in the following statements,

[14a] A cab was involved in an accident.

[14b] A witness identified this cab as Blue.

where, although the second statement does not mention the accident, the combination of the use of the demonstrative pronoun 'this' and the intension 'cab' in statement [14b] should suffice to warrant the updating of the intensions of the original element.

\section{Updating sample representations}

Set statements entail the representation of new samples (Proposition 1c). Such samples will either be included in, or represented outside the existing sample space,

Proposition 2c. Provided that the representation of a sample space $\mathscr{S}_{i}$ has been elicited by a previous set statement, the representation of a new set $S_{j+1}$ will be included in $\mathscr{S}_{i}$ or in $S_{i}$, a sample already included in $\mathscr{S}_{i}$, if the following conditions are satisfied:

(i) There is an intensional cue: The intensions of the reference set of $S_{j+1}$ either match the intensions of $\mathscr{S}_{i}$ or those of $S_{j}$, thereby indicating that the representation of $S_{i+1}$ is to be included in $\mathscr{S}_{i}$ or in $S_{j}$, respectively.

(ii) There is a numerical cue: The size of the reference set of $S_{j+1}$ is either known to be equal to the size of the sample within which it is to be included (absolute formats) or the size of $S_{j+1}$ can be made relative to the size of this sample (relative formats).

(iii) There is a determinative cue: A definite determiner is used to indicate that the elements of the reference set of $S_{j+1}$ are the same as the elements of the sample within which the representation of $S_{j+1}$ is to be included.

As for the representation elementary statements, the presence of the three cues should guarantee the inclusion of a new sample representation within an existing sample or sample space. In this case, however, both numerical and determinative cues would be 
necessary for inclusion. The absence of a numerical cue precludes the presence a determinative cue. For example, consider the following two statements,

[15a] 10 out of 100 women have breast cancer.

[15b] 90 out of *these* 100 women with breast cancer will get a positive mammography.

In this case, the presence of the pronoun 'these' in statement [15b] is not felicitous because it suggests that its reference set $\mathscr{S}_{2}=\{$ woman $\wedge$ breast_cancer $\}$ is indeed the subset $S_{1}=\{$ woman $\wedge$ breast_cancer $\}$ defined in statement [15a] while the difference in sizes of $S_{1}$ and $\mathscr{S}_{2}$ [i.e., $n\left(S_{1}\right)=10$ vs. $n\left(\mathscr{S}_{2}\right)=100$ ] contradicts this possibility.

The presence of the numerical cue alone, however, may not be sufficient to entail an inclusive representation. Consider, for example, the following two statements,

[16a] $10 \%$ of women have breast cancer.

[16b] $90 \%$ of women with breast cancer get a positive mammography.

Although both intensional cues and numerical cues are present, the reference set of the second statement need not be the same as the subset defined in the first statement.

It is possible, however, that the presence of both a numerical cue and a personal pronoun as a determinative cue may warrant inclusion. For example, consider

[17a] $10 \%$ of women have breast cancer.

[17b] $90 \%$ of them will get a positive mammography.

In this case, although there is no intensional cue in the second statement, the use of the personal pronoun 'them' unambiguously indicates that the reference set of statement [17b] is indeed the same set of elements as the subset defined in statement [17a]. Yet, when demonstrative pronouns are used, the absence of an intensional cue will render the relationship between sets defined in separate statements ambiguous. For example, consider the following statements,

[18a] $10 \%$ of women have breast cancer.

[18b] $90 \%$ of patients with breast cancer will get a positive mammography.

\section{Inclusive representations}

Thus, the inclusion of new samples in the existing sample space representation will be facilitated by the use of a unique set of intensions to refer to a sample. For instance, consider the following sets of statements,

[19a] $10 \%$ of women have breast cancer.

[19b] $80 \%$ of these women who have breast cancer will get a positive mammography.

Statement [19a] entails a representation identical to representation (9) (see p. 196). Taken independently, statement [19b] would be based on the sample space defined by $\mathscr{S}_{2}=\{$ woman $\wedge$ breast_cancer $\}$. Included in $\mathscr{S}_{2}$, would be sample $S_{2}$, defined by 
$S_{2}=\{$ woman $\wedge$ breast_cancer $\wedge$ positive_mammography $\}$. Finally, the representation will include the probability of $S_{2}$, relative to $\mathscr{S}_{2}$, that will be denoted as $p\left(S_{2} \mid \mathscr{S}_{2}\right)$. In short, the representation of statement [19b] can be formalised as follows,

$$
S_{2}=\left\{\mathscr{S}_{2}=\{\text { woman } \wedge \text { breast_cancer }\}\right.
$$

But given that statement [19b] follows statement [19a] and that (i) $S_{1}$ and $\mathscr{S}_{2}$ share the same intensions, namely woman $\wedge$ breast_cancer, and (ii) the size of $S_{2}$ can be made relative to the size of $S_{1}$ (i.e., $80 \%$ of $10 \%$ ), and finally, given that (iii) there is a determinative cue to indicate that the elements in $S_{1}$ are the elements of the reference set of statement [19b] (i.e., "those women with breast cancer"), it follows from Proposition 2c that the representation elicited by statement [19a] (see diagram (9), p. 196) can be updated as statement [19b] is processed. Specifically, $\mathscr{T}_{2}$ will be assimilated with $S_{1}$ and the new sample $S_{2}=\{$ woman $\wedge$ breast_cancer $\wedge$ positive_mammography $\}$ will be included in the existing sample $S_{1}=\{$ woman $\wedge$ breast_cancer $\}$. It follows that representation (19a) will be updated to result in the following representation,

$$
\begin{aligned}
& S_{1}=\{\text { woman }\wedge \text { breast_cancer }\} \\
& p\left(S_{1} \mid \mathscr{S}_{1}\right)=10 \% \\
& S_{2}=\{\text { woman } \wedge \text { breast_cancer }\wedge \text { mammography }\} \\
& p\left(S_{2} \mid S_{1}\right)=80 \%
\end{aligned}
$$

\section{Multiple sample representations}

If the conditions outlined in Proposition 2c are not respected, the updating of the representation will lead to multiple samples. For example, consider the following sets of statements.

[20a] 10 out of 100 women have breast cancer.

[20b] 80 out of 100 women with breast cancer will get a positive mammography.

In this case, the representation of the first statement will be similar to that of the representation (9) except that the absolute size of both $\mathscr{S}_{1}$ and $S_{1}$, denoted as $n\left(\mathscr{S}_{1}\right)$ and $n\left(S_{1}\right)$, respectively, will feature in the representation instead of the probability $p\left(S_{1} \mid \mathscr{S}_{1}\right)$. The representation of the second statement will be similar to that of the representation (19b), except again that the respective sizes of $\mathscr{S}_{2}$ and $S_{2}$ will feature in the representation instead of the probability of $p\left(S_{2} \mid \mathscr{S}_{2}\right)$. Sample $\mathscr{S}_{2}$ and sample $S_{1}$ have the same intension, thereby satisfying condition i of Proposition 2c. In this case, however, 
neither condition ii nor condition iii of this proposition is satisfied. Namely, $S_{1}$ and $\mathscr{T}_{2}$ differ in size and there is no determinative cue to indicate that $S_{1}$ actually is the reference set of $S_{2}$. Consequently, $S_{2}$ will not be included in $S_{1}$ and, taken together, statements [20a] and [20b] will entail the following representation,

$$
\begin{aligned}
& \begin{aligned}
& \mathscr{S}_{1}=\{\text { woman }\} \\
& n\left(\mathscr{S}_{1}\right)=100 \\
& S_{1}=\{\text { woman } \wedge \text { breast_cancer }\} \\
& n\left(\bar{S}_{1}\right)=10
\end{aligned} \\
& \mathscr{S}_{2}=\{\text { woman } \wedge \text { breast_cancer }\}
\end{aligned}
$$

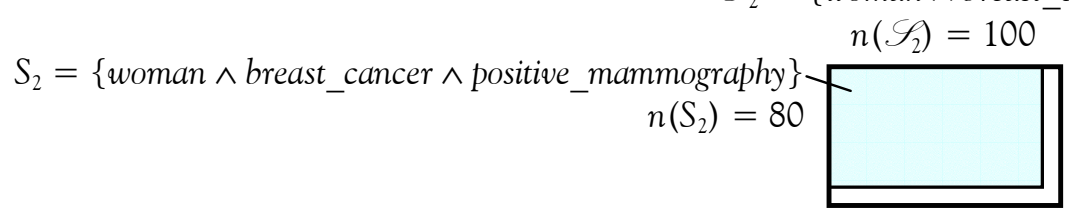

There is another situation that will not lead to the inclusion of $S_{2}$ in $S_{1}$. Consider the following sets of statements,

[21a] $85 \%$ of the cabs in the city are blue.

[21b] $80 \%$ of the blue cabs presented to a witness were correctly identified.

Although these two statements might appear to be equivalent to statements [19a] and [19b], respectively (see p. 200), SSR theory predicts that they would lead to a quite different mental representation. The representation of statement [21a] will be based on the sample space $\mathscr{S}_{1}$ defined by $\mathscr{S}_{1}=\left\{c i t y \_c a b\right\}$. Included in $\mathscr{S}_{1}$, will be the sample $S_{1}$, defined by $S_{1}=\{$ city_cab $\wedge$ blue $\}$. Finally, the representation will include the probability of $S_{1}$, relative to $\mathscr{S}_{1}$, denoted here as $p\left(S_{1} \mid \mathscr{S}_{1}\right)$,

$$
\begin{gathered}
\mathscr{S}_{1}=\left\{c i t y_{-} c a b\right\} \\
S_{1}=\left\{c i t y \_c a b \wedge b l u e\right\} \\
p\left(S_{1} \mid \mathscr{S}_{1}\right)=85 \%
\end{gathered}
$$

Statement [21b] will entail a representation based on the sample space $\mathscr{S}_{2}$ defined by $\mathscr{T}_{2}=\{$ presented_cab $\wedge$ blue $\}$. Included in $\mathscr{S}_{2}$, will be the sample $S_{2}$ defined by $S_{2}=$ $\{$ presented_cab $\wedge$ blue $\wedge$ correctly_identified\}. Finally, the representation will include the probability of $S_{2}$, relative to $\mathscr{S}_{2}$, denoted here as $p\left(S_{2} \mid \mathscr{S}_{2}\right)$, 


$$
\begin{array}{r}
\mathscr{S}_{2}=\{\text { presented_cab } \wedge \text { blue }\} \\
S_{2}=\{\text { presented_cab } \wedge \text { blue } \wedge \text { correctly_identified }\} \\
p\left(S_{2} \mid \mathscr{\mathscr { S }}_{2}\right)=80 \%
\end{array}
$$

Given that statement [21b] follows statement [21a], the initial representation will be updated. But, since $S_{1}$ and $\mathscr{S}_{2}$ do not have the same intensions (i.e., city_cab $\wedge$ blue vs. presented_cab $\wedge$ blue), the updated representation will be composed of two independent sample spaces, $\mathscr{T}_{1}$ and $\mathscr{S}_{2}$.

$$
\begin{array}{r}
S_{1}=\left\{c i t y \_c a b\right\} \\
p\left(S_{1} \mid \mathscr{S}_{1}\right)=85 \% \\
S_{2}=\{\text { presented_cab } \wedge \text { blue } \wedge \text { correctly_identified }\} \\
p\left(S_{2} \mid \mathscr{S}_{2}\right)=80 \%
\end{array}
$$

\section{Overview of updating representation stage}

SSR theory thus assumes that the second stage of processing entailed by Textbook and Set-based problems is the updating of the initial representation, which is again mediated by the type and format of the first statement presented in the task. Figure VII3 presents a flowchart summarising the processes potentially involved in the updating stage. Once all the task statements have been processed, SSR theory assumes that individuals will proceed to make their judgement. The processes at work in this final stage will be described in the next section. 


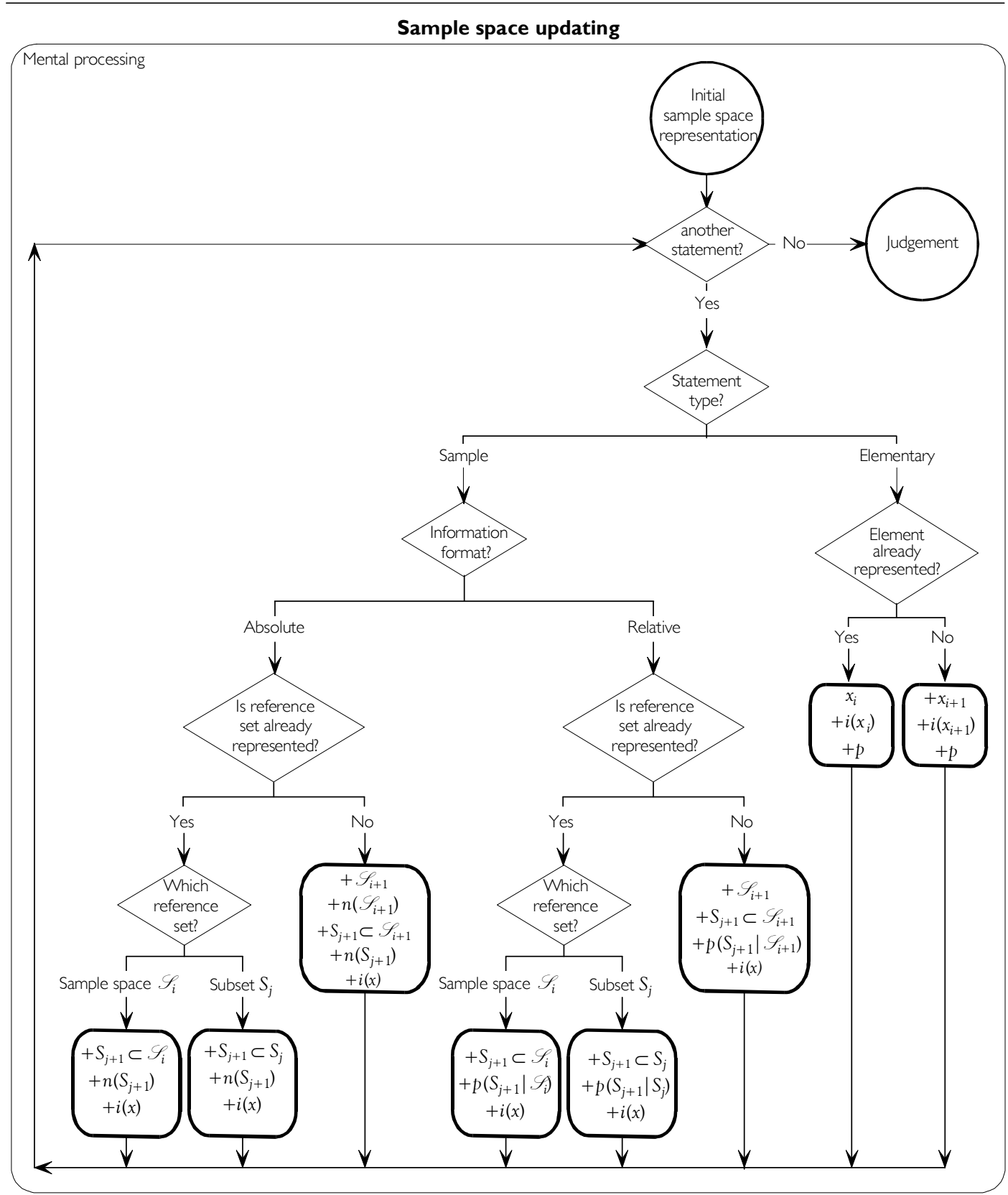

Figure VII-3 - Flow diagram summarising the processes involved in the updating of the initial representation of a posterior probability judgement task.

Note. The flowchart represents all the processes that are potentially at work in updating the representation of the task. The flow followed by a given individual processing the task is determined by the path followed at each decision node.

Altogether, the propositions of SSR theory lead to a first corollary, which defines the conditions for a fully inclusive representation of the statements of a posterior probability judgement task. Within the Textbook and Set-based paradigms, posterior probability judgement tasks usually involve three types of quantified statements: a statement for the base-rate information, a statement for the likelihood of observing the data, when the hypothesis is true and a statement for the likelihood of observing the data when the 
hypothesis is false. Corollary 1 delineates the conditions for a fully inclusive representation in such problems.

Corollary 1. A posterior probability judgement task will elicit a fully inclusive representation if:

(i) The base rate statement defines a reference set $\mathscr{S}$ and two exclusive and mutually exhaustive subsets, $H_{1}$ and $H_{0}$.

(ii) Intensional cue: The likelihood statements expressing $p\left(D \mid H_{1}\right)$ and $p\left(D \mid H_{0}\right)$ define reference sets whose intensions match the intensions of $H_{1}$ and $H_{0}$, respectively.

(iii) Numerical cue: The likelihood statements for $p\left(D \mid H_{1}\right)$ and $p\left(D \mid H_{0}\right)$ respectively define subsets $D \cap H_{1}$ and $D \cap H_{0}$ whose sizes can be made relative to the size of $H_{1}$ and $H_{0}$, respectively.

(iv) Determinative cue: The elements of the reference sets defined by the likelihood statements for $p\left(D \mid H_{1}\right)$ and $p\left(D \mid H_{0}\right)$ are indicated to be the elements of $H_{1}$ and $H_{0}$, respectively.

The crucial role of fully inclusive representations in eliciting Bayesian judgements will be demonstrated in the next section.

\section{Final representations and judgement}

The final representation will include all the quantified statements in a given task. Within the Textbook-problem paradigm, the base-rate information is usually presented in a relative frequency format (e.g., " $85 \%$ of the cabs in the city are Green", Tversky \& Kahneman, 1980, p. 62). Likelihoods are usually expressed with set statements in relative frequency formats related to instances (e.g., "when presented with a sample of cabs ... the witness made correct identifications in $80 \%$ of the cases", ibid.) or occurrences (e.g., "the witness was able to identify each color correctly about $80 \%$ of the time”, Bar-Hillel, 1980, p. 212). Sometimes, likelihoods can also be presented in elementary statements (e.g, "If someone is a sufferer, there is $90 \%$ chance that he will be recorded positively", Hammerton, 1973, p. 252). There is usually the mention of the presence of an elementary statement to indicate that a datum has been observed (e.g., "A witness identified the cab as a Blue cab", Tversky \& Kahneman, 1980, p. 62; "Mr. Smith has been tested, and the result is positive", Hammerton, 1973, p. 252).

The different possible combinations of statements' types and intensions are sizeable but not unlimited: each of the three quantified statements [i.e., $p(H), p(D \mid H)$, and $p(D \mid \sim H)$ statements] can be expressed in two different types (i.e., elementary or sample type), with either consistent or varying intensions, the presence or absence of a determinative cue, and a relative or absolute information format. Following from Proposition 1a, the final sample space representation of the task will be determined by the specific types and intensions of the statements that constitute the task. Similarly, the 
representation of the goal statement will be determined by its type and intensions. It was suggested earlier that the goal statement would elicit the application of a judgement method to the existing sample space representation, which will, in turn, result in the production of a judgement outcome. This assumption is formalised by the following proposition,

Proposition 3a. Individuals will review their representation of the task's premises and form their probability judgement on the basis of the samples or elements whose intensions match those of the goal statement to the highest degree.

If individuals always represent what is explicit in a given statement, Proposition $3 \mathrm{a}$ would lead to the prediction of a unique judgement output for any given task, a prediction which is at odds with the individual variability customarily observed. Whereas the origin of variability in judgements is often tacitly accounted for, SSR theory explicitly explains the origin of such variability. Namely, it is assumed that it originates from individual inferences leading to the modification of a representation that would otherwise solely be based on what is explicit in the statements of the task. This assumption is formalised by the next proposition (see p. 207 for an illustration),

Proposition 3b. Individuals may alter their representation of what is explicit in the statements, either to represent additional assumptions or to improve matching of the number of the samples or elements whose intensions match those of the goal statement.

\section{Judgement methods and elementary goal statements}

Depending on the type of the goal statement, SSR theory proposes that different judgmental methods will be applied. The following proposition defines the judgement method that will be elicited by elementary goal statements.

Proposition 3c. If the goal statement is elementary, individuals will review their representation and will base their judgement on the probability associated with the element or sample having the highest number of intensions matching those of the goal statement. If there is more than one element or sample thus identified, individuals will produce a judgement based on an aggregate of the probabilities associated with these elements and/or samples. The exact modalities of the aggregation (i.e., anchor and adjustment, multiplication, addition, etc.) will depend on individual abilities and preferences.

For example, consider a simple, generic, quantitative posterior probability task including a base-rate statement, two likelihood statements, a data statement and a goal statement, all in elementary form, 
[22a] The probability that $i_{1}(x)$ and $i_{2}(x)$ is $a \%$.

[22b] If $i_{1}(x)$ and $i_{2}(x)$, the probability that $i_{3}(x)$ is $b \%$.

[22c] If $i_{1}(x)$ and $\neg i_{2}(x)$, the probability that $i_{3}(x)$ is $c \%$.

[22d] There is $x_{0}$ such that $i_{1}\left(x_{0}\right)$ and $i_{3}\left(x_{0}\right)$.

[22e] What is the probability that $i_{2}\left(x_{0}\right)$ ?

Intension $i_{1}\left(x_{0}\right)$ is a basic intension defining the type of element considered such as ' $x_{0}$ is a person' or ' $x_{0}$ is a cab.' Usually, $i_{2}\left(x_{0}\right)$ is the intension of the hypothesis, such as ' $x_{0}$ has a disease' or ' $x_{0}$ is blue,' and $\neg i_{2}(x)$ is the negation of $i_{2}(x)$. Finally, $i_{3}\left(x_{0}\right)$ is the intension of the data, for example, ' $x_{0}$ has a symptom' or ' $x_{0}$ is correctly identified.' For example, a concrete version of the generic task above, where $i_{1}(x)=$ " $x$ is a person," $i_{2}(x)=$ " $x$ has flu," and $i_{3}(x)=$ " $x$ has a sore throat," would read,

[23a] The probability that a person has flu is $10 \%$.

[23b] If a person has flu, the probability that he has a sore throat is $55 \%$.

[23c] If a person does not have flu, the probability that he has a sore throat is $25 \%$.

[23d] A person has a sore throat.

[23e] What is the probability that he has flu?

According to Propositions $1 \mathrm{~b}$ and $2 \mathrm{a}$, these statements would be represented as follows,

$$
\begin{aligned}
& \text { _person } \wedge[f l u, 10 \%] \\
& \text { _ }, \text { person } \wedge \text { flu } \wedge \text { [sore_throat, 55\%] } \\
& \text {,person } \wedge \neg f l u \wedge \text { [sore_throat, 25\%] } \\
& \text { _ person } \wedge \text { sore_throat } \wedge[f l u, \sqcup \%]
\end{aligned}
$$

Proposition 3a stipulates that individuals will review the representation of the task to identify the model or models having the highest number of intensions matching those of the goal statement. In this example, the goal statement indicates that the element evaluated may be characterised by the intensions person $\wedge$ sore_throat $\wedge$ flu. Those who make this interpretation are likely to base their judgement solely on representation (23b), and answer with the value of $55 \%$. This answer corresponds to an inverse fallacy judgement, as described in Chapter III.

Thus, although SSR theory predicts that most individuals will commit the inverse fallacy in elementary tasks, it also allows other judgements to be observed when individuals update their representation to include new assumptions or to improve matching (Proposition 3b). In the present example, it is possible that some judges will assume that someone who has a sore throat may or may not have flu. In the SSR theoretical framework, this means that judges may infer that if an element is described 
by person $\wedge$ sore_throat, it may be described by person $\wedge$ sore_throat $\wedge$ flu or by person $\wedge$ sore throat $\wedge \neg f l u$. Such an interpretation would in turn lead to the following representation of the statements [23d] and [23e],

$$
\text { ¿ }
$$

where the symbol " $\vee$ " represents the logical connector "or". The intensions of this alternative representation of statements [23d] and [23e], denoted representation $\left(23^{*} \mathrm{~d}, \mathrm{e}\right)$ match the intensions of models $(23 \mathrm{~b})$ and $(23 \mathrm{c})$ to the same degree. As a result, SSR theory predicts that judgements based on this representation will entail an aggregation of the probabilities associated with each of these models, namely, 25\% and 55\% (Proposition 3c).

In the example above, the task involved both elementary premise statements and an elementary goal statement. Elementary goal statements can also be involved with tasks based on set statements. A generic task made of set statements would be of the form,

[24a] $a \%$ of $i_{1}(x)$ elements are $i_{2}(x)$ elements and the remaining $(100-a) \%$ are $\neg i_{2}(x)$ elements.

[24b] $b \%$ of these $i_{1}(x)$ and $i_{2}(x)$ elements, are $i_{3}(x)$ elements.

[24c] $c \%$ of the remaining $i_{1}(x)$ and $\neg i_{2}(x)$ elements, are also $i_{3}(x)$ elements.

where intensions $i_{1}(x), i_{2}(x)$ and $i_{3}(x)$, respectively, are similar to the intensions defined in the previous generic task (see p. 207), and $\neg i_{2}(x)$ is the negation of intension $i_{2}(x)$. For example, a concrete version of the generic task above could read,

[25a] 10\% of people have flu and the remaining $90 \%$ do not have flu.

[25b] $55 \%$ of those people who have flu have a sore throat.

[25c] 25\% of the remaining people who do not have flu also have a sore throat.

Following Proposition 1c, and since both the statements of the task respect the conditions for a fully inclusive representation (Corollary 1), the representation of this task can be formalised as follows,

$$
\begin{gathered}
S_{1}=\{\text { person } \wedge \text { flu }\} \\
p\left(S_{1} \mid \mathscr{S}_{1}\right)=10 \% \\
S_{3}=\{\text { person } \wedge \text { flu } \wedge \text { sore throat }\} \\
p\left(S_{3} \mid S_{1}\right)=55 \% \\
S_{2}=\{\text { person } \wedge \neg f l u\} \\
p\left(S_{2} \mid \mathscr{S}_{1}\right)=90 \% \\
S_{4}=\{\text { person } \wedge \neg f l u \wedge \text { sore throat }\} \\
p\left(S_{4} \mid S_{2}\right)=25 \%
\end{gathered}
$$

A generic elementary goal statement would be as follows, 
[24d] There is $x_{0}$ such that $i_{1}\left(x_{0}\right)$ and $i_{3}\left(x_{0}\right)$.

[24e] What is the probability that $i_{2}\left(x_{0}\right)$ ?

For example, in the flu scenario, this would give,

[25d] A person has a sore throat.

[25e] What is the probability that this person has flu?

and would be represented as,

$$
\text { _ person } \wedge \text { sore_throat } \wedge[f l u, \sqcup \%]
$$

According to Proposition 3c, individuals would then review their representation, identify the sample whose intension matches that of the goal statement (namely, person $\wedge$ sore_throat $\wedge f(u)$, and base their judgement on the probability associated with the identified sample. In the present case, there is only one sample whose intension matches that of the goal statement, namely sample $S_{3}$. So, representation (25d) would lead individuals to solely base their judgement on $p\left(S_{3} \mid S_{1}\right)$, which again corresponds to an inverse fallacy judgement.

Alternatively, statements [25d] and [25e] may entail the following representation,

$$
\text { , person } \wedge \text { sore_throat } \wedge[f l u \vee \neg f l u, \sqcup \%]
$$

in which case, SSR theory then predicts that individuals will combine both $p\left(S_{3} \mid S_{1}\right)$ and $p\left(S_{4} \mid S_{2}\right)$ as the intensions of $S_{3}$ and $S_{4}$ both match those of the goal statement to the same extent (Proposition 3c).

In sum, SSR theory explains why, in tasks where the goal statement is presented in an elementary form, most individuals will commit the inverse fallacy; that is, they will base their judgement solely on the value of $p(D \mid H)$. The origin of this explanation is twofold. Firstly, it follows from the assumption that elementary goal statements will lead individuals to search their representation for models with the highest number of intensions matching those of the goal statement (Proposition 3c). Secondly, it follows from the fact that the representation of what is explicit in the statements of a posterior probability task will always result in the representation of the $p(D \mid H)$ statement having the highest number of intensions matching those of the goal statement.

SSR theory also accounts for the fact that not all individuals will exhibit this fallacy by explaining individual differences in the judgements observed, and proposes that among those individuals who do not commit the inverse fallacy, some will combine the probabilities of $p(D \mid H)$ and $p(D \mid \sim H)$ when they assume that an element of $D$ may or may not belong to $H$. This explanation is consistent with the so-called "base-rate 
neglect" phenomenon. Namely, most individuals will not readily rely on the probability associated with the base-rate information in their judgements because the intensions of the likelihood probability statements match those of the goal statement more closely than the intensions of the base-rate statement.

\section{Judgement methods and set goal statements}

SSR theory predicts that quite different judgement processes will be involved following the provision of a set goal statement. Specifically, the judgement method elicited by set goal statements is summarised by Propositions 3d and 3e,

Proposition $3 \mathrm{~d}$. If the goal statement is a set statement, individuals will evaluate the proportion of elements in the sample(s) ${ }^{1}$ whose intension(s) match those of the subset defined in the goal statement relative to the proportion of elements in the sample(s) whose intension(s) match those of the reference set of the goal statement.

Proposition 3e. If the proportions highlighted by the set goal statement are relative to different samples within a unique sample space, individuals will first relate these proportions to this sample space. If those proportions are relative to different sample spaces, individuals will either assimilate (i.e., consider that $\mathscr{S}=\mathscr{S}_{\mathrm{i}}=\mathscr{S}_{\mathrm{j}}$ where $\mathscr{S}_{\mathrm{i}}$ and $\mathscr{S}_{\mathrm{j}}$ are the sample spaces that include the samples highlighted by the goal statement) or combine the different sample spaces (i.e., consider that $\mathscr{S}=\mathscr{S}_{\mathrm{i}} \cup \mathscr{S}_{\mathrm{j}}$ ).

Consider the following generic task statement,

[24f] Among those elements that are $i_{1}$ and $i_{3}$, what is the percentage of $i_{2}$ elements?

This gives, in the flu example,

[25f] Among those people who have a sore throat, what is the percentage of people who have flu?

According to Proposition 3d, statement [24f] will lead individuals to evaluate the proportions of elements in the sample described by intensions 'person,', 'sore_throat,' and 'flu' relative to the proportion of elements in the samples described by 'person' and 'sore_throat'. The full representation of the Set-based flu task including the following statements,

[25a] 10\% of people have flu and the remaining $90 \%$ do not have flu.

[25b] $55 \%$ of those people who have flu have a sore throat.

[25c] $25 \%$ of the remaining people who do not have flu also have a sore throat.

[25f] Among those people who have a sore throat, what is the percentage of people who have flu?

can thus be formalised as follows,

\footnotetext{
${ }^{1}$ Assuming that set goal statements will always be presented in conjunction with sample premise statements as it would make little sense to give information in elementary statements followed by a set goal statement.
} 


$$
\begin{gathered}
\mathscr{S}_{1}=\{\text { person }\} \\
p\left(S_{1} \mid \mathscr{S}_{1}\right)=10 \% \\
S_{3}=\{\text { person } \wedge \text { flu } \wedge \text { sore_throat }\} \\
p\left(S_{3} \mid S_{1}\right)=55 \% \\
S_{2}=\{\text { person } \wedge \neg f l u\} \\
p\left(S_{2} \mid S_{1}\right)=90 \% \\
S_{4}=\{\text { person } \wedge \neg f l u \wedge \text { sore_throat }\} \\
p\left(S_{4} \mid S_{2}\right)=25 \%
\end{gathered}
$$

$$
\begin{gathered}
\text { \{person } \wedge \text { sore_throat }\}=\mathscr{S} \\
\text { \{person } \wedge \text { sore_throat } \wedge \text { flu }\}=S \\
p(S \mid \mathscr{S})=\sqcup \%
\end{gathered}
$$

where the goal statement defines reference set $\mathscr{S}=$ \{person $\wedge$ sore throat $\}$ and the subset $S=\{$ person $\wedge$ sore_throat $\wedge f l u\}$ and call for the percentage of $S$ relative to $\mathscr{S}$.

Both $S_{3}$ and $S_{4}$ encompass elements described by intension 'person' and intension 'sore_throat', which can lead judges to conclude that $\mathscr{S}=S_{3} \cup S_{4}$. Similarly, sample $S=$ \{person $\wedge$ sore_throat $\wedge f l u$ \} can be identified with $S_{3}$ which is the only sample in the existing representation containing elements described by intensions 'person,' 'sore_throat' and 'flu.' Consequently, judges will be able to infer that the goal of the task is to evaluate $p\left(S_{3} \mid S_{3} \cup S_{4}\right)$. The representation of the goal of the task thus becomes,

$$
\begin{gathered}
\{\text { person } \wedge \text { sore throat }\}=\mathscr{S} \\
\therefore \mathscr{S}=\bar{S}_{3} \cup S_{4} \\
\{\text { person } \wedge \text { sore_throat } \wedge \text { flu }\}=S \\
\therefore \bar{S}=S_{3} \\
\therefore p\left(S \mid \mathscr{S}=p\left(S_{3} \mid S_{3} \cup S_{4}\right)=\sqcup \%\right.
\end{gathered}
$$

The proportions associated with $S_{3}$ and $S_{4}$, however, have different referents. Indeed $p\left(S_{3} \mid S_{1}\right)$ is relative to $S_{1}$ whereas $p\left(S_{4} \mid S_{2}\right)$ is relative to $S_{2}$. SSR theory predicts that individuals will first relate these quantities to a common sample space (Proposition 3e). Given that both $S_{3}$ and $S_{4}$ are included in sample space $\mathscr{S}_{1}$, individuals will be encouraged to evaluate both $p\left(S_{3} \mid \mathscr{S}_{1}\right)$ and $p\left(S_{4} \mid \mathscr{S}_{1}\right)$. To this end, they may infer that if $10 \%$ of people in $\mathscr{S}_{1}=\{$ person $\}$ belong to $S_{1}=\{$ person $\wedge f l u\}$, and $55 \%$ of these people belong to $S_{3}=$ pperson $\wedge$ flu $\wedge$ sore_throat , then $55 \%$ of $10 \%$ of the elements in $S_{3}$ belong to $\mathscr{S}_{1}$. Consequently, to evaluate $p\left(S_{3}\right)$ in relation to $\mathscr{S}_{1}$ is to evaluate $p\left(S_{3} \mid \mathscr{S}_{1}\right)=$ $55 \% \times 10 \% \approx 6 \%$. Alternatively, individuals may use an arbitrary reference number for $n\left(\mathscr{S}_{1}\right)$ and evaluate the relative number of elements in $S_{1}$ [e.g., $n\left(\mathscr{S}_{1}\right)=100, n\left(\mathscr{S}_{1}\right) \times$ $10 \%=n\left(S_{1}\right)=10$ ] and the relative number of elements in $S_{3}$ [i.e., $n\left(S_{1}\right) \times 55 \%=10 \times$ 
$\left.55 \% \approx 6=n\left(S_{3}\right)\right]$. Similar reasoning may lead them to evaluate the proportion of elements in $S_{4}$ relative to the sample space $\mathscr{S}_{1}$ [i.e., by evaluating $p\left(S_{4} \mid \mathscr{S}_{1}\right)=90 \% \times$ $25 \% \approx 23 \%$ or by evaluating $\left.n\left(S_{4}\right)=[100 \times 90 \%] \times 25 \% \approx 23\right)$. Finally, individuals will be able to form their judgement by evaluating either the ratio of $p\left(S_{3} \mid \mathscr{S}_{1}\right)$ to the sum of $p\left(S_{3} \mid \mathscr{S}_{1}\right)$ and $p\left(S_{4} \mid \mathscr{S}_{1}\right)$ [i.e., by evaluating $6 \%$ of $(6+23) 29 \%$ ] or by evaluating the ratio of $n\left(S_{3}\right)$ to the sum of $n\left(S_{3}\right)$ and $n\left(S_{4}\right)$ [i.e., by evaluating the ratio $6 \div 29 \times 100$ ].

Thus, contrary to tasks where the goal statement is an elementary statement, a set goal statement would allow for Bayesian judgements to be elicited, provided that individuals appropriately relate the relevant probabilities to the total sample space. Yet, not all sample tasks would allow Bayesian judgements to be elicited. Consider a task based on set statements that would not respect the conditions for a fully inclusive representation as defined in Corollary 1 (see p. 205). For instance, consider the following statements,

[26a] $a \%$ of $i_{1}$ elements are $i_{2}$ elements, [26b] $b \%$ of $i_{3}$ and $i_{2}$ elements, are $i_{4}$ elements, [26c] $c \%$ of $i_{3}$ and $i_{5}$ elements, are $i_{4}$ elements, [26d] Among $i_{3}$ and $i_{4}$ elements, what is the percentage of $i_{2}$ elements?

Consider, for instance, this version of the flu problem,

[27a] $10 \%$ of people have flu.

[27b] $55 \%$ of patients diagnosed with flu have a sore throat,

[27c] $25 \%$ of healthy patients have a sore throat,

[27d] Among patients who have a sore throat, what is the percentage of patients with flu?

The conditions laid out in Corollary 1 are not respected in these statements. In particular, there are neither intensional cues nor determinative cues to indicate that the samples defined in statements [27b] and [27c] should be included in the sample space defined by statement [27a]. Consequently, each of these statements will lead to the representation of a different sample space. The representation of this task can thus be formalised as follows: 


$$
\begin{aligned}
& \mathscr{S}_{1}=\{\text { individual }\} \\
& \begin{array}{l}
S_{1}=\{\text { individual } \wedge f l u\} \\
p\left(S_{1} \mid \mathscr{S}_{1}\right)=10 \%
\end{array} \\
& \begin{array}{l}
\mathscr{S}_{2}=\{\text { patient } \wedge f l u\} \\
\qquad \begin{array}{l}
S_{2}=\{\text { patient } \wedge \text { flu } \wedge \text { sore_throat }\} \\
p\left(S_{2} \mid \mathscr{S}_{2}\right)=55 \%
\end{array}
\end{array} \\
& \mathscr{S}_{3}=\{\text { patient } \wedge \text { healthy }\} \\
& \begin{array}{|l|l|}
\hline \\
\hline
\end{array} \\
& \mathrm{S}_{3}=\{\text { patient } \wedge \text { healthy } \wedge \text { sore_throat }\} \\
& p\left(S_{3} \mid \mathscr{S}_{3}\right)=25 \% \\
& \text { \{patient } \wedge \text { sore throat }\}=\mathscr{S} \\
& \therefore \mathscr{S}=\bar{S}_{2} \cup S_{3} \\
& \text { \{patient } \wedge \text { sore_throat } \wedge \text { flu }\}=S \\
& \therefore S=S_{2} \\
& \therefore p\left(S \mid \mathscr{S}=p\left(S_{2} \mid S_{2} \cup S_{3}\right)=\sqcup \%\right.
\end{aligned}
$$

The set goal statement calls for the evaluation of the proportion of elements in $S=$ \{patient $\wedge$ sore_throat $\wedge f l u\}$ relative to the elements in $\mathscr{S}=\{$ patient $\wedge$ sore_throat $\}$. From the representation of the task, judges can infer that $\mathscr{S}=S_{2} \cup S_{3}$ and that $S=S_{2}$, and therefore conclude that the goal of the task is to evaluate $p\left(S_{2} \mid S_{2} \cup S_{3}\right)$. But, since these samples are represented in different sample spaces (i.e., $\mathscr{S}_{2}$ and $\mathscr{S}_{3}$, respectively), Proposition $3 \mathrm{~d}$ suggests that judges will first relate $S_{2}$ and $S_{3}$ to a common sample space. Still according to this proposition, there are two ways in which they can achieve this, either by assimilating or by combining $\mathscr{S}_{2}$ and $\mathscr{S}_{3}$. The assimilation would consist in considering that $\mathscr{S}=\mathscr{S}_{1}=\mathscr{S}_{2}$. The combination would consist in considering that $\mathscr{S}=$ $\mathscr{S}_{1} \cup \mathscr{S}_{2}$

In any case, the resulting judgement will not match the Bayesian criterion and the base-rate information [i.e., $p\left(S_{1} \mid \mathscr{S}_{1}\right)$ ] will be ignored. Depending on whether individuals assimilate or combine $\mathscr{S}_{2}$ and $\mathscr{S}_{3}$, their final judgement will be based on one of the following two representations, 


$$
\begin{aligned}
& \text { Assimilation } \\
& \mathscr{S}=\{\text { patient }\}=\mathscr{S}_{1}=\mathscr{S}_{2}
\end{aligned}
$$

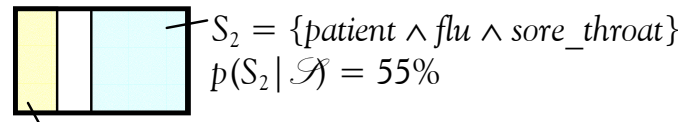

$$
\begin{aligned}
& \mathrm{S}_{3}=\{\text { patient } \wedge \text { healthy } \wedge \text { sore throat }\} \\
& p\left(S_{3} \mid \mathscr{S}\right)=25 \% \\
& \mathscr{S}=\{\text { patient }\}=\mathscr{S}_{1} \cup \mathscr{S}_{2}
\end{aligned}
$$

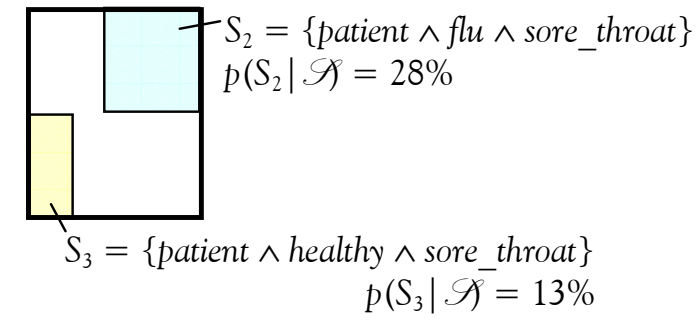

It is worth noting that if individuals first 'convert' the probability information in an absolute format (e.g., by arbitrarily assigning a number to $\mathscr{S}_{1}$ and $\mathscr{S}_{2}$ and inferring $n\left(\mathrm{~S}_{2}\right)$ and $n\left(S_{3}\right)$ in $\mathscr{S}$, the subsequent evaluation of the number of elements in $S_{2}$ relative to the number of elements in $S_{2}$ and $S_{3}$ would be independent of whether individuals combine or assimilate sample spaces $\mathscr{S}_{1}$ and $\mathscr{S}_{2}$.

\section{Overview of judgement stage}

This section thus delineated the processes underlying judgements based on the representation of the statements presented in either Textbook and Set-based posterior probability judgement tasks. These processes are assumed to form the last stage involved in such tasks, thus leading to the observation of the judgement output. Figure VII-4 presents a flowchart summarising the processes involved in this ultimate stage. 


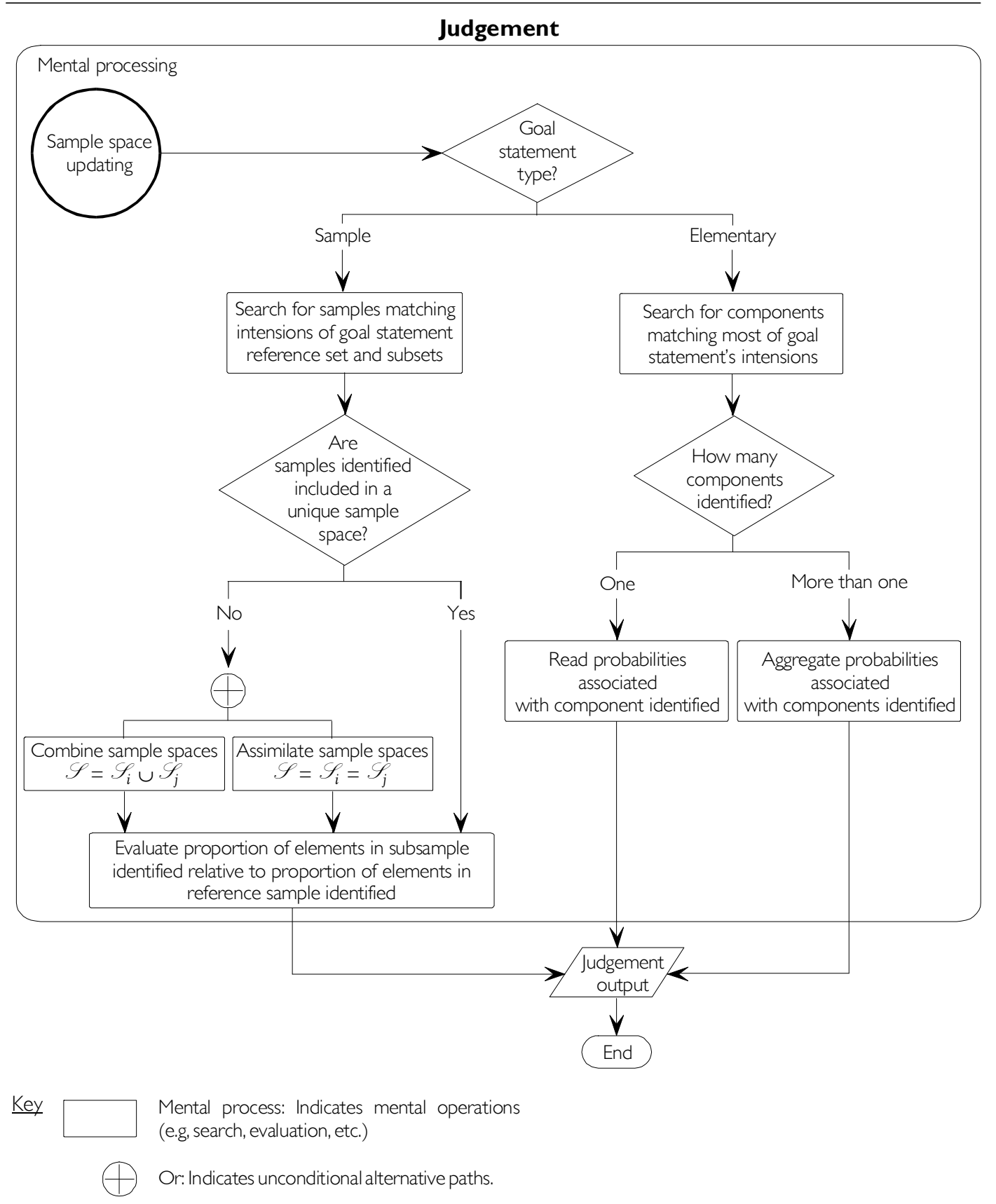

Figure VII-4 - Flow diagram summarising the processes underlying the final judgement output in posterior probability judgement tasks.

Altogether, these results lead to the second corollary of SSR theory, namely,

Corollary 2. Participants' posterior probability judgement of $p(H \mid D)$ will be Bayesian if,

(i) The intensions of the task statements lead to a fully inclusive representation (see Corollary 1, p. 205).

(ii) The goal statement is a set statement.

(iii) The intension(s) of the reference set defined in the goal statement can be matched to the intension(s) of the sample $D$ whose representation was elicited by the likelihood statements of the task (intensional cue) and the elements of this reference set are indicated to be the elements of $D$ (determinative cue).

(iv) The intension(s) of the subset defined in the goal statement can be matched to the intension(s) of the sample $\mathrm{H}$ elicited by the base-rate statement of the task 
(intensional cue) and the elements of this subset are indicated to be the elements of $H$ (determinative cue).

(v) Individuals can calculate the size of $H$ relative to the size of sample $D$ (numerical cue).

It follows that participants will not naturally provide a Bayesian judgement if either the task statements or the task goal statement are elementary statements (conditions $\mathrm{i}$ and ii, respectively). Interestingly, this account shows that the elicitation of Bayesian judgements is not limited to tasks framed in discrete formats (i.e., natural frequency or partitioned chance formats); it also allows for Bayesian judgements to be elicited using relative frequency formats. This account also indicates that the format is not sufficient to elicit correct judgements. If the conditions for inclusion are not satisfied or if the representation of the task is not fully inclusive (as defined in Corollary 1), the judgement method will lead to an evaluation that differs from the Bayesian criterion. It has already been shown that the removal of the numerical cue (Proposition 2c, condition ii) dramatically decreases the proportion of Bayesian judgements observed in sample tasks using a discrete format (e.g., see Girotto \& Gonzalez, 2001). According to SSR theory, however, this is not the only cue that may impede the elicitation of Bayesian answers. The last example indeed suggested that the absence of both intensional and determinative cues may have the same detrimental effects, by preventing a fully inclusive representation of the task statements. This illustrates how new testable empirical predictions can be derived from the propositions of SSR theory.

Conditions iii and iv of the present Corollary demonstrate the importance of the intensions used to define the reference set and subset of the goal statement. Differing intensions to refer to the elements already represented may undermine the search for the relevant samples when the judgement is made. Finally, Corollary 2 also demonstrates the importance of rudimentary computational knowledge, especially with regards to set statements expressed in a relative frequency format. Namely, in order to be able to calculate the size of $H$ relative to the size of sample $D$ whose representation was elicited by the task likelihood statements (Corollary 2, condition v), individuals will have to either (1) know that $x \%$ of $y \%$ is equal to $x \times y \div 100$ and $x \%$ out of $y \%$ is equal to $x \div y \times 100$, or (2) infer $n_{1}$ and $n_{2}$ from $x \times n \div 100$ and $y \times n \div 100$, respectively and calculate $\left(n_{1} \div n_{2} \times 100\right) \%$, where $x, y \in[0,100]$ and $n, n_{1}$, and $n_{2}$ are positive integers, or (3) to infer $n_{1}$ and $n_{2}$ directly from the representation elicited by the task and calculate $\left(n_{1} \div n_{2} \times 100\right) \%$, or else (4) to be asked to infer simply $n_{1}$ and $n_{2}$ directly from the representation elicited by the task. This last alternative, which is the less demanding 
from a computational point of view, corresponds to tasks framed in a discrete numerical format (i.e., either absolute frequencies or absolute chances).

Altogether, the principles of the SSR theory explain the processes underlying judgements of posterior probability observed in the Textbook paradigm and in the Setbased paradigm. In the following section of this chapter, I will briefly illustrate how the SSR theory both addresses issues previously raised in the review of the existing literature (see chap. III) while accounting for a wide range of existing results reported in the literature.

\author{
IMPLICATIONS OF SSR THEORY FOR THE EXISTING \\ LITERATURE ON POSTERIOR PROBABILITY JUDGEMENTS
}

\title{
On the causality account (Tversky \& Kahneman, 1980)
}

\section{The inverse fallacy and the taxicab problem}

Earlier, we saw that interpretational accounts have been advanced to account for the base-rate neglect effect. For example, Tversky and Kahneman (1980) proposed that the extent to which base-rate information fitted in a causal schema would determine whether it would be integrated in the final judgement. To test their hypothesis, they compared the standard cab problem with a "causal" scenario.

The standard cab problem (as reported in Tversky \& Kahneman, 1980, p. 290) consists of the following sets of statements:

[28a] A cab was involved in a hit-and-run accident at night.

[28b] $85 \%$ of the cabs in the city are Green, and $15 \%$ are Blue.

[28c] A witness identified the cab as a Blue cab.

[28d] When presented with a sample of cabs (half of which were Blue and half of which were Green) the witness made correct identifications in $80 \%$ of the cases and erred in $20 \%$ of the cases.

[28e] What is the probability that the cab involved in this accident was Blue rather than Green?

The authors found that 45\% of participants answered ' $80 \%$ ' when asked to complete this task (Tversky \& Kahneman, 1980).

The propositions of SSR theory can explain how the task led individuals to give this answer. Statement [28a] refers to a singular element (i.e., the cab involved in the accident). This statement will thus be represented by a unitary model, associated with intension $i_{1}(x)$ defined as $i_{1}(x)=$ " $x$ is a cab" and intension $i_{2}(x)$ defined as $i_{2}(x)=$ " $x$ is involved in an accident," 
$0^{\prime} c a b \wedge$ in_accident

Statement [28b] will entail a representation based on the sample space $\mathscr{S}_{1}$ defined by $\mathscr{S}_{1}$ $=\{$ city_cab $\}$. Included in $\mathscr{S}_{1}$ are the samples $S_{1}$ and $S_{2} . S_{1}$ is defined by $\left\{c i t y \_c a b \wedge\right.$ green $\}$ and $S_{2}$ is defined by $\left\{c i t y \_c a b \wedge b l u e\right\}$. Finally, the representation of statement [28b] will include the probabilities of $S_{1}$ and $S_{2}$ relative to $\mathscr{S}_{1}$, denoted $p\left(S_{1} \mid \mathscr{S}_{1}\right)$ and $p\left(S_{2} \mid \mathscr{S}_{1}\right)$, respectively. The representation of statement [28b] may be graphically characterised as follows,

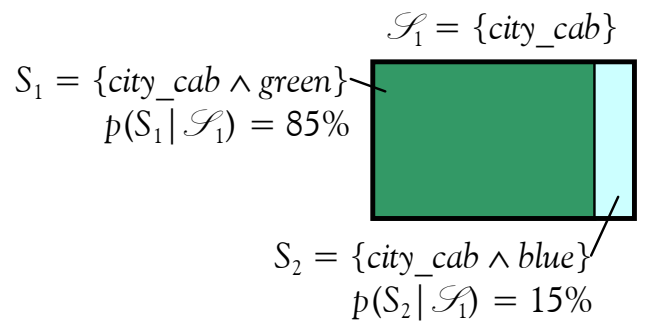

Statement [28c] refers to the same singular element as already represented in [28a] (i.e., the cab involved in the accident) so the process of this statement will result in the update of representation (28a) with the intension "identified_blue" (Proposition 2b),

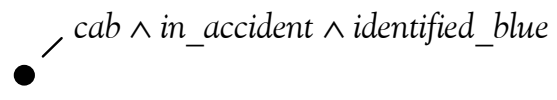

Statement [28d] refers to the set of cabs that are presented to the witness. Its representation will thus be based on sample $\mathscr{S}_{2}=\{$ presented_cab $\}$. It is said that half the sample is made of blue cabs and half is made of green cabs. Thus $\mathscr{L}_{2}$ includes two samples, namely $S_{3}=\{$ presented_cab $\wedge$ blue $\}$ and $S_{4}=\{$ presented_cab $\wedge$ green $\}$. It is also said that the witness made correct identifications in $80 \%$ of the cases and erred in $20 \%$ of the cases. Thus, the representation of $\mathscr{S}_{2}$ will also include samples $S_{5}=$ $\left\{\right.$ presented_cab $\wedge$ blue $\wedge$ correctly_identified\} and $S_{6}=$ \{presented_cab $\wedge$ blue $\wedge$ incorrectly_identified\}, as well as samples $S_{7}=$ \{presented_cab $\wedge$ green $\wedge$ correctly_identified $\}$ and $S_{8}=\{$ presented_cab $\wedge$ green $\wedge$ incorrectly_identified $\}$.

Additionally, the representation will feature the probabilities associated with these samples, in reference to the sample $\mathscr{S}_{2}$. The representation of statement [28d] may therefore be graphically characterised as follows, 


$$
\begin{aligned}
& \begin{array}{c}
S_{3}=\{\text { presented_cab } \wedge \text { blue }\} \\
p\left(S_{3} \mid \mathscr{S}_{2}\right)=50 \%
\end{array} \quad \mathscr{S}_{2}=\{\text { presented_cab }\} \\
& S_{4}=\{\text { presented } c a b \wedge \text { green }\} \\
& p\left(S_{4} \mid \mathscr{S}_{2}\right)=50 \% \\
& S_{7}=\{\text { presented_cab } \wedge \text { green } \wedge \text { correctly_identified }\} \\
& p\left(S_{7} \mid S_{4}\right)=80 \% \\
& \mathrm{~S}_{8}=\{\text { presented_cab } \wedge \text { green } \wedge \text { incorrectly_identified }\} \\
& p\left(S_{8} \mid S_{4}\right)=20 \% \\
& S_{5}=\{\text { presented_cab } \wedge \text { blue } \wedge \text { correctly_identified }\} \\
& p\left(S_{5} \overline{\mid} S_{3}\right)=80 \% \\
& \mathrm{~S}_{6}=\{\text { presented_cab } \wedge \text { blue } \wedge \text { incorrectly_identified }\} \\
& p\left(S_{6} \mid \bar{S}_{3}\right)=20 \%
\end{aligned}
$$

The goal statement, statement [28e], refers to the singular element introduced by statement [28a], and ask for the probability that this element can also be characterised by intension "blue." The complete representation of the task can therefore be formalised as follows,

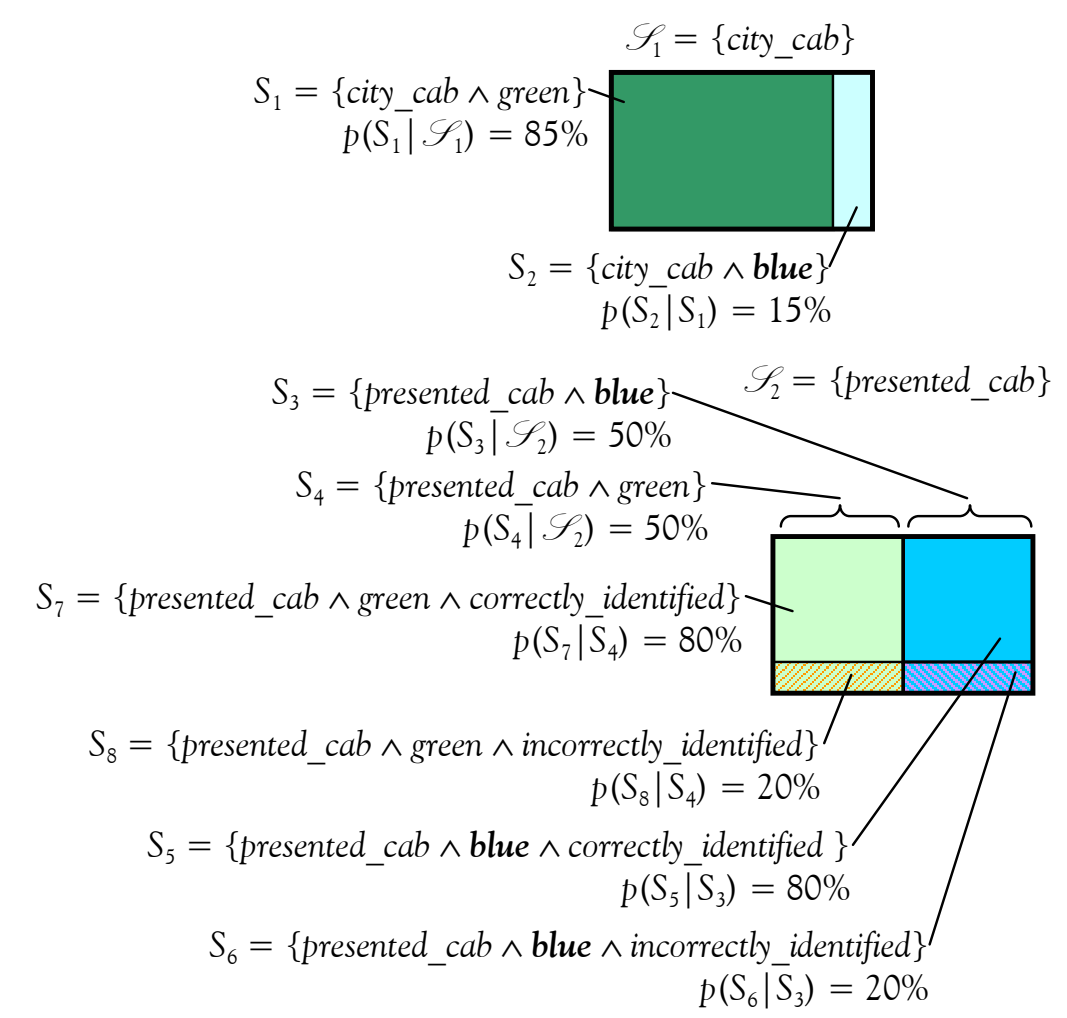

, $c a b \wedge$ in_accident $\wedge$ identified_blue $\wedge[$ blue, $\sqcup \%]$

$(28 \mathrm{a}, \mathrm{c}, \mathrm{e})$

Here, the goal statement has an elementary form, but the representation of the task is based on sample models. According to SSR theory, individuals will review their representation of the task's statements and base their judgement on the probability associated with the sample or model having the highest number of intensions matching 
those of the goal statement (Proposition 3c). The representation outlined above illustrates the complexity of the cab problem: the majority of samples defined by the task (namely, samples $S_{2}, S_{3}, S_{5}$, and $S_{6}$ ) have one intension in common with the goal statement (namely, intension "blue"). Although individuals may attempt to aggregate the probability values associated with these samples, there is an inference readily available that would improve matching. Individuals may indeed infer that the conjunction of intensions "identified_blue" and "blue" is equivalent to the conjunction of intensions "correctly_identified" and "blue" and update the representation of the goal statement accordingly, (Proposition 3b)

$$
\begin{aligned}
& \begin{array}{c}
\mathscr{S}_{1}=\left\{c i t y \_c a b\right\} \\
S_{1}=\left\{c i t y \_c a b \wedge g r e e n\right\} \\
p\left(S_{1} \mid \mathscr{S}_{1}\right)=85 \%
\end{array} \\
& S_{3}=\{\text { presented_cab } \wedge \text { blue }\} \quad \mathscr{S}_{2}=\{\text { presented_cab }\} \\
& p\left(S_{3} \mid \mathscr{S}_{2}\right)=50 \% \\
& \mathrm{~S}_{4}=\{\text { presented_cab } \wedge \text { green }\} \\
& p\left(\mathrm{~S}_{4} \mid \mathscr{S}_{2}\right)=50 \% \\
& S_{7}=\{\text { presented_cab } \wedge \text { green } \wedge \text { correctly_identified }\} \\
& p\left(S_{7} \mid \bar{S}_{4}\right)=80 \% \\
& S_{8}=\{\text { presented_cab } \wedge \text { green } \wedge \text { incorrectly_identified }\} \\
& p\left(\mathrm{~S}_{8} \mid \bar{S}_{4}\right)=20 \% \\
& \mathrm{~S}_{5}=\{\text { presented_cab } \wedge \text { blue } \wedge \text { correctly_identified }\} \\
& p\left(S_{5} \mid S_{3}\right)=80 \% \\
& \mathrm{~S}_{6}=\{\text { presented_cab } \wedge \text { blue } \wedge \text { incorrectly_identified }\} \\
& p\left(\mathrm{~S}_{6} \mid \bar{S}_{3}\right)=20 \% \\
& , c a b \wedge \text { in_accident } \wedge \text { identified_blue } \wedge[\text { blue, } \sqcup \%] \\
& \text { (28a, c, e) } \\
& \therefore \\
& \iota^{\prime c a b} \wedge \text { in_accident } \wedge \text { correctly_identified } \wedge[\text { blue }, \sqcup \%] \\
& \left(28^{*} \mathrm{a}, \mathrm{c}, \mathrm{e}\right)
\end{aligned}
$$

As a result, sample $S_{5}$ can be identified as the only sample having two intensions matching those of the goal statement, namely, intensions "correctly_identified" and "blue." Accordingly, SSR theory would predict that individuals will judge the posterior probability to be equal to the probability associated with this sample, namely $80 \%$. Thus, SSR theory provides an algorithmic account of the judgement outputs customarily observed in the cab problem (Tversky \& Kahneman, 1980). 


\section{Accounting for the 'causal effect'}

To test the causality account, according to which the base-rate information would no longer be neglected if it were included in a causal schema, Tversky and Kahneman (1980) modified their original scenario by replacing statement [28b] ("85\% of the cabs in the city are Green and 15\% are Blue") by the following statement:

[28b'] 85\% of cab accidents in the city involve Green cabs, and 15\% involve Blue cabs.

The authors reported that this modification led only $18 \%$ of participants to rely on the inverse likelihood (.80) to make their judgements, compared to $45 \%$ with the original task. The authors also noted that another $18 \%$ of participants answered with the baserate information (.15) compared to only $7 \%$ in the original task whereas the majority of participants (60\%) exhibited 'intermediate' judgement outputs, compared with 35\% in the original task. Tversky and Kahneman (1980) argued that this effect was due to the fact that the latter formulation of the base-rate information elicited "a causal explanation which makes any particular Green cab more likely to be involved in an accident than any particular Blue cab.” (Tversky \& Kahneman, 1980, p. 65). It was contended here (see chap. III) that this explanation did not constitute an algorithmic account of judgements observed as the ways in which such a causal interpretation would determine judgements observed (e.g., exclusive reliance of base-rate information or integration of different probability quantities) was left unspecified.

By contrast, the propositions of SSR theory, which characterise individuals' representations of the task statements and the processes by which judgements are based on this representation, can explain why the 'causal' scenario entailed an increased reliance on the base-rate information in observed judgements. According to SSR theory, the changes in the 'causal' statement [28'b] would only affect the representation of sample space $\mathscr{S}_{1}$. Accordingly, the elements of $\mathscr{S}_{1}$ will no longer be characterised by intension "in_city" but will instead be characterised by intension "in_accident". It follows that samples $S_{1}$ and $S_{2}$ will be described by $\{c a b \wedge$ in_accident $\wedge$ green $\}$ and $\{c a b \wedge$ in_accident $\wedge$ blue $\}$, respectively. The representation of the task statements can thus be characterised as follows, 


$$
\begin{aligned}
& \mathscr{S}_{1}=\{\text { city_cab } \wedge \text { in_accident }\} \\
& \mathrm{S}_{1}=\{\text { city_cab } \wedge \text { in_accident } \wedge \text { green }\}- \\
& p\left(S_{1} \mid \mathscr{S}_{1}\right)=85 \% \\
& \mathrm{~S}_{2}=\{\text { city_cab } \wedge \text { in_accident } \wedge \text { blue }\} \\
& p\left(S_{2} \mid S_{1}\right)=15 \% \\
& S_{3}=\{\text { presented_cab } \wedge \text { blue }\} \quad \mathscr{S}_{2}=\{\text { presented_cab }\} \\
& p\left(S_{3} \mid \mathscr{S}_{2}\right)=50 \% \\
& \mathrm{~S}_{4}=\{\text { presented_cab } \wedge \text { green }\} \\
& p\left(\mathrm{~S}_{4} \mid \mathscr{S}_{2}\right)=50 \% \\
& \mathrm{~S}_{7}=\{\text { presented_cab } \wedge \text { green } \wedge \text { correctly_identified }\} \\
& p\left(S_{7} \mid S_{4}\right)=80 \% \\
& S_{8}=\{\text { presented_cab } \wedge \text { green } \wedge \text { incorrectly_identified }\} \\
& p\left(S_{8} \mid S_{4}\right)=20 \% \\
& \mathrm{~S}_{5}=\{\text { presented_cab } \wedge \text { blue } \wedge \text { correctly_identified }\} \\
& p\left(S_{5} \overline{\mid} S_{3}\right)=80 \% \\
& S_{6}=\{\text { presented_cab } \wedge \text { blue } \wedge \text { incorrectly_identified }\} \\
& p\left(S_{6} \mid S_{3}\right)=20 \% \\
& \_c a b \wedge \text { in_accident } \wedge \text { identified_blue } \wedge[\text { blue, } \sqcup \%]
\end{aligned}
$$

According to Proposition 3c of SSR theory, individuals will review their representation of the task's statements and base their judgement on the probability associated with the sample or model having the highest number of intensions matching those of the goal statement. The change in the formulation in the base-rate statement now results in sample $S_{2}$ having two intensions matching those of the goal statement, namely "in_accident" and "blue." Thus, on the basis of the propositions of SSR theory, one can predict that this task will increase the proportion of individuals relying on the base-rate information only, which is consistent with the results observed by Tversky and Kahneman (1980).

In the original cab problem, the inference that the conjunction of intensions "identified_blue" and "blue" was equivalent to the conjunction of "correctly_identified" and "blue" led individuals to answer $80 \%$, which corresponds to an inverse fallacy judgement. With the 'causal' scenario, the same inference results in both samples $S_{2}$ and $S_{5}$ having two intensions matching those of the goal statement. Indeed, the intensions of $S_{2}$ still match intensions "in_accident" and "blue" of the goal statements, whereas $S_{5}$ now matches intensions "correctly_identified" and "blue." Consequently, individuals who base their judgement on the updated goal statement will aggregate the probabilities 
associated with both samples $S_{2}$ and $S_{3}$; that is, they will aggregate $p(H)$ and $p(D \mid H)$. As noted earlier, a specific modal outcome is not to be expected in such situations because the ways in which the probability values will be aggregated (e.g., anchor and adjustment, multiplication, addition, etc.) will depend largely on individual abilities and preferences (Proposition 3c) rather than on general cognitive processes.

Thus, on the basis of the propositions of SSR theory, one can account for how participants made their judgements in both the standard cab problem and the causal cab problem, as well as explaining why the seemingly inconsequential change in the 'causal' scenario led to an increase of the proportion of individuals who provided judgements based on the base-rate value alone or whose value was intermediate between base-rate and inverse likelihood values, as reported by Tversky and Kahneman (1980). Additionally, whereas the authors' causality account did not specify the inducers of causal interpretations or the specific modalities by which causality determines the process by which judgements are made, the SSR account provides a formal framework of explanation and, hence, answer the criticisms made to the causality account.

\section{On the relevance account (Bar-Hillel, 1980)}

Bar-Hillel (1980) critiqued the causality explanation on the grounds that it lacked generalisability. She proposed that the effect of the causal scenario could be replicated even when causality could not be invoked. To test this hypothesis, she presented participants with the following scenario, now known as the 'intercom problem' (see BarHillel, 1980, p. 228),

[29a] $85 \%$ of the cabs in the city are Blue, and $15 \%$ are Green.

[29b] A cab was involved in a hit-and-run accident at night.

[29c] The wounded pedestrian (...) remembers hearing the sound of an intercom from the cab window.

[29d] Intercoms are installed in $80 \%$ of the Green cabs and in $20 \%$ of the Blue cabs.

[29e] What do you think are the chances that the errant cab was Green?

Bar-Hillel (1980) observed that this scenario was as efficient as Tverky and Kahneman's (1980) causal scenario in reducing the proportion of individuals committing the inverse fallacy. Bar-Hillel (1980) contended that these results were due to the fact that her formulation of the likelihood information (i.e., statement [29d]) had a low perceived relevance. Once more, however, this account was criticised herein (see chap. III, p. 59) because of its vagueness and lack of formalism.

The SSR account does not suffer from this shortcoming, yet its propositions can explain how the intercom problem led individuals to aggregate different probability 
values. The differences between the intercom problem and the original cab problem concern the characterisation of the datum and that of the likelihood information. Namely, in the intercom problem, the datum reads "The wounded pedestrian (...) remembers hearing the sound of an intercom from the cab window" instead of "a witness identified the cab as a Blue cab" and the likelihood information reads "Intercoms are installed in $80 \%$ of the Green cabs and in $20 \%$ of the Blue cabs" instead of "When presented with a sample of cabs (half of which were Blue and half of which were Green) the witness made correct identifications in $80 \%$ of the cases and erred in $20 \%$ of the cases." Statement [29a] defines the reference set $\mathscr{S}_{1}=\{$ city_cabs $\}$ and two subsets, $S_{1}=$ $\left\{c i t y \_c a b \wedge b l u e\right\}$ and $S_{2}=\left\{c i t y \_c a b \wedge\right.$ green $\}$. Statement [29d] defines two reference sets, $\mathscr{S}_{2}=\{c a b \wedge$ green $\}$ and $\mathscr{S}_{3}=\{c a b \wedge b l u e\}$ and two respective subsets $S_{3}=\{c a b \wedge$ green $\wedge$ intercom $\}$ and $S_{4}=\{c a b \wedge$ blue $\wedge$ intercom $\}$. Assuming that individuals infer that 'the Green cabs' and 'the Blue cabs' defined in statement [29d] are cabs from the city (i.e., can be characterised by the intension "city_cab"), $\mathscr{T}_{2}$ and $S_{2}$ can be assimilated and $\mathrm{S}_{3}$ can be represented as a sample included in $S_{2}$. Similarly, $\mathscr{S}_{3}$ and $S_{1}$ can be assimilated and $S_{4}$ can be represented as a sample included in $S_{1}$. The resulting representation of the intercom task is graphically characterised below,

$$
\begin{gathered}
S_{3}=\left\{c i t y \_c a b \wedge \text { green } \wedge \text { intercom }\right\} \\
p\left(S_{3} \mid S_{2}\right)=80 \% \\
S_{4}=\{\text { city_cab } \wedge \text { blue } \wedge \text { intercom }\} \\
p\left(S_{4} \mid S_{1}\right)=20 \% \\
S_{1}=\left\{c i t y \_c a b\right\} \\
p\left(S_{1} \mid \mathscr{S}_{1}\right)=85 \% \\
S_{2}=\left\{c i t y \_c a b \wedge \text { green }\right\} \\
p\left(S_{2} \mid \mathscr{S}_{1}\right)=15 \%
\end{gathered}
$$

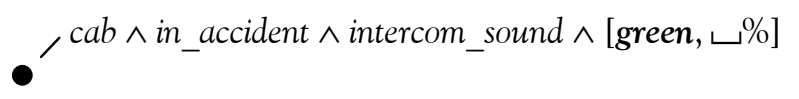

$(29 b, c, e)$

According to Proposition 3c, individuals will base their judgement on the probability associated with the sample or samples whose intensions match those of the goal statement to the greater extent. In this case, there are two such samples, namely samples $\mathrm{S}_{2}$ and $\mathrm{S}_{3}$ which both match the intension "green." SSR theory therefore predicts that most judgements in this task will be based on an aggregation of $p\left(S_{3} \mid S_{2}\right)$ and $p\left(S_{2} \mid \mathscr{S}_{1}\right)$. Even if individuals do not infer that the green and blue cabs defined in statement [29d] are indeed city cabs and, hence, build a representation of the statements of the task based on multiple sample spaces (i.e, by representing $S_{3}=\{$ green_cab $\wedge$ intercom $\}$ in a 
new sample space $\mathscr{T}_{2}=\{$ green_cab $\}$ and by representing $S_{4}=\{$ blue_cab $\wedge$ intercom $\}$ in yet another sample space $\mathscr{S}_{3}=\{$ blue_cab $\}$, judgements would still be expected to aggregate the corresponding base-rate and inverse likelihood information, thus defined as $p\left(S_{2} \mid \mathscr{S}_{1}\right)$ and $p\left(S_{3} \mid \mathscr{S}_{2}\right)$, respectively. This is because of the presence of an elementary goal statement (Proposition 3b). It is only when the goal statement is a set statement and, hence, when individuals will relate the different samples identified to a common sample space (Proposition 3c), that the type of representation (multiple sample vs. fully inclusive) will affect the judgement observed.

The prediction of the aggregation of $p\left(S_{3} \mid S_{2}\right)$ and $p\left(S_{2} \mid \mathscr{S}_{1}\right)$ is consistent with the results observed by Bar-Hillel (1980) who noted that the distribution of judgements obtained following the administration of the intercom cab problem was "flatter, 'noisier', suggesting that there is no prevailing strategy of integration favoured by a large proportion of [participants]" (p. 228). The author argued that those results were due to the fact that the 'indicant' information (i.e., the proportion of Blue and Green cabs equipped with an intercom) was perceived to be as irrelevant as base-rate information (i.e., the proportion of Blue and Green cabs in the city). The author openly justified this effect by the manipulation of what she believed would affect individuals' judgements of relevance (Bar-Hillel, 1980, p. 230). SSR theory therefore formalises Bar-Hillel's intuition about 'relevance'.

Interestingly, this SSR interpretation also suggests that if participants had been told that the taxi involved in the accident indeed had an intercom installed; that is, if the goal statement was represented as,

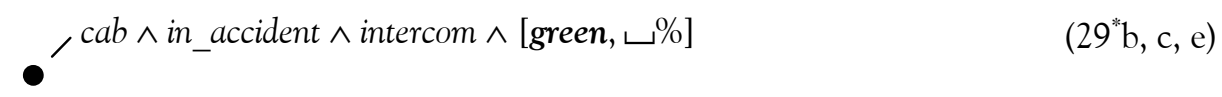

SSR theory would predict that most participants will base their judgement solely on the probability associated with sample $S_{3}$ because it would then be the only sample whose description matches two intensions of the goal statement (namely, "intercom" and "green"). As a result, judgements would then be most likely to be based on $p\left(S_{3} \mid S_{2}\right)$ alone, thereby increasing the proportion of inverse fallacy judgements observed.

\section{On the partitive account (Macchi, 1995, 2000)}

The latest interpretational account that has been proposed to explain the origin of posterior probability judgements in the Textbook-problem paradigm is Macchi's (1995) 'partitive' account. As reviewed in Chapter III (see p. 60), Macchi (1995) suggested that 
neither considerations of causality nor considerations of relevance explained the effects observed by Tversky and Kahneman (1980) and Bar-Hillel, (1980). Macchi argued instead that those effects were due to the 'partitive' nature of the causal and intercom scenarios. In a partitive text, the author argued, the specific information (i.e., the likelihood of observing $D$ when $H$ is true) defines a subset that is considered as part of a global set, which is itself defined by the base-rate information. Yet, Macchi only defined the attributes of partitiveness (i.e., what a partitive structure entails) and relied on exemples to characterise the essence of this concept (i.e., what a partitive text consists of) rather than on a formal definition. Hence, the partitive account cannot be taken as a satisfactory algorithmic account of posterior probability judgements; that is, it does not explain how individuals' representations of the task and their cognitive processes interact to produce their judgements. By contrast, once more, the propositions of SSR theory do provide such an explanation while still accounting for results observed with 'partitive' tasks. For instance, consider Macchi's (1995, Appendix, p. 199) 'partititive and complementary' version of the cab problem, which involved the following statements:

[30a] In the city $85 \%$ of the cabs are Green and $15 \%$ are Blue.

[30b] A witness recognised as Blue $80 \%$ of the Blue cabs and mistook 20\% of the Green cabs for Blue ones.

[30c] Among those cabs indicated as Blue, what is the percentage of cabs that really are Blue?

The author found that only $10 \%$ of participants solely relied on the inverse likelihood information [i.e., $p(D \mid H)=80 \%$ ] and that "nearly all of them [the participants] computed the posterior probability correctly" (Macchi, 1995, p. 199).

Statement [30a] defines the reference set $\mathscr{S}_{1}=\{$ city_cabs $\}$ and two subsets, $S_{1}=$ $\left\{c i t y \_c a b \wedge\right.$ green $\}$ and $S_{2}=\left\{c i t y \_c a b \wedge\right.$ blue $\}$. Statement [30b] defines two reference sets, $\mathscr{S}_{2}=\{c a b \wedge b l u e\}$ and $\mathscr{S}_{3}=\{c a b \wedge$ green $\}$ and two respective subsets $S_{3}=\{c a b \wedge$ blue $\wedge$ recognised_blue $\}$ and $S_{4}=\{c a b \wedge$ green $\wedge$ mistaken_blue $\}$. Assuming that individuals infer that "the Green cabs" and "the Blue cabs" defined in statement [30b] and "those cabs indicated as Blue" in statement [30c] are all cabs from the city (i.e., can be characterised by the intension "city_cab"), $\mathscr{T}_{2}$ and $S_{2}$ can be assimilated and $S_{3}$ can be represented as a sample included in $S_{2}$. Similarly, $\mathscr{S}_{3}$ and $S_{1}$ can be assimilated and $S_{4}$ can be represented as a sample included in $S_{1}$. The resulting representation of the partitive task is graphically characterised below, 


$$
\begin{gathered}
S_{3}=\{\text { city_cab } \wedge \text { blue } \wedge \text { recognised_blue }\} \\
p\left(S_{3} \mid S_{2}\right)=80 \% \\
S_{4}=\{\text { city_cab } \wedge \text { green } \wedge \text { mistaken_blue }\} \\
p\left(S_{4} \mid S_{1}\right)=20 \% \\
S_{1}=\left\{c i t y \_c a b \wedge \text { green }\right\} \\
p\left(S_{1} \mid \mathscr{S}_{1}\right)=85 \% \\
S_{2}=\left\{c i t y \_c a b \wedge b l u e\right\} \\
p\left(S_{2} \mid \mathscr{S}_{1}\right)=15 \%
\end{gathered}
$$

Unlike the Tversky and Kahneman (1980) and Bar-Hillel (1980) tasks, the goal statement of Macchi's (1995) 'partitive' task was a set statement. Statement [30c] indeed calls for the evaluation of the proportion of elements in a subset $S_{1}=\{$ city_cab $\wedge$ indicated_blue $\wedge$ blue $\}$ relative to the reference set $\mathscr{S}=\left\{c i t y \_c a b \wedge\right.$ indicated_blue $\}$.

According to Proposition 3a of SSR theory, individuals will form their judgement on the basis of the samples or elements whose intensions match those of the goal statement. Thus, individuals would be expected to evaluate the proportion of elements in the samples whose intensions match those of subset $S$ relative to the proportion of elements in the samples whose intensions match those of reference set $\mathscr{S}$. A priori, there is no sample in the representation of the statements of this problem that can be identified with either $S$ or $\mathscr{S}$ Y Yet, there is a readily available inference that will improve matching. Namely, it can be inferred that both intension "recognised_blue" and intension "mistaken_blue" are equivalent to the intension 'indicated_blue.' Proposition 3b accounts for such an inference (Proposition 3b). This interpretation would in turn lead individuals to infer that $\mathscr{S}=S_{3} \cup S_{4}$ and that $S=S_{3}$. Consequently, their representation of the task would thus become, 


$$
\begin{aligned}
& \mathscr{S}_{1}=\left\{c i t y \_c a b\right\} \\
& \mathrm{S}_{3}=\{\text { city_cab } \wedge \text { blue } \wedge \text { indicated_blue }\} \\
& p\left(S_{3} \mid S_{2}\right)=80 \% \\
& S_{4}=\left\{c i t y \_c a b \wedge \text { green } \wedge \text { indicated_blue }\right\} \\
& p\left(S_{4} \mid S_{1}\right)=20 \% \\
& \mathrm{~S}_{1}=\left\{c i t y \_c a b \wedge \text { green }\right\}^{\prime} \\
& p\left(S_{1} \mid \mathscr{S}_{1}\right)=85 \% \\
& \mathrm{~S}_{2}=\{\text { city cab } \wedge \text { blue }\} \\
& \{\text { city_cab } \wedge \text { indicated_blue }\}=\mathscr{S} \\
& \therefore \mathscr{S}=S_{3} \cup S_{4} \\
& \{\text { city_cab } \wedge \text { indicated_blue } \wedge \text { blue }\}=S \\
& \therefore S=S_{3} \\
& \therefore p(S \mid \mathscr{S})=p\left(S_{3} \mid S_{3} \cup S_{4}\right)=\sqcup \%
\end{aligned}
$$$$
p\left(S_{2} \mid \mathscr{T}_{1}\right)=15 \%
$$

Proposition $3 \mathrm{e}$ suggests that since the proportions associated with $S_{3}$ and $S_{4}$ have different referents, individuals will first relate these two samples to a common sample space, namely, $\mathscr{S}_{1}$ (i.e., by evaluating the proportion of elements in each of these samples relative to the size of $\mathscr{S}_{1}$ ) before they evaluate the proportion of elements in $S_{3}$ relative to the union of $S_{3}$ and $S_{4}$. Provided that they correctly infer those proportions, individuals relying on this representation should provide Bayesian judgements (Corollary 1).

Thus, it was possible to explain why, and hence predict that, Macchi's (1995) partitive task entailed high proportions of Bayesian judgements, on the basis of a fully formalised account of the representation underlying the task at hand, and the cognitive processes underlying the judgements observed. This analysis also shows that the 'partitive' text proposed by Macchi (1995) may only lead individuals to make correct judgements if they appropriately infer the relations between different intensions (namely, if they infer that all cabs mentioned belong to the sample space of cabs in the city and if they infer that both city cabs defined as "recognised as Blue" and "mistaken for Blue") are both cabs that can be described as "indicated as Blue". It follows that a text that makes these inferences redundant as in,

[31a] $85 \%$ of the cabs in the city are Green and $15 \%$ are Blue.

[31b] A witness indicated as Blue $80 \%$ of the Blue cabs in the city. However, he also indicated as Blue $20 \%$ of the Green cabs in the city.

[31c] Among those cabs indicated as Blue by the witness, what is the percentage of cabs that really are Blue?

where the explicit use of consistent intensions to uniquely define sets may facilitate the successful completion of the task. Alternatively, a text that increases ambiguity, as in, 
[32a] In the city $85 \%$ of the cabs are Green and $15 \%$ are Blue.

[32b] A witness recognised as Blue $80 \%$ of Blue cabs and mistook $20 \%$ of Green cabs for Blue ones.

[32c] Among cabs indicated as Blue, what is the percentage of cabs that really are Blue?

where the suppression of the determinative cues 'the' and 'those' used to indicate that the cabs defined in both likelihood and goal statements (i.e., statements [32b] and [32c], respectively) belong to the sample space defined by the base-rate statement (i.e., statement [32a]), may entail a significant decrease in the proportion of Bayesian judgements observed. Whereas Macchi's (1995) partitive account does not explicitly predict that such variations would affect performance, they are both accounted for and predicted by the SSR theory, whose specific predictions are thus open to refutation in future empirical tests.

\section{On the Mental Model theory of naïve probability judgements (Johnson-Laird et al., 1999)}

\section{Can samples be 'natural' mental models?}

SSR theory is based on the assumption that individuals represent samples as well as individual discrete elements. As such, it challenges one of the basic assumptions of Mental Model theory (Johnson-Laird, 1983). Johnson-Laird (1983, see also JohnsonLaird \& Byrne, 1993) indeed argued that it was psychologically implausible to claim that individuals would represent information based on sample representations. According to MMT, mental models are based on a representation of finite sets of discrete entities or 'mental tokens'. Johnson-Laird (1983) argued that "neither Euler circles nor Venn diagrams are natural mental models" (p. 93) and instead characterised them as complex mathematical notations. For instance, to represent a statement such as 'Some artists are beekeepers,' Johnson-Laird explained that one would need to represent four separate diagrams of Euler circles since this assertion does not rule out the possibility that all artists are beekeepers (See Figure VII-5, adapted from Johnson-Laird, 1983, p. 78).

\section{'(At least) Some artists are beekeepers'}

Fully explicit representation based on Euler circles

(i)

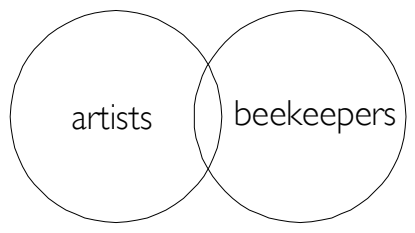

(ii)

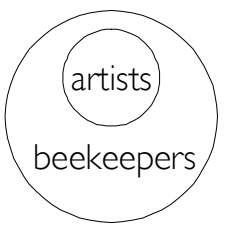

(iii)

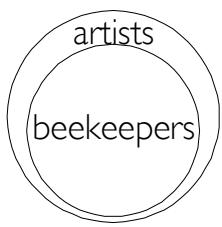

(iv)

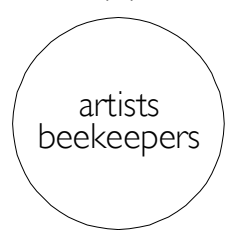

Figure VII-5 - Fully explicit representations based on Euler circles of the statement "some artists are beekeepers." 
Johnson-Laird's criticism was based on an appraisal of existing theories of syllogistic reasoning (e.g., Erickson, 1974 as cited by Johnson-Laird, 1983; Newell, 1981, ibid.). These theories postulated that human inferences rely on fully explicit sample representations. Johnson-Laird (1983) demonstrated that such assumptions are implausible from a psychological standpoint because they assume individuals to represent all the contingencies implied by a statement as suggested in Figure VII-5.

In contrast, MMT proposes that a 'natural' representation is a model where only the true possibilities are explicitly represented. Other possibilities remained implicit and are formalised by the use of three dots. For example, a 'natural' mental model for the statement 'Some artists are beekeepers' is of the form:

$$
\begin{array}{ll}
\text { artist } & \text { beekeeper } \\
\text { artist } & \text { beekeeper }
\end{array}
$$

If the implicit models were made explicit, the representation could then become:

$$
\begin{array}{rr}
\text { artist } & \text { beekeeper } \\
\text { artist } & \text { beekeeper } \\
\text { artist } & \neg \text { beekeeper } \\
\neg \text { artist } & \text { beekeeper } \\
\neg \text { artist } & \neg \text { beekeeper }
\end{array}
$$

But, according to MMT, this latter representation is unlikely to be elicited, because of its associated workload on working memory. SSR theory shares this assumption with MMT as it is based on the assumption that individuals will initially represent what is explicitly described in a statement. However, SSR theory allows for representations to be updated following either the processing of a subsequent task statement or an individual's inference regarding a given statement. Additionally, SSR theory distinguishes two different types of representation (element-based vs. sample-based). Namely, according to SSR theory, the statement 'some artists are beekeepers' will involve the representation of a sample space $\mathscr{S}_{1}$ encompassing all elements having the intension $i(x)=$ " $x$ is an artist." Included within $\mathscr{S}_{1}$, there will be sample $S_{1}=\{$ artist $\wedge$ beekeper $\}$. Such representation can thus be formalised as follows ${ }^{2}$ :

${ }^{2}$ Note that the Sample Space Representation account would imply that a different representation would be elicited by the statement 'Some beekeepers are artists' where the sample space $\mathscr{S}$ would be defined by $\mathscr{S}=\{$ beekeepers $\}$. 


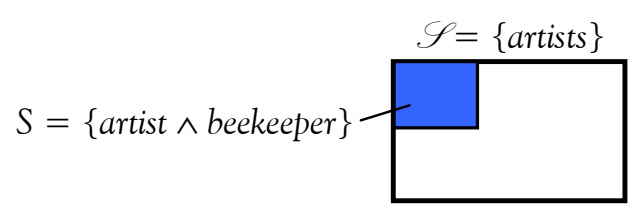

Thus, SSR theory answers Johnson-Laird's criticisms made to the representations suggested by earlier syllogistic theories (Johnson-Laird, 1983, p. 77 onwards). Indeed, SSR theory does not assume that individuals form fully explicit representations. However, unlike MMT, SSR theory does not rule out the possibility that individuals may rely on sample-based representations as well as element-based representations. The next section will show that, by allowing both sample and discrete elements to be represented, SSR theory overcome the shortcomings associated with an account of posterior probabilities judgements based of MMT.

\section{SSR theory vs. MMT applied to probabilistic reasoning}

In Set theory, sets can be described either by extension, i.e., by listing all their individual members, or by intension, i.e., by referring to the specific description used to define membership. MMT of probabilistic reasoning is an exclusively extensional account because it relies on the assumption that individuals will always represent the discrete entities of a set rather than the set itself. In contrast, SSR theory assumes that representations may rely both on extensional and intensional descriptions.

As noted earlier (see chap. III, p. 74 onwards), MMT provides a compelling explanation for the processes underlying reasoning about possibilities (e.g., to represent the statement 'there is a circle or there is a square' or 'there is a chance in six that a dice will show three dots'). Yet it was suggested that MMT in its current state cannot always satisfactorily account for the empirical results observed within studies of posterior probability judgements. In particular, one shortcoming identified was the fact that the MMT of naïve probabilistic reasoning stipulated that numerical information had to be in an absolute format for the elicitation of Bayesian judgements to be possible. MMT indeed states that if (and only if) models are tagged with absolute frequencies or absolute chance values, people will use the subset principle to estimate the posterior probability. Yet, this requisite is at odds with empirical results reported within the literature (e.g., see Macchi, 2000). In contrast, the propositions of SSR theory explain why even relative numerical formats may allow for Bayesian judgements to be elicited, as demonstrated in the previous section. 
Additionally, it was noted that the MMT did not provide a theoretical justification for this limitation of the application of the subset principle to absolute quantities. Indeed, the core principles of Mental Model theory cannot account for this restriction. In contrast, the propositions of SSR theory provide a clear-cut explanation for the origin (and hence the prediction) of Bayesian judgements. Additionally, these propositions can be applied to tasks that entail the elicitation of non-Bayesian judgements and still explain and predict the judgements that are likely to be observed. For instance, it was demonstrated earlier that the propositions of SSR theory explain why, in tasks solely based on elementary probability statements, individuals are most likely either to commit the inverse fallacy or to rely on both $p(D \mid H)$ and $p(D \mid \sim H)$, but will tend not to integrate the base-rate information. The propositions of SSR theory also explain why similar judgements will be observed in tasks where the premises are based on set statements, but where the goal statement is an elementary statement.

The MMT account of posterior probability judgements also stipulates that posterior probability judgements will be in line with Bayesian prescriptions if the application of the subset principle allows for the evaluation of the correct posterior probability (JohnsonLaird et al., 1999, p. 81). This argument allows the theory to account for the fact that multiple Set-based tasks presented in an absolute frequency format may entail the application of the subset principle but would not generally lead to the elicitation of Bayesian judgements.

Girotto and Gonzalez (2001) suggested that the difficulty in multiple Set-based tasks lies in its reference to three separate groups of observations whereas partitioned Setbased tasks refer to a set partitioned into exhaustive subsets. Such argument, however, cannot be accounted for by MMT: we saw earlier that one of the basic assumptions of this theory is indeed that representations rely on separate elements rather than on samples of elements. Accordingly, whether a task refers to a unique sample or several separate samples should not make a difference. In contrast, SSR theory not only validates Girotto and Gonzalez (2001)'s argument, but also explains why this happens to be the case. For example, consider the following set of statements, taken from the disease problem used by Girotto and Gonzalez (2001, p. 253): 
[33a] A person who was tested had 4 chances out of 100 of having the infection.

[33b] 3 of the 4 chances of having the infection were associated with a positive reaction to the test.

[33c] 12 of the remaining 96 chances of not having the infection were also associated with a positive reaction to the test.

[33d] Imagine that Pierre is tested now. Out of a total of 100 chances, Pierre has chances of having a positive reaction, of which will be associated with having the infection.

The authors found that $43 \%(N=40)$ participants provided the correct answer to this task. This problem will entail a representation based on samples as all its statements are set statements as opposed to elementary statements (Proposition 1b). The representation of the task will be fully inclusive (Corollary 1 ). The representation of the problem can be formally characterised as follows,

$$
\begin{gathered}
\mathscr{S}_{1}=\{\text { chance }\} \\
S_{3}=\{\text { chance } \wedge \text { infection } \wedge \text { positive_reaction }\} \\
n\left(S_{3}\right)=3 \\
S_{4}=\{\text { chance } \wedge \text { infection } \wedge \text { positive_reaction }\} \\
n\left(S_{4}\right)=12 \\
S_{1}=\left\{\begin{array}{c}
n \text { chance } \wedge \text { infection }\} \\
n\left(S_{1}\right)=4 \\
\left.S_{2}=\text { chance } \wedge \neg \text { infection }\right\} \\
n\left(S_{2}\right)=96
\end{array}\right.
\end{gathered}
$$

$$
\begin{gathered}
\{\text { chances }\}=\mathscr{S} \\
\therefore \mathscr{S}=\mathscr{S}_{1}
\end{gathered}
$$$$
\{\text { chance } \wedge \text { positive_reaction }\}=S
$$

$$
\therefore S=S_{3} \cup S_{4}
$$

$\{$ chance $\wedge$ positive_reaction $\wedge$ infection $\}=S_{*}$

$n(S)$ out of which $n\left(S_{*}\right)=\sqcup$ out of which $\sqcup$

$\therefore n\left(S_{3} \cup S_{4}\right)$ out of which $n\left(S_{3}\right)=3+12$ out of which 3

The goal statement defines the set $S=\{$ chance $\wedge$ positive_reaction $\}$ and its subset $S_{*}=\{$ chance $\wedge$ positive_reaction $\wedge$ infection $\}$. SSR theory specifies that individuals will review their representation of the task and base their judgements on the samples whose intensions match those of the goal statement (Proposition 3a). In the present case, such samples are defined by $S_{3}$ and $S_{4}$, respectively. The goal statement will thus lead individuals to focus on these two samples within their representation of the task premises, and to base their judgements on the evaluation of the number of elements in both $S_{3}$ and $S_{4}\left[n\left(S_{3} \cup S_{4}\right)\right]$ and the evaluation of the number of elements in $S_{3}\left[n\left(S_{3}\right)\right]$. This explains why most individuals are able to answer fully inclusive Set-based problems 
correctly. Note that this is consistent with Corollary 1 of the theory as fully inclusive Set-based tasks satisfy conditions (i), (ii), (iii) and (iv-c) of this corollary (see p. 215).

Girotto and Gonzalez (2001) also predicted that a problem consisting of statements [33a], [33b] and [33c] (see p. 233) and a "single-step" goal statement of the form,

[33d'] If Pierre has a positive reaction, there will be chance(s) out of that the infection is associated with his positive reaction.

would not lead to a correct estimate. The authors argued that such a question would not prompt judges to apply the subset principle as defined by MMT (Johnson-Laird et al., 1999). Their predictions were confirmed in the sense that this "single-step" question elicited very low rates of Bayesian answers $(8 \%, N=40)$. The authors observed that this goal statement led most individuals to evaluate the number of elements both described by intensions "positive_reaction" and "infection" out of a total of 100 . This illustrates how SSR theory extends on the predictive power of MMT: whereas on the basis of the principles of MMT, one can only predict that individuals will not provide Bayesian answers with "single-step" goal statements; on the basis of the propositions of SSR theory, one can not only predict that such a "single-step" goal statement will not elicit high proportions of Bayesian answers but also predict what answers are most likely to be observed.

Goal statement [33d'] indeed focuses on the set of chances of an infection associated with a positive reaction. Namely, the goal statement defines the subset $S=\{$ chance $\wedge$ infection $\wedge$ positive_reaction $\}$ and the reference set $\mathscr{S}=\{$ chance $\}$. Accordingly, this task would elicit the following representation, 


$$
\begin{gathered}
\mathscr{S}_{1}=\{\text { chance }\} \\
S_{3}=\{\text { chance } \wedge \text { infection } \wedge \text { positive_reaction }\} \\
n\left(S_{3}\right)=3 \\
S_{1}=\{\text { chance } \wedge \text { infection } \wedge \text { positive_reaction }\} \\
\left.n\left(S_{4}\right)=12 \wedge \text { infection }\right\} \\
n\left(S_{1}\right)=4 \\
\left.S_{2}=\text { chance } \wedge \neg \text { infection }\right\} \\
n\left(S_{2}\right)=96 \\
\{\text { chances }\}=\mathscr{S} \\
\therefore \mathscr{S}=\mathscr{S} \mathscr{S}_{1} \\
\{\text { chance } \wedge \text { positive_reaction } \wedge \text { infection }\}=S \\
\therefore S=S_{3} \\
n(S) \text { out of } n(\mathscr{S})=\sqcup \text { out of } \sqcup \\
\therefore n\left(S_{3}\right) \text { out of } n\left(\mathscr{S}_{1}\right)=3 \text { out of } 100
\end{gathered}
$$

There is only one sample matching the intension of the subset of the goal statement, namely sample $S_{3}$, which contains the elements described by "chance" and "positive_reaction" that are also described by "infected." Furthermore, the intension of the sample space in the representation of the task statements $\left(\mathscr{S}_{1}\right)$ matches that of the reference set defined in the goal statement (namely "chance"). SSR theory predicts that individuals will therefore evaluate the number of elements in $S_{3}$ relative to the number of elements in $\mathscr{S}_{1}$ (Proposition $3 \mathrm{~d}$ ) resulting in the judgement ' 3 out of 100 ' in this case; a prediction that is therefore confirmed by the empirical results observed by Girotto and Gonzalez (2001, p. 256).

Now, consider the corresponding multiple sample frequency task, also taken from Girotto and Gonzalez (2001, p. 263).

[34a] 4 out of 100 people were infected.

[34b] 75 out of 100 infected people had a positive reaction to the test.

[34c] 25 out of 100 uninfected people also had a positive reaction to the test.

[34d] Among people having a positive reaction to the test, out of will have the infection.

As for the previous problem, this problem will entail a representation based on samples as all its statements are set statements (Proposition 1c). This problem involves three intensions, namely 'person,' 'infected,' and 'positive_reaction.' The full representation of the task can thus be formalised as follows, 


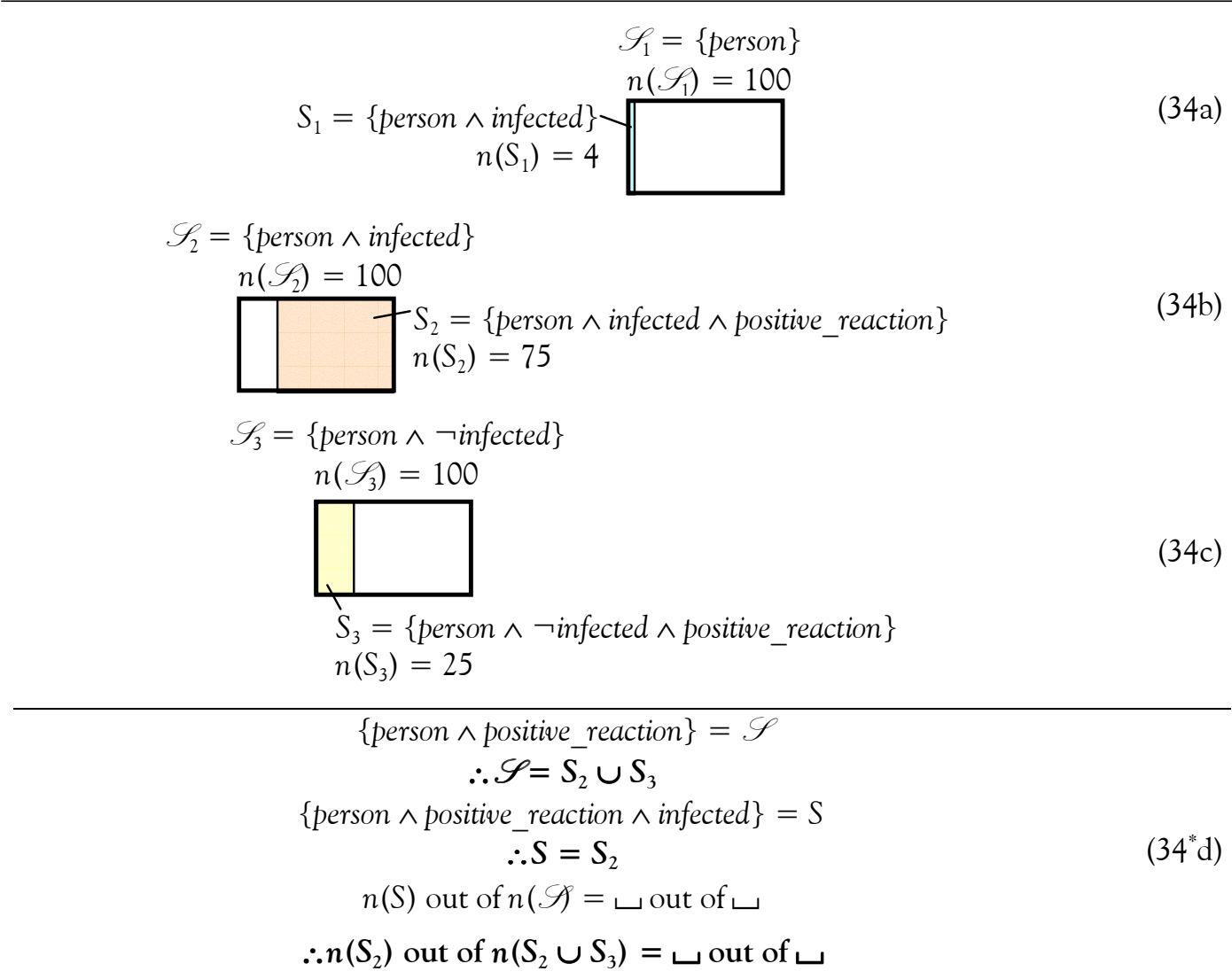

The violation of the numerical condition for inclusion will prevent the assimilation of $\mathscr{S}_{2}$ with $S_{1}$ and that of $\mathscr{S}_{3}$ with $\sim S_{1}$ (the complementary set of $S_{1}$ ) (Proposition $2 c$, condition ii).

Proposition 3a of SSR theory implies that individuals will review their representation of the statements of the task and form their judgement based on the sample or samples whose intensions match those of the goal statement. In the present case, the goal statement points individuals to the sample $S_{2}$ and the union of samples $S_{2}$ and $S_{3}$ (Proposition 3a). It follows that individuals will update their representation to combine or assimilate $\mathscr{S}_{2}$ and $\mathscr{S}_{3}$ (Proposition 3 e), and evaluate the number of elements characterised by intensions "person", "positive_reaction", and "infected" [i.e., $n\left(S_{2}\right)=75$ ] relative to the number of elements characterised by intensions "person" and "positive_reaction" [i.e., $\left.n\left(S_{2}\right)+n\left(S_{3}\right)=75+25=100\right]$ (Proposition 3d).

Whether individuals combine or assimilate the two sample spaces would not affect the final judgement since quantity information is expressed in an absolute format. Combination would lead to $n(\mathscr{Y})=200$ whereas assimilation would lead to $n(\mathscr{Y})=100$. Assimilation may be more 'natural' here since the intension "uninfected" is likely to be interpreted as " $\neg$ infected", thereby leading individuals to believe that $S_{3}$ is in fact the complement of $S_{2}$. Alternatively, combination would have been more likely if $n\left(S_{3}\right)$ was 
more than 25 because the sum of $n\left(S_{3}\right)+n\left(S_{2}\right)$ would then have been greater than the size of the sample space $\mathscr{T}_{2}$.

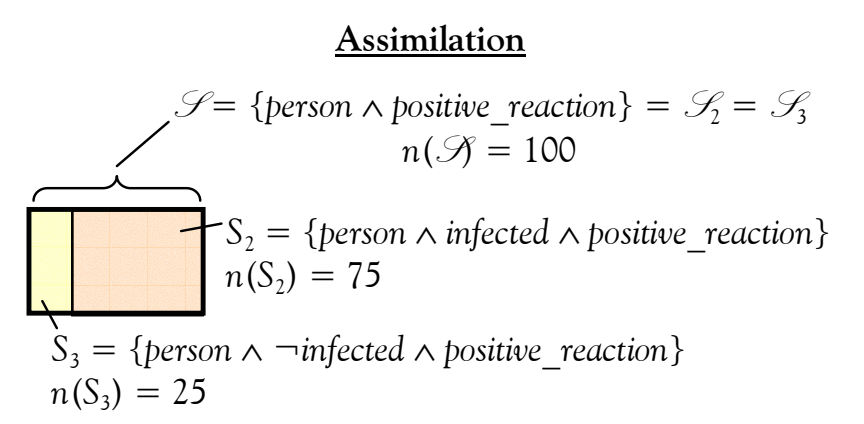

\section{Combination}

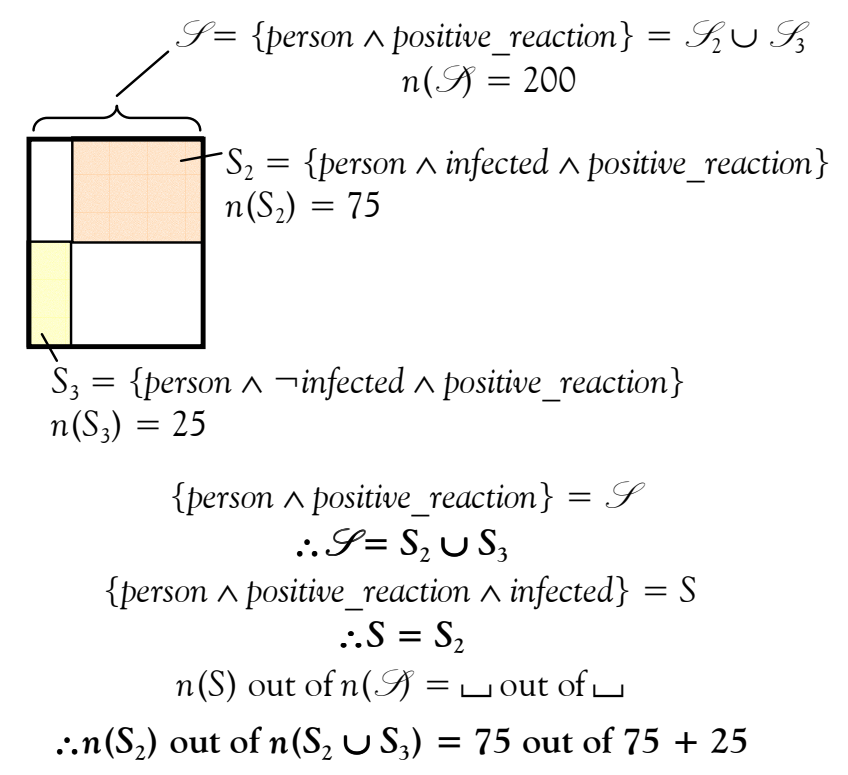

$\left(34^{t} \mathrm{~b}, \mathrm{c}\right)$

This prediction is, again, confirmed by the results observed by Girotto and Gonzalez (2001) with this particular task. Although the outcome of judgement is identical to an inverse fallacy judgement based on sample space $\mathscr{S}_{2}$ alone, SSR theory shows that the judgemental processes underlying this particular task are quite different, taking into account elements from two different sample spaces (namely, $\mathscr{S}_{2}$ and $\mathscr{S}_{3}$ ). Consequently, when the total number of elements in $S_{2}$ and $S_{3}$ is not 100 SSR theory would predict that most judgements observed will have a value that differs from the value of an inverse fallacy judgement. For example, if $n\left(S_{3}\right)=40$, SSR theory would predict that most individuals would answer '75 out of 115' in the above-mentioned task.

In sum, like MMT of naïve probability judgements, the propositions of SSR theory provide a specific explanation with regards to the origin of Bayesian posterior probability judgements. The propositions of SSR also overcome the shortcomings raised by the application of MMT to explain posterior probability judgements. These propositions indeed account for the observation of Bayesian judgements in tasks framed in relative 
frequency formats, thus accounting for existing empirical results (e.g., Macchi, 2000) that are in conflict with the prediction of the 'subset principle' of MMT. SSR theory also extends on the predictive scope of MMT as the propositions of SSR theory can account for the processes underlying judgements observed in both the Textbook-problem paradigm and the Set-based paradigm, whether or not Bayesian.

The next section demonstrates how the empirical results observed in this thesis both within the Textbook-problem (chap. V) and the Set-based paradigm (chap. VI) can be accounted for by the propositions of SSR theory.

\section{ACCOUNTING FOR EMPIRICAL FINDINGS \\ OF THE THESIS WITH SSR THEORY}

\section{Inverse fallacy and complementary goal statements}

In Chapter V, the inverse fallacy was established as the main judgement method applied to solve Textbook problems such as the Glom-Fizo task. From a process-focused perspective, such identification should be followed by the development of a theoretical account explaining why these tasks lead to the application of such a method. In Chapter III, it was suggested that existing accounts (i.e., the 'causality account,' Tversky \& Kahneman, 1980; the 'specificity account,' Bar-Hillel, 1980; or the 'partitive account,' Macchi, 1995, 2000) were not formalised enough to adequately explain the exact modalities by which the inverse fallacy was triggered by the task. In the previous section of the present chapter, it was shown how SSR theory can answer the criticisms made of these earlier accounts, while predicting what judgement outputs are most likely to be observed.

Experiment 4 (see chap. V) demonstrated that when individuals are asked to judge $p(H \mid D)$ and $p(\sim H \mid D)$ together in sequence, they are more likely to commit the inverse fallacy than when they are only asked to estimate $p(H \mid D)$ or $p(\sim H \mid D)$ but not both. Whereas existing accounts of judgements within the Textbook-problem paradigm cannot account for this finding, by contrast, one can explain this finding on the basis of the propositions of the SSR theory. Indeed, when the goal statement of a posterior probability judgement task is elementary (i.e., refers to a single element), Proposition 3c of SSR theory suggests that individuals will either commit the inverse fallacy or aggregate the probabilities associated with $p(D \mid H)$ and $p(D \mid \sim H)$ if they judge that an 
element described as having $D$ may either belong to a category $H$ or its alternative $\sim H$ (see p. 207).

For example, consider the one-goal Glom-Fizo task used in Experiment 4 (see chap. $\mathrm{V})$. The statements of this task can be summarised as follows,

[35a] There are 1 million Gloms and 1 million Fizos on Vuma.

[35b] 98\% Gloms play the harmonica.

[35c] 58\% Fizos play the harmonica.

[35d] A creature plays the harmonica.

The goal statements are then defined by the question:

[35e] Estimate the chances in 100 that this creature is a Glom.

This task involves intensions $i_{1}(x)=$ ' $x$ is a Glom', $i_{2}(x)=$ ' $x$ is a Fizo', and $i_{3}(x)=$ ' $x$ plays the harmonica'. According to SSR theory (Proposition 2c), such a task would give rise to the following representation,

$$
\begin{gathered}
\mathscr{S}_{1}=\{\text { Glom }\} \\
n\left(\mathscr{S}_{1}\right)=1 \text { million } \\
S_{1}=\{\text { Glom } \wedge \text { plays_harmonica }\}
\end{gathered}
$$$$
p\left(S_{1} \mid \mathscr{T}_{1}\right)=98 \%
$$

$$
\begin{aligned}
\mathscr{S}_{2} & =\{\text { Fizo }\} \\
n\left(\mathscr{S}_{2}\right) & =1 \text { millio } \\
S_{2}=\{\text { Fizo } \wedge \text { plays_harmonica }\} &
\end{aligned}
$$$$
p\left(S_{2} \mid \mathscr{S}_{2}\right)=58 \%
$$

$$
\text { ¿ creature } \wedge \text { play_harmonica } \wedge \text { [Glom, } \longleftarrow \text { \%] }
$$

Following from Proposition 3c, individuals may provide an answer based on the probability associated with sample $S_{1}$ as this is the sample whose intensions match the highest number of intensions of the goal statement. This answer corresponds to an inverse fallacy. Alternatively, individuals may infer that the creature could also be a Fizo, and (ii) update their representation of the goal statement accordingly (Proposition $3 b)$. The representation ( $35 \mathrm{~d}$ ) of the goal statement will thus be updated to,

$$
\text { ¿ creature } \wedge \text { play_harmonica } \wedge \text { [Glom } \vee \text { Fizo, }\llcorner \%]
$$

This in turn, would lead individuals to base their final judgement on the aggregation of the probabilities associated with samples $S_{1}$ and $S_{2}$. This is because the intensions of both samples now match two of the intensions of the goal statement.

Now, consider the case where participants are asked to judge both $p(H \mid D)$ and $p(\sim H \mid D)$ together in sequence; that is, where, for example, the following goal statement is added to the Glom-Fizo task presented above, 
[35f] Estimate the chances in 100 that this creature is a Fizo.

The representation of the task would then be,

$$
\begin{aligned}
& \mathscr{S}_{1}=\{\text { Glom }\} \\
& n\left(\mathscr{S}_{1}\right)=1 \text { million } \\
& \mathrm{S}_{1}=\{\text { Glom } \wedge \text { play harmonica }\} \\
& p\left(S_{1} \bar{T} \mathscr{S}_{1}\right)=98 \% \\
& \mathscr{S}_{2}=\{\text { Fizo }\} \\
& \begin{array}{r}
n\left(\mathscr{S}_{2}\right)=1 \text { million } \\
S_{2}=\{\text { Fizo } \wedge \text { play_harmonica }\} \\
p\left(S_{2} \mid \mathscr{S}_{2}\right)=58 \%
\end{array} \\
& \text { — creature } \wedge \text { play_harmonica } \wedge \text { [Glom, } \sqcup \%] \\
& \text { — creature } \wedge \text { play_harmonica } \wedge[\text { Fizo, } \sqcup \%]
\end{aligned}
$$

In this case, individuals who realise that the element defined by the statement [35d] can either be a Glom or a Fizo no longer need to update their representation of the goal statement as this possibility is already represented by representation $(35 \mathrm{~d}, \mathrm{f})$. In other words, the presence of the additional goal statement explicitly represents the implicit disjunction in representation $\left(35^{*} \mathrm{~d}\right)$. Accordingly, even individuals who would have spontaneously inferred that the creature may either be a Glom or a Fizo in a single-goal task, no longer need to update their representation to account for this assumption in the complementary-goal task. Instead they can provide an answer to the first goal statement based on the probability associated with sample $S_{1}$ (thereby committing the inverse fallacy), and they can answer the second goal statement [35f, e] on the basis of the probability associated with sample $S_{2}$. Thus, on the basis of the propositions of SSR theory, one can explain why the presence of two complementary goal statements in Experiment 3 led to an increase in the proportion of participants committing the inverse fallacy. Namely, the proportion of participants committing the inverse fallacy will be greater when the task presents two complementary goal statements because even participants who would have aggregated both $p\left(S_{1} \mid \mathscr{S}_{1}\right)$ and $p\left(S_{2} \mid \mathscr{S}_{2}\right)$ when the task only presents one goal statement would now be expected to commit the inverse fallacy.

This result is summarised by the following corollary: 
Corollary 3. The inverse fallacy, or the tendency to evaluate the posterior probability based on its corresponding inverse likelihood, will be more prevalent when the task's goal statement is in a elementary form and asks for both $p(H \mid D)$ and $p(\sim H \mid D)$ in lieu of $p(H \mid D)$ only.

The results of Experiments 3 and 4 provide empirical verification for this corollary. This also further illustrates how SSR theory represents an extension of MMT. Whereas corollary 3 can be derived from the propositions of SSR, it does not necessarily follow from the principles of the MMT of naïve probabilistic reasoning.

\section{Bayesian outcomes, natural frequencies and chance formats}

In chapter VI, both a natural frequency and a chance set-based task were used. With both tasks, the modal judgement output elicited coincided with the Bayesian criterion. Once again, SSR theory can account for these results. The statements of the set-based task in natural frequency format used in Experiment 5 (see chap. VI) can be summarised as follows,

[36a] 30 out of every 100 individuals who apply for a position will get it.

[36b] 24 out of the 30 applicants who obtained the position they applied for, had also passed the psychometric tests.

[36c] However, 35 out of the remaining 70 applicants who failed to get a position also had passed the psychometric tests.

[36d] Consider the case of individuals who apply now. Among those who will pass the psychometric tests, how many will actually obtain the position they applied for? applicants out of applicants.

This task should lead to the following representation,

$$
\begin{gathered}
\mathscr{S}_{1}=\{\text { individual } \wedge \text { applied }\} \\
S_{1}=\{\text { individual } \wedge \text { applied } \wedge \text { get_position }\} \\
n\left(S_{1}\right)=30 \\
S_{2}=\{\text { individual } \wedge \text { applied } \wedge \text { get_position } \wedge \text { passed_tests }\} \\
n\left(S_{2}\right)=24 \\
\neg S_{1}=\left\{\text { individual } \wedge \text { applied } \wedge \begin{array}{c}
\neg \text { get_position }\} \\
n\left(\neg S_{1}\right)=70
\end{array}\right. \\
S_{3}=\{\text { individual } \wedge \text { applied } \wedge \neg \text { get_position } \wedge \text { passed_tests }\} \\
n\left(S_{3}\right)=35
\end{gathered}
$$

$$
\begin{gathered}
\text { \{individual } \wedge \text { applied } \wedge \text { passed_tests }\}=\mathscr{S} \\
\therefore \mathscr{S}=S_{2} \cup S_{3} \\
\{\text { individual } \wedge \text { applied } \wedge \text { passed_tests } \wedge \text { get_position }\}=S \\
\therefore S=S_{2} \\
n(S) \text { out of } n(\mathscr{S})=\sqcup \text { out of } \sqcup \\
\therefore n\left(S_{2}\right) \text { out of } n\left(S_{2}\right)+n\left(S_{3}\right)=24 \text { out of } 59
\end{gathered}
$$

Statement [36b] defines a reference set $\mathscr{S}_{2}$ such that $\mathscr{S}_{2}=$ individual $\wedge$ applied $\wedge$ obtain position\}. Assuming that judges confounded intensions 'get position' and 
'obtain_position'; $\mathscr{S}_{2}$ can be identified with sample $S_{1}$ as the numerical, intensional and determinative cues were all satisfied. It follows that the representation of subset $S_{2}$ defined by $S_{2}=\{$ individual $\wedge$ applied $\wedge$ get_position $\wedge$ passed_tests $\}, n\left(S_{2}\right)=24$ could be included in $S_{1}$ (Propositions 2a, 2c). Statement [36c] defined the reference set $\mathscr{S}_{3}$ such that $\mathscr{S}_{3}=\{$ individual $\wedge$ failed_get_position $\}$. Even though there was no sample explicitly matching the intensions of $\mathscr{S}_{3}$, the numerical and determinative cues in this statement pointed to the complement of set $S_{1}$ within $\mathscr{S}_{1}$, namely, $\neg S_{1}=$ individual $\wedge$ applied $\wedge$ $\neg$ get_position $\}, n\left(\neg S_{1}\right)=70$. As a result, the subset $S_{3}$ defined by $S_{3}=$ individual $\wedge$ applied $\wedge \neg$ get_position $\wedge$ passed_tests $\}, n\left(S_{3}\right)=35$ could be included in $\neg S_{1}$ (Propositions 2a, 2c).

The goal statement was a set-statement asking for the number of elements in the subset $S$ defined by $S=\{$ individual $\wedge$ applied $\wedge$ passed_tests $\wedge$ obtained_position $\}$ out of the number of elements in the reference set $\mathscr{S}$ defined by $\mathscr{S}=$ individual $\wedge$ applied $\wedge$ passed_tests $\}$. There is only one sample within the representation that can be identified with $S$, namely $S_{2}$ (still assuming that judges confounded intensions 'get_position' and 'obtained_position'). The goal statement's reference set $\mathscr{S}$ encompasses two existing samples, namely $S_{2}$ and $S_{3}$. Thus, the modal answer predicted by SSR theory is $n\left(S_{2}\right)$ out of $n\left(S_{2}\right)+n\left(S_{3}\right)$ (Proposition 3a, 3d); that is, 24 out of 59, which corresponds to the Bayesian criterion and the modal answer actually observed in this task (see chap. VI, p. 176).

In the same experiment, the Set-based task presented in an absolute chance format read as follows:

[37a] Individuals who apply for a position have 30 chances out of 100 of getting it.

[37b] 24 of the 30 chances of getting the position are associated with a pass in the psychometric tests.

[37c] However, 35 out of the remaining 70 chances of failing to get a position are also associated with a pass in the psychometric tests.

[37d] Consider the case of a person who applies now. Among his or her chances of passing the psychometric tests, how many chances will be associated with a successful appointment? chances out of chances.

The representation of this task is as follows, 


$$
\begin{aligned}
& \mathscr{S}_{1}=\{\text { individual } \wedge \text { applied }\} \\
& \mathrm{S}_{1}=\{\text { individual } \wedge \text { applied } \wedge \text { get_position }\} \\
& p\left(S_{1} \mid \mathscr{S}_{1}\right)=30 \text { out of } 100 \\
& \text { or } \\
& { }_{\circ} \text { _individual } \wedge \text { applied } \wedge \text { [get_position, } 30 \text { out of 100] } \\
& \mathscr{T}_{2}=\{\text { chance } \wedge \text { get_position }\} \\
& S_{2}=\{\text { chance } \wedge \text { get position } \wedge \text { pass }\} \quad n\left(\mathscr{S}_{2}\right)=30 \\
& n\left(\mathrm{~S}_{2}\right)=24 \text { an } \\
& \mathscr{S}_{3}=\{\text { chance } \wedge \text { fail_get_position }\}
\end{aligned}
$$

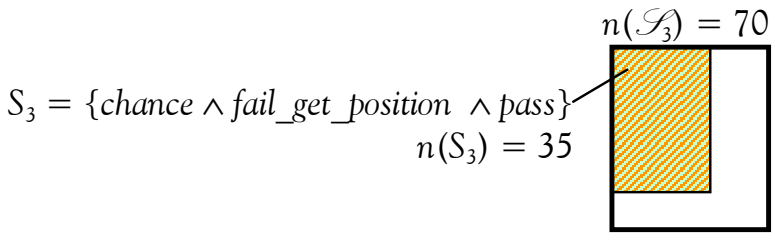

$$
\begin{aligned}
& \mathscr{S}_{4}=\mathscr{S}_{2} \cup \mathscr{S}_{3}
\end{aligned}
$$

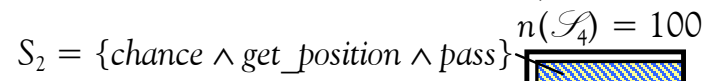

$$
\begin{aligned}
& n\left(S_{2}\right)=24 \\
& S_{3}=\{\text { chance } \wedge \text { fail_get_position } \wedge \text { pass }\} \\
& n\left(S_{3}\right)=35 \\
& \{\text { chance } \wedge \text { pass }\}=\mathscr{S} \\
& \therefore \mathscr{S}=S_{2} \cup S_{3} \\
& \text { \{chance } \wedge \text { pass } \wedge \text { get position }\}=S \\
& \therefore S=S_{2} \\
& n(S) \text { out of } n(\mathscr{Y})=\sqcup \text { out of } \sqcup \\
& \therefore n\left(S_{3}\right) \text { out of } n\left(S_{2}\right)+n\left(S_{3}\right)=24 \text { out of } 35
\end{aligned}
$$

Statement [37a] is ambiguous because although it refers to a set of individuals, the probability information applies to each individual rather than the set. Judges may interpret this statement as either a set statement [i.e., $\mathscr{S}_{1}=\{$ individual $\wedge$ applied $\}, S_{1}=$ \{individual $\wedge$ applied $\wedge$ get_position\}, where $p\left(S_{1} \mid \mathscr{S}_{1}\right)=30$ out of 100] or as an elementary statement (i.e., $\forall x$, individual $\wedge$ applied, [get_position, 30 out of 100]) where the mention $\forall x$ reads 'for all $x$ '. There is no such ambiguity in statement [37b]. It clearly defines the reference set $\mathscr{S}_{2}$ as $\mathscr{T}_{2}=$ \{chance $\wedge$ get position $\}, n\left(\mathscr{S}_{2}\right)=30$ and its subset $S_{2}=\{$ chance $\wedge$ get_position $\wedge$ pass $\}, n\left(S_{2}\right)=24$. Note that $\mathscr{T}_{2}$ can not be assimilated with an existing sample because of an intensional conflict, namely, the statement [37b] refer to elements characterised by intension 'is a person' whereas statement [37a] refers 
to elements characterised by intension 'is a chance.' In this particular case, it seems rather unlikely that judges will assimilate these two intensions, and $\mathscr{S}_{2}$ will be represented as another sample space (Propositions 2a, 2c). Statement [37c] defines the reference set $\mathscr{S}_{3}$ as $\mathscr{S}_{3}=\{$ chance $\wedge$ fail_get_position $\}, n\left(\mathscr{S}_{3}\right)=70$ and its subset $S_{3}=$ \{chance $\wedge$ fail_get_position $\wedge$ pass $\}, n\left(S_{3}\right)=35$. Again, there is no available sample with which $\mathscr{S}_{3}$ can be assimilated, and its representation would therefore entail the representation of a new sample space (Propositions 2a, 2c).

The goal statement asks for the number of elements in the subset $S=\{$ chance $\wedge$ pass $\wedge$ successful_appointment $\}$ out of the number of elements in the reference set $\mathscr{S}=$ \{chance $\wedge$ pass\}. Assuming that individuals will confound intensions 'successful_appointment' and 'get_position,' $\mathscr{S}$ can be identified as the union of $S_{2}$ and $S_{3}$ and $\mathrm{S}$ can be identified as $\mathrm{S}_{2}$. The combination of existing sample spaces $\mathscr{S}_{2}$ and $\mathscr{S}_{3}$ leads to the evaluation of $n\left(S_{2}\right)$ out of $n\left(S_{2}\right)+n\left(S_{3}\right)$ (Proposition 3a, 3e); that is, 24 out of 59, which once more corresponds to the Bayesian criterion and the modal answer actually observed in this task (see chap. VI, p. 176)

Thus, the application of the propositions of SSR theory to the Set-based tasks used in Experiment 5 can account for the Bayesian outcomes observed. This confirms the generalisability of the propositions of SSR theory which can be insightfully applied to virtually any posterior probability judgement task. This analysis provides interesting insights into the processes underlying those outcomes. In both tasks, the use of synonymous intensions may have increased interpretational ambiguity and perhaps cognitive effort (assuming that inferring that two different intensions describe a unique feature involves more cognitive processing than identifying to identical intensions). Although the tasks were originally assumed to differ only with regards to the probability formats used (i.e., natural frequency vs, absolute chance), the application of the propositions of SSR theory to the statements used revealed that the representations elicited by these two tasks differed quite dramatically. For instance, whereas both tasks elicited normative outcomes, they entailed different sample space representations with varying numbers of intensions defining samples. For instance, although the natural frequency task entailed a fully inclusive representation, its samples could be described with up to 4 different intensions. By contrast, although the absolute chance task relied on multiple samples, its samples were defined with at most 3 different intensions. The fact that a task based on multiple samples still elicited Bayesian outcomes stresses the 
limitations of these tasks to study Bayesian reasoning: even without assuming that the likelihood information was conditioned on the base-rate information (which is a necessary assumption to warrant the normative status of the computation), individuals could compute an answer that coincided with the Bayesian criterion. A more controlled variation of these features may help distinguish the actual effect of differing probability format from the effect of processing efforts on the proportion of normative outcomes observed.

\section{CONCLUSIONS}

The present chapter introduced a new algorithmic account of posterior probability judgements, called Sample Space Representation theory. SSR theory proposes that there are three processing stages involved in posterior probability judgement tasks issued from the Textbook-problem paradigm and the Set-based paradigm. Namely, first, an initial representation is built, based on the first probability statement of the task. Secondly, the subsequent statements are processed and the initial representation is updated. Finally, the outcome judgement is made, on the basis of the final representation of the task. The set of propositions of Sample Space Representation theory are summarised in Table VII1. These propositions define the exact processes underlying each of the three stages involved, from the ways in which different task statements will elicit different representations, to the ways in which different goal statements will entail different judgemental methods to be elicited, and hence yield different judgement outcomes. 


\section{Table VII-1}

Overview of the propositions of Sample Space Representation theory.

\section{Stage 1 - Initial sample space representation}

Proposition $19 \quad$ The structure and content of the initial sample space representation are determined by the type (elementary vs. sample) of a given statement and its intensions (i.e., the specific descriptive units defining its components).

Proposition $1 b \quad$ The element defined by an elementary statement is represented by a single element, characterised by the intension(s) defined in the statement, and (if applicable) the probability associated with those intension(s).

Proposition 1c The reference set defined by a set statement is represented by a sample space of elements. When the set statement also refers to a subset of the reference set, this subset is represented by a sample included in the sample space. Both sample space and sample are characterised by their respective intension(s), as defined in the set statement, and (if applicable), by either the probability of the sample in reference to the sample space or the respective sizes of sample and sample space.

\section{Stage 2 - Updating the representation}

Proposition $2 a$ If the task contains more than one statement, the existing representation will be updated as the judge processes more information.

Proposition $2 b \quad$ Provided that an element $x_{i}$ has been defined by a previous elementary statement, its intensions will be updated to include the intensions defined in a new elementary statement if the following conditions are satisfied:

(i) There is an intensional cue: The intensions of the element $x_{i+1}$ match those of element $x_{i}$, thereby indicating that $x_{i+1}$ is an element similar to $x_{i}$.

(ii) There is a determinative cue: A definite determiner is used to indicate that the element $x_{i+1}$ is the same element as the $x_{i}$ element.

Proposition $2 c \quad$ Provided that the representation of a sample space $\mathscr{S}_{i}$ has been elicited by a previous set statement, the representation of a new set $S_{j+1}$ will be included in $S_{i}$ or in $S_{i j}$, a sample already included in $S_{i}$, if the following conditions are satisfied:

(i) There is an intensional cue: The intensions of the reference set of $S_{j+1}$ either match the intensions of $\mathscr{S}_{i}$ or those of $S_{j}$, thereby indicating that the representation of $S_{j+1}$ is to be included in $\mathscr{S}_{i}$ or in $S_{j}$, respectively.

(ii) There is a numerical cue: The size of the reference set of $S_{j+1}$ is either known to be equal to the size of the sample within which it is to be included (absolute formats) or the size of $S_{j+1}$ can be made relative to the size of this sample (relative formats).

(iii) There is a determinative cue: A definite determiner is used to indicate that the elements of the reference set of $S_{j+1}$ are the same as the elements of the sample within which the representation of $S_{j+1}$ is to be included.

\section{Stage 3 - Final representation and judgement}

Proposition $3 a \quad$ Individuals will review their representation of the task's premises and form their probability judgement on the basis of the samples or elements whose intensions match the goal statement to the highest degree.

Proposition $3 b \quad$ Individuals may alter their representation of what is explicit in the statements, either to represent additional assumptions or to improve matching of the number of the samples or elements whose intensions match those of the goal statement.

Proposition $3 c \quad$ If the goal statement is elementary, individuals will review their representation and will base their judgement on the probability associated with the element or sample having the highest number of intensions matching those of the goal statement. If there is more than one element or sample thus identified, individuals will produce a judgement based on an aggregate of the probabilities associated with these elements and/or samples. The exact modalities of the aggregation (i.e., anchor and adjustment, multiplication, addition, etc.) will depend on individual abilities and preferences.

Proposition 3d If the goal statement is a set statement, individuals will evaluate the proportion of elements in the sample(s) whose intension(s) match those of the subset defined in the goal statement relative to the proportion of elements in the sample(s) whose intension(s) match those of the reference set of the goal statement.

Proposition $3 e \quad$ If the proportions highlighted by the set goal statement are relative to different samples within a unique sample space, individuals will first relate these proportions to this sample space. If those proportions are relative to different sample spaces, individuals will either assimilate (i.e., consider that $\mathscr{S}=\mathscr{S}_{\mathrm{i}}=\mathscr{S}_{\mathrm{j}}$ where $\mathscr{S}_{\mathrm{i}}$ and $\mathscr{S}_{\mathrm{j}}$ are the sample spaces that include the samples highlighted by the goal statement) or combine the different samples spaces (i.e., consider that $\mathscr{S}=\mathscr{S}_{\mathrm{i}} \cup \mathscr{S}_{\mathrm{j}}$ ). 
Table VII-2

Overview of the corollaries derived from the propositions of Sample Space Representation theory

A posterior probability judgement task will elicit a fully inclusive representation if:
(i) The base rate statement defines a reference set $S$ and two exclusive and mutually exhaustive
subsets, $H_{1}$ and $H_{0}$.
(ii) Intensional cue: The likelihood statements expressing $p\left(D \mid H_{1}\right)$ and $p\left(D \mid H_{0}\right)$ define reference
sets whose intensions match the intensions of $H_{1}$ and $H_{0}$, respectively.
(iii) Numerical cue: The likelihood statements for $p\left(D \mid H_{1}\right)$ and $p\left(D \mid H_{0}\right)$ respectively define
subsets $D \cap H_{1}$ and $D \cap H_{0}$ whose sizes can be made relative to the size of $H_{1}$ and $H_{0}$,
respectively.
(iv) Determinative cue: The elements of the reference sets defined by the likelihood statements for
$p\left(D \mid H_{1}\right)$ and $p\left(D \mid H_{0}\right)$ are indicated to be the elements of $H_{1}$ and $H_{0}$, respectively.
Participants' posterior probability judgement of $p(H \mid D)$ will be Bayesian if,
(i) The intensions of the task statements lead to a fully inclusive representation (see Corollary 1$)$.
(ii) The goal statement is a set statement.
(iii) The intension(s) of the reference set defined in the goal statement can be matched to the
intension(s) of the sample $H$ whose representation was elicited by the base rate statement of
the task (intensional cue) and the elements of this reference set are indicated to be the
elements of $H$ (determinative cue).
(iv) The intension(s) of the subset defined in the goal statement can be matched to the
intension(s) of the sample $D$ elicited by the likelihood statements of the task (intensional
cue) and the elements of this subset are indicated to be the elements of $D$ (determinative
cue).
(v) Individuals can calculate the size of $H$ relative to the size of sample $D$ (numerical cue).
The inverse fallacy, or the tendency to evaluate the posterior probability based on its
corresponding inverse likelihood, will be more prevalent when the task goal statement is in a
elementary form and ask for both $p(H \mid D)$ and $p(\sim H \mid D$ ) in lieu of $p(H \mid D)$ only.

Altogether, the propositions and corollaries of Sample Space Representation theory answer the criticisms made of earlier theoretical accounts of posterior probability judgements in these paradigms. Causality (Tversky \& Kahneman, 1980), specificity (Bar-Hillel, 1980) and partitive effects (Macchi, 1995, 2000) are all accounted for by SSR theory, which stands as a more formalised explanation of the processes underlying those effects than any of the existing accounts of algorithmic judgements in Textbookproblems. With regards to the Set-based paradigm, SSR theory suggests that the natural frequency information format is neither sufficient nor necessary to elicit Bayesian judgements. This information format is not sufficient to elicit Bayesian judgements because fully inclusive representations-which mediate the common elicitation of Bayesian judgements-are only warranted if the statements of the task feature certain intensional and determinative cues. It is not necessary because the numerical cue inherent to natural frequency format of information is also present in relative frequency formats. Additionally, Sample Space Representation theory not only overcomes the shortcomings associated with the application of MMT to posterior probability judgements (esp. in relation to the domain of application of the subset principle) but also predicts most Bayesian and non-Bayesian judgement outcomes observed in these tasks. 
Finally, the propositions of Sample Space Representation theory provide insight into the processes underlying empirical outcomes reported within this thesis. Namely, in relation to the Textbook-problem paradigm, the finding that the presentation of two elementary complementary goal statements increased the proportion of inverse fallacy judgements (see chap. V) could be accounted by the propositions of SSR theory. In relation to the Set-based paradigm, SSR theory could explain why the tasks used both elicited high rates of Bayesian performances, despite the fact that both tasks may have been based on slightly different representations.

The next chapter will conclude this thesis by summarising its main achievements as well as discussing its implications for future experimentation and theoretical developments, and outlining directions for future research. 
Let us, then, be up and doing,

With a heart for any fate;

Still achieving, still pursuing,

Learn to labour and to wait.

(Henry Wadsworth Longfellow, A Psalm of Life, 1838)

7 he set objective of this thesis was to develop the process-focused study of

posterior probability judgements within each of the three paradigms of research currently in use, namely the Social-judgement paradigm, the Textbookproblem paradigm and the Sample-based judgement paradigm. The characterisation of the process-focused perspective led to the development of a new agenda of research. Whereas most studies of posterior probability judgements have been concerned with the elicitation of Bayesian judgements, the purpose of the present research was to (i) achieve a systematic computational account of the judgement methods or strategies underlying observed judgement outputs, and (ii) use such computational accounts as a basis for developing an algorithmic account explaining how individuals' processing of the task led to the application of such methods. In the present chapter, the main achievements of this doctoral work with regards to these two objectives are summarised, the implications for future experimentation and theoretical development are discussed, and the directions for future research are outlined.

In chapter III, the existing body of literature on posterior probability judgements was reviewed and evaluated from a process-focused perspective. This review in turn highlighted new issues that had been overlooked in the predominant outcome-focused study of these judgements. It was acknowledged that these issues may not have been central to the outcome-focused approach, which focuses on the elicitation of normative judgements. They were, however, crucial to the development of the process-focused study of posterior probability judgements and, as such, formed the impetus for the empirical and theoretical research programme of the present thesis.

\section{COMPUTATIONAL ANALYSIS: \\ CAN ONE TELL WHAT JUDGEMENT METHODS UNDERLIE POSTERIOR PROBABILITY JUDGEMENTS?}

The primary (computational) objective of the process-focused study of posterior probability judgements was to identify what judgement methods were underlying 
observed judgement outputs. The review of the existing literature, within both the Social-judgement and the Textbook-problem experimental paradigms, revealed that descriptive accounts commonly focused on people's tendency to neglect base-rate information in these problems. The base-rate neglect account is a useful construct for an outcome-focused study of posterior probability judgements: it sets a straightforward agenda for the researcher who aims at eliciting normative judgements. Accordingly, research within these two paradigms was mainly concerned with the identification of the factors that may enhance base-rate utilisation. By contrast, however, it was contended that the base-rate neglect account did not satisfactorily address issues arising from a process-focused approach. Indeed, where the primary objective of this approach is to identify what people do to produce their judgement output, the base-rate neglect account is clearly inadequate: it merely suggests what people may not do.

The review of the literature led to the identification of alternative descriptive accounts. Within the Social-judgement paradigm, authors suggested that individuals relied on the representativeness heuristic whereas others suggested that people relied on the inverse fallacy. Within the Textbook-problem paradigm, results observed suggested that people commit the inverse fallacy, whereas the representativeness heuristic was de jure discarded as a possible descriptive account (see p. 58). The empirical work presented in chapters IV and V was aimed at testing whether these accounts could provide a valid description of the judgement methods underlying observed judgement outputs, within the Social-judgement paradigm and the Textbook-paradigm, respectively.

\section{On the Social-judgement paradigm}

Results from the first experiment conducted within the Social-judgement paradigm (see chap. IV) revealed no effect of the manipulation of the base-rate information on the judgement outputs observed. Although a significant, albeit small, effect of base-rate information is usually observed within this paradigm, it was acknowledged that the present result may have been due to the difference in statistical power between this experiment and experiments that had been previously reported within the literature. Nevertheless, it was found that average judgement outputs significantly differed from Bayesian prescriptions confirming that Bayes's theorem does not provide a good descriptive account of judgements within this paradigm. The novel contribution of this experiment was the test for the validity of the inverse fallacy account in describing 
judgement outputs. Results confirmed that judgements of the posterior probability $p(H \mid D)$ in both high and low base-rate conditions were confounded with judgements of the inverse likelihood $p(D \mid H)$, in the absence of any base-rate information.

It was argued, however, that such a nomothetic approach, based on average judgement outcomes, was not sufficient to establish whether each individual was effectively evaluating $p(D \mid H)$ when asked to evaluate $p(H \mid D)$. A second experiment was designed to address this shortcoming. This time, a within-subjects design was used and judgements were analysed using an idiographic approach. Such an approach consisted in testing, for each individual's series of posterior probability judgements, whether these judgements could be accounted for by different computational accounts (i.e., the inverse fallacy, the representativeness heuristic, the base-rate neglect hypothesis, or else, the Bayesian algorithm). Results showed that, whereas most individuals produced outputs that could not be predicted by any of these four descriptive accounts, some provided judgements that could be described by more than one account (e.g., representativeness heuristic, inverse fallacy, and base-rate neglect).

The results of this second experiment thus clearly illustrated the limits of an analysis of judgements based on averaged outputs, such as the analysis carried out in the first experiment. Indeed, results from Experiment 1 could have led to the erroneous conclusion that most individuals were committing the inverse fallacy. The findings of Experiment 2, however, revealed that this conclusion was no longer warranted when the analysis was based on individuals' series of judgements rather than averaged judgements. But although an idiographic approach may provide a better basis for identifying the strategies used by participants in making posterior probability judgements in Experiment 2, the fact that the different strategies tested rarely accounted well for the outputs observed suggests that numerical outputs alone may not be sufficient for unambiguously identifying the strategies used.

This failure to identify what strategies underpin the posterior probability judgements observed is nevertheless instructive: it has both theoretical and methodological implications. From a theoretical perspective, it shows that the widely held belief that people rely on the representativeness heuristic when making posterior probability judgements in Social-judgement tasks is not warranted. From a methodological perspective, these results call for the use of multiple methods to unambiguously identify the strategies used, or, at least, the use of a number of judgement outputs considerably higher than the usual series of five judgements, for a more general test of the extent to 
which representativeness judgements are better descriptors than inverse likelihood judgements.

The effort to establish a firm descriptive account also has consequences for normative issues. The collection of both $p(H \mid D)$ and $p(\sim H \mid D)$ judgements in the second experiment allowed for the test of the additivity principle at an individual level. Results revealed that although the sum of medians for complementary posterior probability judgements was generally close to 1 , only one out of the 16 participants who took part in the experiment systematically respected the additivity principle. This result has important methodological implications: if participants' complementary posterior probability judgements do not add up to one, then inferred $p(\sim H \mid D)$ estimates, evaluated from observed $p(H \mid D)$ judgements by subtracting their value from 1 , cannot be representative of the actual $p(\sim H \mid D)$ judgements that would have been observed if they had been elicited from the judges themselves. Yet it is not uncommon that analyses rely on such inferred estimates (e.g., Carroll \& Siegler, 1977; Gigerenzer et al., 1988; Schwartz et al., 1991; Wells \& Harvey, 1978), calling into question the descriptive validity of the results observed.

A possible explanation for observed deviations from additivity was suggested in Rottenstreich and Tversky's (1997) account of Support Theory. The authors indeed proposed that binary additivity would hold for judgements of $p(H)$ and $p(\neg H)$ but not for judgements of $p(H)$ and $p(\sim H)$ even if $\sim H$ corresponded to $\neg H$. For example, this implies that binary additivity would be expected to hold between judgements of the probability that a person is an engineer and that a person is not an engineer. By contrast, judgements of the probability that a person is an engineer and judgements of the probability that a person is a lawyer would not be expected to be additive, even when the sample space under consideration is defined to consist only of engineers and lawyers.

This would suggest that individuals who are asked to evaluate the probability that $x$ is an engineer rather than a lawyer, are in fact evaluating the probability that $x$ is an engineer rather than not an engineer. This argument was applied to posterior probability judgements observed in Social-judgement tasks (see chap. IV). It was demonstrated, however, that the assumption that $p(H \mid D)$ judgements were conditioned on the intersection of elements from both $H$ and $\neg H$, led to the proposition that observed $p(H \mid D)$ and $p(\sim H \mid D)$ should have been consistently superadditive (i.e., add to less 
than 1). This was a proposition that was not supported by the data observed, as in most cases, participants provided judgements that were either subadditive or superadditive.

\section{On the Textbook-problem paradigm}

The first experiment conducted within the Textbook-problem paradigm was aimed at distinguishing the inverse fallacy and the base-rate neglect accounts, in order to determine what judgement methods were underlying observed judgement outputs in Textbook problems. Results showed that roughly half of the sample of participants judging $p(H \mid D)$ consistently provided outputs that matched the inverse likelihood value, $p(D \mid H)$, in a series of 24 judgements. This result was observed despite the manipulation of the conditioning data or the target hypothesis asked: although participants were always provided with values for $p(D \mid H)$ and $p(D \mid \sim H)$, they estimated $p(H \mid D), p(\sim H \mid D), p(H \mid \sim D)$ and $p(\sim H \mid \sim D)$ to be equal to the values of $p(D \mid H)$, $p(D \mid \sim H), p(\sim D \mid H)$ and $p(\sim D \mid \sim H)$, respectively. In previous studies, the possibility that people may rely on an inverse likelihood method (i.e., commit the inverse fallacy) was only substantiated with the elicitation of a single judgement per participant, namely, $p(H \mid D)$ given the values of $p(H), p(\sim H), p(D \mid H)$, and $p(D \mid \sim H)$. In these situations, the fact that participants equated $p(H \mid D)$ with $p(D \mid H)$ could have resulted from a simple matching strategy (Evans, 1998) whereby participants simply 'picked' the value in the text whose description was closest to that of $p(H \mid D)$. Experiment 3, however, was the first experiment to demonstrate that a majority of individuals were not using such a matching heuristic, but instead were evaluating posterior probabilities with the value of their inverse likelihood, regardless of whether this value was directly available from the text.

This experiment also demonstrated that individuals were not 'merely' neglecting base-rate information. In the tasks given to participants, base-rates were set to be equal [i.e., $p(H)=p(\sim H)]$. In such cases, individuals whose only fallacy is to neglect base-rate information can be expected to exhibit judgement outputs that remain in line with Bayesian prescriptions. In contrast, the individual who evaluates posterior probabilities on the basis of their inverse likelihood would be expected to either overestimate or underestimate the Bayesian criterion, as a function of the direction of the deviation of the inverse likelihood value from the Bayesian criterion. Results indeed confirmed that participants' judgements consistently deviated from Bayesian prescriptions. This demonstrates that the base-rate neglect hypothesis was not sufficient to explain 
deviations from such prescriptions. Moreover, results showed that those observed deviations between judgement outputs and Bayesian criteria could be reliably predicted from deviations of inverse likelihood values from Bayesian criteria. This in turn confirms that the inverse fallacy, rather than the base-rate fallacy, could explain observed deviations from normative prescriptions.

With regards to the additivity issue, results also confirmed the importance of a precise knowledge of the methods underlying observed judgement outputs for understanding, as well as predicting deviations from the additivity principle. Indeed, from the assumption that participants were equating posterior probabilities and inverse likelihoods, it was possible to predict whether binary complementary posterior probability judgements would be subadditive or superadditive, as a function of the sum of their corresponding inverse likelihoods presented in the tasks. As for studies within the Social-judgement paradigm, there are significant methodological implications associated with the finding that participants generally do not respect the additivity principle in Textbook problems. Namely, this raises the question of the validity of results based on recoding procedures where $p(\sim H \mid D)$ judgements are recoded as $1-p(\sim H \mid D)$ before being assimilated with $p(H \mid D)$ judgements (e.g., see Slowiaczek et al., 1992, Experiment 1A). Since binary complementary judgements are not additive, i.e., $p(H \mid D)$ and $p(\sim H \mid D)$ judgements do not add up to one, then the $p(H \mid D)$ judgement value cannot be accurately estimated from one minus the value of the $p(\sim H \mid D)$ judgement.

From a theoretical perspective, the fact that the direction and the magnitude of the deviations from additivity of binary complementary posterior probability judgements could be accurately predicted from the knowledge of inverse likelihoods has important consequences for Support theory (Rottenstreich \& Tversky, 1997; see also, Tversky \& Koehler, 1994). In contrast with the designer-accountant task in the Social-judgement paradigm, the use of unnatural complementary categories in Experiment 3 prevented the possibility that participants would rely on a different sample space than that defined in the task. Indeed, whereas in the designer-accountant task, participants may have used a sample space including professionals that were neither designers nor accountants, the use of unnatural categories in the glom-fizo task allowed to control for such inferences as it was not possible for participants to consider a sample space including creatures that were neither 'gloms' nor 'fizos.' Still, contrary to what is suggested by Support theory, the results observed in this task demonstrated that binary complementary judgements were not additive. Interestingly, from a rationalist 
perspective, these results should not lead to the conclusion that participants do not know that complementary judgements should be additive. When participants were asked to estimate $p(H \mid \sim D)$, those who committed the inverse fallacy did infer the value of $p(D \mid \sim H)$ by subtracting the value of $p(D \mid H)$ provided in the text from 1 . This shows that those participants knew that $p(D \mid H)+p(\sim D \mid H)=1$. Anecdotally, when providing informal feedback on the task to participants who had provided non-additive posterior probability judgements, simply asking them to add up complementary estimates was sufficient to make them realise that their judgement method had been inappropriate. This suggests that they understood that those judgements should have additive but somehow failed to check that they provided additive judgements.

A noteworthy finding of Experiment 3 was also the fact that the proportion of inverse judgements observed was significantly higher than what is usually observed in Textbook problems, whereas the proportion of Bayesian judgements observed was significantly lower. It was conjectured that the presence of two goal statements together in sequence [namely, the prompt for a $p(H \mid D)$ judgement and that for a $p(\sim H \mid D)$ judgements] had led to these observations. Accordingly, it was hypothesized that participants who were only asked to estimate $p(H \mid D)$ or $p(\sim H \mid D)$ but not both, may be induced to aggregate both $p(D \mid H)$ and $p(D \mid \sim H)$ to form their judgement, thereby reducing the proportion of inverse fallacies as well as increasing the proportion of Bayesian judgements observed. This hypothesis was verified in Experiment 4, where the removal of the complementary task goal was sufficient to significantly decrease the proportion of inverse judgements observed, as well as significantly increase the proportion of Bayesian judgements observed.

No available theoretical accounts for the cognitive processes at stake in Textbook problems (Bar-Hillel, 1980; Macchi, 1995, 2000; Tversky \& Kahneman, 1980) could provide a satisfactory account of these results. By constrast, the Sample Space Representation theory introduced in the penultimate chapter of this thesis was thereafter shown to provide such an account. It not only explained the general modalities by which the representation of the task determined the judgement method applied to the task, but also predicted how seemingly inconsequential task features, such as whether participants are asked to evaluate binary complementary posterior probability judgements together in sequence, can determine what type of judgement method will be applied to the task. 


\section{On the Set-based judgement paradigm}

The Set-based judgement paradigm is the latest of the three experimental paradigms within which posterior probability judgements have been investigated. As for the two preceding experimental chapters of this thesis, the undertaking of a process-focused perspective led to the identification of novel research issues. Whereas conflicting descriptive accounts of the judgement methods underlying observed outputs existed within the Social-judgement paradigm, and the Textbook-problem paradigm, this was no longer true for the Set-based paradigm. Within this paradigm, it is now accepted that a majority of judgement outputs result from the application of a Bayesian method, provided that they are elicited with a fully inclusive task; that is, a task which defines a unique reference set divided into exhaustive subsets, and presents a goal statement that focuses on the proportion of elements in the set $H \cap D$ relative to the set $D$.

These observations were taken as evidence for human Bayesian reasoning (e.g., see Gigerenzer \& Hoffrage, 1995). It was contended herein, however, that the application of a Bayesian algorithm, i.e., the appropriate evaluation of the number of elements in the set $D \cap H$ relative to the number of elements in the set $D$, did not necessarily entail Bayesian reasoning, per se. Bayesian reasoning was defined as the inference that such a ratio must be evaluated in order to estimate the posterior probability $p(H \mid D)$. In the last study of this thesis, an experiment was designed to investigate whether individuals who exhibited judgement outputs that coincided with the Bayesian criterion would also identify the appropriate strategy for accurately evaluating $p(H \mid D)$ in a general reasoning task. Although results showed a significant association between Bayesian outputs and Bayesian reasoning choices, this was rather small in size. It was found that only half the individuals who provided a Bayesian judgement output, in fully inclusive posterior probability judgement tasks, also correctly inferred that, in order to evaluate $p(H \mid D)$, they needed to evaluate the number of elements in the set $D \cap H$ out of the number of elements in the set $D$.

This result in turn suggests that the assumption that the observation of Bayesian judgement provides evidence for Bayesian reasoning is not warranted. Further research is still needed, however, to substantiate the results observed. The reasoning task used to provide evidence for Bayesian reasoning presented participants with an array of reasoning methods, all based on the evaluation of different ratios of absolute units (whether natural frequencies or partitioned chances). It is possible that the presence of 
an array of choices influenced individuals' responses. Additionally, this method constrained individuals to rely on numerical information (i.e., natural frequencies or partitioned chances) to estimate $p(H \mid D)$, whereas it is possible that more 'natural' reasoning processes may rely on subjective arguments such as pro and con arguments to infer the posterior probabilities (e.g., see Fox, 1994). Thus, a more direct test of whether individuals would rely on a ratio of instances to estimate a single-event probability would investigate whether participants can infer they need to evaluate such a ratio, without prompting the use of frequencies or chances of observations, and in the absence of reasoning method exemplars.

With regards to posterior probability judgements, the results also showed that the proportion of judgements whose output coincided with the Bayesian criterion was not affected by the type of information (natural frequency vs. partitioned chance), thereby replicating findings observed by Girotto and Gonzalez (2001) which demonstrated that natural frequency formats were not necessary to elicit Bayesian judgments. Additionally, the elicitation of interpretations for a single-event probability statement revealed that natural frequencies were not a more natural format of interpretation. This is in contrast to what may have been expected from the hypothesis that humans are more adapted to process probabilities based on natural frequencies acquired by natural sampling rather than to probabilities or relative frequency (Gigerenzer \& Hoffrage, 1995). Contrary to what had been conjectured, however, the type and format of the interpretation provided by participants was found to be independent from their choice of reasoning method (Bayesian vs. non-Bayesian). A possible explanation for these results was that the type and format of the interpretations elicited may have been related to people's background knowledge rather than their ability to relate single-event probabilities with ratios of frequencies (see Harries \& Harvey, 2000).

\section{Synthesis}

The process-focused approach characterized at the outset of this thesis provided the rationale for bringing together the three experimental paradigms within which posterior probability judgements are currently investigated. The experimental work presented both addressed and raised issues regarding the development of a systematic description of the judgement methods underlying observed judgement outputs, which defines the primary aim of the process-focused approach. Results observed within the Socialjudgement paradigm showed that still more research is needed to uncover the 
judgement methods elicited by these tasks. By contrast, results observed within both the Textbook-problem and the Set-based judgement paradigms unambiguously identified the main judgement methods elicited in these respective paradigms. The main judgement method elicited within the Textbook-problem paradigm was identified as the inverse method, commonly characterised as the 'inverse fallacy' whereby the posterior probability is judged to be equal to the value of its corresponding inverse likelihood. Within the Set-based judgement paradigm, the main judgement method elicited is a Bayesian method, whereby judges evaluate the number of elements in the set $D \cap H$ relative to the number of elements in the set $D$.

The issues regarding the conception of probability that may underlie judgements raised in Chapter II were not directly addressed in the work presented in all experimental paradigms reviewed in the present thesis. It seems reasonable, however, to consider that judgements in both Textbook-paradigm and Set-based paradigms were generally based on a ' $\psi \mu$ ' concept of probability (Vail, 1954; see chap. II); that is, a mathematical quantity computed on the basis of the quantitative information presented in the text, rather than on $\psi f$, which would be more readily invoked when participants are directly exposed to the occurrence of a repetitive event. It is possible, however, that some of the unexplained judgement outputs observed were based on a concept $\psi \beta$ whereby the final output was influenced by both the quantitative information provided in the text and, perhaps, other social-cultural beliefs. Similarly, within the Socialjudgement paradigm, it is quite possible that the variety of judgement outputs observed relied on an evaluation of $\psi \beta$ whereby individuals may have used both information about the relative frequency of occurrence of the events judged and socio-cultural information related to the social categories involved.

Thus, in order to gain a wider understanding of the range of processes underlying the different posterior probability judgements observed in a given task, a useful path to follow may be to investigate the type of probability concept that underlies those judgements. For example, verbal protocols could be used to explore what type of uncertainty is assessed by individuals when making posterior probability judgements within the different paradigms. The different existing taxonomies (Howell \& Burnett, 1978; Kahneman \& Tversky, 1982/1982d; Teigen, 1994; Vail, 1954) would allow for a 'top-down' approach to the investigation of psychological probability concepts, whereby 
resulting protocols could be content-analysed using categories derived from these taxonomies (Wilkinson, 2003).

The development of a reliable identification of the behaviours elicited by a particular task, however, is not the sole aim of the process-focused approach. Instead, such a descriptive account should form the basis for theoretical advance in the formalisation of the cognitive processes that underpin the behaviours thus identified. Therefore, with specific regards to posterior probability judgements, the ultimate aim of a processfocused study was to develop a theoretical account of the cognitive processes underpinning the elicitation of judgement methods identified. The second section of this chapter reviews the achievements and remaining issues to be addressed with regards to our theoretical understanding of the cognitive processes that underlie posterior probability judgements.

\author{
ALGORITHMIC ANALYSIS: \\ CAN WE TELL HOW DIFFERENT POSTERIOR PROBABILITY \\ JUDGEMENT TASKS ELICIT DIFFERENT JUDGEMENT METHODS?
}

\title{
Shortcomings of existing accounts
}

The review of existing algorithmic accounts of posterior probability judgement revealed that it was generally proposed that the judgement methods used by individuals to produce their judgement output could be determined by the interpretation of the general orientation of the task or by its mental representation.

Within the Social-judgement paradigm, algorithmic accounts of posterior probability judgements (e.g., Gigerenzer et al., 1988; Ginossar \& Trope, 1980, 1987) emphasized the role of the task orientation inferred (statistical vs. clinical) as well as its mental representation in the elicitation of judgement methods. These accounts, however, neither specified the ways in which the task may actually be represented nor how a particular representation would elicit the use of a particular judgement method. From a process-focused approach, theoretical advance in the understanding of the cognitive processes determining the application of a certain judgement method first requires the unambiguous identification of the method applied. It was contended in this thesis that traditional nomothetic approaches, based on averaged judgement outputs, did not satisfactorily fulfil this requirement. The empirical study of individual judgement methods underlying observed outputs was, however, inconclusive: it was not possible to unambiguously identify what judgement methods were elicited in Social-judgement 
tasks. Yet the development of a sound computational account of judgements in Socialjudgement tasks is a necessary step for validating the psychological plausibility of existing algorithmic accounts. At present, algorithmic accounts purporting to explain the origin of computational processes elicited by Social-judgement tasks only rely on aggregated outcomes and assume that individuals use the representativeness heuristic to produce their judgement outcome (Doherty et al., 1999; Gigerenzer et al., 1988; Ginossar \& Trope, 1980, 1987).

Existing accounts within the Textbook-problem paradigm stressed the importance of the interpretation of the probabilistic information as well as the role of the structure of the task in determining what pieces of information would be aggregated to produce the output of the posterior probability judgement (e.g., Bar-Hillel, 1980; Macchi, 1995; Tversky \& Kahneman, 1980). Once again, however, these accounts did not specify the exact modalities by which those interpretational or representational cues may mediate the elicitation of particular judgement methods.

Finally, with regards to the Set-based judgement paradigm, the main emphasis of existing theoretical accounts has been the role of the mental representation of the tasks presented in this paradigm. These accounts have generally suffered from the same shortcoming; namely, the provision of vague characterisations of the cognitive processes at stake in these tasks. One notable exception, however, was Johnson-Laird et al.'s (1999) account of probabilistic reasoning based on Mental Model Theory (JohnsonLaird, 1983). This account was the first to propose a detailed, process-focused, theory of the cognitive processes that may underlie probability judgements. The application of MMT to probabilistic reasoning provides a compelling explanation for the mechanisms underpinning probability judgements based on discrete possibilities. It was contended, however, that its application to posterior probability judgements was not without shortcomings. From an empirical perspective, it was noted that the finding that relative frequency formats could elicit a large proportion of Bayesian outputs was at odds with the theory's subset principle, which proposed that the observation of Bayesian outputs would be limited to tasks presenting information in a partitioned absolute format (whether based on frequencies or chances of occurrence). From a theoretical perspective, the principles of the theory did not explain why individuals did not apply the subset principle in standard Textbook-problems. 


\section{Sample Space Representation theory}

Based on the empirical work presented in this thesis, and existing empirical findings, a new general theoretical account, the Sample Space Representation theory (SSR), was introduced. According to SSR theory, the cognitive processes involved in Textbook and Set-based posterior probability tasks involve three main stages: the construction of the initial representation, the updating of the initial representation, and the application of the judgement method to the final representation. The theory proposes that the outcome of each of these three processing stages will be mediated by the type and intension of the statements upon which they are based. Whereas MMT suggests that all representations are based on an instantiation of individual elements, the SSR theory allows for the representation of samples, when such a representation is instigated by a statement referring to multiple elements. The SSR theory also formalises how the type of goal statement will determine the judgement method applied to the representation of the task. Another distinctive feature of the theory is the proposed crucial role of statements' intensions (i.e., the specific labelling of the elements characterised in these statements). In particular, SSR theory proposes that intensions determine whether existing sample representations will include samples defined by subsequent task statements in the updating processing stage, and what aspects of the representation will be integrated in the judgement output in the judgement processing stage.

\section{Theoretical implications}

Altogether, the propositions of Sample Space Representation theory provide a significant advance in our understanding of the cognitive processes underpinning posterior probability judgements. The detailed yet wide-ranging propositions of the theory can be applied to any Textbook-problem or Set-based scenario to predict what judgement outputs would be more likely to be observed. As shown in chapter VII, the application of these propositions to existing tasks allows explaining, not only why the inverse judgement method prevails in Textbook problems, but also why some participants may still aggregate $p(D \mid H)$ and $p(D \mid \sim H)$ information in estimating $p(H \mid D)$ rather than relying solely on the value of the likelihood $p(D \mid H)$. These same general principles also enable the formal characterisation of the effects of the so-called manipulations of causality (Tversky \& Kahneman, 1980) or specificity (Bar-Hillel, 1980) on the proportion of inverse judgements observed. Futhermore, these propositions also explain why the elicitation of binary complementary posterior probability 
judgements together in sequence would lead to an increase of the proportion of judgements based on the inverse fallacy, thereby accounting for the results observed in Experiment 4 (see chap. V).

Finally, with regards to the Set-based paradigm, the SSR theory accounts for existing findings, including the fact that some Set-based tasks presenting the information in a relative frequency format may be successful in eliciting outputs that coincide with the Bayesian criterion. On the basis of the propositions of the theory, it was possible to explain why fully inclusive Set-based tasks may lead to the elicitation of Bayesian outcomes whereas multiple set-based tasks do not. The SSR theory thus overcomes the identified shortcomings of the application of MMT to posterior probability judgements, which did not account for this finding. The SSR theory also accounts for the fact that different judgement strategies operate in Textbook problems and Set-based tasks by formalising the different judgement methods expected to be elicited by elementary and set goal statements, respectively. In this respect, the SSR theory expands on MMT whose subset principle only accounts for judgements made in Set-based tasks where the probability information is presented in a partitioned, albeit absolute, format. In addition, the implications of SSR theory extend beyond the addressing of shortcomings of previous existing accounts, as new empirical predictions and research issues can be derived from its propositions.

\section{Implications for future research}

Concerning Bayesian performances, SSR theory defines factors that may mediate the construction of a fully inclusive representation of the task, which is in turn a precondition for the elicitation of Bayesian judgements. Thus, SSR theory suggests that the manipulation of numerical, intensional, and determinative cues in posterior probability judgement tasks may affect the proportion of Bayesian outputs elicited, independently of the format in which the information is elicited. Existing evidence for the differences in performances observed with fully inclusive and multiple set-based tasks already substantiates the role of numerical cues.

Further empirical research is needed, however, to establish the necessary and sufficient conditions for the construction of a fully inclusive representation of the task. These conditions may relate to the features of the task. For example, it is possible that ambiguous intensions will impinge on whether or not a fully inclusive representation is formed. If so, reducing or increasing ambiguity by manipulating the intensions of the sets 
defined by the task may mediate observed rates of Bayesian performance. Alternatively, the use of external representational aids may facilitate the elicitation of fully inclusive representations, and hence, the elicitation of Bayesian judgements. The extent to which fully inclusive representations will be elicited may also depend on individual interpretations and inferences with regards to the information presented in the task. For example, synonymous intensions may be assimilated, bearing consequences on whether or not subsequent sample representations will be included in the existing sample space representation. To further understand these processes, both the determinants and the effects of such individual differences need to be investigated empirically.

SSR theory also opens new paths for the investigation of the processes underlying posterior probability judgements that are not normative. Regarding tasks that include elementary statements (which cannot lead to fully inclusive representations and, as such, are unlikely to elicit Bayesian outputs), different interpretations are assumed to elicit different judgement outputs. For instance, it was suggested that an element characterised by intension $i$ with probability p, may be represented with intension $i$ alone or with the disjunction $i \vee \neg i$; the latter representation indicating the possibility that this element may in fact be described by $\neg i$. Depending on the representation favoured, SSR theory predicts that resulting $p(H \mid D)$ judgements will be based solely on $p(D \mid H)$ or on an aggregation of both $p(D \mid H)$ and $p(D \mid \sim H)$, respectively. The determinants of such differences in representations of otherwise identical statements of the task, however, remained to be established. Similarly, SSR theory proposes that multiple setbased tasks elicit judgements based on either the assimilation or the combination of the different samples represented. Here again, although the theory can predict differences in outputs given the method applied, the determinants for the application of these different strategies remain to be uncovered.

Finally, beside the importance of intensions in both task representation and judgement processes, another general issue highlighted by SSR theory is the role of implicit versus explicit negation in defining complementary hypotheses. Specifically, whether individuals will represent the intensions of two complementary hypotheses as two distinct intensions (i.e., $i_{1}$ and $i_{2}$ ) or as two complementary intensions (i.e., $i_{1}$ and $\neg i_{1}$ ) may have dramatic effects on their final judgements. Whereas explicit negations are likely to be represented as complementary intensions, this may or may not be the case for implicit negations. For example, would intensions 'black' and 'white' be naturally represented as distinct colours or complementary colours? This suggests that 
furthering our understanding of people's interpretation of negation would also help modelling the representations elicited by different tasks, and consequently improve predictions of judgement outputs in posterior probability judgement tasks.

\section{FinAL CONCLUSIONS}

The objective of this thesis was to develop our understanding of the processes underlying posterior probability judgements. This objective arose from my personal epistemological standpoint, namely the view that our understanding of behaviours needs to be anchored in descriptive rather than normative accounts. This objective echoed the recent call for the development and the test of theoretical accounts which explain how people behave rather than assess whether they fail to behave normatively (e.g., Hamm, 1998; Hertwig \& Todd, 2000; Krueger, 1998).

To achieve descriptive understanding, a framework of analysis, the process-focused approach, was developed and subsequently applied systematically to the study of posterior probability judgements. This framework, which paralleled Marr's (1982) distinction between computational and algorithmic levels of analysis of information processing systems, allowed both the transcending of the usual dichotomy between process-based and outcome-based methodologies, and the identifying of novel research issues in each of the three empirical paradigms currently used to study posterior probability judgements. By bringing together results from these three experimental paradigms, it was possible to shed new light on the judgement methods that may underlie posterior probability judgements. This in turn, allowed the development of a new algorithmic account, the Sample Space Representation theory, explaining how posterior probability judgements are made both within tasks pertaining to the Textbookproblem as well as the Set-based paradigms. 
Abelson, R. P., \& Levi, A. (1985). Decision making and decision theory. In G. Lindzey $\&$ E. Aronson (Eds.), The handbook of social psychology: Vol. 1. Theory and method. (pp. 231-309). New York: Random House.

Agresti, A. (1996). An introduction to categorical data analysis. New York: John Wiley \& Sons.

Ajzen, I. (1977). Intuitive theories of events and the effects of base rate information on prediction. Journal of Personality and Social Psychology, 35, 303-314.

Ajzen, I., \& Fishbein, M. (1975). A Bayesian analysis of attribution processes. Psychological Bulletin, 82, 261-277.

Ajzen, I., \& Fishbein, M. (1978). Use and misuse of Bayes' theorem in causal attribution: Don't attribute it to Ajzen and Fishbein either. Psychological-Bulletin, 85, $244-246$.

Arnauld, A., \& Nicole, P. (1992). La logique ou l'art de penser [Logic or the art of thinking]. Retrieved June 8, 2003, from http://gallica.bnf.fr/scripts/ConsultationTout.exe? $\mathrm{O}=\mathrm{N} 025788 \& \mathrm{E}=0$ (Original work published 1662)

Ayton, P. (1997). How to be incoherent and seductive: Bookmakers' odds and support theory. Organizational Behavior and Human Decision Processes, 72, 99-115.

Baratgin, J., \& Noveck, I. A. (2000). Not only base rates are neglected on the EngineerLawyer problem: An investigation of reasoners' underutilization of complementarity. Memory \& Cognition, 29, 79-91.

Bar-Hillel, M. (1980). The base-rate fallacy in probability judgments. Acta Psychologica, $44,211-233$.

Bar-Hillel, M. (1983). The base rate fallacy controversy. In R. W. Scholz (Ed.). Decision making under uncertainty: Cognitive decision research, social interaction, development and epistemology. (pp. 39-61). Amsterdam: Elsevier Science.

Bar-Hillel, M. (1990). Back to base rates. In R. M. Hogarth (Ed.). Insights into decision making: A tribute to Hillel J. Einhorn. (pp. 200-216). Chicago: The University of Chicago Press.

Baron, J. (1994). Thinking and deciding (2nd ed.). New York: Cambridge University Press.

Bayes, T. (1958). Essay towards solving a problem in the doctrine of chances. Biometrika, 45, 293-315. (Original work published 1763)

Beckett, E. N., \& Park, B. (1995). Use of category versus individuating information: Making base rates salient. Personality and Social Psychology Bulletin, 21, 21-31.

Bernoulli, J. (1987). Ars Conjectandi [Art of Conjecturing]. (N. Meusnier, Trans.). Rouen, France: IREM. (Original work published 1713)

Birnbaum, M. H. (2001). Introduction to behavioral research on the internet. Upper Saddle River, NJ: Prentice Hall. 
Birnbaum, M. H., \& Mellers, B. A. (1983). Bayesian inference: Combining base rates with opinions of sources who vary in credibility. Journal of Personality and Social Psychology, 45, 792-804.

Book, S. A. (1977). Statistics: Basic techniques for solving applied problems. New York: McGraw-Hill.

Borgida, E., \& Brekke, N. (1981). The base-rate fallacy in attribution and prediction. In J. H. Harvey, I. W \& K. R. F (Eds.), New directions in attribution research. (Vol. 3, pp. 63-95). Hillsdale, NJ: Erlbaum.

Budescu, D. V., \& Wallsten, T. S. (1985). Consistency in interpretation of probabilistic phrases. Organizational Behavior and Human Decision Processes, 36, 391-405.

Budnick, F. S. (1988). Applied mathematics for business, economics and the social sciences (3rd ed.). London: McGraw-Hill.

Byrne, E. F. (1968). Probability and Opinion. The Hague, The Netherlands: Martinus Nijhoff.

Carroll, J. S., \& Siegler, R. S. (1977). Strategies for the use of base-rate information. Organizational Behavior and Human Performance, 19, 392-402.

Christensen-Szalanski, J. J., \& Beach, L. R. (1982). Experience and the base-rate fallacy. Organizational Behavior and Human Decision Processes, 29, 270-278.

Chalker, S., \& Weiner, E. (1994). The Oxford dictionary of English grammar. London: Oxford University Press.

Cohen, J. (1960). Chance, skill and luck. The psychology of guessing and gambling. Harmondsworth, United Kingdom: Penguin Books.

Cohen, J. (1972). Psychological probability: The art of doubt. London: Allen and Unwin.

Cohen, L. J. (1979). On the psychology of prediction: Whose is the fallacy? Cognition, 7 , 385-407.

Colman, A. M. (2001). Set theory. In Dictionary of psychology (pp. 671). Oxford: Oxford University Press.

Cosmides, L., \& Tooby, J. (1996). Are humans good intuitive statisticians after all? Rethinking some conclusions from the literature on judgment under uncertainty. Cognition, 58, 1-73.

Daston, L. (1988). Classical probability in the enlightenment. Chichester, United Kingdom: Princeton University Press.

De Finetti, B. (1964). Foresight: Its logical laws, its subjective sources. In H. E. Kyburg $\&$ H. E. Smokler (Eds.), Studies in subjective probability. (pp. 93-158). New York: John Wiley \& Sons. (Original work published 1937)

Dougherty, M. R. P., Gettys, C. F., \& Ogden, E. E. (1999). MINERVA-DM: A memory processes model for judgments of likelihood. Psychological Review, 106, 180-209.

Eddy, D. M. (1982). Probabilistic reasoning in clinical medicine: Problems and opportunities. In D. Kahneman, P. Slovic \& A. Tversky (Eds.), Judgment under uncertainty: Heuristic and biases. (pp. 249-267). Cambridge, UK: Cambridge University Press.

Edwards, W. (1954). The theory of decision making. Psychological Bulletin, 51, 380-417. 
Edwards, W. (1961). Behavioral decision theory. Annual Review of Psychology, 12, 473 498.

Edwards, W. (1962). Dynamic decision theory and probabilistic information

processing. Human Factors, 4, 59-73.

Edwards, W. (1968). Conservatism in Human Information Processing. In B. Kleinmuntz (Ed.). Formal Representation of Human Judgment. (pp. 17-52). New York: Wiley.

Edwards, W., Lindman, H., \& Savage, L. J. (1963). Bayesian statistical inference for psychological research. Psychological Review, 70, 193-242.

Edwards, W., Phillips, L. D., Hays, W. L., \& Goodman, B. C. (1968). Probabilistic information processing systems: Design and evaluation. IEEE Transactions on Systems Science and Cybernetics, SSC-4, 248-265.

Einhorn, H. J., \& Hogarth, R. M. (1981). Behavioral decision theory: Processes of judgment and choice. Annual Review of Psychology, 32, 53-88.

Ellis, R. L. (1849). On the foundations of the theory of probabilities. Transactions of the Cambridge Philosophical Society, 8, 1-6.

Ericsson, K. A., \& Simon, H. A. (1993). Protocol analysis: Verbal reports as data (Rev. ed.). Cambridge, MA: MIT Press.

Evans, J. S. B. T. (1998). Matching bias in conditional reasoning: Do we understand it after 25 years? Thinking $\mathcal{B}$ Reasoning, 4, 45-82.

Evans, J. S. B. T., Handley, S. J., Perham, N., Over, D. E., \& Thompson, V. A. (2000). Frequency versus probability formats in statistical word problems. Cognition, 77, 197213.

Fiedler, K. (2000). Beware of samples! A cognitive-ecological sampling approach to judgment biases. Psychological Review, 107, 659-676.

Fienberg, S. E. (1992). A brief history of statistics in three and one-half chapters: A review essay. Statistical Science, 7, 208-225.

Fischhoff, B., \& Bar-Hillel, M. (1984). Diagnosticity and the base-rate effect. Memory $\mathbb{E}$ Cognition, 12, 402-410.

Fischhoff, B., \& Lichtenstein, S. (1978). Don't attribute this to Reverend Bayes. Psychological Bulletin, 85, 239-243.

Fischhoff, B., Slovic, P., \& Lichtenstein, S. (1979). Subjective sensitivity analysis. Organizational Behavior and Human Decision Processes, 23, 339-359.

Fox, J. (1994). On the necessity of probability: Reasons to believe and grounds for doubts. In G. Wright \& P. Ayton (Eds.), Subjective probability. (pp. 75-104). Oxford, United Kingdom: John Wiley \& Sons.

Gigerenzer, G. (1996). On narrow norms and vague heuristics: A reply to Kahneman and Tversky. Psychological Review, 103, 592-596.

Gigerenzer, G. (1997). Ecological intelligence: An adaption for frequencies. Psychologische Beiträge, 39, 107-125.

Gigerenzer, G., Hell, W., \& Blank, H. (1988). Presentation and content: The use of base rates as a continuous variable. Journal of Experimental Psychology: Human Perception and Performance, 14, 513-525. 
Gigerenzer, G., \& Hoffrage, U. (1995). How to improve Bayesian reasoning without instruction: Frequency formats. Psychological Review, 102, 684-704.

Gigerenzer, G., \& Hoffrage, U. (1999). Overcoming difficulties in Bayesian reasoning: A reply to Lewis and Keren (1999) and Mellers and McGraw (1999). Psychological Review, 106, 425-430.

Gigerenzer, G., \& Murray, D. J. (1987). Cognition as intuitive statistics. Hillsdale, NJ, England: Lawrence Erlbaum Associates.

Gigerenzer, G., Swijtink, Z. G., Porter, T. M., Daston, L., Beatty, J., \& Krüger, L. (1989). The empire of chance: How probability changed science and everyday life. Cambridge, United Kingdom: Cambridge University Press.

Gillies, D. A. (2000). Philosophical theories of probability. London: Routledge.

Ginossar, Z., \& Trope, Y. (1980). The effects of base rates and individuating information on judgments about another person. Journal of Experimental Social Psychology, 16, 228-242.

Ginossar, Z., \& Trope, Y. (1987). Problem solving in judgment under uncertainty. Journal of Personality and Social Psychology, 52, 464-473.

Girotto, V., \& Gonzalez, M. (2001). Solving probabilistic and statistical problems: A matter of information structure and question form. Cognition, 78, 247-276.

Girotto, V., \& Gonzalez, M. (2002). Chances and frequencies in probabilistic reasoning: Rejoinder to Hoffrage, Gigerenzer, Krauss and Martignon. Cognition, 84, 353-359.

Godfroy-Génin, A.-S. (2000). Pascal: La géométrie du hasard [Pascal: The geometry of hazard]. Mathématiques et Sciences Humaines, 150, 7-39.

Goldstein, W. M., \& Hogarth, R. M. (1997). Judgment and decision research: Some historical context. In W. M. Goldstein \& R. M. Hogarth (Eds.), Research on judgment and decision making: Currents, connections, and controversies. Cambridge: Cambridge University Press.

Griffin, D., \& Buehler, R. (1999). Frequency, probability, and prediction: Easy solutions to cognitive illusions? Cognitive Psychology, 38, 48-78.

Gröner, R., Gröner, M., \& Bischof, W. F. (1983). The role of heuristics in models of decision. In R. W. Scholz (Ed.). Decision making under uncertainty: Cognitive decision research, social interaction, development and epistemology. (pp. 87-108). Amsterdam: Elsevier Science.

Hacking, I. (1975). The emergence of probability. Cambridge, United Kingdom: Cambridge University Press.

Hamm, R. M. (1993). Explanations for common responses to the Blue/Green Cab probabilistic inference word problem. Psychological Reports, 72, 219-242.

Hamm, R. M. (1994). Underweighting of base-rate information reflects important difficulties people have with probabilistic inference. Retrieved June 9, 2003, from http://psycprints.ecs.soton.ac.uk/archive/00000362/02/psyc.94.5.03.baserate.7.hamm

Hamm, R. M. (1998). Characterizing individual strategies illuminates nonoptimal behavior. Retrieved June 9, 2003, from http://psycprints.ecs.soton.ac.uk/archive/00000598/02/psyc.98.9.49.socialbias.2.hamm 
Hammerton, M. (1973). A case of radical probability estimation. Journal of Experimental Psychology, 101, 252-254.

Hammond, K. R. (2000). Coherence and correspondence: Theories in judgment and decision making. In T. Connolly, H. R. Arkes \& K. R. Hammond (Eds.), Judgment and decision making: An interdisciplinary reader. Cambridge, United Kingdom: Cambridge University Press. (Original work published 1996)

Harries, C., \& Harvey, N. (2000). Are absolute frequencies, relative frequencies, or both effective in reducing cognitive biases? Journal of Behavioral Decision Making, 13, 431 444.

Hertwig, R., \& Todd, P. M. (2000). Biases to the left, fallacies to the right: Stuck in the middle with null hypothesis significance testing. Retrieved June 9, 2003, from http://psycprints.ecs.soton.ac.uk/archive/00000028/02/psyc.00.11.028.socialbias.20.hertwig

Hewstone, M. (1989). Causal attribution: From cognitive processes to collective beliefs. Oxford, UK: Blackwell.

Hinsz, V. B., Tindale, R. S., Nagao, D. H., Davis, J. H., \& Robertson, B. A. (1988). The influence of the accuracy of the individuating information on the use of base rate information in probability judgment. Journal of Experimental Social Psychology, 24, $127-145$.

Hoffrage, U., Gigerenzer, G., Krauss, S., \& Martignon, L. (2002). Representation facilitates reasoning: What natural frequencies are and what they are not. Cognition, $84,343-352$.

Hogarth, R. M. (1975). Cognitive processes and the assessment of subjective probability distributions. Journal of the American Statistical Association, 70, 271-289.

Howell, D. C. (1992). Statistical methods for psychology (3rd ed.). Boston, MS: PWSKent.

Howell, W. C., \& Burnett, S. A. (1978). Uncertainty measurement: A cognitive taxonomy. Organizational Behavior and Human Decision Processes, 22, 45-68.

Howson, C., \& Urbach, P. (1993). Scientific reasoning: The Bayesian approach (2nd ed.). Chicago, IL: Open Court.

Hume. (2000). A treatise of human nature: Being an attempt to introduce the experimental method of reasoning into moral subjects. Oxford, United Kingdom: Oxford University Press. (Original work published 1739)

Johnson-Laird, P. N. (1983). Mental Models: Towards a cognitive science of language, inference, and consciousness. Cambridge: Cambridge University Press.

Johnson-Laird, P. N., \& Byrne, R. M. J. (1993). Précis of deduction. Behavioral and Brain Sciences, 16, 323-380.

Johnson-Laird, P. N., Legrenzi, P., Girotto, V., Sonino-Legrenzi, M., \& Caverni, J. P. (1999). Naive probability: A mental model theory of extensional reasoning. Psychological Review, 106, 62-88.

Kahneman, D., Slovic, P., \& Tversky, A. (Eds.). (1982). Judgment under uncertainty: Heuristic and biases. Cambridge, UK: Cambridge University Press.

Kahneman, D., \& Tversky, A. (1973). On the psychology of prediction. Psychological Review, 80, 237-251. 
Kahneman, D., \& Tversky, A. (1982a). Intuitive prediction: Biases and corrective procedures. In D. Kahneman, P. Slovic \& A. Tversky (Eds.), Judgement under uncertainty: Heuristics and biases. (pp. 414-421). Cambridge, UK: Cambridge University Press. (Original work published 1979)

Kahneman, D., \& Tversky, A. (1982b). On the study of statistical intuitions. In D. Kahneman, P. Slovic \& A. Tversky (Eds.), Judgment under uncertainty: Heuristics and biases. (pp. 493-508). Cambridge, UK: Cambridge University Press. (Original work published 1982)

Kahneman, D., \& Tversky, A. (1982c). Subjective probability: A judgment by representativeness. In D. Kahneman, P. Slovic \& A. Tversky (Eds.), Judgement under uncertainty: Heuristics and biases. (pp. 32-47). Cambridge, UK: Cambridge University Press. (Original work published 1972)

Kahneman, D., \& Tversky, A. (1982d). Variants of uncertainty. In D. Kahneman, P. Slovic \& A. Tversky (Eds.), Judgement under uncertainty: Heuristics and biases. (pp. 509-520). Cambridge, UK: Cambridge University Press. (Original work published 1982)

Kahneman, D., \& Tversky, A. (1996). On the reality of cognitive illusions. Psychological Review, 103, 582-591.

Kendall, M. G. (1956). The beginnings of a probability calculus. Biometrika, 43, 1-14.

Keren, G., \& Teigen, K. H. (2001). The probability-outcome correspondence principle: A dispositional view of the interpretation of probability statements. Memory $\mathbb{E}$ Cognition, 29, 1010-1021.

Koehler, J. J. (1996). The Base rate fallacy reconsidered: Normative, descriptive and methodological challenges. Behavioral and Brain Sciences, 19, 1-17.

Koehler, J. J. (2001). When are people persuaded by DNA match statistics? Law and Human Behavior, 25, 493-513.

Krosnick, J. A., Li, F., \& Lehman, D. R. (1990). Conversational conventions, order of information acquisition, and the effect of base rates and individuating information on social judgments. Journal of Personality and Social Psychology, 39, $1140-1152$.

Krueger, J. (1998). The bet on bias: A foregone conclusion? Retrieved June 9, 2003, from http://psycprints.ecs.soton.ac.uk/archive/00000595/02/psyc.98.9.46.socialbias.1.krueger

Krueger, J., \& Rothbart, M. (1988). Use of categorical and individuating information in making inferences about personality. Journal of Personality and Social Psychology, 55, 187-195.

Kyburg, H. E., \& Smokler, H. E. (Eds.). (1964). Studies in subjective probability. New York: John Wiley \& Sons.

Lagnado, D. A., \& Shanks, D. R. (2002). Probability judgment in hierarchical learning: a conflict between predictiveness and coherence. Cognition, 83, 81-112.

Laplace, P. S. (1951). A philosophical essay on probabilities. (F. W. Truscott \& F. L. Emory, Trans.). New York: Dover Publications. (Original work published 1814)

Laplace, P. S. (1986). Memoir on the probability of the causes of events. Statistical Science, 1, 364-378. (Original work published 1774) 
Liu, A. Y. (1975). Specific information effect in probability estimation. Perceptual and Motor Skills, 41, 475-478.

Locksley, A., Borgida, E., Brekke, N., \& Hepburn, C. (1980). Sex stereotypes and social judgment. Journal of Personality and Social Psychology, 39, 821-831.

Locksley, A., Hepburn, C., \& Ortiz, V. (1982). Social stereotypes and judgments of individuals: An instance of the base-rate fallacy. Journal of Experimental Social Psychology, 18, 23-42.

Lopes, L. L. (1981). Performing competently. Behavioral and Brain Sciences, 4, 343-344.

Lynch, J. G., \& Ofir, C. (1989). Effects of cue consistency and value on base-rate utilization. Journal of Personality and Social Psychology, 56, 170-181.

Lyon, D., \& Slovic, P. (1976). Dominance of accuracy information and neglect of base rates in probability estimation. Acta Psychologica, 40, 286-298.

Macchi, L. (1995). Pragmatic aspects of the base-rate fallacy. The Quaterly Journal of Experimental Psychology, 48A, 188-207.

Macchi, L. (2000). Partitive formulation of information in probabilistic problems: Beyond heuristics and frequency format explanations. Organizational Behavior and Human Decision Processes, 82, 217-236.

Macchi, L., \& Mosconi, G. (1998). Computational features vs frequentist phrasing in the base-rate fallacy. Swiss Journal of Psychology, 57, 79-85.

Macchi, L., Osherson, D., \& Krantz, D. H. (1999). A note on superadditive probability judgment. Psychological Review, 106, 210-214.

Manktelow, K. (1999). Reasoning and thinking. Hove, United Kingdom: Psychology Press.

Marr, D. (1982). Vision: A computational investigation into the human representation and processing of visual information. San Francisco: W. H. Freeman.

McCauley, C., \& Stitt, C. L. (1978). An individual and quantitative measure of stereotypes. Journal of Personality and Social Psychology, 36, 929-940.

Mellers, B. A., \& McGraw, A. P. (1999). How to improve Bayesian reasoning: Comment on Gigerenzer and Hoffrage (1995). Psychological Review, 106, 417 424.

Mill, J. S. (1973). A system of logic ratiocinative and inductive. London: Routledge \& Kegan Paul. (Original work published 1843)

Mosteller, F., Rourke, R. E. K., \& Thomas, G. B. (Eds.). (1970). Probability: A first course (2nd ed.). London: Addison-Wesley.

Newell, A., \& Simon, H. A. (1972). Human problem solving. Englewood Cliffs, NJ: Prentice Hall.

Nisbett, R. E., \& Borgida, E. (1975). Attribution and the psychology of prediction. Journal of Personality and Social Psychology, 32, 932-943.

Nisbett, R. E., Borgida, E., Crandall, R., \& Reed, H. (1982). Popular induction: Information is not necessarily informative. In D. Kahneman, P. Slovic \& A. Tversky (Eds.), Judgement under uncertainty: Heuristics and biases. (pp. 101-116). Cambridge, United Kingdom: Cambridge University Press. (Original work published 1976) 
Oaksford, M., \& Chater, N. (1998). Rationality in an uncertain world. Hove, United Kingdom: Psychology Press.

Oaksford, M., \& Chater, N. (2001). The probabilistic approach to human reasoning. Trends in Cognitive Sciences, 5, 349-357.

Ofir, C., \& Lynch, J. G. (1984). Context effects on judgment under uncertainty. The Journal of Consumer Research, 11, 668-679.

O'Sullivan, N. (1995). Aristotle on dramatic probability. Classical Journal, 91, 47-63.

Pascal, B. (1963). La règle des partis. [The rule of divisions]. In B. Pascal, Oeuvres Complètes. Paris: Editions du Seuil. (Original work published 1654)

Peterson, C. R., Schneider, R. J., \& Miller, A. J. (1965). Sample size and the revision of subjective probability. Journal of Experimental Psychology, 69, 522-527.

Phillips, L. D. (1970). The "true probability" problem. Acta Psychologica, 34, 254-264.

Phillips, L. D., \& Edwards, W. (1966). Conservatism in a simple probability model inference task. Journal of Experimental Psychology, 72, 346-354.

Phillips, L. D., Hays, W. L., \& Edwards, W. (1966). Conservatism in complex probabilistic inference. IEEE Transactions on Human Factors in Electronics, 7, 718.

Politzer, G., \& Macchi, L. (2000, August). The representation of the task in the lawyerengineer problem. Poster presented at the Thinking 2000 Conference, Durham, United Kingdom.

Pollard, P., \& Evans, J. S. B. T. (1983). The role of 'representativeness' in statistical inference: A critical appraisal. In J. S. B. Evans (Ed.). Thinking and reasoning: Psychological approaches. (pp. 107-134). London: Routledge \& Kegan Paul.

Porter, T. M. (1986). The rise of statistical thinking: 1820-1900. Guildford, United Kingdom: Princeton University Press.

Ramsey, F. P. (1964). Truth and Probability. In H. E. Kyburg \& H. E. Smokler (Eds.), Studies in subjective probability. (pp. 61-92). New York: John Wiley \& Sons. (Original work published 1931)

Rankin, B. (1966). The history of probability and the changing concept of the individual. Journal of the History of Ideas, 27, 483-504.

Rapoport, A., \& Wallsten, T. S. (1972). Individual decision behavior. Annual Review of Psychology, 23, 131-176.

Rasinski, K. A., Crocker, J., \& Hastie, R. (1985). Another look at sex stereotypes and social judgments: An analysis of the social perceiver's use of subjective probabilities. Journal of Personality and Social Psychology, 49, 317-326.

Reichenbach, H. (1949). The logical foundations of the concept of probability. In H. Feigl \& W. Sellars (Eds.), Readings in philosophical analysis. New York: Appleton Century Crofts. (Original work published 1932)

Rottenstreich, Y., \& Tversky, A. (1997). Unpacking, repacking, and anchoring: Advances in support theory. Psychological Review, 104, 406-415.

Savage, L. J. (1972). The foundations of statistics. New York: Dover Publications. (Original work published 1954) 
Schmitz, T. A. (2000). Plausibility in the Greek orators. American Journal of Philology, $121,47-77$.

Schwarz, N., Strack, F., Hilton, D., \& Naderer, G. (1991). Base rates, representativeness, and the logic of conversation: The contextual relevance of 'irrelevant' information. Social Cognition, 9, 67-83.

Selling, T., \& Shank, J. (1989). Linear versus process tracing approaches to judgment modelling: A new perspective on cue importance. Accounting, Organizations and Society, 14, 65-77.

Shafer, G. (1990). The unity and diversity of probability. Statistical Science, 5, 435-444.

Shafer, G. (1996). The significance of Jacob Bernoulli's Ars Conjectandi for the philosophy of probability today. Journal of Econometrics, 75, 15-32.

Sherman, S. J., \& Corty, E. (1984). Cognitive heuristics. In R. S. Wyer \& T. K. Srull (Eds.), Handbook of social cognition. (Vol. 1, pp. 189-286). Hillsdale, NJ: Erlbaum.

Sheynin, O. B. (1974). On the prehistory of the theory of probability. Archive for History of Exact Sciences, 12, 97-141.

Shye, S., Elizur, D., \& Hoffman, M. (1994). Applied social research methods series: Vol. 35. Introduction to facet theory: Content design and intrinsic data analysis in behavioral research. Thousand Oaks, CA: Sage Publications.

Skov, R. B., \& Sherman, S. J. (1986). Information-gathering processes: Diagnosticity, hypothesis-confirmatory strategies, and perceived hypothesis confirmation. Journal of Experimental Social Psychology, 22, 93-121.

Sloman, S. A., Over, D., \& Slovak, L. (2003). Frequency Illusions and Other Fallacies. Organizational Behavior and Human Decision Processes, 91, 296-309.

Slovic, P., Fischhoff, B., \& Lichtenstein, S. (1977). Behavioral decision theory. Annual Review of Psychology, 28, 1-39.

Slovic, P., \& Lichtenstein, S. (1971). Comparison of Bayesian and regression approaches to the study of information processing in judgment. Organizational Behavior and Human Performance, 6, 649-744.

Slowiaczek, L. M., Klayman, J., Sherman, S. J., \& Skov, R. B. (1992). Information selection and use in hypothesis testing: What is a good question, and what is a good answer? Memory Eु Cognition, 20, 392-405.

Stanovich, K. E., \& West, R. F. (1998). Who uses base rates and $\mathrm{P}(\mathrm{D} / \sim \mathrm{H})$ ? An analysis of individual differences. Memory and Cognition, 26, 161-179.

Tabachnick, B. G., \& Fidell, L. S. (1996). Using multivariate statistics (3rd ed.). New York: Harper Collins.

Tabachnick, B. G., \& Fidell, L. S. (2001). Computer-assisted research design and analysis. Needham Heights, MA: Allyn \& Bacon.

Teigen, K. H. (1974). Overestimation of subjective probabilities. Scandinavian Journal of Psychology, 15, 56-62.

Teigen, K. H. (1988). The language of uncertainty. Acta Psychologica, 68, 27-38.

Teigen, K. H. (1994). Variants of subjective probabilities: Concepts, norms, and biases. In G. Wright \& P. Ayton (Eds.), Subjective probability. (pp. 211-238). Oxford, United Kingdom: John Wiley \& Sons. 
Teigen, K. H., \& Brun, W. (1995). Yes, but it is uncertain: Direction and communicative intention of verbal probabilistic terms. Acta Psychologica, 88, 233-258.

Teigen, K. H., \& Brun, W. (2003). Verbal probabilities: A question of frame? Journal of Behavioral Decision Making, 16, 53-72.

Todhunter, I. (1949). A history of the mathematical theory of probability from the time of Pascal to that of Laplace. New York: Chelsea. (Original work published 1865)

Tversky, A., \& Kahneman, D. (1980). Causal schemas in judgments under uncertainty. In M. Fishbein (Ed.). Progress in social psychology. (pp. 49-72). Hillsdale, N. J.: Erlbaum.

Tversky, A., \& Kahneman, D. (1982a). Evidential impact of base rates. In D. Kahneman, P. Slovic \& A. Tversky (Eds.), Judgment under uncertainty: Heuristic and biases. (pp. 153-160). Cambridge, UK: Cambridge University Press.

Tversky, A., \& Kahneman, D. (1982b). Judgment under uncertainty: Heuristics and biases. In D. Kahneman, P. Slovic \& A. Tversky (Eds.), Judgement under uncertainty: Heuristics and biases. (pp. 3-20). Cambridge, UK: Cambridge University Press. (Original work published 1974)

Tversky, A., \& Kahneman, D. (1982c). Judgments of and by representativeness. In D. Kahneman, P. Slovic \& A. Tversky (Eds.), Judgment under uncertainty: Heuristic and biases. (pp. 84-98). Cambridge, UK: Cambridge University Press.

Tversky, A., \& Kahneman, D. (1983). Extensional versus intuitive reasoning: The conjunction fallacy in probability judgment. Psychological Review, 90, 293-315.

Tversky, A., \& Koehler, D. J. (1994). Support theory: A nonextensional representation of subjective probability. Psychological Review, 101, 547-567.

Vail, S. (1954). Alternative calculi of subjective probabilities. In R. M. Thrall, C. H. Coombs \& R. L. Davis (Eds.), Decision processes. (pp. 87-98). Oxford, United Kingdom: John Wiley \& Sons.

Vaught, R. L. (1995). Set theory: An introduction (2nd ed.). Boston: Birkhäuser.

Venn, J. (1962). The logic of chance. New York: Chelsea. (Original work published 1866)

Von Mises, R. (1957). Probability, statistics and truth. London: Allen \& Unwin. (Original work published 1928)

Von Plato, J. (1994). Creating modern probability: Its mathematics, physics and philosophy in historical perspective. Cambridge: Cambridge University Press.

Wallsten, T. S. (1980). Processes and models to describe choice and inference behavior. In T. S. Wallsten (Ed.). Cognitive processes in choice and decision behavior. (pp. 215-237). Hillsdale, NJ: Lawrence Erlbaum Associates.

Wallsten, T. S. (1983). The theoretical status of judgmental heuristics. In R. W. Scholz (Ed.). Advances in psychology: Vol. 16. Decision making under uncertainty: Cognitive decision research, social interaction, development and epistemology. (pp. 21-37). Amsterdam: Elsevier Science.

Wallsten, T. S., Budescu, D. V., Rapoport, A., Zwick, R., \& Forsyth, B. (1986). Measuring the vague meanings of probability terms. Journal of Experimental Psychology: General, 115, 348-365. 
Wason, P. C. (1966). Reasoning. In F. B. M (Ed.). New Horizons in Psychology. (pp. 135151). Harmondsworth, UK: Penguin.

Wason, P. C., \& Evans, J. S. B. T. (1975). Dual processes in reasoning? Cognition, 3, $141-154$.

Wells, G. L., \& Harvey, J. H. (1978). Naive attributors' attributions and predictions: What is informative and when is an effect an effect? Journal of Personality and Social Psychology, 36, 483-490.

Wilkinson, S. (2003). Focus groups. In J. A. Smith (Ed.). Qualitative psychology: A practical guide to research methods. London: Sage publications.

Wolfe, C. R. (1995). Information seeking on Bayesian conditional probability problems: A fuzzy-trace theory account. Journal of Behavioral Decision Making, 8, 85-108.

Zukier, H., \& Pepitone, A. (1984). Social roles and strategies in prediction: Some determinants of the use of base-rate information. Journal of Personality and Social Psychology, 47, 349-360. 
The odds form of Bayes' theorem reads as follows,

$$
\frac{p(H \mid D)}{p(\sim H \mid D)}=\frac{p(H)}{p(\sim H)} \cdot \frac{p(D \mid H)}{p(D \mid \sim H)}
$$

When $p(H)=c$, Equation (A.1) becomes,

$$
\frac{p(H \mid D)_{\mathrm{High}}}{p(\sim H \mid D)_{\mathrm{High}}}=\frac{c}{1-c} \cdot \frac{p(D \mid H)}{p(D \mid \sim H)} .
$$

Similarly, when $p(H)=1-c$, Equation (A.1) becomes,

$$
\frac{p(H \mid D)_{\text {Low }}}{p(\sim H \mid D)_{\text {Low }}}=\frac{1-c}{c} \cdot \frac{p(D \mid H)}{p(D \mid \sim H)}
$$

Assuming that $p(D \mid H)$ and $p(D \mid \sim H)$ are independent of the value of $p(H)$, the normative relation $p(H \mid D)_{\text {High }}$ and $p(H \mid D)_{\text {Low }}$ is then derived from the ratio of Equations (A.2) and (A.3),

$$
\frac{\frac{p(H \mid D)_{\text {High }}}{p(\sim H \mid D)_{\text {High }}}}{\frac{p(H \mid D)_{\text {Low }}}{p(\sim H \mid D)_{\text {Low }}}}=\frac{c}{\frac{1-c}{c}} \cdot \frac{\frac{p(D \mid H)}{p(D \mid \sim H)}}{\frac{p(D \mid H)}{p(D \mid \sim H)}}=\left(\frac{c}{1-c}\right)^{2} .
$$

Let $k^{2}$ refer to $\left(\frac{c}{1-c}\right)^{2}$. Equation (A.4) becomes,

$$
\begin{aligned}
& \frac{\frac{p(H \mid D)_{\text {High }}}{p(\sim H \mid D)_{\text {High }}}}{\frac{p(H \mid D)_{\text {Low }}}{p(\sim H \mid D)_{\text {Low }}}}=k^{2} \Leftrightarrow \frac{p(H \mid D)_{\text {High }}}{p(\sim H \mid D)_{\text {High }}}=k^{2} \cdot \frac{p(H \mid D)_{\text {Low }}}{p(\sim H \mid D)_{\text {Low }}} \\
& \Leftrightarrow p(H \mid D)_{\mathrm{High}} \cdot p(\sim H \mid D)_{\mathrm{Low}}=k^{2} \cdot p(H \mid D)_{\mathrm{Low}} \cdot p(\sim H \mid D)_{\mathrm{High}} .
\end{aligned}
$$

Since $p(H \mid D)=1-p(\sim H \mid D)$, it follows that, 


$$
p(H \mid D)_{\text {High }} \cdot\left[1-p(H \mid D)_{\text {Low }}\right]=k^{2} \cdot p(H \mid D)_{\text {Low }} \cdot\left[1-p(H \mid D)_{\text {High }}\right]
$$

that is,

$$
\begin{aligned}
p(H \mid D)_{\mathrm{High}}-p(H \mid D)_{\mathrm{High}} \cdot p(H \mid D)_{\mathrm{Low}} \\
=k^{2} \cdot p(H \mid D)_{\mathrm{Low}}-k^{2} \cdot p(H \mid D)_{\mathrm{Low}} \cdot p(H \mid D)_{\mathrm{High}} .
\end{aligned}
$$

The of $p(H \mid D)_{\text {Low }}$ as a function of $p(H \mid D)_{\text {High }}$ can be inferred by rearranging Equation (A.7),

$$
\begin{aligned}
p(H \mid D)_{\text {High }} & \\
& =k^{2} \cdot p(H \mid D)_{\mathrm{Low}}-k^{2} \cdot p(H \mid D)_{\mathrm{Low}} \cdot p(H \mid D)_{\mathrm{High}}+p(H \mid D)_{\mathrm{High}} \cdot p(H \mid D)_{\mathrm{Low}}(\mathrm{A} . \\
& =p(H \mid D)_{\mathrm{Low}}\left[k^{2}-k^{2} \cdot p(H \mid D)_{\mathrm{High}}+p(H \mid D)_{\mathrm{High}}\right]
\end{aligned}
$$

that is,

$$
p(H \mid D)_{\text {Low }}=\frac{p(H \mid D)_{\mathrm{High}}}{k^{2}+p(H \mid D)_{\mathrm{High}}\left(1-k^{2}\right)} .
$$

Similarly, the value of $p(H \mid D)_{\text {High }}$ as a function of $p(H \mid D)_{\text {Low }}$ can be inferred from Equation (A.7) as,

$$
p(H \mid D)_{\text {High }}\left[1-p(H \mid D)_{\text {Low }}+k^{2} \cdot p(H \mid D)_{\text {Low }}\right]=k^{2} \cdot p(H \mid D)_{\text {Low }},
$$

that is,

$$
p(H \mid D)_{\text {High }}=\frac{k^{2} \cdot p(H \mid D)_{\text {Low }}}{1+p(H \mid D)_{\text {Low }}\left(k^{2}-1\right)} .
$$

sk 


\section{Probability interpretation task}

Please, state how you would define the meaning of the following statement:

"There is a $30 \%$ chance that an applicant will get a position given that he failed the psychometric tests."

\section{Table B1}

Transcripts for each of the different coding categories $(\mathrm{N}=80)$.

\begin{tabular}{|c|c|}
\hline Participant & Statements \\
\hline \multicolumn{2}{|r|}{ Natural frequency interpretations } \\
\hline 1 & $\begin{array}{l}\text { Irrespective of those that passed the psychometric tests, just under one third of the } \\
\text { applicants that failed the psychometric tests will still be employed by the company. }\end{array}$ \\
\hline 5 & One third of the people that fail the psychometric test will get a position. \\
\hline 9 & Out of every 100 people who fail the tests, 30 will get the position. \\
\hline 10 & That 30 out of 100 people will get a job even if he fails the psychometric tests. \\
\hline 19 & Of the people who failed the test 3 out of 10 would get the job. \\
\hline 20 & $\begin{array}{l}\text { Of those candidates who failed the psychometric test, three out of ten are still likely to } \\
\text { get a position. }\end{array}$ \\
\hline 22 & $\begin{array}{l}3 \text { in ten people who fail a psychometric test will get th job they applied for. But } 7 \text { in ten } \\
\text { who fail a psychometric test will not get the job they applied for. Therefore of you fail a } \\
\text { psychometric test you are less likely to get the job applied for. }\end{array}$ \\
\hline 23 & $\begin{array}{l}7 \text { out of every } 10 \text { applicants will fail to get a position regardless of whether they pass the } \\
\text { psychometric tests or not. }\end{array}$ \\
\hline 27 & Of every ten applicants who fail the psychometric tests, 3 will nevertheless get positions. \\
\hline 36 & $\begin{array}{l}\text { Um, as wrong. ( } 4 \text { I out of } 100 \text { people failed the psychometric test; } 6 \text { of them got the job } \\
\text { anyway. That's about 15\%). }\end{array}$ \\
\hline 37 & $\begin{array}{l}\text { Although the person had not passed the test helshe must have however accomplished } \\
\text { the interview. That means } 30 \text { out of } 100 \text { applicants who failed the test will get the } \\
\text { position. }\end{array}$ \\
\hline 39 & 3 out of 10 applicants who failed the test, will get the job regardless. \\
\hline 41 & 3 out of every 100 will get the job despite failing the test. \\
\hline 43 & 30 out of every 100 people who fail the psychometric tests get the position applied for. \\
\hline 45 & $\begin{array}{l}30 \text { out of every } 100 \text { people who fail the psychometric test will still gain a position } \\
\text { despite this. }\end{array}$ \\
\hline 52 & $\begin{array}{l}\text { Factors other than the results of the psychometric test results effect an applicant's } \\
\text { likelihood of being offered a position. Out of every } 100 \text { applicants who did not pass the } \\
\text { psychometric test, it is probable that } 30 \text { will be offered positions. }\end{array}$ \\
\hline 59 & $\begin{array}{l}\text { Statistically, out of } 10 \text { people who failed the psychometric tests, } 3 \text { will nevertheless get a } \\
\text { position. }\end{array}$ \\
\hline 62 & Nearly I in 3 get the job even though they fail the psychometric tests. \\
\hline 67 & 30 of every 100 people who have failed the psychometric test will be given a job. \\
\hline 69 & 3 out of every 10 applicants who fail the psychometric tests will get the position anyway. \\
\hline 70 & $\begin{array}{l}\text { Nearly I in } 3 \text { people who apply will get the position even if they have failed their } \\
\text { psychometric tests. }\end{array}$ \\
\hline 71 & $\begin{array}{l}\text { Out of the people that fail the psychometric test, } 30 \text { in } 100 \text { of them will still be offered } \\
\text { the position. }\end{array}$ \\
\hline 73 & $\begin{array}{l}\text { If an applicant fails the psychometric tests, he has a } 30 \% \text { chance of getting the position. } \\
\text { Or: Of every } 100 \text { people who fail the psychometric tests } 30 \text { will still get the position. }\end{array}$ \\
\hline
\end{tabular}




\begin{tabular}{|c|c|}
\hline Participant & Statements \\
\hline \multicolumn{2}{|r|}{ Relative frequency interpretations } \\
\hline 6 & $\begin{array}{l}\text { Of the number who fail the psychometric tests } 30 \text { per cent of that number will } \\
\text { nevertheless be offered a position. }\end{array}$ \\
\hline 12 & $\begin{array}{l}\text { There is a } 30 \% \text { chance that the individual will get a position even though they failed } \\
\text { the psychometric test. Therefore out of all the people who failed the psychometric } \\
\text { tests. } 30 \% \text { were still likely to get a position. [italics added] }\end{array}$ \\
\hline 24 & $\begin{array}{l}\text { Out of all the people who fail the psychometric tests, only } 30 \% \text { will have a chance in } \\
\text { getting the position. }\end{array}$ \\
\hline 42 & Of those people that failed a psychometric test, $30 \%$ will still be offered a position. \\
\hline 47 & $\begin{array}{l}\text { Out of the total number of applicants that failed the psychometric test, } 30 \% \text { will get } \\
\text { a position. }\end{array}$ \\
\hline 51 & $\begin{array}{l}30 \% \text { of the total number of applicants who failed the psychometric tests will still get } \\
\text { a position. }\end{array}$ \\
\hline 56 & Of those applicants who fail the psychometric tests, $30 \%$ still get a position. \\
\hline 57 & $\begin{array}{l}\text { From the } 100 \text { people that fail the psychometric test, } 30 \% \text { of them could get a } \\
\text { position. }\end{array}$ \\
\hline 64 & $\begin{array}{l}\text { Of the total number of applicants some pass the psychometric tests and may or may } \\
\text { not get the job. Some fail the tests. } 30 \% \text { of those that fail will get the job. }\end{array}$ \\
\hline 72 & $30 \%$ of people who fail the test will still get the position! \\
\hline 80 & $\begin{array}{l}\text { Only taking into account applicants who failed, } 30 \% \text { of them have a chance to get } \\
\text { the position. }\end{array}$ \\
\hline \multicolumn{2}{|r|}{ Other frequency interpretations } \\
\hline 65 & $\begin{array}{l}\text { The ratio of the number of applicants who failed the psychometric tests and got a } \\
\text { position to the total number of applicants who failed the psychometric tests is } 30 \text {. }\end{array}$ \\
\hline \multicolumn{2}{|r|}{ Natural chance interpretations } \\
\hline 2 & $\begin{array}{l}\text { If the applicant fails the test he/she still has (slightly under) a I in } 3 \text { chance of getting } \\
\text { a position. }\end{array}$ \\
\hline 3 & $\begin{array}{l}\text { If you fail to pas the psychometric test, you will have a chance of I in } 3 \text { to get a } \\
\text { position. }\end{array}$ \\
\hline 7 & $\begin{array}{l}\text { There is a } 3 \text { out of } 10 \text { chance that an applicant will receive a position regarding that } \\
\text { they failed the psychometric test. }\end{array}$ \\
\hline 18 & $\begin{array}{l}\text { If an applicant for a given position fails the psychometric test, he has only } 3 \text { chances } \\
\text { in ten to get the job. }\end{array}$ \\
\hline 40 & $\begin{array}{l}\text { There is a } 3 \text { out of } 10 \text { chances that you will get the position given you failed the } \\
\text { psychometric test. }\end{array}$ \\
\hline 48 & $\begin{array}{l}\text { Despite failing psychometric tests a candidate still has a I in } 3 \text { chance of being } \\
\text { offered a position. }\end{array}$ \\
\hline 49 & $\begin{array}{l}\text { That if the applicant failed the test, there would be } 30 / 100 \text { chances that he will get } \\
\text { the position. }\end{array}$ \\
\hline 54 & $\begin{array}{l}\text { If the applicant failed the psychometric test, he has a I in } 3 \text { chance of getting a } \\
\text { position (which is not true as there are only } 4 \text { positions left)! }\end{array}$ \\
\hline 61 & There is 3 chances out of 10 to get the job, if you have failed the psychometric test. \\
\hline 63 & $\begin{array}{l}\text { If you fail a psychometric test there is a three in ten chance that you will actually get } \\
\text { a position. }\end{array}$ \\
\hline 66 & $\begin{array}{l}\text { If someone fails the tests, they still have a } 30 \text { in } 100 \text { chance of attaining the position } \\
\text { anyway. }\end{array}$ \\
\hline 68 & $\begin{array}{l}\text { There are } 30 \text { chances out of } 100 \text { chances that an applicant will get a position given } \\
\text { that he failed the psychometric test }\end{array}$ \\
\hline 77 & Having failed the psychometric test, he has a 30 in 100 chance of getting the job. \\
\hline
\end{tabular}




\begin{tabular}{|c|c|}
\hline Participant & Statements \\
\hline \multicolumn{2}{|r|}{ Relative chance interpretations } \\
\hline 4 & $\begin{array}{l}\text { If a person has failed the tests, there is still a } 30 \% \text { chance that they will get a position } \\
\text { anyway }\end{array}$ \\
\hline 8 & $\begin{array}{l}\text { If though an applicant fails the psychometric test, there is still a } 30 \% \text { chance of the } \\
\text { applicant getting the position. }\end{array}$ \\
\hline 13 & $\begin{array}{l}\text { All the applicants who failed the psychometric tests still have } 30 \% \text { chance to get a } \\
\text { position given. }\end{array}$ \\
\hline 17 & If you failed the tests then you still have a $30 \%$ chance of getting a position. \\
\hline 26 & $\begin{array}{l}\text { Even if the applicant failed the psychometric test there is still a } 30 \% \text { chance they will } \\
\text { get the job. }\end{array}$ \\
\hline 29 & $\begin{array}{l}\text { This means that if an applicant failed a psychometric test, they would still have a } 30 \% \\
\text { chance of still actually get a job. }\end{array}$ \\
\hline 31 & Even if you fail the psychometric test, you have a $30 \%$ chance of getting the position. \\
\hline 32 & $\begin{array}{l}\text { If you fail the psychometric test, you have only a } 30 \% \text { (less that a I in 3) chance of } \\
\text { getting a position. However, this is pretty meaningless given it is based on statistics of } \\
\text { what did happen, not necessarily what will happen in a similar situation. The "chance" } \\
\text { is probably based on a number of factors we don't know about. }\end{array}$ \\
\hline 34 & Men who fail the psychometric tests have a $30 \%$ chance of gaining a position. \\
\hline 38 & That passing a psychometric test ensures a $70 \%$ chance of receiving a position. \\
\hline 44 & $\begin{array}{l}\text { Even if the applicant has failed the psychometric test, they still have a } 30 \% \text { chance of } \\
\text { getting the job. }\end{array}$ \\
\hline 53 & $\begin{array}{l}\text { If the individual fails the psychometric test, he will still have a } 30 \% \text { chance of getting a } \\
\text { position. }\end{array}$ \\
\hline 58 & $\begin{array}{l}\text { There is a } 30 \% \text { chance that an applicant will get an position even though his result of } \\
\text { a psychometric test did not reach pass cut. }\end{array}$ \\
\hline 74 & $\begin{array}{l}\text { If an applicant fails the psychometric tests, they still have } 30 \% \text { chance to get a } \\
\text { position. }\end{array}$ \\
\hline 75 & Those who fail have a $30 \%$ chance of getting the job. \\
\hline
\end{tabular}

\section{Other chance interpretations}

16 An applicant has a greater chance of getting a position if he passes the psychometric tests.

21 If a person fails a psychometric test when going for a job he will have a much smaller chance of getting that job than a person who has passed the test.

Meaning just because an applicant fails the psychometric test, there is still a chance he will still be hired, probably on other factors.

The probability of an applicant who has failed the psychometric test still getting a position is 0.3 .

\section{Natural ambiguous interpretations}

28 There's a chance that 30 people in 100 people who fail the tests will get the position applied for.

35 The applicant will fail to get a position 7 times out of 10 applications.

76 Assuming the same for a he or she then the applicant has three out of ten chances of getting the job out of those that failed the test.

\section{Relative ambiguous interpretations}

I5 Candidates who fail the tests are $70 \%$ likely to not get a position

Table continues 


\begin{tabular}{|c|c|}
\hline Participant & Statements \\
\hline \multicolumn{2}{|r|}{ Other ambiguous interpretations } \\
\hline | | & The proportion of psychometric test failures who will successfully gain a position. \\
\hline 14 & The decision to take on a person is not solely based on a psychometric test \\
\hline 25 & $\begin{array}{l}\text { A person's potential employability is not governed solely on the basis of passing a } \\
\text { psychometric test, for though those that pass this test stand in a significantly better } \\
\text { position to obtaining employment, those that fail still stand a chance of winning a } \\
\text { position. Explanation of these circumstances is seen by realising that though results } \\
\text { play a large, determining role, it is not the only factor considered by the employers. }\end{array}$ \\
\hline 30 & $\begin{array}{l}\text { Despite failing the psychometric tests, there is roughly a one third chance of still } \\
\text { getting a job }\end{array}$ \\
\hline 50 & $\begin{array}{l}\text { It means that there is no relationship between the chance of passing of psychometric } \\
\text { tests and chance of getting a position. }\end{array}$ \\
\hline 55 & $\begin{array}{l}\text { Successful completion of the psychometric test does not give you a } 100 \% \text { guarantee } \\
\text { of getting a position }\end{array}$ \\
\hline 60 & $\begin{array}{l}\text { To fail the tests does not prevent from getting the position (getting the position will } \\
\text { then be determined by the personality and the motivation of that applicant. }\end{array}$ \\
\hline 78 & $\begin{array}{l}\text { an applicant is less likely to get a position following failure of the tests than following } \\
\text { a pass. }\end{array}$ \\
\hline 79 & Don't know \\
\hline
\end{tabular}

\section{sk}

